\title{
Population genetics of the school shark (Galeorhinus galeus) in New Zealand, Australian and Chilean waters
}

\author{
By
}

\section{Sebastián Ignacio Hernández Muñoz}

\section{A thesis}

Submitted to Victoria University of Wellington

in fulfillment of the requirements for the degree of

Doctor of Philosophy in Cell \& Molecular BioScience

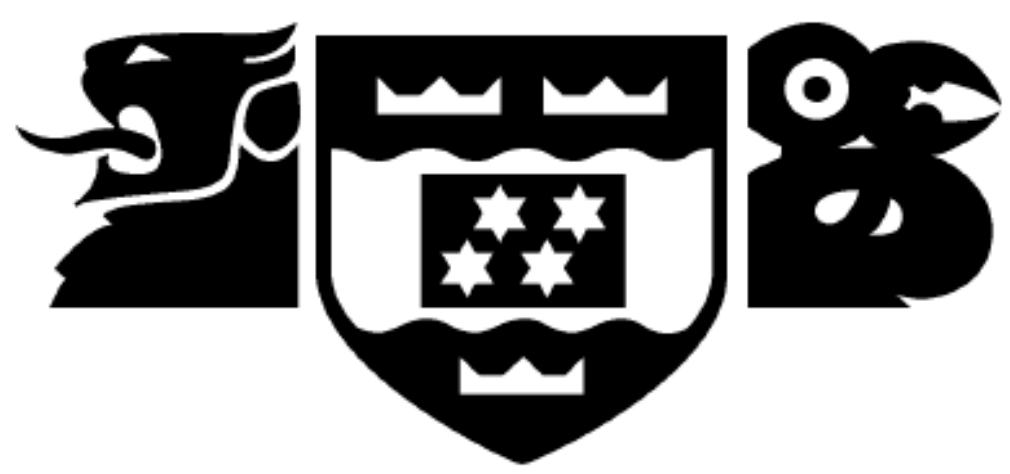

Victoria University of Wellington

Te Whare Wānanga o te Ūpoko o te Ika Māui 

This thesis was conducted under the supervision of:

\author{
Dr. Peter A. Ritchie (Primary Supervisor) \\ Victoria University of Wellington \\ Wellington, New Zealand
}

and

\author{
Dr. Malcolm Francis (Secondary Supervisor) \\ National Institute of Water and Atmospheric Research \\ Wellington, New Zealand
}


This thesis is dedicated to the memory of my grandmother (Tally) 


\begin{abstract}
The school shark (Galeorhinus galeus) is a coastal bentho-pelagic species that is highly migratory and has a widespread distribution in temperate waters. This species matures late, has a relatively low fecundity and is slow growing, which makes it vulnerable to overfishing. They are commercially fished throughout their distribution, and some global stocks have been under pressure because of poor management. In Australia, longline and gillnet fisheries targeted pregnant females and juveniles around Victorian and Tasmanian nursery grounds, resulting in loss of historical inshore nursery habitat. School shark tagging programmes have reported migration between Australian and New Zealand stocks, but preliminary genetic studies have suggested that there are slight genetic differences between the stocks. Currently, the Australian and New Zealand school shark fisheries are assessed and managed as separate stocks. However, the question of whether this species is comprised of a single population or multiple sub-populations in the South Pacific remains unresolved. Given the commercial importance of the school shark fisheries and the concern about stock levels on the regional and trans-Tasman scales, knowledge of stock structure is essential for effective management. The aim of this thesis research was to determine the levels of genetic diversity and population structure of G. galeus in New Zealand and Australia, and compare these to a population in Chile, using mitochondrial DNA (mtDNA) sequencing and microsatellite DNA markers.
\end{abstract}

The DNA sequence of an 893 base pair region of the mtDNA control region (CR) was determined using 475 school shark samples and nine microsatellite DNA loci were genotyped in 239 individuals. Analyses of the data revealed strong evidence of genetic differentiation between G. galeus populations in Australasia and Chile, suggesting restricted gene flow among populations in the western and eastern areas of the Pacific Ocean. The $F_{\mathrm{ST}}$ values ranged from 0.188 to 0.300 for CR mtDNA, and 0.195 to 0.247 for microsatellite DNA in G. galeus. However, there was no evidence of stock differentiation among New Zealand/Australian sample sites for either mtDNA or microsatellite DNA data. These results support the model of a single panmictic stock across the Tasman Sea. The similarity of the results obtained from the maternally inherited mtDNA and biparental inherited microsatellite loci did not support the suggestion of sex-biased dispersal of $G$. galeus in the New 
Zealand/Australia region and it was concluded that females and males had similar patterns of dispersal.

Sharks can be either monogamous or polygamous, which is important when considering stock assessments and harvesting models. Multiple paternity has been reported in several shark species, however, the number of sires per litter varies considerably among species. An investigation of multiple paternity (MP) was conducted in G. galeus by assessing the levels of relatedness within progeny arrays using six polymorphic microsatellite DNA markers. Five "families" (mother and litters) were sampled from the North Island of New Zealand and a parentage analysis was conducted. The minimum number of males contributing to each progeny array was estimated by identifying the putative paternal alleles by allele counting and reconstructing multilocus genotypes method. The analysis showed the occurrence of genetic polyandry in G. galeus; two of five litters showing multiple sires involved in the progeny arrays (40\%). The minimum number of sires per litter ranged from one to four. Although MP was only detected in two litters, this finding is consistent with the known reproductive characteristics of $G$. galeus. It can potentially store sperm for long periods of time and has a specific mating season when males and females typically mix on the edge of the continental shelf. Detecting MP within a litter has highlighted the importance of the post-copulatory selective processes in the G. galeus mating system, and this has implications for the management and conservation of genetic diversity. 


\section{Acknowledgments}

I would like to thank to my supervisor and good friend Dr. Peter Ritchie for giving me the opportunity to work in his lab group and for all his relevant advice and repeated editing ("Spanglish") of my writing and reporting. I am also grateful to my cosupervisor and good friend as well, Dr. Malcolm Francis who provided me with logistical support to get through this thesis, and much advice and editing of this thesis. At the same time, I would like to acknowledge my parents for their constant support and the opportunity to keep studying Marine Science. They have always encouraged us (my brother, Felipe and me) to explore the animal world during our childhood by reading books about the marine and terrestrial world and spending many hours with us on field trips and excursions to zoos/aquariums/museums.

Thanks to all people who supported me during with my fieldwork; during the shark surveys and sampling trips (i.e. fishermen, technicians and people from the National Aquarium in Napier and Kelly Tarltlon's Aquarium in Auckland). I would like to especially thank Clinton Duffy and Ross Daley and their families for their hospitality, and sharing good moments especially those times on my $\mathrm{PhD}$-field trip. They were some of the best moment of my LIFE!!! setting longlines or well-named in Chile as "marrajera", fishing and sampling several species of Chondrichthyes species (sharks, skates, rays, and elephant fish) in New Zealand and Australia waters. I am also grateful to Scott and Sue Tindale for the collection of four pregnant females from Kaipara harbour, and their unconditional help to obtain tissues samples of G. galeus during my $\mathrm{PhD}$; to Frank Webb, Murray Goss, Violet \& Charles Dess for your help as fishing operators to set the longlines and support me with accommodation during, $\mathrm{y}$ field trips.

I would like to thank wife Gelant Vilches for her support and numerous good times spent in New Zealand. I also want to thank to Julieta Astudillo for having the opportunity to know her and enjoying beautiful moment together in New Zealand, especially at School of Biological Science where she became a well-known person. I acknowledge my daughter Amara Hernandez and all my family in Chile whom always supportted and encouraged me to finish my studies.

I have to thank several people from Peter's lab group, especially Sebastien Rioux Paquette, Elizabeth Hegg and Monica Gruber, who have supported me in the lab, help 
me understand the many computer programs and much good advice about population genetic. I also appreciate the good times when I was living in the lab and made so many good mates, many Saludos! and Gracias! to Ange Flemming, Christian Boedeker, Nicky Fitzgibbon, Jessie Prebble, Henry Lane, David Ashton, Jack Xinkang Du.

I would like to thank many good friends Alejandro Perez, Erasmo Macaya, Andrea Varela, Sergio Carrasco, Daniela Diaz, Ursula Rojas y familia, Mauricio Cifuentes y familia, Patricio Quintana, Alexandra Sèbastien, Cristian Canales, Cristian Leaman and Marcela Palominos, Dr. Gaius Wilson, Paul Mardsen, Marry Murray, Alan Hoverd, and the many people from the School of Biological Science whom always supported me in many different ways. Also I am very grateful to Maria GoncalvesRorke and George Allan for giving me good advice and support for my hardship applications for financial support.

I am grateful to CONICYT (Comisión Nacional de Investigación Científica y Tecnológica, Gobierno de Chile) and Victoria University of Wellington (VUW) Scholarships to make it possible for me to study and finish my PhD in New Zealand. I would also like to thank to New Zealand Postgraduate Study Abroad Awards, J. L and Kathleen Stewart Postgraduate Research Experience Travel Award, and the Faculty Strategic Research Grants from Victoria University for financial support. 


\section{Table of Contents}

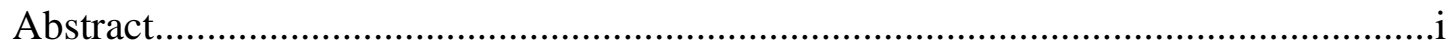

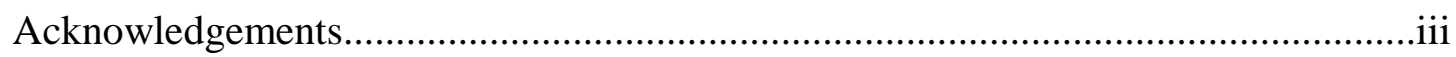

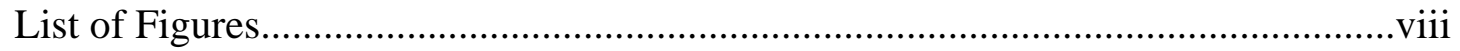

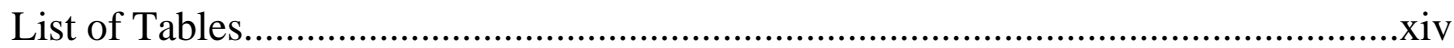

\section{Chapter 1}

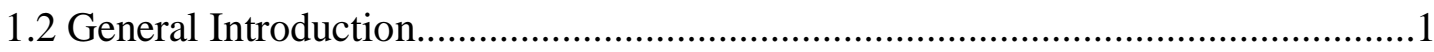

1.2.1 Fisheries management and the stock concept..................................1

1.2.2 Molecular markers for fisheries management.......................................2

1.2.3 Distribution and Biology of Galeorhinus galeus..................................6

1.2.4 New Zealand and Australasian Galeorhinus galeus Fisheries................10

1.2.5 Goals of the thesis research.............................................................13

\section{Chapter 2}

Contrasting the population genetic structures of the school shark (Galeorhinus galeus), northern spiny dogfish (Squalus griffini) and smooth hammerhead (Sphyrna zygaena) using mitochondrial DNA control region sequences

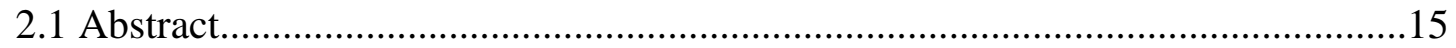

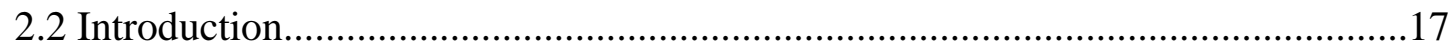

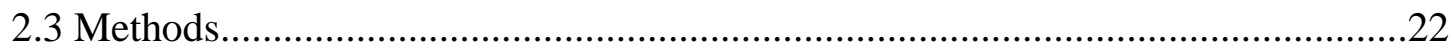

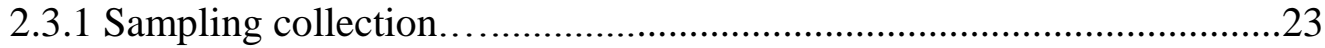

2.3.2 DNA Extraction, amplification and sequencing .................................28

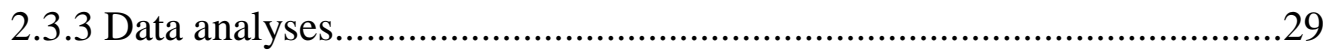

2.3.3.1 Genetic diversity and population structure............................29

2.3.3.2 Mutation-drift and migration-drift equilibrium.......................31

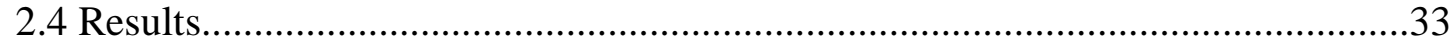

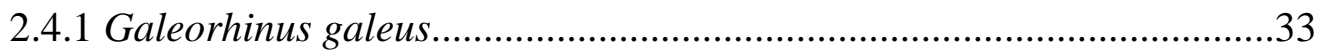

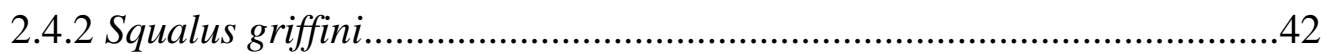

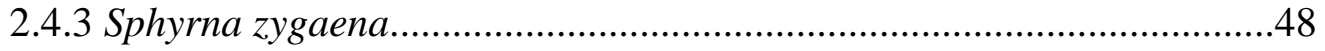

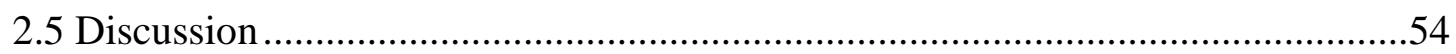

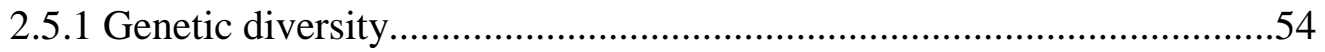


2.5.2 Genetic differentiation and population structure.............................55

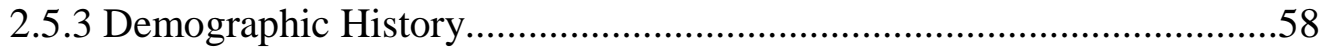

2.5.4 Management implication...........................................................60

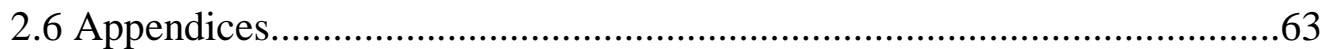

\section{Chapter 3}

The development of microsatellite DNA markers for school shark (Galeorhinus galeus)

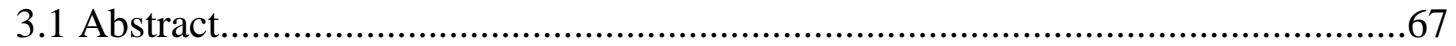

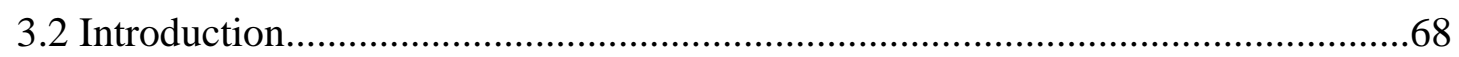

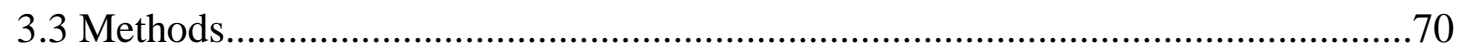

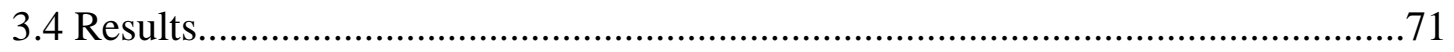

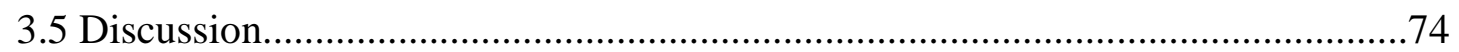

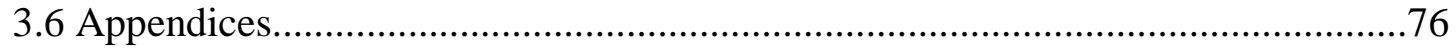

\section{Chapter 4}

Population structure of the school shark (Galeorhinus galeus) in New Zealand, Australian and Chilean waters based on microsatellite DNA markers

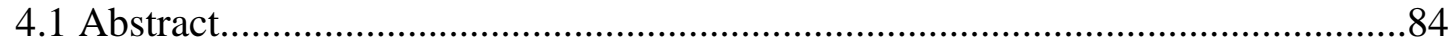

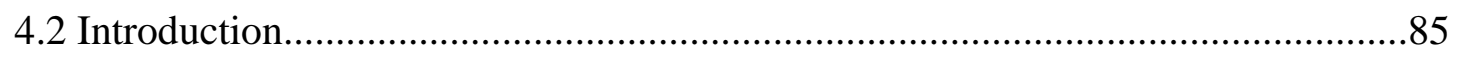

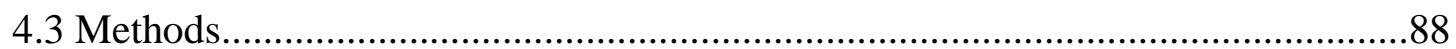

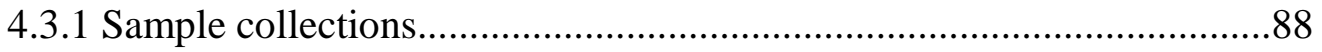

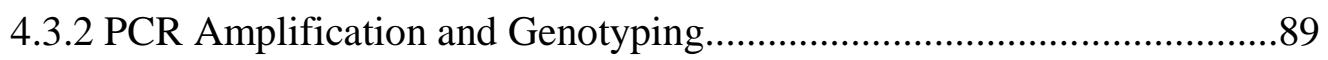

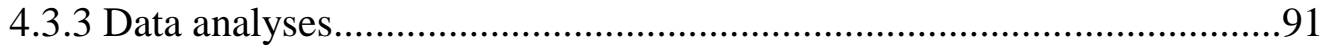

4.3.3.1 Genetic diversity.......................................................... 91

4.3.3.2 Population structure........................................................92

4.3.3.3 Relationship among populations and Bayesian clustering......93

4.3.3.4 Mutation-drift and migration-drift equilibrium.....................94

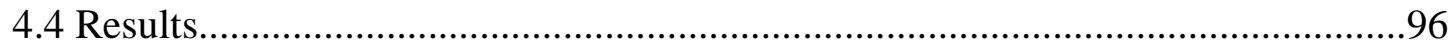

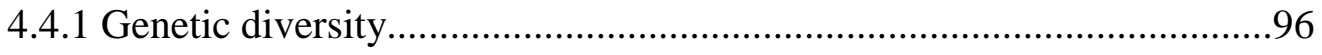

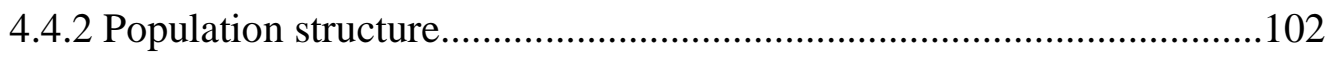

4.4.3 Relationship among populations and Bayesian clustering...................104 


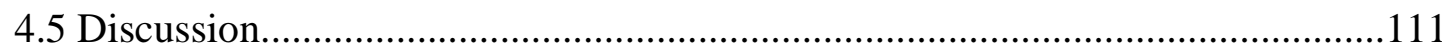

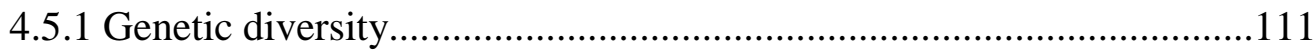

4.5.2 Genetic differentiation and population structure................................112

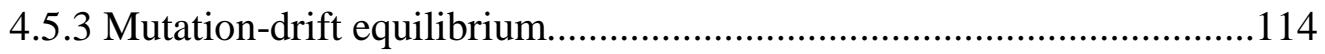

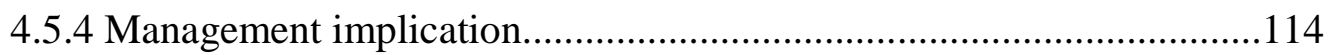

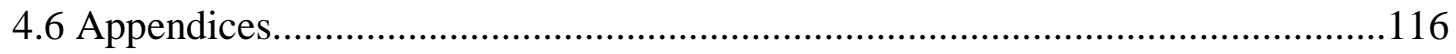

\section{Chapter 5}

Paternity assessment in school shark Galeorhinus galeus in New Zealand waters

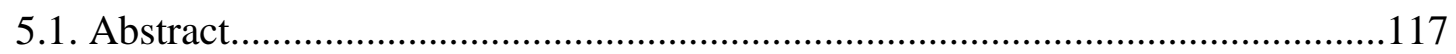

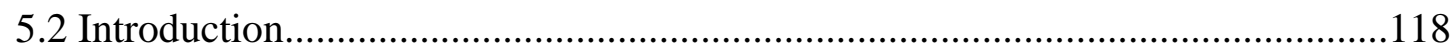

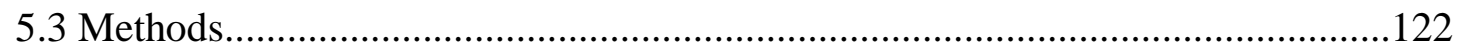

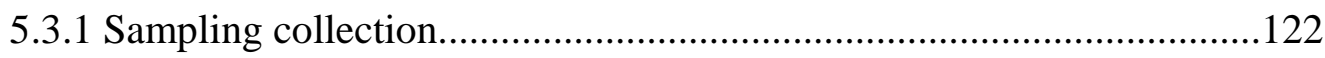

5.3.2 DNA Extraction and Genotyping.......................................................123

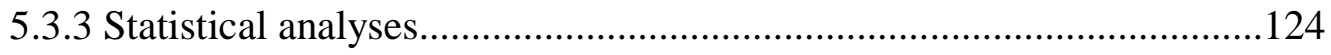

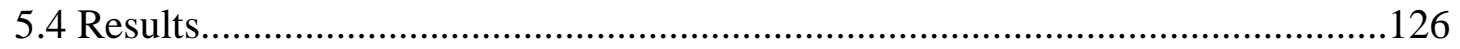

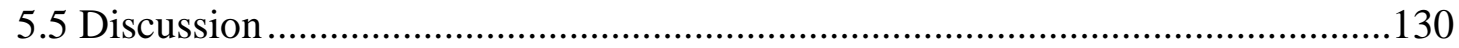

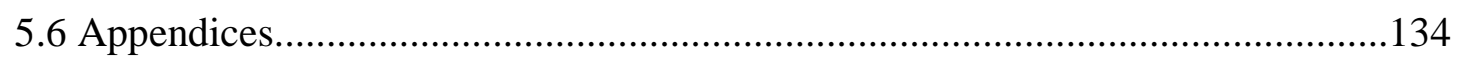

\section{Chapter 6}

General Discussion

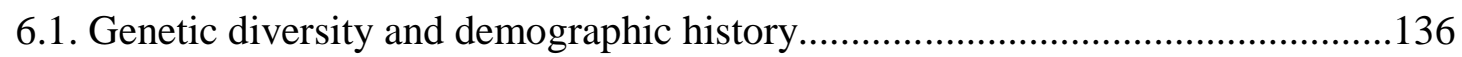

6.2. Genetic differentiation and population structure...............................................142

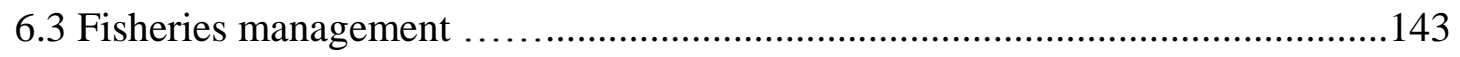

6.4 Recommendation for Galeorhinus galeus management ...............................146

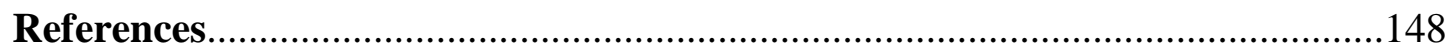




\section{List of Figures}

Fig. 1.1 Movements of New Zealand tagged school shark, Galeorhinus galeus to Australia adapted from Hurst et al. 1999.

Fig. 1.2 Movements of Australian school shark, Galeorhinus galeus within Australian waters adapted from Olsen 1984.

Fig. 2.1 Sampling sites, sample sizes (in parentheses), and geographic distribution (shaded area) of G. galeus collected from New Zealand (NZ), Australia (AUS), and Chile (CHI). A. and B. Sites where adult individuals were sampled. The locations are: ENI (east North Island, NZ), CHA (Chatham Rise, NZ), KAI (Kaikoura, NZ), SOL (Solander Island, NZ), SHO (Shouder, AUS), GAB (Great Australian Bight, AUS), EYR (Eyre, AUS), KIN (King, AUS), TAS (west Tasmania, AUS), FRE (Frederich Bay, AUS), and SAN (Santiago, CHI). The SHO and GAB (SOU), and EYR and KIN (POR) were considered as one location in the analyses (see text details). C. Sites where pups were sampled across the Australasian region. The locations are: KAH (Kaipara Harbour), MAN (Manukau Harbour), NAP (Napier), KAP (Kapiti Coast), MAP (Mapua), GOL (Golden Bay), UPW (Upper Pittwater), STA (St. Helens), and SOR (Port Sorell). D. Individuals collected from Tasmanian waters during 19931994. The locations were: Upper Pittwater (UPW), Blackman Bay (BLA), Norfolk (NOR), Ralph Bay (RAL), Isthmus Bay (ITH), and Swansea (SWA). These locations, excluding SWA, were pooled as HOB (Hobart area) for descriptive genetic diversity, haplotypes distribution, and temporal AMOVA 25

Fig. 2.2 Sampling sites, sample sizes (in parentheses), and geographic distribution (shaded area) of S. griffini collected from New Zealand in GIB (Gisborne), WES (Westland), CHA (Chatham Rise), and KER (Kermadec

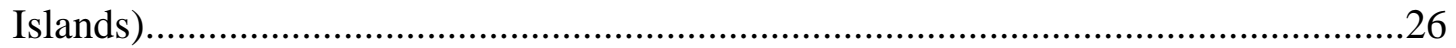

Fig. 2.3 Sampling sites, sample sizes (in parentheses), and geographic distribution (shaded area) of S. zygaena collected from AUS (Australia), NZ (New Zealand), CHI (Chile), and ECU (Ecuador)

Fig. 2.4 Haplotype rarefaction curve for the G. galeus $\mathrm{CR}$ sequences for each population sampled for the open waters sampling. Solid gray lines represent each population in A.New Zealand (ENI, KAI, CHA, SOL), B. Australia (SOU, POR, TAS, and FRE) and C. Chile. Dashed lines represent the 95\% confidence intervals for rarefaction curves.

Fig. 2.5 A. Haplotype genealogy for Galeorhinus galeus from maximum likelihood (ML) tree of $475 \mathrm{CR}$ sequences. Circles represent the haplotypes with area being equivalent to frequency. The number of individuals is reflected inside the circles. Each line indicates one mutational step between haplotypes and small black circles indicates hypothetical missing haplotypes. B. Haplotype frequencies for the adult collection in New Zealand and Australia. The pie charts represent 10 different haplotypes (Hap1-10) and the black pie area reflects 11 private haplotypes pooled as one category called "pri-Hap". The number of private haplotypes by location is detailed in Table 2.1. 
Fig. 2.6 A. Comparison between observed and expected mismatch distributions of pairwise differences sequences of G. galeus under a constant and growth-decline population model performed from adult collection. Solid lines represent the observed pairwise differences, dashed lines the expected distribution for constant population model and dotted lines for growth-decline model. Blue areas encompass $95 \%$ C.I. for observed data. B. Table of neutrality tests, mismatch distribution, co-ancestry coefficient and effective population sizes estimates from CR sequences for three regional populations. The $p$ values are indicated between brackets for the statistics. Tajima test $(D)$, Fu's test $\left(F_{s}\right)$, Harpending's raggedness index $\left(H_{r}\right)$, sum of squared distribution $(S S D)$, population size before expansion $\left(\theta_{0}\right)$, population size after expansion $\left(\theta_{1}\right)$, age of population mutational time $(\tau)$, time since population expansion occurred $(t)$ for mutation rates of $4 \times 10^{-9}$ and $2.15 \times 10^{-9}$, respectively; coancestry coefficient $\left(\theta_{s}\right)$, and effective female population sizes for both mutation rates as well $\left(N_{e f}\right)$

Fig. 2.7 Bayesian skyline plots (BSPs) of G. galeus sequences represent population size changes through time (years) for NZ and AUS populations and CHI separated, inferred with $\mathrm{CR}$ lineages and assuming mutation rates of $4 \times 10^{-9}$ substitution/sites/year (Figure $\mathbf{A}$ and $\mathbf{C}$ ) and $2.15 \times 10^{-9}$ substitution/sites/year (Figure $\mathbf{B}$ and D). Blue areas encompass $95 \%$ highest posterior density (HPD)

Fig. 2.8 Haplotype rarefaction curve for the $S$. griffini CR sequences for each population sampled (GIB, WES, CHA, and KER). Dashed lines represent the 95\% confidence intervals for rarefaction curves..................................................................43

Fig. 2.9 Population structure results for S. griffini from four sites: GIB, WES, CHA, and KER. A. Table of AMOVA results among two groups: first group was composed of GIB, WES, and CHA; and second was KER. The $p$ values are indicated between brackets for the statistics. The d.f. is the degrees of freedom, SSD is the sum of squares, $\mathrm{VC}$ is variance components, and \% of total variation. B. Table of pairwise $\Phi_{S T}$ values (below diagonal) and $p$ values (above diagonal) between populations. The asterisks $(* *)$ represent significant values at $p<0.001$.C. Haplotype genealogy for $175 \mathrm{CR}$ sequences. Circles represent the haplotypes with area being equivalent to frequency. The number of individuals is reflected inside the circles. Each line indicates one mutational step between haplotypes and small black circles indicates hypothetical missing haplotype. D. Haplotype frequencies of CR sequences where the pie charts represent 3 different haplotypes (Hap1, 2 and 5) and the green pie area reflects 11 private haplotypes pooled as one category called "pri-Hap"

Fig. 2.10 Comparison between observed and expected mismatch distributions of pairwise differences sequences of $S$. griffini under a constant and growth-decline population model. Solid lines represent the observed pairwise differences, dashed lines the expected distribution for constant population model and dotted lines for growth-decline model. Blue areas encompass 95\% C.I. for observed data. B. Table of neutrality tests, mismatch distribution, co-ancestry coefficient and effective population sizes estimates from CR sequences for two regional populations: GIBWES-CHA and KER. The $p$ values are indicated between brackets for the statistics. Tajima test $(D)$, Fu's test $\left(F_{s}\right)$, Harpending's raggedness index $\left(H_{r}\right)$, sum of squared 
distribution $(S S D)$, population size before expansion $\left(\theta_{0}\right)$, population size after expansion $\left(\theta_{1}\right)$, age of population mutational time $(\tau)$, time since population expansion occurred $(t)$ for mutation rates of $4 \times 10^{-9}$ and $2.15 \times 10^{-9}$, respectively; coancestry coefficient $\left(\theta_{s}\right)$, and effective female population sizes for both mutation rates as well $\left(N_{e f}\right)$.

Fig. 2.11 Bayesian skyline plots (BSPs) of S. griffini sequences represent population size changes through time (years) for three pooled populations (GIB-WES-CHA) and KER inferred with $\mathrm{CR}$ lineages and assuming mutation rates of $4 \times 10^{-9}$ substitution/sites/year (Figure A and C) and $2.15 \times 10^{-9}$ substitution/sites/year (Figure $\mathbf{B}$ and D). Blue areas encompass $95 \%$ highest posterior density (HPD)................................................................47

Fig. 2.12 Haplotype rarefaction curve for the $S$. zygaena $\mathrm{CR}$ sequences for each population sampled for NZ, AUS, CHI and ECU. Dashed lines represent the 95\% confidence intervals for rarefaction

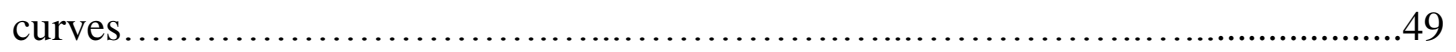

Fig. 2.13 Population structure results for S. zygaena from four sites: NZ, AUS, CHI, and ECU. A. Table of AMOVA results among two groups: first group was composed of NZ and AUS, the second for CHI and ECU.The d.f. is the degrees of freedom, SSD is the sum of squares, $\mathrm{VC}$ is variance components, and \% of total variation. B. Table pairwise $\Phi_{S T}$ values (below diagonal) and $p$ values (above diagonal) between populations. The asterisks $(* *)$ represent significant values at $p<0.001$. C. Haplotype genealogy for $156 \mathrm{CR}$ sequences. Circles represent the haplotypes with area being equivalent to frequency. The number of individuals is reflected inside the circles. Each line indicates one mutational step between haplotypes and small black circles indicates hypothetical missing haplotype. D. Haplotype frequencies of CR sequences where the pie charts represent different haplotypes (Hap1, 2 and 4) and the green pie chart reflect 2 private haplotypes pooled as one category called "pri-Hap"

Fig. 2.14 A. Comparison between observed and expected mismatch distributions of pairwise differences sequences of $S$. zygaena under a constant and growth-decline population. Solid lines represent the observed pairwise differences, dashes lines the expected distribution at constant population model and dot lines at growth-decline model. Blue areas encompass 95\% C.I. for growth-decline model. B. Table of neutrality tests, mismatch distribution, co-ancestry coefficient and effective population sizes estimates from CR sequences for CHI-ECU and Entire dataset. The $p$ values are indicated between brackets for the statistics. Tajima test $(D)$, Fu's test $\left(F_{s}\right)$, Harpending's raggedness index $\left(H_{r}\right)$, sum of squared distribution $(S S D)$, population size before expansion $\left(\theta_{0}\right)$, population size after expansion $\left(\theta_{1}\right)$, age of population mutational time $(\tau)$, time since population expansion occur $(t)$ for mutation rates of 4 x $10^{-9}$ and $2.15 \times 10^{-9}$, respectively; coancestry coefficient $\left(\theta_{s}\right)$, and effective female population sizes for both mutation rates as well $\left(N_{e f}\right)$

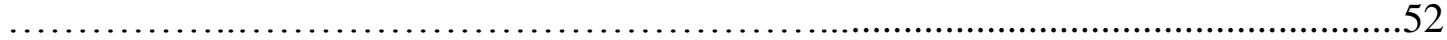

Fig. 2.15 Bayesian skyline plots (BSPs) of S. zygaena sequences represent population size changes through time (years) for to pooled populations (CHI-ECU) populations inferred with CR lineages and assuming mutation rates for mutation rates of $4 \times 10-9$ 
substitution/sites/year (Figure A) and 2.15 x 10-9 substitution/sites/year (Figure B). Blue areas encompass $95 \%$ highest posterior density (HPD) ........................53

Supplementary Fig. 2.1 Relationship between the haplotypes $(h)$ and nucleotide diversity $(\pi)$ of the CR sequences for 21 species of sharks. Only single values were coloured by species, however those species showing similar values were represented in black circles.

Supplementary Fig. 2.2 Haplotype genealogy from maximum likelihood (ML) tree of samples from present study (NZ, AUS, CHI) and a haplotype network adapted according to the one reported by Chabot and Allen (2009) for CR sequences from Peru (PER) and Argentina (ARG). Circles represent the haplotypes with area being equivalent to frequency. The number of individuals is reflected inside the circles. Each line indicates one mutational step between haplotypes. Haplotypes from PER and ARG were obtained from GenBank. Refer to Figure 2.4A for details of NZ/AUS haplotypes geneology....

Fig. 3.1 Distribution of sequence length of 46,602 DNA readings obtained from NGS (Roche 454) . .72

Supplementary Fig. 3.1 Distribution of di-nucleotide, tri-, tetra-, and hexa-, repeats units identified in microsatellite population structure studies reported to the date for 15 shark species. The number in brackets is the number of microsatellite repeat for each motif. The $x$-axis is the number of repeat units for each repeat motif and the $y$ axis is the number of microsatellites identified. 83

Fig. 4.1 Sampling sites, sample sizes (in parentheses), and geographic distribution (shaded area) of adult sharks of G. galeus collected from A. New Zealand, Australia, and B. Chile. The sites are: ENI (east of North Island), CHA (Chatham Rise), SOL (Solander Island), SHO (Shoulder), TAS (West Tasmania), FRE (Frederich Bay), and SAN (Santiago) .88

Fig. 4.2 Allelic frequency distribution for the microsatellite loci Ggal14, Ggal15, and $G g 20$ across all populations of G. galeus. Allele sizes are given in base pairs of PCR products. The letter " $p$ " represents private alleles found in one population. .98

Fig. 4.3 Allelic frequency distribution for the microsatellite loci $G g 15, G g 22$, and Gg23 across all populations of G. galeus. Allele sizes are given in base pairs of PCR products. The letter " $p$ " represents private alleles found in one population. .99

Fig. 4.4 Allelic frequency distribution for the microsatellite loci $G g 12, G g 07$, and Gg11 across all populations of G. galeus. Allele sizes are given in base pairs of PCR products. The letter " $p$ " represents private alleles found in one population. 100

Fig. 4.5 Allele discovery curves for the most polymorphic loci. A. Gg15 (NZ and AUS) and Ggal15 (CHI) and B. Gg12 (NZ and AUS) and $G g 23$ (CHI) 101

Fig. 4.6 2D Plot of the first two principal components axes (PCA) of genotype frequencies for eight microsatellite loci (excluding $\mathrm{Gg} 14$ ) from ENI (east-North Island), CHA (Chatham Rise), SOL (Solander Island) in New Zealand (black circles); SHO (Shoulder), TAS (West Tasmania), and FRE (Frederich Bay) in Australia (gray circles), and SAN (Santiago) in Chile. Figure A represents all collection sites and B all sites excluding SAN. 105 
Fig. 4.7 3D Plot of the analyses of principal components (AFC) of genotype frequencies for eight microsatellite loci (excluding Gg14). Circles represent each individual and different colours are the populations analyzed: ENI (east-North Island), CHA (Chatham Rise), SOL (Solander Island) in New Zealand; SHO (Shoulder), TAS (West Tasmania), and FRE (Frederich Bay) in Australia; and SAN (Santiago) in Chile. 106

Fig. 4.8 Radial neighbor-joining dendrogram for eight microsatellite loci (excluding Gg14) screened from seven populations of G. galeus based on the Nei et al.'s, (1983) $D a$. Numbers are the bootstrap support indices of loci.

Fig. 4.9 Bar plot of Bayesian clustering analysis from $G$. galeus for eight microsatellite loci (excluding Gg14) (239 individuals) performed using STRUCTURE. Each bar represents proportional probability of assignment to each genetic cluster. Sampling sites were organized by region: New Zealand (ENI, CHA, SOL), Australia (SHO, TAS, FRE), and Chile (SAN). A. Admixture and allele frequencies correlated model for $\mathrm{K}=3$. B. Non-admixture and allele frequencies correlated for $\mathrm{K}=3$.). Black, grey, and white components of each bar represent the proportion in each of the three clusters 107

Fig. 4.10 Identification of the number of genetic clusters $(K)$ of best fit observed for eight microsatellite loci under "delta $K$ " $(\Delta K)$ and "log probability of data" (Mean of $\mathrm{LnP} \pm 1$ ) approaches. A. Admixture and allele frequencies correlated model for $\mathrm{K}$ ranging from 1-7. B. Non-admixture and allele frequencies correlated for $K$ ranging from1-7

Fig. 4.11 Distribution of allelic frequencies sizes (X-axis) and the proportion of alleles (Y-axis) for nine microsatellite loci for each population. Black bars represent the proportion of alleles observed in each allele frequency classes.

Fig. 5.1 A. Collection location of G. galeus mother-litters groups (solid circles) of two putative breeding grounds on Kaipara (KP) and Manukau (MK) harbours, and east of North Island (ENI) samples used for population genetic analysis in this study. Numbers in brackets are the sample sizes. B. Table represents G. galeus gravid females (named as Mum) analysed in this study. Total length (TL) is showed in $\mathrm{cm}$ and LS represents litter size.....

Supplementary Fig. 5.1 Plot of the five progeny analysed based on the mean embryo total length (TL) of embryos collected from pregnant females....................................................................... 135

Supplementary Fig. 5.2 Smears of nidamental gland of G. galeus no gravid with immature ovary. Both figures are the same but they are pointed different spermatozoa. The white dash line represents the orientation of each spermatozoa, and arrows indicate the head and tail of the spermatozoa..................................135

Fig. 6.1 Relationship between the haplotypes $(h)$ and nucleotide diversity $(\pi)$ surveyed using RFLP-mtDNA, $C O I, C y t B$ and $C R$ of several population of teleost marine species (black circles) reported in Grant \& Waples 2000 and Varela 2012, and for population the CR sequences for 23 shark species (grey circles). The shark species labelled represents a particular population with high sample size $(>30)$ 140 
Fig. 6.2 Relationship between the expected heterozygosity averaged and number of alleles surveyed using microsatellite loci of Teleost marine species (black circles) reported in DeWoody \& Avise 2000 and Varela 2012, and for 16 elasmobranch species (grey circles). The values of number of alleles and expected heterozygosity were obtained from Table 4.2 in Supplementary Material. The dash line showed the expected relationship of heterozygosity to the effective number of alleles reported in Figure 2 according to Crow \& Kimura (1970) in DeWoody \& Avise 2000. 141 


\section{List of Tables}

Table 2.1 Primer sequences and PCR conditions of CR for each shark species studied.

Table 2.2 Genetic variability from the control region sequences of Galeorhinus galeus for the open waters (OW) and pupping areas (PA). Sampling size $(N)$, number of segregation sites $(k)$, number of haplotypes $(n)$, private haplotypes $(p h)$, haplotype diversity $(h)$, and nucleotide diversity $(\pi)$...

Table 2.3 A. AMOVA among five temporal groups from Australasia region (NZ and AUS), among three spatial groups (NZ, AUS, and CHI), and two spatial groups (NZ and AUS).The d.f. is the degrees of freedom, SSD is the sum of squares, VC is variance components, and \% of total variation. B. Pairwise $\Phi_{S T}$ values (below diagonal) and $p$ values (above diagonal) between NZ, AUS and CHI populations from adult collections. The $p$ values are indicated between brackets for AMOVA, and significant $p$ values for the pairwise $\Phi_{S T}$ are represented by $*(\alpha=0.05)$, ** after sequential Bonferroni correction $(\alpha=0.0014)$. C. Pairwise $\Phi_{S T}$ values between NZ and AUS populations from pup collections. Significant $p$ values are represented by $*$ $(\alpha=0.05)$

Table 2.4 Genetic variability from the CR sequences for $S$. griffini. Sample size $(N)$, number of segregation sites $(k)$, number of haplotypes $(n)$, private haplotype $(p h)$,

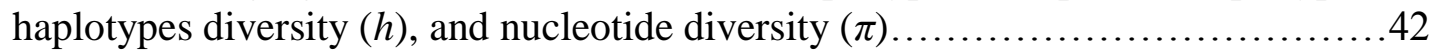

Table 2.5: Genetic variability from the CR sequences for $S$. zygaena. The sampling size $(N)$, number of segregation sites $(k)$, number of haplotypes $(n)$, private haplotypes

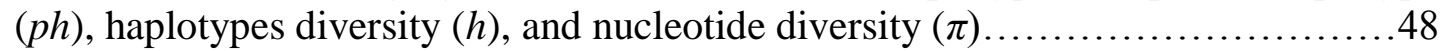

Supplementary Table 2.1 Genetic diversity from the control region sequences among 20 shark species by each sampling locations reported to the date. Sampling locations, sampling size $(N)$, haplotypes diversity $(h)$, nucleotide diversity $(\pi)$, effective female population sizes $\left(N_{e f}\right)$ are detailed by each reference. ID is the reference number for each population in the supplementary figure.

Table 3.1 Characteristics of 15 microsatellite loci for G. galeus. $N=$ sample size screened for each, $\mathrm{A}=$ number of alleles, $H_{\mathrm{o}}=$ observed heterozygosity, $H_{\mathrm{e}}=$ expected heterozygosity, and $F_{\text {IS }}=$ inbreeding coefficient (negative values $=$ heterozygote excess and positive values = heterozygote deficiency). NA means not-applicable.

Supplementary Table 3.1 List of microsatellite loci reported in primer notes to date for elasmobranch species. Three methodology approaches were utilised to develop SSRs: hybridization selection (HS), linker and ligation procedures (LL), expressed sequence tags (ESTs), and Roche 454. The number of loci, number of repeat, observed heterozygosity $\left(H_{\mathrm{o}}\right)$, and expected heterozygosity $\left(H_{\mathrm{e}}\right)$ are reported. Number of repeats and alleles, and observed and expected heterozygosity are represented by ranges across loci. .76

Supplementary Table 3.2 Genetic diversity from microsatellite loci reported to date for population genetic studies among 15 shark species by location. Sampling size $(N)$, number of alleles $(\mathrm{A})$, observed heterozygosity $\left(H_{\mathrm{o}}\right)$, and expected heterozygosity $\left(H_{\mathrm{e}}\right)$ are reported. For Negaprion brevirostris and $N$. acutensis the allele richness i.e. 
asterisk "*" is represented rather than number of alleles due they were not reported by each loci in Schultz et al. 2008. For Squalus acanthias the number of alleles is represented across eight loci, for this reason is only one value.

Table 4.1 Primer sequences and PCR conditions of nine microsatellite loci of $G$.

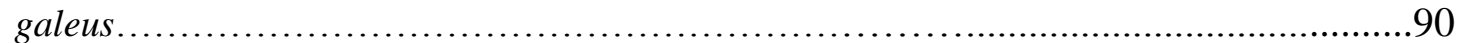

Table 4.2 Summary of nine microsatellite loci for each G. galeus population. Population in New Zealand (NZ), Australia (AUS) and Chile (CHI) are as follows: ENI = east of North Island, $\mathrm{CHA}=$ Chatham Rise, $\mathrm{SOL}=$ Solander Islands, $\mathrm{SHO}=$ Shoulder, TAS $=$ West Tasmania, FRE $=$ Frederich Bay, and SAN $=$ Santiago. Parameters are as follows: $n$, sample sizes; $R_{S}$, allelic richness, $H_{\mathrm{o}}$, observed microsatellite heterozygosity; $H_{\mathrm{e}}$, expected microsatellite heterozygosity; $F_{\text {is }}$, microsatellite inbreeding coefficient [negative values = heterozygote excess and positive values = heterozygote deficiency; $\mathrm{AAL}=$ Average across loci; values marked with $*$ deviate from Hardy-Weinberg expectations nominally after sequential Bonferroni correction (alpha $=0.05 / 63=0.0008)$.

Table 4.3 A. AMOVA results for global panmixia, across ocean basins (New Zealand, Australia, and Chile) based on eight microsatellite loci. The asterisk (*) represents significant values at $P<0.05$, and ** significant values at $P<0.001$. The SSD is the sum of squares, VC is variance components, and $\% V$ is the percentage of total variation. B. Below the diagonal are the pairwise $F_{\mathrm{ST}}$ values (Weir \& Cockerham 1984), in parentheses the $R_{\mathrm{ST}}$ values (Slatkin 1995), and in italics the $D_{\mathrm{ST}}$ values (Jost 2008) for eight microsatellite loci (excluding Ggal14). Above the diagonal is the number of loci, name of loci (superscript), and $P$ values for Exact $G$ tests from nine microsatellite loci (including Ggal14) between New Zealand, Australia and Chile populations. Significant $P$ values for the pairwise $F_{\mathrm{ST}}$ and Exact tests are represented by $* P<0.001$. 103

Table 4.4 Results from the BOTTLENECK tests and $M$-RATIO analysis of eight microsatellite loci (excluding $G g 14$ ) for each population. Significant $P$ values for Wilcoxon's test are represented with the asterisks..

Supplementary Table 4.1 A. Genetic diversity at microsatellite loci reported to date for population genetic studies among 16 Elasmobranch species. Number of populations studied ( $N$ pop), number of loci ( $N$ loci), number of individuls screened across loci $(N$ ind), number of alleles averaged across all loci $(A)$, and expected heterozygosity averaged across all loci $\left(H_{\mathrm{e}}\right)$ are reported. Note for Carcharodon carcharias only observed heterozygosity $\left(H_{\mathrm{o}}\right)$ was reported in Pardini et al. (2001). B. Genetic diversity at microsatellite loci in teleost freshwater, teleost anadromous, teleost marine, and elasmobranch species. C. Graphical representation of Table B for four different groups of species. 116

Table 5.1 Allelic frequency distribution and genetic diversities at six microsatellite loci for 45 G. galeus sharks from New Zealand waters i.e. East of North Island (ENI) and mums collected in the present study. Number of sharks scored $\left(N_{\mathrm{i}}\right)$, Number of alleles (A), observed heterozygosity $\left(H_{\mathrm{o}}\right)$, expected heterozygosity $\left(H_{\mathrm{e}}\right)$, and the probabilities of Hardy Weinberg Equilibrium $\left(P_{\mathrm{HWE}}\right)$. The asterisk $(*)$ means deviate from Hardy-Weinberg expectations nominally after sequential Bonferroni correction (alpha $=0.05 / 6=0.008)$. Exclusion probabilities $(E p)$ estimated in GERUD is

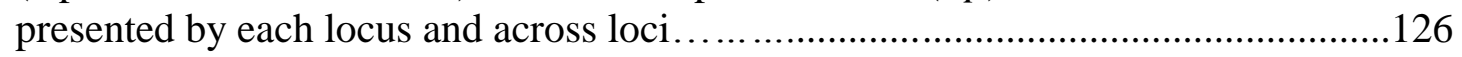


Table 5.2 Probability of detecting MP (PRDM) values for six microsatellite loci used assuming six mating schemes and specific litter size for each Family and overall families.

Table 5.3 Galeorhinus galeus Families (Mum $=$ mother and Emb $=$ embryo) genotypes consistent with MP.

Table 5.4 Summary of analysed Families (mums and offspring) of G. galeus genotypes consistent with MP inferred in GERUD2.0. The number of sires (\# sires) were estimated by allele counting (AC) using a multilocus genotypes (see details Table 5.3), and GERUD. The number of solution (\# solutions), number of progeny (\# progeny) and the probability score $(P S)$ were obtained from GERUD as well.

Supplementary Table 5.1 Summarize of the genetic mating systems studies reported to the date in elasmobranch species classified by reproductive strategy (RS), which are indicated as oviparity $(\mathrm{O})$, aplacental viviparity (AV), and placental viviparity (PV). The number of microsatellite loci (No. of msat), number of litter (No. litter analysed), average number of litter size (Average litter size), number of progeny (No. progeny analysed), range of sires per litter, and percentage of litter with MP (\% litter with MP) are reported for each species. The asterisk indicates the only study that use MHC II $\alpha$ locus instead of microsatellites loci.................................. 134 


\section{Chapter 1}

\subsection{General Introduction}

\subsubsection{Fisheries management and the stock concept}

Fish are the only major human food item that is still harvested from the natural environment. Marine fishes have become of particular concern due to their high level of exploitation and their use in the aquaculture industry (FAO 2012). However, good management is essential to ensure the sustainable use of a natural biological resource in order to maintain the productivity of a stock (Musick \& Bonfil 2005). Crucial to management is an accurate stock assessment because it is typically used to set the regulations and estimate the maximum sustainable yield of a fishery. A stock assessment model is based on a range of fisheries and biological data, including age structure and growth rates, fecundity, sex ratio, life history, mortality, migratory patterns and total biomass. The first step in the process of conducting a stock assessment is to define the harvested or managed unit of a fishery. A stock is most often defined based on geographic location, but natural populations can be spread over a wide range (Walker et al. 2008). It can be difficult to match the most appropriate biological unit of a fishery species to a stock assessment.

A fisheries stock assessment model is developed based upon the notion of an ideal "stock". Several stock definitions have been discussed in Carvalho \& Hauser 1994 and Grant \& Waples 2000. In general "stock" refers to a group of organisms whose demographic and genetic trajectory is largely independent from other such groups (Waples 1998). Several approaches have been used to identify a "stock" and these can be classified as direct or indirect methods. Direct methods of egg and larval surveys, and tagging data (mark/recapture) have provided a useful understanding of the stock movements in many fish species (Kohler \& Turner 2001). However, these methods are often logistically difficult to undertake, involving intensive surveys that yield relatively little data, and are unable to determine whether immigrants to a stock will survive and reproduce (Waples 1998). For this reason, it is considered important to use genetic markers, which provide an indirect estimation of the relative contribution of migration and/or dispersal among stocks (Waples 1998, Selkoe et al. 
2008). When genetic data are combined with other approaches (the direct methods) patterns of genetic connectivity and levels of population structure are more likely to be determined.

The application of the stock concept has been a challenge for stock assessment purposes. This is because the ocean has been viewed as an environment that lacks dispersal barriers (Waples 1998, Ward 2000). However, complex patterns of genetic structure have been reported in many teleost (Ward \& Elliot 2001) and elasmobranch species (Dudgeon et al. 2012). For instance, several coastal species exhibit low levels of gene flow between Australia and New Zealand, which suggests oceanographic and environmental conditions are acting as barriers to gene flow in the Tasman Sea (Ward $\&$ Elliot 2001). In addition, shark species through the eastern Pacific have different dispersal patterns: a) by stepping stones of islands in some populations connected by coastline in the scalloped hammerhead, Sphyrna lewini (Duncan et al. 2006); b) a complete separation is considered for some coastal species such as lemon shark, Negraprion brevirostris and sicklefin lemon shark, N. acutidens (Schultz et al. 2008), Galeorhinus galeus (Chabot \& Allen 2009), and S. zygaena (Testerman et al. 2008) or no evidence of isolation of oceanic and pelagic species such as S. lewini (Duncan et al. 2006) and blacktip shark, Carcharhinus limbatus (Keeney \& Heist 2006). These findings have provided an important understanding of the levels of genetic connectivity in several marine fishes, and emphasize the use of genetic methods for understanding dispersal patterns.

\subsubsection{Molecular markers for fisheries management}

The application of genetic markers to fisheries management has resulted in significant insights into the population ecology of exploited marine species. Genetic studies have demonstrated genetic differentiation among marine populations, over small to large geographic scales (Laikre et al. 2005, Dudgeon et al. 2012). The roles of genetic methods in fisheries science are, a) to determine the number of stocks and the level of genetic connectivity, and b) to determine the level of genetic variation in a stock so it can be managed to preserve its evolutionary resilience (Carvalho \& Hauser 1994, Laikre et al. 2005). The evolutionary processes of mutation, natural selection, random genetic drift and migration influence genetic variation in a population. Genetically 
isolated populations diverge from each other typically because the evolutionary processes of drift and selection operate independently in each group. However, two populations will be genetically similar even if there is a small amount of migration between them (Waples 1998). Genetic connectivity between populations is primarily a function of the number of migrants $\left(m N_{e}\right.$, where $m=$ migration rates and $N_{e}=$ effective population size) and theoretically only one migrant per generation is needed between populations to homogenize genetic variation. In contrast, demographic connectivity between populations is a function of the proportion of migrants $(m)$ exchanged. When there has been no migration between two stocks genetic markers will generally have the power to demonstrate this, suggesting that both stocks are also demographically uncoupled. In contrast, when even small amounts of migration occur between stocks genetic markers will typically show connectivity, but the demographic connectivity between stocks is less certain; they could or could not be demographically coupled.

Recent technological advances in molecular genetics have improved the resolution to understand the genetic connectivity among populations. Several types of molecular markers have been developed and utilized for fisheries management, which can be categorized as either protein-based markers (i.e. allozymes) or DNA-based markers. Allozyme electrophoresis was the first technique used to assess genetic stock structure in elasmobranchs (Smith 1986, MacDonald 1988, Heist et al. 1995, Ward \& Gardner 1997, 1998). Subsequently, DNA techniques became available that used mitochondrial DNA (mtDNA) restriction fragment-length polymorphism (RFLP) (Heist et al. 1996a, b, Ward \& Gardner 1997). For example, genetic analyses of allozymes and RFLP have shown that G. galeus is a single species worldwide and genetically different among ocean basins (Ward \& Gardner 1997). On a smaller geographic scale, these studies showed only weak levels of differentiation between east coast of New Zealand and west Tasmania in Australian populations. However, the lack of genetic variation detected using these molecular markers and limited number of sample collection sites, makes this finding of limited use.

More recently, the polymerase chain reaction (PCR) amplification and direct sequencing of specific genome regions allowed more DNA loci to be sampled, which substantially improved the level of statistical resolution e.g. mtDNA control region and microsatellites (nuclear DNA/nDNA) (Heist 2005, Dudgeon et al. 2012, Portnoy \& Heist 2012). A range of sample DNA qualities could be utilized by PCR-based 
techniques making sample collecting and sharing much easier. Choosing DNA markers that were assumed to be neutral became important because the results were less likely to be confounded by any adaptive processes (selection).

Neutral molecular markers provided insight into the processes of migration (gene flow) and genetic drift. They are called "neutral" based on the neutral theory of evolution proposed by Motoo Kimura (1964). This theory states that polymorphysms of a population are selectively neutral, and their evolutionary fate is determined by genetic drift (random fluctuation of gene frequencies) (Kimura 1989). The rate at which one allele was subtituted in a population $(k)$ would have equal rates at which one mutation arose each generation $(\mu)$. In other words, the level of polymorphism is proportional to the mutation rate that arose each generation in a population. It means that random genetic drift and mutation play a significant role in evolution or in forming the genetic structure of the species (Kimura 1989).

The mtDNA was the first marker to be used in a genetic structure studies. The mtDNA is maternally inherited, and it has an elevated mutation rate. The mtDNAgenes are relatively easy to amplify due to conservative sequence regions (i.e. transfer ribonucleic acids, tRNAs), and a wide range of PCR primers are available. The mtDNA genome has a quarter the effective population size $\left(N_{e}\right)$ when compared to nuclear gene regions, which increases its sensitivity to genetic drift and theoretically it should more often show differentiation between isolated populations (Nielsen et al. 2009). However, all genes on the mtDNA genome are completely linked and this molecule only represents the genetic patterns of a single locus, which limits its statistical power.

One of the most useful mtDNA regions for analysing stock structure in elasmobranchs is the "control region" (CR). It is usually the only non-coding region in the vertebrate mtDNA genome due to its role in replication and transcription of mtDNA molecules. The vertebrate $\mathrm{CR}$ is subdivided in three domains that differ from each other in base composition, rate and mode of evolution. The central domain of CR is relatively conserved, whereas the flanking regions are typically hypervariable (Saunders \& Edwards 2000, Heist 2005). For this reason (fast rate of evolution), the whole $\mathrm{CR}$ is commonly used to determine levels of polymorphism and enable to estimate the current status of population structure of species. Many studies have used 
the CR to assess genetic structure in shark species (Pardini et al. 2001, Keeney et al. 2005, Duncan et al. 2006, Stow et al. 2006, Lewallen et al. 2007, Ahonen et al. 2009, Chabot \& Allen 2009, Dudgeon et al. 2009, Benavides et al. 2011, Karl et al. 2011, 2012). These have revealed different dispersal capabilities among species, which result in different levels of stock structure.

Microsatellites are now one of the most popular markers in population genetic studies. They are co-dominant DNA markers that are inherited in Mendelian terms. They are defined as Simple Sequence Repeat (SSRs) or Short Tandem Repeats (STRs) widely distributed through the chromosomes. Microsatellites consists of motifs of one to six nucleotides repeated several times e.g. $\mathrm{TG}_{10}$, which means an array comprising the sequence TG repeated 10 times (Li et al. 2002, Guichoux et al. 2011). Most microsatellites are dinucleotide repeats and the length of those repetitive arrays varies among taxa. For example, fishes have the longest dinucleotide repeats (>20 repeat unit with a maximum of 87 , Schug et al. 1998), whereas insect species varies considerably (between 16 for social wasp Parachartergus coloboperus and $>30$ for Drosophila melanogaster; Schug et al. 1998).

Despite of mechanisms of develop of microsatellite is not completely understood, it is commonly thought that evolves by replication slippage. This process involves a gain or reduction of one or more repeat unit (Levinson \& Gutman 1987, Schlötterer \& Tautz 1992, Ellegren 2004). For example the (TG) 10 mentioned in the previous paragraph might become and $(\mathrm{TG})_{8}$ or $(\mathrm{TG})_{12}$ in some individual's offspring. Several models have been proposed to explain and predict the mutation processes that affect microsatellite evolution (Estoup et al. 2002). The most adopted model is the stepwise mutation model (SMM). The model states that microsatellites mutations alters the length of a repetitive array though the gain or removal one unit (Kimura \& Ohta 1978). The infinite allele model (IAM) assumes that mutations may create and infinite number of repeat with allelic states not presented in the allelic distribution (Kimura \& Crow 1964). The two-phase model (TPM) is an extension of the SMM and it considers the probability for a microsatellite mutation to involve more than one unit (Di Rienzo et al. 1994).

Mutations cause a change in the overall length of the region containing the microsatellite locus, and consequently the quantification of alleles distinguishable by 
molecular size ( $\mathrm{Li}$ et al. 2002). The primary methodology used for detecting variation of microsatellite allele lengths is amplification of the microsatellite containing-region by PCR and using electrophoresis to determine the sizes of the PCR products. Fluorescent dye labelling technology has enabled microsatellites loci to be combined and analysed together whenever their allele sizes do not overlap, and if they do overlap different coloured fluorescent dyes can be used. This approach expands the scope of the genetic analysis to include multiplexing and pool-plexing different loci together, consequently reducing the cost and time (Guichoux et al. 2011).

Microsatellites markers are used as tools for a wide range of applications. They are applied for molecular forensics, parentage analysis, genetic structure and connectivity, and phylogenetic relationships (Guichoux et al. 2011). To date, several polymorphic loci have been developed in different species of elasmobranchs (Heist \& Gold 1999, Pardini et al. 2000, Feldheim et al. 2001a, 2001b, Schrey \& Heist 2002, Heist et al. 2003, Keeney \& Heist 2003, Chapman et al. 2004, McCauley et al. 2004, Portnoy et al. 2006, DiBattista et al. 2008, Chabot \& Nigenda 2011, Broomer \& Stow 2010). Compared to mtDNA, microsatellites nDNA markers enable different levels of genetic resolution because they sample more independent points (regions) on the genome (Nielsen et al. 2009). In spite of microsatellite loci studies in shark showed high levels of genetic diversity compared with mtDNA studies, little evidence of genetic differentiation has been found i.e. within ocean basins (Dudgeon et al. 2012). However, any attempt to estimate structure of highly mobile marine species, such as sharks, will be challenging for any molecular markers. (Graves 1998, Heist et al. 2005) All these considerations should be taken into account to provide useful information about the stock structure in shark species.

\subsubsection{Distribution and Biology of Galeorhinus galeus}

The school shark (Galeorhinus galeus) belongs to the family Triakidae, and it has a widespread distribution in temperate waters of the eastern North Atlantic, western South Atlantic, north eastern and south eastern Pacific, South Africa, New Zealand and Southern Australia (Last \& Stevens 2009). It occurs all around New Zealand, but its distribution in Australia is restricted to southern waters from Moreton Bay (southern Queensland) to Cape Leeuwin (Western Australia), including Lord Howe 
Island and Tasmania (Last \& Stevens 2009). A semi-pelagic species, it inhabits the continental shelf from nearshore to $660 \mathrm{~m}$ in depth (Anderson et al. 1998, Walker 1999, Hurst et al. 2000, West \& Stevens 2001), and also occurs in the pelagic zone and offshore (Walker 1999, Bagley et al. 2000).

Galeorhinus galeus, as well as many other species of elasmobranchs, exhibit a low reproductive potential. This is characterised by low fecundity, slow growth, late sexual maturity, and high longevity (Olsen 1954, 1959, 1984, Grant et al. 1979, Francis \& Mulligan 1998, Hurst et al. 1999). They are aplacental viviparous and their fecundity varies between 15-52 pups depending on the size and age of the mother (Olsen 1954, 1984, Walker 1999, Walker 2005). Sexual maturity occurs at an older age in females than males, with females reaching maturity at about 13-15+ years (124-140 cm total length, TL), whereas in males it is at about 8-17+ years (125-135 cm TL) (Olsen 1954, 1984, Francis \& Mulligan 1998, Walker 2005). The longevity of New Zealand G. galeus is still uncertain, however the oldest known shark was estimated to be 25 years old (Francis \& Mulligan 1998). By contrast, results from an Australian long-term tag recovery study (after 42 years), have suggested that $G$. galeus could attain an age of $\sim 60$ years (Walker 1999).

The reproduction of $G$. galeus has been well described through its distribution range. The reproductive cycle in female is characterised by a long ovarian and gestation period estimated to be three years (triennial) (Olsen 1984, Peres \& Vooren 1991, Walker 2005). Therefore pregnant females give birth approximately every 3 years (Peres \& Vooren 1991, Walker 2005). This means that G. galeus population will comprise females at three different reproductive stages: (a) about one-third of the population consist of sharks with resting oviducts (after parturition) and vitellogenesis in the ovary; (b) another one-third of the population will have large mature follicles, oviducal gland and uteri ready for ovulation; and (c) one-third will have full-term embryos in the oviducts (Peres \& Vooren 1991, Walker 2005). This fact has biological significance because only one-third of the mature females will be supporting the annual recruitment of newborns.

Tagging data from larger G. galeus have been obtained in Australia and New Zealand waters. These studies have showed national or international migration patterns in and between Australian (Olsen 1953, 1954, Stanley 1988, Walker 1989, 
Walker et al. 1997) and New Zealand waters (Paul 1988, Francis \& Mulligan 1998, Hurst et al. 1999, Francis 2010). One tagged female released in the Great Australian Bight (Australia) was recaptured 3,500 km away, after 4.1 years, between the North and South Islands of New Zealand. It was released again and recaptured off Australia 2,600 km away, 2.8 years later (Walker et al. 2008). The maximum recorded distance travelled by a shark is $4940 \mathrm{~km}$ after 9.6 years (Hurst et al. 1999). The distance travelled increased significantly after the first year of liberty. Thirty percent of $G$. galeus tagged in New Zealand were recaptured outside the release Quota Management Area (QMA) within 1-2 years; after 2-5 years, 60\% were recaptured outside the release QMA, and a significant proportion of sharks emigrated to Australian waters (23\%) (Francis 2010). Due to relatively high emigration rates from New Zealand to Australian waters, Hurst et al. (1999) considered both populations to be the same stock unit. These findings suggest that there is trans-Tasman exchange of migrants, which presumably allows the physical mixing of the G. galeus population at a level that is significant enough to affect the stock structure (Figure 1.1).

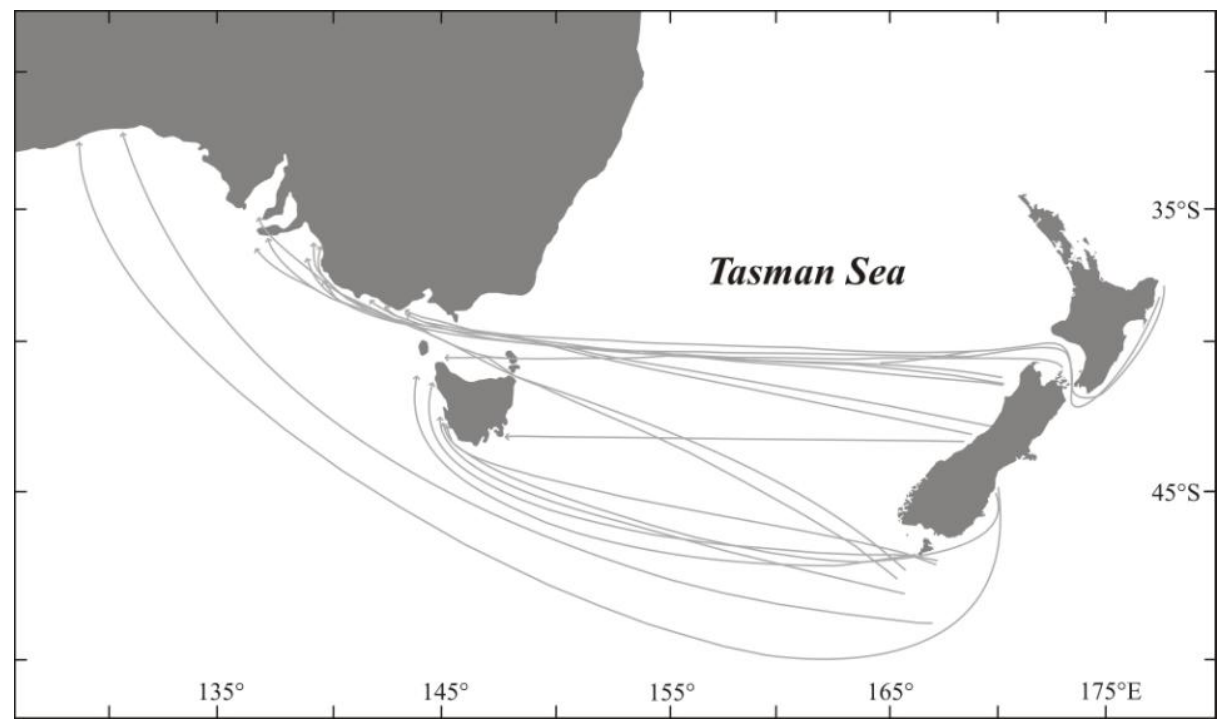

Figure 1.1: Movements of New Zealand tagged school shark, Galeorhinus galeus to Australia adapted from Hurst et al. 1999.

The migration patterns of G. galeus in southern Australia waters can be inferred from long term tagging data (Figure 2.2). Pregnant females move into the nursery areas along the southern Australian coast. After parturition, females leave the nursery, whereas newborn sharks $(0+$ years $)$ remain around local areas before moving 
into deeper coastal waters during winter months (July-September) (Olsen 1954, 1984). The juveniles return to their former nurseries during the next spring or move to adjacent estuaries and bays. As a consequence, mixing of the juvenile population occurs among several nursery areas (Olsen 1954, 1984, Walker 1999). Some individuals $(2+$ years $)$ showed random patterns of migration in open waters, where they mix with other stocks; for example, a male of $58.5 \mathrm{~cm}$ TL moved $1120 \mathrm{~km}$ in 544 days from Pittwater (Tasmania) to Kangaroo Island (South Australia) (Olsen 1954).

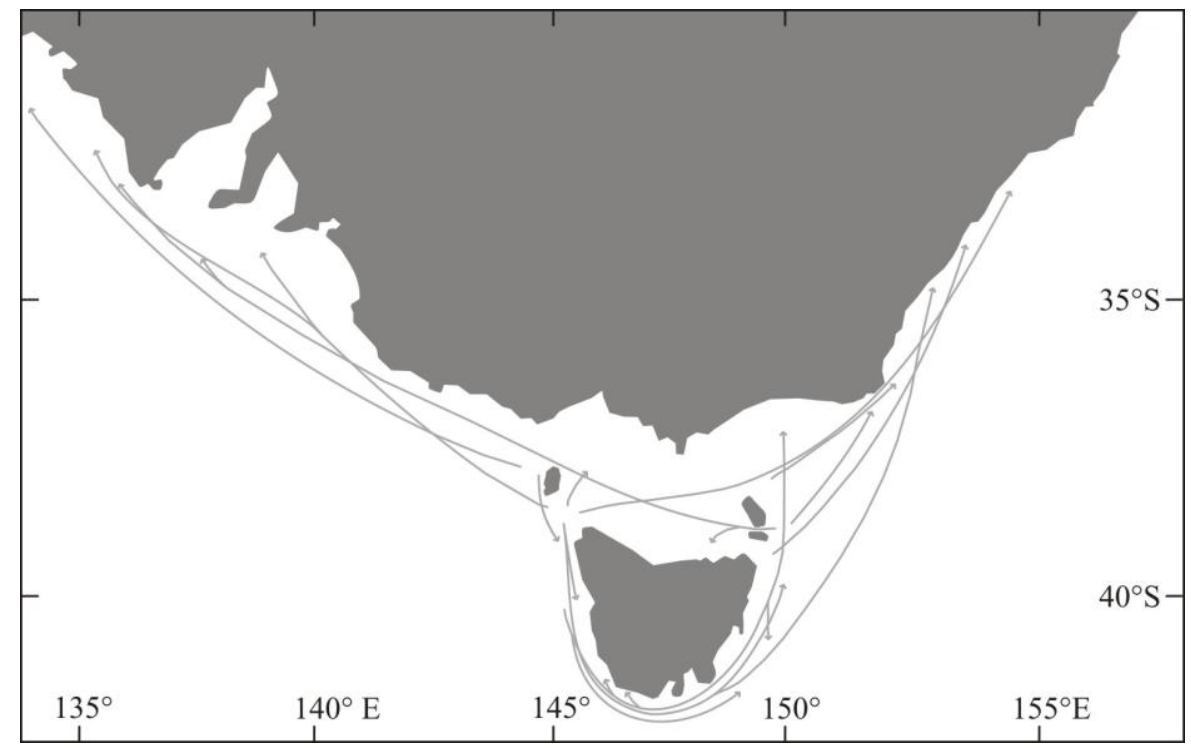

Figure 1.2: Movements of Australian school shark, Galeorhinus galeus within Australian waters adapted from Olsen 1984.

Adults tend to range more widely than juveniles. Adults migrate inshore in summer and offshore during winter, and also move in schools to the warmer northern waters of South Australia and New South Wales in winter before returning south in late spring (Olsen, 1954). The shark usually occurs in small schools segregated predominantly into individuals of the same sex and age (Last \& Stevens, 2009). Conversely, mixing of males and females occurs in deep waters of the continental shelf, coinciding with the beginning of the mating season in May-June (Olsen 1984). In addition, aggregations of pregnant females in different stages of gestation have been found in waters of the Great Australian Bright (Walker 2000, Walker 2005, Walker et al. 2008). These findings regarding adult movement along with records from fishing surveys, suggest that mature female G. galeus remain in South 
Australian waters during the gestation period. After this time, they start the reverse migration to the nursery areas in Victoria and Tasmania to give birth.

Pupping areas are typically harbours, shallow bays and sheltered coasts (Olsen 1954, 1984, Paul 1988, Paul \& Sanders 2001). The location of the most important pupping areas in New Zealand is unknown. However, trawl surveys suggest they may be around the east and west coast of the North and South Island except northern of North Island and southern of South Island, and the central-south coast of the South Island (Hurst et al. 1999). By contrast, several nursery areas are well described in Victorian and Tasmanian waters i.e. Port Phillip, Port Sorell, St. Helen, Pittwater (Olsen 1954, 1959, 1984, Stevens \& West 1997, TSSC 2009). However, due to the strong fishing pressures on G. galeus populations in southern Australian waters and the ongoing habitat degradation in inshore nursery areas, the abundance of newborns and juveniles has declined in coastal waters off Victoria and Tasmania.

\subsubsection{New Zealand and Australasian Galeorhinus galeus Fisheries}

Fishing effort on G. galeus populations has been well documented in the past (e.g. an apparent collapse in California, Stevens 2000). This species has been fished in Australia since the mid-1920s and in New Zealand since the early 1940s by separate commercial fisheries. Australian fisheries began in 1927 in the eastern Bass Strait and spread to eastern South Australia and Tasmania during the 1940s. During this time, demersal longlines were used to target large mature $G$. galeus, which increased mortality of sub-adults (Olsen 1959, Walker 1999, Stevens 2005). As a result, the catch rate in southern Australia declined by more than 50\%. The fishing impact was reflected in the catches of pups as well e.g. an $80 \%$ decline in Port Phillip Bay, Victorian waters (Olsen 1984, 1959, Walker 1998, 1999). Indeed, as noted by Olsen (1984) Pittwater and Port Sorell (Tasmanian waters) also indicate a decline in catch rates (Stevens 2005).

In the early 1960s, the fishery grew rapidly, peaking at over $3756 \mathrm{t}$ (Walker 1999) due to the demand for shark meat in Victoria. Gillnetting was implemented in 1964, and by the early 1970 s, most of the catch was taken by this gear. However, demand for the fish declined due to concern about high levels of mercury in the flesh, 
resulting in the banning of fishing for large G. galeus. As a result, fishing effort shifted to the gummy shark (Mustelus antarcticus). After the lifting of the ban on $G$. galeus, catches of that species increased again, peaking at $2000 \mathrm{t}$ in 1986 (Walker 1999, AFMA 2009). Currently, G. galeus are not targeted, but are landed as bycatch in Australian M. antarcticus fisheries (Stevens 2005, McLoughlin 2007).

Australian G. galeus fisheries have reduced the stock to less than $15 \%$ of initial biomass, raising serious concerns (AFMA 2009, 2010a, DSEWPaC 2012). The depletion of the stock has led to a progressive reduction in the TAC (total allowable catch) from $800 \mathrm{t}$ in 2002 to $240 \mathrm{t}$ in 2010 (AFMA 2010b). It has been listed under the "conservation dependent" category of the Environment Protection and Biodiversity Conservation Act 1999 (EPBC Act) which allows for the G. galeus shark rebuilding strategy plan (TSSC 2009). The rebuilding strategy consists of several management measures and responses designed to recover the population to a given target biomass within a biologically reasonable timeframe (AFMA 2009, TSSC 2009). The management measures include: limited entry, area closures, gear restrictions, and minimum legal-size limits (TSSC 2009). Despite these measures, there is little evidence of stock recovery and as recently as September 2010, additional measures were being considered, including catch restrictions of co-occurring target species and area closures (AFMA 2010b).

In contrast, the New Zealand G. galeus fishery is much larger than the Australian one, and it is currently considered to be more sustainable. The fishery started with Maori using the meat, oil, and skins during pre-European times; however there is no quantitative information about their catches (Francis 1998, MPI 2012). During the 1940s, factory companies processed the livers of G. galeus, however the catches were not recorded. After this, the New Zealand market started to export $G$. galeus meat to Australia. The landings increased to 300-600 t from 1957 to 1971, but the banning of the meat's importation to Australia resulted in a reduction of the New Zealand fishery (Francis 1998, Paul \& Sanders 2001). However, the fishery increased rapidly from $500 \mathrm{t}$ to $5,600 \mathrm{t}$ between 1979 and 1984 due to the re-opening of the Australian market. The New Zealand TAC was set at 2590 t, which was more than $50 \%$ of the average annual catch for the previous three years. Between 1995 and 1996, the total landings rose to $3387 \mathrm{t}$ and the TAC (3106 t) was exceeded for the first time (Francis 1998). Since then, the average catch in New Zealand G. galeus fishery 
has consistently exceeded $3000 \mathrm{t}$ (MPI 2012). However, there is a concern about the sustainability of the fishery due to high TAC levels through recent decades. As a consequence, is has been proposed that fishing pressure on adults, especially large mature females, should be minimised to maintain the productivity of the fishery.

There are few recreational restrictions on the G. galeus fishery in New Zealand. The minimum mesh-sizes for gillnetting are $125 \mathrm{~mm}$ for Auckland Fisheries Managemnet Area (AFMA) and $150 \mathrm{~mm}$ for Central, Southland and Challenger Fisheries Areas. The minimum dragnet mesh size is $100 \mathrm{~mm}$ for AFMA. Additionally, several general restrictions apply for commercial gillnets and longlines such as: limits on the length of gillnets, number of hooks per line, number of longlines, soak time, proportion of a bay or estuary that can be blocked by a gillnet, and areas that can be fished (Walker et al. 2006). Recreational daily bag limits of mixed species apply to G. galeus as well i.e. person can catch 20-30 fish for the northern, central and southern regions of the country (MPI 2012).

In conclusion, given the commercial importance of G. galeus stock and the concern about population levels (both at trans-Tasman and local scales), knowledge of stock structure is essential for the effective management of the fisheries in New Zealand and Australia. This is particularly important given the major depletion of stocks in Australia and their conservation status on the IUCN Red List as vulnerable in Australia and near threatened in New Zealand (Walker et al. 2006). 


\subsubsection{Goals of the thesis research}

Studies of G. galeus using tagging and early genetic markers have supported the idea of large genetically homogeneous population in New Zealand and Australian waters. However, the question of whether this species comprises a single population or multiple sub-populations has not being properly resolved. The overall goal of this thesis research is to determine the levels of genetic variation and differentiation within and among G. galeus populations in New Zealand and Australia by using mtDNA and nDNA molecular markers. Samples from Chile have also been included in the analysis due to opportunity to cover the entire distribution of the genetic variation of the species within South Hemisphere (i.e. no New Zealand and Chilean samples were reported in Chabot \& Allen 2009).

Three categories of genetic structure described in Laikre et al. (2005) will be tested in order to classify the genetic connectivity of G. galeus. The categories of genetic structure are 1) single panmictic stock, 2) isolation by distance, and 3) discrete stocks. The first category involves genetic flow that is so extensive that the entire population is not reproductively isolated, behaving as a single stock. The second category involves genetic variation more or less continuously over space. The gene flow among nearby individuals is larger than those separated by long distance, resulting in successive genetic change over geographic distance defined as "Isolation by distance" (IBD). The last category is characterised by a very small amount of migration between stocks, which could be sufficient to differentiate between closely geographic regions. Two hypotheses of population genetic structure were tested in this thesis research:

$H_{01}$ : There is a single panmictic stock in the New Zealand and Australia region.

$H_{\mathrm{A} 1}$ : There are separate populations in New Zealand and Australia due to isolation-by-distance and/or discrete differences.

If $H_{01}$ is rejected, a further set of hypotheses were tested:

$H_{02:}$ There are panmictic stocks within New Zealand and Australia.

$H_{\mathrm{A} 2}$ : Discrete separate sub-populations occur within New Zealand and Australia due to isolation-by-distance and/or discrete differences. 
Chapter 2 aims to describe and contrast the patterns of genetic connectivity inferred from the mtDNA control region of three shark species (G. galeus, Squalus griffini and Sphyrna zygaena) that are distributed in South Pacific Ocean. Two additional species were included (S. griffini and S. zygaena) in order make a comparison with the genetic structure of $G$. galeus, and therefore compare the population patterns over large and small comparable spatial distances. In Chapter 3, I examine the use of next generation sequencing technology (NGS) to develop microsatellite markers for G. galeus. Nineteen putative microsatellite loci were tested in three collection sites from Australasia region and Chilean ones as well, in order to determine their utility to test for genetic differentiation. Additionally, nine other microsatellite loci designed by Chabot \& Nigenda (2011) were integrated as well to this Chapter. In Chapter 4, I studied the level of genetic variation inferred by microsatellite DNA markers for $G$. galeus for adult collections in order to test for spatial population structure. Coalescent-based methods were carried out to estimate migration rates inferred by mitochondrial DNA (CR) and microsatellite loci in order to assess population connectivity of this species across the Tasman Sea. In Chapter 5, I investigate evidence of multiple-paternity among embryos in G. galeus by assessing the levels of relatedness of progeny using microsatellite DNA markers. Five "families" (mothers and litters) were sampled and a parentage analysis was conducted using six microsatellite loci. Finally, Chapter 6 aims to integrate the information generated in the previous chapters in order to synthesise the implications of this research; the results for G. galeus are compared with other shark species, and possible future research directions are discussed. 


\section{Chapter 2}

Contrasting the population genetic structures of the school shark (Galeorhinus galeus), northern spiny dogfish (Squalus griffini) and smooth hammerhead (Sphyrna zygaena) using mitochondrial DNA control region sequences

\subsection{Abstract}

The three species of shark that have similar distributions in the Tasman Sea and South Pacific Ocean were compared using mitochondrial DNA control region (CR) sequencing to undestand their population genetic structures. DNA sequences between 900 and 600 base pair of the CR were determined from 475 Galeorhinus galeus, 145 Squalus griffini and 156 Sphyrna zygaena individuals. Relatively high levels of haplotype diversity $(h)$ were found for G. galeus $(h=0.75)$ and S. griffini $(h=0.70)$, compared with $S$. zygaena populations $(h=0.62)$, but relatively low level of nucleotide diversity $(\pi)$ were found for $G$. galeus $(\pi=0.015)$ and S. griffini $(\pi=$ $0.023)$, in comparison with $S$. zygaena populations $(\pi=0.024)$. AMOVA analysis and pairwise genetic differences $\left(\Phi_{S T}\right)$ for $G$. galeus showed a significant level of genetic differentiation between a New Zealand/Australian population and the Chile population. Within the New Zealand and Australia region there was a lack of genetic differentiation, suggesting a single panmictic stock mediated by high levels of female gene flow. Evidence of two genetically distinct populations of the endemic New Zealand species S. griffini was found when comparing samples from the Kermadec Islands ( 600 nautical miles northeast of Auckland), and a combination of samples from sites at Gisborne, Westland, and Chatham Rise (mainland stocks). For $S$. zygaena, a weak level of genetic structure was found across the South Pacific Ocean, but there was no evidence of genetic differentiation between sampling sites in New Zealand and Australia, and between Chile and Ecuador. The mitochondrial DNA control region data support the suggestion of join management of the Australian and New Zealand G. galeus stocks, but further consideration should be given to managing the New Zealand G. galeus as two separate stocks. Similarly there is support for 
considering New Zealand S. griffini as northern and southern stocks. In contrast to the other two species, S. zygaena stock structure should be more thoroughly investigated using other mitochondrial DNA markers and/or with a high level of resolution as microsatellites (sampling more independent regions on the genome). 


\subsection{Introduction}

Population genetic markers are important tools for defining the reproductive units within a fishery (stocks) and monitoring the levels of genetic variation within an exploited species. Genetic data enable the identification of fish stocks (both spatially and temporally), providing an indirect estimate of the migration rates among fishery sites (Nielsen et al. 2009). However, genetic information has only slowly been integrated into stock assessment models and it is often used in combination with other approaches such as direct stock identification methods (e.g. tagging programmes) (Waples et al. 2008, Dichmont et al. 2011). Population genetic methods are used to identify groups of individuals within a species that share the same reproductive process, which can be used as the basis for defining management units (Laikre et al. 2005). Furthermore, understanding and managing the genetic constituents of a population is important for the long-term persistence and adaptability of a commercial species (Allendorf et al. 2008). Managing genetic diversity is particularly important for mitigating the risks of inbreeding and reduced adaptability that could be caused when stocks are fished down to small sizes (Frankham 2002).

The sustainability of shark fisheries has been a concern for the last three decades. Many species have become overexploited because of the high demand for meat and fins for human consumption (Musick \& Bonfil 2005). Moreover shark species often have low management priority when resources are limited and basic fisheries data are lacking (Stevens et al. 2000). Effective fishery management relies on a stock assessment, which requires a good understanding of a range of biological parameters such as the number of reproductively independent of units within a fishery and their structure (Carvalho \& Hauser 1994, Grant \& Waples 2000). Traditionally, large shark species were thought to be highly mobile and typically compressed one large and widely distributed population, whereas and smaller species were thought to have a lower power of dispersal and might be comprised of several structured populations (Musick et al. 2004). However, tagging studies have shown that this generalization is not often true and many species migrate over reasonably large distances (Hurst et al. 1999, Kohler \& Turner 2001, Walker et al. 2008).

Despite the high dispersal potential of many shark species, recent genetic studies have shown that coastally distributed species, particularly of the family 
Carcharhinidae, lack significant amounts of gene flow across ocean basins (Duncan et al. 2006, Keeney \& Heist 2006, Schultz et al. 2008, Chabot \& Allen 2009, Portnoy et al. 2010). Some species have complex migration patterns and their dispersal pattern can vary significantly during their life cycle due to mating, pupping, and maturation behaviors (Hueter et al. 2005). Where known these life history parameters biological features are taken into account when attempting to identify stock structure.

Galeorhinus galeus is a commercially fished shark species that is widely distributed in temperate waters (Last \& Stevens 2009). It occurs throughout New Zealand waters, but in Australia it is restricted to southern waters from Moreton Bay (southern Queensland) to Cape Leeuwin (Western Australia), including Lord Howe Island and Tasmania (Last \& Stevens 2009). Galeorhinus galeus has been managed separately in New Zealand and Australia, and the average annual catch in New Zealand currently exceeds $3000 \mathrm{t}$, whereas in Australia it is limited to $240 \mathrm{t}$ (AFMA 2010, MPI 2012). The Australian management authorities were concerned about the sustainability of the G. galeus fishery and they introduced a long-term fishing plan to rebuild the stock within a biologically reasonable timeframe (AFMA 2009). However, G. galeus stocks in Australia still remain unacceptably low. The limited reproductive potential and the trend of declining population size worldwide suggest that this species is overexploited and it has been listed as a globally Vulnerable species by the IUCN (Walker et al. 2006).

Tagging studies have shown that the Australasian G. galeus are long-lived and highly mobile within and between New Zealand and Australia (Olsen 1953, 1954, Stanley 1988, Paul 1988, Walker 1989, Francis \& Mulligan 1998, Hurst et al. 1999, Walker et al. 2008, Francis 2010). For example, one female released in the Great Australian Bight was recaptured 3,500 km away (4.1 years later) between the North and South Islands of New Zealand. It was released again and recaptured off Australia $2,600 \mathrm{~km}$ away (2.8 years later; Walker et al. 2008). The maximum distance travelled was $4,940 \mathrm{~km}$ (over 9.6 years; Hurst et al. 1999). The overall findings of the tagging studies have been used to support the hypothesis of a single Australasian stock in the fisheries assessments of G. galeus (Punt et al. 2000, Walker et al. 2008).

The patterns of gene flow among G. galeus populations sampled in different ocean basins have been documented using Restriction Fragment Length 
Polymorphisms (RFLPs) of the mtDNA and the direct DNA sequencing of the mtDNA control region (CR). These studies have shown a significant level of genetic differentiation among ocean basins (Ward \& Gardner 1997, Chabot \& Allen 2009). A weak level of differentiation was reported between the east coast of New Zealand and west Tasmania in Australian populations (Ward \& Gardner 1997). These genetic markers did, however, have several limitations; the RFLP technique has a very limited ability to detect genetic variation, the sample sizes were uneven, and the sampling did not cover the entire distribution of the species within the region (e.g. no New Zealand samples were reported in Chabot \& Allen 2009). Despite the drawbacks of the tagging and genetic studies, the results have been used to support a model of separate but genetically homogeneous stocks of G. galeus in New Zealand and Australia. The question of whether Australasian G. galeus comprises a single stock or multiple substocks has not been properly resolved.

The northern spiny dogfish (Squalus griffini) is a New Zealand endemic benthonic shark species with a distribution that overlaps with G. galeus although more patchy in it distribution. Squalus griffini occurs along the continental margin off the west coasts of the North and South islands from about $34^{\circ} \mathrm{S}$ to $44^{\circ} \mathrm{S}$. Off the east coast, it has been distributed southward to almost $45^{\circ} \mathrm{S}$, although infrequently caught south of $38^{\circ} \mathrm{S}$, with the exception of Chatham Rise between $42^{\circ} \mathrm{S}-44^{\circ} \mathrm{S}$, and $175^{\circ} \mathrm{W}-$ $179^{\circ} \mathrm{W}$ respectively (Anderson et al. 1998, Duffy \& Last 2007). This species has also been recorded north of New Zealand on Wanganella Bank and Norfolk Ridge (to at least Norfolk Island), Kermadec Ridge (to at least Raoul Island, $29^{\circ} \mathrm{S}$ ) and the Louisville Ridge. The distribution data suggest that the Kermadec Islands and Chatham Islands $S$. griffini populations are somewhat isolated from the mainland populations. However, the population genetic structure of this species has not been studied.

Squalus griffini is often caught as bycatch in non-directed fisheries. The total catch is small and it is not in the Quota Management System (QMA) in New Zealand. There is a scarcity of data available about commercial catches and very little information about life cycle and habitat ecology of S. griffini. The un-standardized annual catch-per-unit-effort (CPUE) suggests large parts of the distribution range of the species remain unfished or only lightly commercially fished (Duffy 2011). Research trawl surveys off the west coast of the South Island showed no particular 
downward trends in relative biomass (Cavanagh \& Lisney 2003), which put it in the Least Concern category of species by the IUCN (Duffy 2011). However, it is not known whether set net and bottom longlines fisheries may cause localised stock depletion. Squalus griffini is assumed to have low reproductive potential, comparable to the closely relates mitsukurii species (Duffy 2011).

The smooth hammerhead shark (Sphyrna zygaena) is a circumgloballydistributed species, which occurs in coastal-pelagic and semi-oceanic environmental habitats. It is found in temperate and tropical waters from shallow (less than $20 \mathrm{~m}$ ) to $200 \mathrm{~m}$ along the continental shelf. However, the full range of the species in tropical waters is not known due to its confusion with Sphyrna lewini. In the southwest Pacific it is distributed in Australia from southern New South Wales to central western Australia and New Zealand, including Lord Howe and Kermadec Islands, while in the southeastern Pacific it is distributed from Ecuador to Chile (Last and Stevens 2009). Tagging data suggest that juvenile and sub-adult $S$. zygaena make large-scale directional movements in South Africa $(\sim 300 \mathrm{~km})$ in response to seasonal sea surface temperature changes (Diemer et al. 2011). A genetic survey of the mtDNA control region reported population subdivision among four geographic regions with little evidence of gene flow among the Atlantic, North Pacific, southeast Pacific and IndoPacific oceans (Testerman et al. 2008).

Sphyrna zygaena has been listed as a globally Vulnerable species by the IUCN (Casper et al. 2005) because of reports of declining population sizes and life history characteristics that make it susceptible to over-exploitation. In New South Wales (Australia), this species along with $S$. lewini and $S$. mokarran, is caught by a commercial line fishery and recreational game fishery. The majority of the hammerhead catch is comprised of S. zygaena and S. lewini (Scandol et al. 2008). The total commercial hammerhead landings peaked in 1993 at $15 \mathrm{t}$ and in 1994 at $7 \mathrm{t}$ and annual commercial catches have averaged $\sim 3 \mathrm{t}$ over the last five years (Casper et al. 2005). Sphyrna zygaena has also been reported in set net catches in the New South Wales beach protection programme (Krough 1994), which uses large mesh gillnets as a protective measure for beach users. In New South Wales, hammerheads (mostly $S$. zygaena) made up nearly $50 \%$ of the beach protection catch of 4,715 sharks in the period from 1972-73 to 1989-90 (Reid \& Krough 1992, Casper et al. 2005). This species is an important bycatch species in the West Australian demersal gillnet 
fishery, but the relatively low numbers caught have not caused a significant impact on population numbers.

In New Zealand waters, S. zygaena is reasonably abundant around the northern North Island and it is the most common shark species reported in aerial surveys conducted along the northwest coast (Casper et al. 2005, Francis 2010). Neonates and small juveniles are commonly found in shallow coastal waters of the northern North Island, but are absent further south. They are caught as bycatch in commercial gillnet fisheries for flatfish and taken by trawlers and Danish seiners. Juveniles caught in gillnets frequently die before they can be released and a lot are finned as well (Smith \& Benson 2001). Adults are mainly taken by game-fishers as bycatch, the annual catch is small and many of the sharks are released alive (Casper et al. 2005, Francis 2010). Currently, S. zygaena is the only representative of Sphyrna in New Zealand waters, which make easy the identification and information from catches. However, species-specific information about catches is very limited in Australian waters because they are often grouped together under a single category (Sphyrna group).

In Ecuador and Chilean waters, S. zygaena is caught as bycatch by artisanal and industrial-fishing vessels using surface longlines and drift gillnets. In Ecuador, $S$. zygaena represents $11 \%$ of the total chondrichthyan landings, which typically contain high numbers of juveniles (Casper et al. 2005). In Chilean waters, there is no record of catches and/or landing for S. zygaena. However, the majority of bycatch are processed in Iquique and Caldera ports for its meat and fins (northern of Chile) (Lamilla et al. 2005). The meat is usually traded locally for human consumption, whereas the fins are dried and processed to be exported to the Asian market, mainly Hong Kong (Hernandez et al. 2008).

The aim of this Chapter was to determine the levels of genetic variation and differentiation using mtDNA CR sequences among populations of three shark species (G. galeus, S. griffini and Sphyrna zygaena), which are co-distributed in the southwestern and southeastern Pacific Oceans. Two additional species were included (S. griffini and S. zygaena) in order to make a comparison with G. galeus genetic structure and population patterns over similar spatial distances. The genetic results are used to identify the putative stock structure of these species. Furthermore, non-coalescent and coalescent approaches were used to test for non- 
equilibrium population dynamics and make inferences about their historical genetic effective population sizes. 


\subsection{Methods}

\subsubsection{Sampling collection}

Tissue samples of G. galeus were taken from individuals utilizing two different sampling approaches. The first method targeted adult sharks from three geographic regions: New Zealand, Australia and Chile. Samples were collected from four areas around New Zealand (NZ): East of North Island (ENI), Kaikoura (KAI), Chatham Rise (CHA) and Solander Islands (SOL) (Figure 2.1A); and six locations from Australia (AUS): Shoulder (SHO), Great Australian Bight (GAB), Eyre (EYR), King (KIN), Tasmania (TAS) and Frederich Bay (FRE) (Figure 2.1A). Due to geographic proximity, small sample size and lack of genetic differentiation, samples from SHO and GAB, and EYR and KIN were pooled together, respectively for analysis purposes. Thus SHO and GAB was named SOU, and EYR and KIN became POR (Figure 2.1A). Additionally, G. galeus fin samples were collected from a shark fin warehouse in Santiago (SAN) from Chile (CHI), which were previously used for an identification genetic study of shark species involved in the Chilean fin trade (Hernandez et al. 2009) (Figure 2.1B). Adult collections were coordinated with on board observers and fisheries biologists from the Ministry of Fisheries and National Institute of Water and Atmospheric Research (NIWA) in New Zealand, and Commonwealth Scientific and Industrial Research Organization (CSIRO), Department of Primary Industry, and Marine and Freshwater Fisheries Research Institute in Australia.

The second method targeted pups in New Zealand and Australian pupping areas. During this study, six locations were sampled in New Zealand: Kaipara Harbour (KAH), Manukau Harbour (MAN), Napier (NAP), Kapiti Coast (KAP), Mapua (MAP), and Golden Bay (GOL); and one in Tasmania: Upper Pittwater (UPW) (Figure 2.2). The surveys were coordinated with scientists from Department of Conservation (DOC), New Zealand National Aquarium-Napier, Mapua AquariumNelson, Victoria University of Wellington from New Zealand, and CSIRO (for collections from Tasmanian waters). Captures were made by demersal longlines. Newborn young and juveniles were caught and released back into the water within a maximum period of two hours from capture. It is known from previous studies that 
handled young G. galeus are highly active and in good condition up to two hours after capture on a longline (Stevens \& West 1997).

Additionally, another group of samples were obtained from a previous $G$. galeus population genetic study, which utilized allozymes and RFLP-mtDNA (Ward \& Gardner 1997). These samples were collected in 1993-1994 from global sampling locations and extensive sampling from New Zealand and Australian waters. Unfortunately, only samples from Tasmania (Australia) were recovered from the remaining frozen tissues stored at CSIRO. Since many of the samples were unlabelled or labelled improperly, it was only possible to identify fewer than half of the samples by location according to the dataset provided by Bob Ward (CSIRO). Therefore, samples of six nurseries areas from Tasmanian waters were used: Upper Pittwater (UPW), Blackman Bay (BLA), Norfolk (NOR), Ralph Bay (RAL), Isthmus Bay (ITH), and Swansea (SWA) (Figure 2.1). 


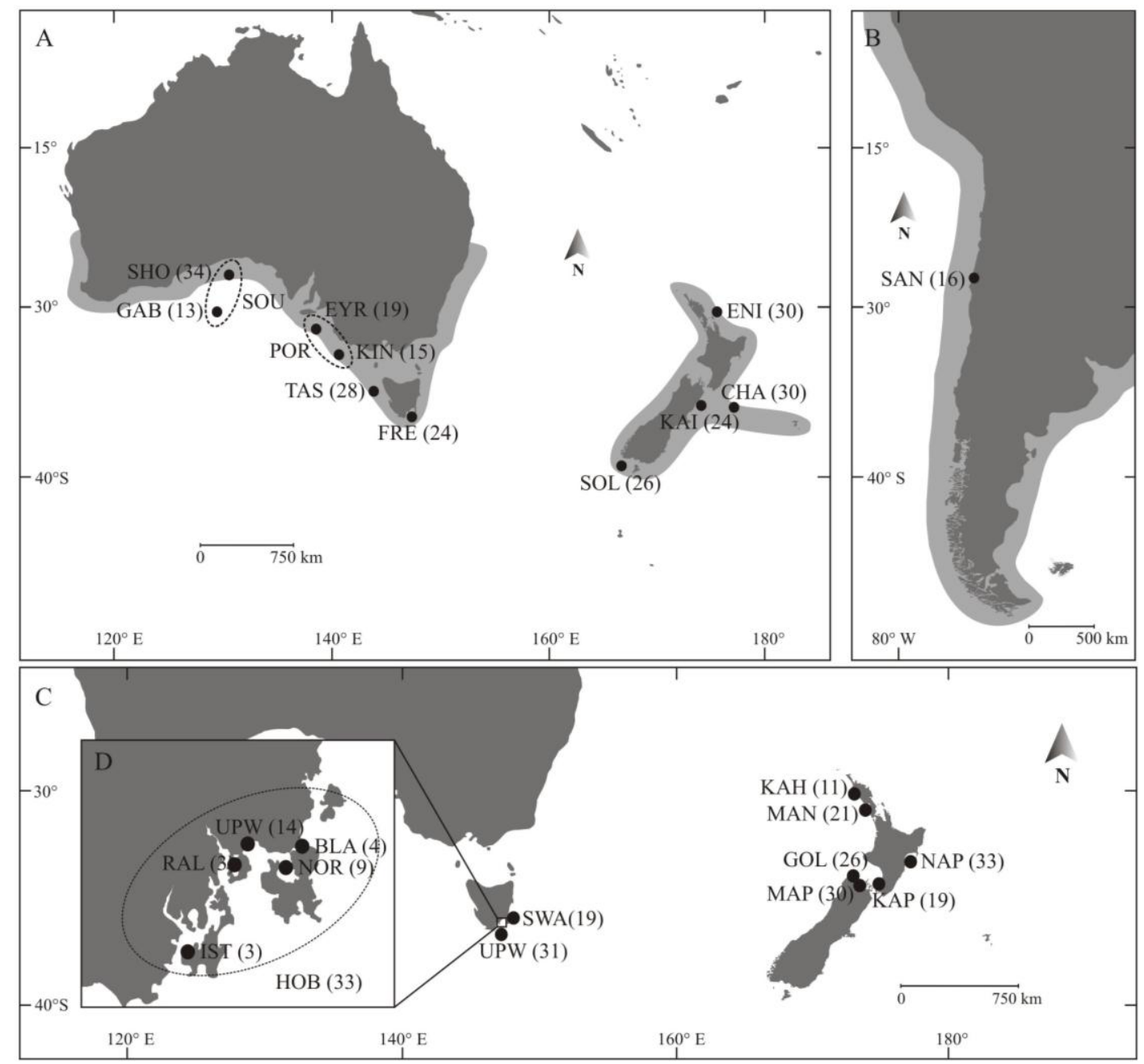

Figure 2.1: Sampling sites, sample sizes (in parentheses), and geographic distribution (shaded area) of G. galeus collected from New Zealand (NZ), Australia (AUS), and Chile (CHI). A. and $\mathbf{B}$. Sites where adult individuals were sampled. The locations are: ENI (east North Island, NZ), CHA (Chatham Rise, NZ), KAI (Kaikoura, NZ), SOL (Solander Island, NZ), SHO (Shouder, AUS), GAB (Great Australian Bight, AUS), EYR (Eyre, AUS), KIN (King, AUS), TAS (west Tasmania, AUS), FRE (Frederich Bay, AUS), and SAN (Santiago, CHI). The SHO and GAB (SOU), and EYR and KIN (POR) were considered as one location in the analyses (see text details). C. Sites where pups were sampled across the Australasian region. The locations are: KAH (Kaipara Harbour), MAN (Manukau Harbour), NAP (Napier), KAP (Kapiti Coast), MAP (Mapua), GOL (Golden Bay), UPW (Upper Pittwater), STA (St. Helens), and SOR (Port Sorell). D. Individuals collected from Tasmanian waters during 19931994. The locations were: Upper Pittwater (UPW), Blackman Bay (BLA), Norfolk (NOR), Ralph Bay (RAL), Isthmus Bay (ITH), and Swansea (SWA). These locations, excluding SWA, were pooled as HOB (Hobart area) for descriptive genetic diversity, haplotypes distribution, and temporal AMOVA. 
Squalus griffini tissues samples were taken from Kermadec Islands (KER) in the North Island, Gisborne (GIB), Westland (WES) and Chatham Rise (CHA) from the South Island in New Zealand (Figure 2.2A). Samples were collected during fishing surveys that were organized by scientists from DOC, Te Papa Fish Collection, and the NIWA. During the same time, samples of S. zygaena were obtained from Australia, New Zealand, Chile and Ecuador (Figure 2.2B). The samples were contributed by scientists of SARDI Aquatic Sciences from Adelaide, South Australia, Fish Collection of Australian Museum from Sydney, DOC from New Zealand, and a researcher at Universidad Nacional Autónoma de México from Ecuador. Chilean samples were obtained from dry fins sampled from two commercial warehouses of shark fins in Chile.

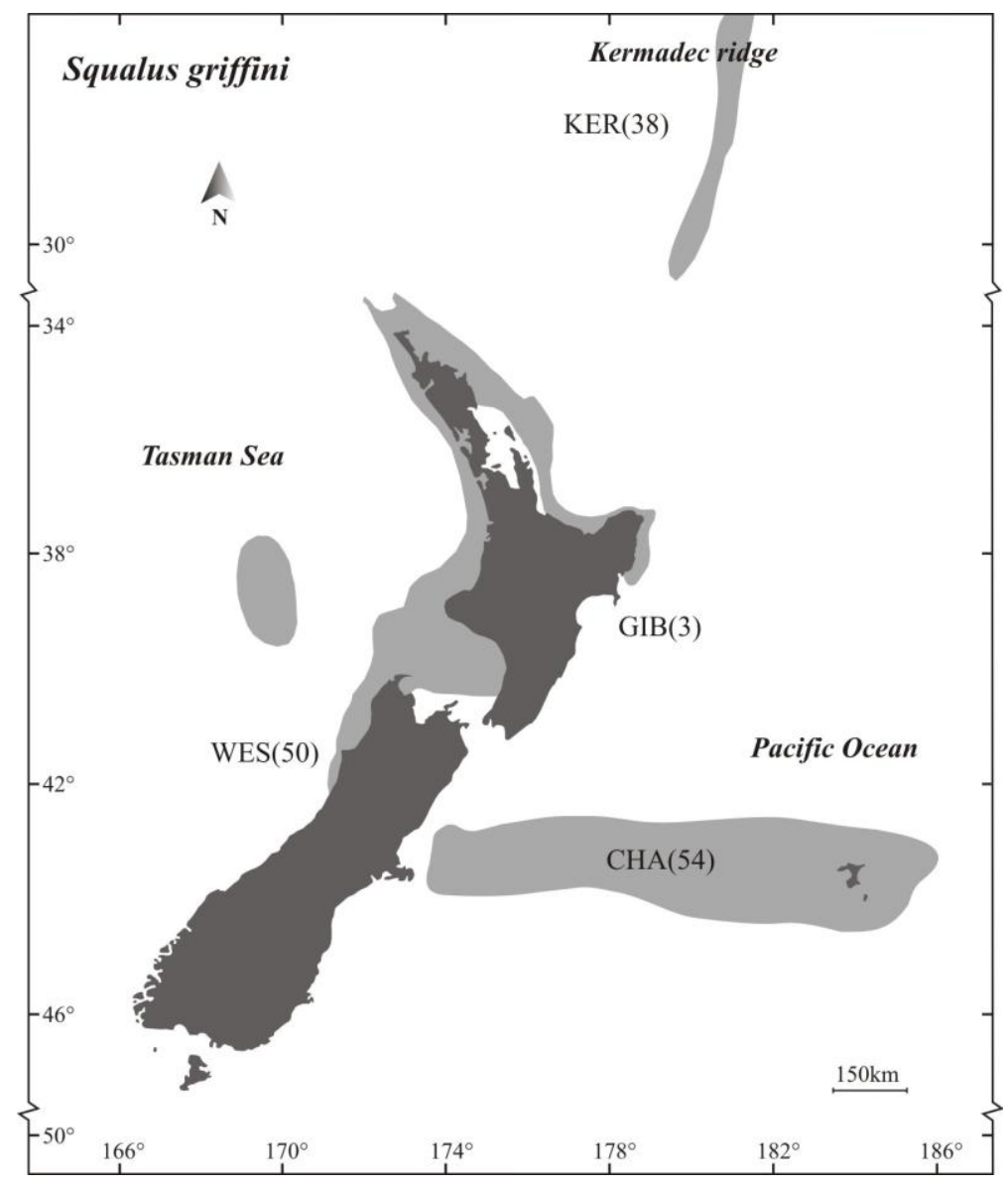

Figure 2.2: Sampling sites, sample sizes (in parentheses), and geographic distribution (shaded area) of S. griffini collected from New Zealand in GIB (Gisborne), WES (Westland), CHA (Chatham Rise), and KER (Kermadec Islands). 


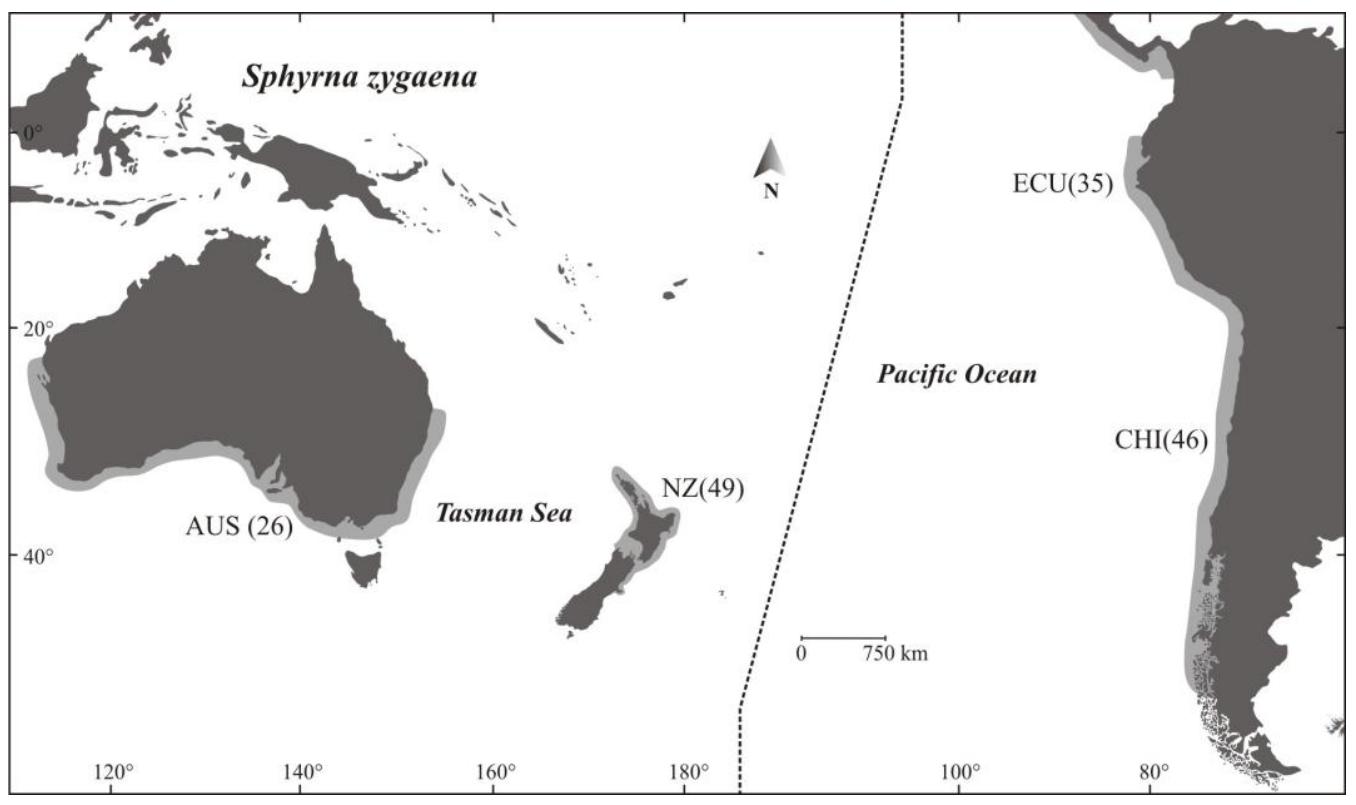

Figure 2.3: Sampling sites, sample sizes (in parentheses), and geographic distribution (shaded area) of $S$. zygaena collected from AUS (Australia), NZ (New Zealand), CHI (Chile), and ECU (Ecuador). 


\subsubsection{DNA Extraction, amplification and sequencing}

Genomic DNA was extracted from tissue samples using phenol-chloroform protocols (Sambrook et al. 1989). The CR of the mtDNA was amplified and sequenced by using species-specific primers designed in Primer 3 (Rozen \& Skaletsky 2000), implemented in Geneious Pro v5.5.2 (Biomatters Ltd.). Primers, approximate sizes of the amplified product and PCR conditions are detailed in Table 2.1. The resulting amplicons were sequenced from both forward and reverse directions during the initial data collection stage. Firstly, twenty sequences were sequences in both directions in order to evaluate the accuracity of forward and reverse sequences results. When DNA sequence methods were consistently only forward sequences were obtained for the remaining samples. PCR amplifications were carried out in $25 \mu 1$ volumes containing $67 \mathrm{mM}$ Tris- $\mathrm{HCl} \mathrm{Ph} \mathrm{8.8,} 16 \mathrm{mM}\left(\mathrm{NH}_{4}\right)_{2} \mathrm{SO}_{4}, 2.0 \mathrm{mM} \mathrm{MgCl}$, $20 \mathrm{mM}$ dNTPs, $1.5 \mathrm{mM}$ $\mathrm{MgCl}_{2}, 10 \mu \mathrm{M}$ of each primer, 0.4 units of BIOTAQ ${ }^{\mathrm{TM}}$ DNA Polymerase (Bioline) and $1 \mu \mathrm{l}$ of DNA (20-40 ng/ $\mu \mathrm{l}$ DNA concentration). PCR was performed in a TGradient Thermal Block (Biometra, Goettingen, Germany). Amplicons were visualised under UV-light after electrophoresis in a $1 \%$ agarose gel and staining with ethidium bromide. PCR products were purified using ExoSAP-IT kits and the DNA sequences were determined using an ABI 3730 Genetic Analyzer (Massey University). CR-mtDNA sequences were edited in Geneious Pro v5.5.2, and aligned using the Geneious Alignment option defined by default parameters (Drummond et al. 2011). In order to resolve any ambiguities and confirm the nucleotide calling, the chromatograms were thoroughly checked by eye. The CR-haplotype data from Chile were compared to other sequences reported from South American that were retrieved from Genbank (refer to Discussion for more details).

Table 2.1: Primer sequences and PCR conditions of CR for each shark species studied.

\begin{tabular}{llcc}
\hline Species & Primer sequences & Size (bp) & PCR cycling conditions \\
\hline Galeorhinus galeus & GGCR_F: 5'-CGAACCTAGCCCTTGGCCCAC-3' & 893 & $95^{\circ} 15^{\prime \prime}, 60^{\circ} 30^{\prime \prime}, 70^{\circ} 60^{\prime \prime}, 30$ cycles \\
& GGCR_R: 5' -ACGTCGGTCCTCGTTTTAGGGG-3' & & \\
Squalus griffinii & SGCR_F 5'-AGCCCACATATCCTAATATACCA-3' & 645 & $94^{\circ} 30^{\prime \prime}, 63^{\circ} 30^{\prime \prime}, 70^{\circ} 60^{\prime \prime}, 35$ cycles $^{\prime}$ \\
& SGCR_R 5'-GCGAATATTTTCCACACGCA -3' & & \\
Sphyrna zygaena & SPH_F 5'-ACCGGTTTTTGTACGTCAGT -3' & 760 & $94^{\circ} 30^{\prime \prime}, 63^{\circ} 30^{\prime \prime}, 70^{\circ} 60^{\prime \prime}, 35$ cycles \\
& SPH_R 5'-ACATTGGCCCTCGTTTTAGGGGT-3' & & \\
\hline
\end{tabular}




\subsubsection{Data analyses}

\subsubsection{Genetic diversity and population structure}

Descriptive genetic diversity statistics were estimated as the number of segregation nucleotide sites $(k)$, number of haplotypes $(n)$, private haplotypes ( $p h$, haplotypes restricted to a single sample and not found elsewhere), haplotype diversity ( $h$, measure of the frequencies and number of haplotypes among individuals), and nucleotide diversity ( $\pi$, average weighted sequence divergence between haplotypes) for each population using ARLEQUIN 3.1.1 (Excoffier et al. 2005). To deteremine whether the sampling efforts were sufficient because of uneven sample sizes, rarefaction curves were estimated using ANALYTIC RAREFACTION 1.3 (UGA Stratigraphy Lab website; http://www.uga.edu/ strata/software/). Rarefaction curves were constructed by plotting the number of samples sequenced against the expected number of mitochondrial haplotypes.

An analysis of the molecular variance (AMOVA; Excoffier et al. 1992) was performed in ARLEQUIN 3.1.1. AMOVA analysis estimates the variance components and $\Phi$-statistics values partitioned at three levels of hierarchical subdivision: among groups $\left(\Phi_{C T}\right)$, within populations $\left(\Phi_{S T}\right)$, and the interaction of both (among+within, $\Phi_{S C}$ ) for CR-mtDNA sequence. Hierarchical AMOVA was performed to evaluate the effects of temporal and spatial population subdivision. For G. galeus, a temporal analysis was carried out on NZ and AUS adult collections that were grouped based on years of sampling. Thus, samples from 2007-2010 (ENI, KAI, CHA, SOL, SOU, POR, TAS, and FRE), and also AUS samples from 1994 (SWA and HOB) were pooled together. Secondly, two spatial analyses were undertaken to test the hypothesis of panmixia among collections from: 1) NZ, AUS and CHI, and 2) NZ and AUS. For S. griffini a spatial population structure analysis were undertaken, where the hypothesis of panmixia was tested among collections from GIB-WES-CHA (pooled together) and KER. For S. zygaena a spatial analysis was carried among collections from NZ-AUS (pooled together) and CHI-ECU (pooled together). The genetic distance matrix for AMOVA was estimated by pairwise differences and the significance levels of the variance components and $\Phi$-statistic values were tested by 20,000 nonparametric permutations (Excoffier et al. 1992). 
Additionally, pairwise genetic differences $\left(\Phi_{S T}\right)$ were estimated as indicators of gene flow between all populations for each species. Conventional $\Phi_{S T}$ that were based upon haplotype frequencies were computed in ARLEQUIN 3.1.1 with 20,000 permutations. Datasets were analysed separately by sampling design, for adult and pup G. galeus collections. In order to minimise type I error (probability of incorrectly rejecting a true null hypothesis), sequential Bonferroni correction of the significant values was estimated for the pairwise $\Phi_{S T}$ due to relatively high numbers of simultaneous comparisons (Rice 1989). To test for isolation by distance (IBD), linearised $\Phi_{S T}$ values were plotted against the natural logarithm of geographic distance (kilometres) among sampling sites. This analysis was only carried out on $G$. galeus and S. griffini populations. Geographic distances were estimated by ruler option in Google Earth v.6.2.2 (Google Inc.). The distance calculations consist of the shortest path, by sea, between any two sampling locations. Significance was evaluated for each location using a Mantel test as implemented in ARLEQUIN 3.1.1 using 20,000 permutations.

Haplotype genealogies were estimated for all the CR-mtDNA sequences obtained in this study. The reconstruction of genealogies was performed using phylogenetic algorithms in order to estimate the relationship between haplotypes without ambiguities or unresolved connection (Salzburger et al. 2011). A phylogenetic reconstruction was estimated with a maximum likelihood (ML) approach in the PHYML 3.0 (Guindon et al. 2010). The substitution model was set according to the highest log-likelihood obtained with JMODEL 0.1.1 (Posada 2008). The Jukes \& Cantor (JC69, 1969) was obtained as the most suitable model for $G$. galeus. Only the proportion of invariable sites (p-inv) was fixed (0.9030). The Hasegawa Kishino Yano (HYK $+\mathrm{G}, 1985)$ was preferred for S. griffini ( $p$-inv $=$ 0.8260) and HYK for S. zygaena. The rest of the parameters in the substitution model, including tree searching and branch support were set as defaults in PHYML 3.0. The ML tree was loaded in HAPLOVIEWER software (Salzburger et al. 2011), where the haplotype genealogy was reconstructed and edited. 


\subsubsection{Mutation-drift and migration-drift equilibrium}

Three different approaches were used to explore any signature of population expansion of the CR sequences for the shark species analysed: (a) neutrality test ( $D$ and $F_{s}$ ), (b) mismatch distribution analyses, and (c) Bayesian skyline plot (BSP). Computation of Tajima's $D$ (Tajima 1989) and Fu's $F_{s}(\mathrm{Fu} 1997)$ statistics and their significance values were tested by coalescent simulations under the infinite-sites model in ARLEQUIN 3.1.1. For neutral markers $D$ and $F_{s}$ tests, significant negative values (less than $p=0.02$ ) are expected in populations that have undergone recent population expansion. While, significant positive values are expected in populations that have recently experienced population contraction (Tajima 1989, Fu 1997). However, Fu's $F_{s}$ test is more sensitive than other tests at detecting the signal of population expansion (Fu 1997, Ramos-Onsins \& Rozas 2002). Mismatch distribution analyses were also performed to evaluate possible events of population expansion (Rogers \& Harpending 1992). This analysis represents the distribution of pairwise haplotype differences calculated from DNA sequences. The shape of the distribution indicates different scenarios in population history, in particular being influenced by episodes of population expansion (Jobling et al. 2004). Thus, a population that undergoes expansion tends to have a unimodal and smooth distribution of haplotype differences, whereas a multimodal and rough distribution reflects long term stability (Rogers \& Harpending 1992). The observed distribution was tested against models of constant population size and population growth-decline in DNASP 5.10 (Librado \& Rozas 2009). In order to distinguish between these two types of distribution a Harpending's raggedness index ( $H_{r i}$; Harpending, 1994) and sum of squared deviations $(S S D)$ were estimated to fit the sudden expansion model. Demographic parameters $\theta_{0}, \theta_{1}$ and $\tau$ were estimated from a mismatch distribution. These parameters were estimated in order to examine the changes in population size, where $\theta_{0}$ was the population size before expansion, $\theta_{1}$ was the population size after the expansion, and $\tau$ was the age of the expansion expressed in unit of mutational time. The time $(t)$ since population expansion occurred could then be estimated under the equation $\tau=2 \mu t$ (Rogers \& Harpending 1992), where $\tau$ represents the age of population mutational time, and $\mu$ the mutation rates of the sequence. The $\mu$ was defined as $2 \mu k$, where $\mu$ was mutation rates and $k$ was number of nucleotide base pair of CR-mtDNA analysed. The CR mutation rate of the G. galeus is unknown; however, 
the substitution rate of five species of sharks is already estimated (reported in Dudgeon et al. 2012). According to this, the minimum and maximum values of calibrated mutation rates of CR $4 \times 10^{-9}$ and $2.15 \times 10^{-9}$ substitution/sites/year were used to estimate $\mu$ (Duncan et al. 2006, Keeney \& Heist 2006). Additionally, the effective population size $\left(N_{e f}\right)$ was calculated using the estimates of co-ancestry coefficient $\left(\theta_{s}\right)$ obtained in ARLEQUIN 3.1.1. Thus the equation for $N_{e f}=\theta_{s} / \mu(\mu$ calculation are described above) was used. The mutation rates to estimate $N_{\text {ef }}$ were the same as used to estimate the time since population expansion occurred.

Additionally, a Bayesian skyline plot (BSP) approach was implemented in BEAST 1.7.2 (Drummond \& Rambaut 2007). This technique permits the estimation of $N_{\text {ef }}$ through time and it does not require a specific demographic model (e.g. constant size, exponential growth, etc.) prior to the analysis as mismatch distribution does (Drummond et al. 2005). The BSP model generates posterior distribution of the effective population sizes through time using Markov chain Monte Carlo (MCMC) sampling. The substitution model $(\mathrm{HKY}+\mathrm{I})$ was selected according to the result of JMODEL 0.1.1 by using the corrected Akaike Information Criterion (AICc). In order to assess evidence of significant substitution rate variation among lineages in the CR sequences of each shark species, a likelihood-ratio test (LRT) was performed in PAUP* 4.0b10 (Swofford 2000). LRT tests were estimated both with and without enforcing substitution rate constancy. The LRT scores were compared under a Chi square $\left(X^{2}\right)$ test. A strict molecular clock approach assuming two different mutation rates of $4 \times 10^{-9}$ and $2.15 \times 10^{-9}$ substitution/sites/year was set (Duncan et al. 2006; Keeney \& Heist 2006). The coalescent Bayesian skyline process was chosen as tree prior assuming 10 grouped coalescent intervals for New Zealand and Australian pooled sequences, and 5 grouped coalescent intervals for Chilean sequences. A piecewise-constant skyline model was set as well (Drummond et al. 2005). A Markov chain was run for 500 million generations, and the first $10 \%$ were discarded as burnin. Inspection of results was conducted in Tracer 1.5.0 (Rambaut \& Drummond 2009) for checking whether performed runs have reached the stationary (> 200) of the posterior probabilities and effective sampling size (ESS) for all parameters. Finally, the BSP was reconstructed and plotted in Tracer 1.5.0 as well. 


\subsection{Results}

\subsubsection{Galeorhinus galeus}

An 893 bp DNA sequence of the 5' end of the CR was determined using DNA from 475 samples of G. galeus. The base composition of the aligned data set was $12.5 \%$ guanine, $33.1 \%$ adenine, $34.5 \%$ thymine, and $19.8 \%$ cytosine ( $32.3 \%$ GC content). A comparison of the sequences revealed 35 distinct haplotypes defined by 25 segregation sites, which were made up of 16 transitions and 11 transversions (GenBank Accession numbers KC118081-KC118115). The overall haplotype $(h)$ and nucleotide diversity $(\pi)$ were $0.746 \pm 0.015$ and $0.001 \pm 0.001$, respectively. Haplotype and nucleotide diversity were very similar in NZ $(h=0.735 \pm 0.032, \pi=$ $0.001 \pm 0.001)$ and AUS $(h=0.729 \pm 0.027, \pi=0.001 \pm 0.001$; Table 2.2) for the adult collection. However, the CHI $(h=0.800 \pm 0.089, \pi=0.002 \pm 0.001)$ area showed higher levels of diversity than NZ/AUS, but not significantly higher. Additionally, haplotypes and nucleotide diversities were slightly higher in the NZ pup collection $(h=0.753 \pm 0.028, \pi=0.004 \pm 0.002)$, than AUS ones $(h=0.713 \pm 0.038$, $\pi=0.001 \pm 0.001 ;$ Table 2.2). A rarefaction analysis showed that most of the populations (excluding Chatham Rise collections, $\mathrm{CHA}$ ) did not reach a plateau when the cumulative allelic richness was plotted against the sequences analysed (Figure 2.3). 
Table 2.2: Genetic variability from the control region sequences of Galeorhinus galeus for the open waters (OW) and pupping areas (PA). Sampling size $(N)$, number of segregation sites $(k)$, number of haplotypes $(n)$, private haplotypes $(p h)$, haplotype diversity $(h)$, and nucleotide diversity $(\pi)$.

\begin{tabular}{|c|c|c|c|c|c|c|c|c|c|}
\hline Sampling & Region & Sites & Date & $N$ & $k$ & $n$ & $p h$ & $h$ & $\pi$ \\
\hline & $\mathrm{NZ}$ & ENI & 2010 & 30 & 5 & 7 & 1 & $0.654 \pm 0.085$ & $0.001 \pm 0.001$ \\
\hline & & KAI & 2010 & 24 & 7 & 8 & 1 & $0.757 \pm 0.076$ & $0.001 \pm 0.001$ \\
\hline & & CHA & 2010 & 30 & 3 & 4 & 1 & $0.637 \pm 0.064$ & $0.001 \pm 0.001$ \\
\hline & & SOL & 2010 & 26 & 8 & 10 & 2 & $0.822 \pm 0.060$ & $0.002 \pm 0.001$ \\
\hline & & Total & & 110 & 8 & 15 & 5 & $0.735 \pm 0.032$ & $0.001 \pm 0.001$ \\
\hline \multirow[t]{12}{*}{ Open water } & AUS & SOU & 2007 & 47 & 8 & 10 & 3 & $0.770 \pm 0.040$ & $0.002 \pm 0.001$ \\
\hline & & POR & 2007 & 34 & 5 & 6 & 0 & $0.672 \pm 0.070$ & $0.001 \pm 0.002$ \\
\hline & & TAS & 2008 & 28 & 5 & 6 & 3 & $0.733 \pm 0.057$ & $0.002 \pm 0.001$ \\
\hline & & FRE & 2009 & 24 & 4 & 6 & 0 & $0.746 \pm 0.061$ & $0.001 \pm 0.001$ \\
\hline & & Total & & 133 & 8 & 15 & 6 & $0.729 \pm 0.027$ & $0.001 \pm 0.001$ \\
\hline & CHI & SAN & 2006 & 10 & 3 & 4 & 4 & $0.800 \pm 0.089$ & $0.002 \pm 0.001$ \\
\hline & NZ/AUS/CHI & Total & & 253 & 19 & 25 & 15 & $0.754 \pm 0.020$ & $0.002 \pm 0.001$ \\
\hline & $\mathrm{NZ}$ & $\mathrm{KAH}$ & 2009 & 11 & 2 & 3 & 0 & $0.473 \pm 0.162$ & $0.001 \pm 0.001$ \\
\hline & & MAN & 2009 & 21 & 5 & 6 & 1 & $0.752 \pm 0.061$ & $0.001 \pm 0.001$ \\
\hline & & NAP & 2009 & 33 & 8 & 8 & 2 & $0.686 \pm 0.063$ & $0.001 \pm 0.001$ \\
\hline & & KAP & 2009 & 19 & 6 & 8 & 3 & $0.871 \pm 0.044$ & $0.001 \pm 0.001$ \\
\hline & & MAP & 2009 & 30 & 8 & 9 & 3 & $0.789 \pm 0.065$ & $0.002 \pm 0.001$ \\
\hline \multirow[t]{8}{*}{ Puppping area } & & GOL & 2009 & 26 & 4 & 5 & 0 & $0.748 \pm 0.049$ & $0.001 \pm 0.001$ \\
\hline & & Total & & 140 & 8 & 17 & 9 & $0.753 \pm 0.028$ & $0.004 \pm 0.002$ \\
\hline & AUS & UPW & 2009 & 30 & 5 & 6 & 0 & $0.683 \pm 0.079$ & $0.001 \pm 0.001$ \\
\hline & & SWA & 1994 & 19 & 4 & 5 & 1 & $0.743 \pm 0.064$ & $0.001 \pm 0.001$ \\
\hline & & НOB & 1994 & 33 & 5 & 7 & 1 & $0.744 \pm 0.052$ & $0.001 \pm 0.001$ \\
\hline & & Total & & 82 & 5 & 10 & 2 & $0.713 \pm 0.038$ & $0.001 \pm 0.001$ \\
\hline & NZ/AUS & Total & & 222 & 13 & 21 & 4 & $0.738 \pm 0.023$ & $0.001 \pm 0.001$ \\
\hline & $\mathrm{TO}^{-}$ & & & 475 & 25 & 35 & 26 & $0.746 \pm 0.015$ & $0.001 \pm 0.001$ \\
\hline
\end{tabular}



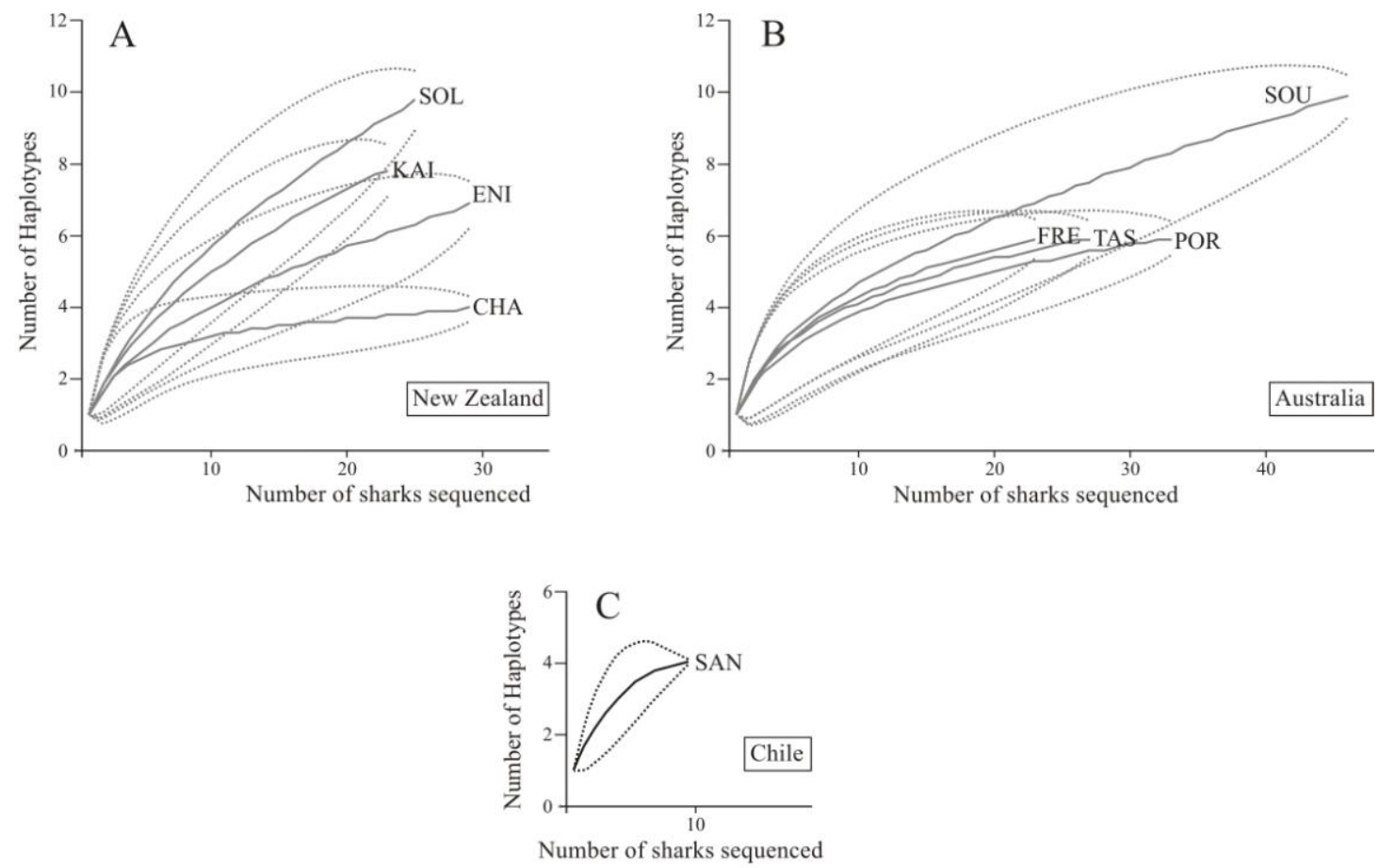

Figure 2.4: Haplotype rarefaction curve for the G. galeus CR sequences for each population sampled for the open waters sampling. Solid gray lines represent each population in A.New Zealand (ENI, KAI, CHA, SOL), B. Australia (SOU, POR, TAS, and FRE) and C. Chile. Dashed lines represent the $95 \%$ confidence intervals for rarefaction curves.

The hierarchical AMOVA analysis for the entire data set showed that the null hypothesis of global panmixia was rejected because of a significant level of genetic heterogeneity among collection sites $\left(\Phi_{S T}=0.37, p<0.001\right.$; Table 2.2A). Results of the temporal AMOVA revealed non-significant temporal genetic differentiation among year groups for NZ and AUS collections from 1994 to $2010\left(\Phi_{S T}=0.06, p=\right.$ 0.15). Significant spatial genetic differentiation was detected among regions i.e. NZ, AUS and CHI $\left(\Phi_{S T}=0.50, p<0.001\right)$. Subsequently, a second spatial AMOVA was run for NZ and AUS collections (excluding $\mathrm{CHI}$ ), which revealed non-significant differentiation across the Tasman Sea $\left(\Phi_{S T}=0.01, p=0.09\right)$. Therefore, the result of the second AMOVA suggested that statistical significance of the spatial pattern (among groups) of the first AMOVA was influenced by the CHI population, while the bulk of variation observed (>90\%, $p=0.10$ ) was explained within populations from New Zealand and Australia. This result was supported for six pairwise $\Phi_{S T}$ values, which showed weak genetic differentiation within New Zealand (e.g. ENI-SOL, $p=$ 0.002, Table 2.2B), and between New Zealand and Australia (e.g. SOL-POR, $p=$ 
0.007). However, they were non-significant after sequential Bonferroni correction of $\alpha$. All $\Phi_{S T}$ values between New Zealand and Australia comparisons were very low and ranged from -0.024 (SOU-FRE) to 0.120 (ENI-SOL), whereas they showed the highest and most significant $\Phi_{S T}$ values in comparison with the Chilean population, ranging from 0.188 (SOL-SAN) and 0.300 (CHA-SAN). The sequences from the pup collections revealed non-significant pairwise $\Phi_{S T}$ values among all populations in New Zealand and Australia (Table 2.2C). All comparisons that involved the KAH site were significantly different from the other nurseries sampled in NZ, but these genetic differences were most likely caused by the small sample size of KAH. Lastly, the Mantel test indicated there was no significant relationship between genetic distance and geographic distance for all sites across the Tasman Sea adult's collections $\left(R^{2}=\right.$ $0.04, p=0.38)$. 
Table 2.3: A. AMOVA among five temporal groups of G. galeus samples from Australasia region (NZ and AUS), among three spatial groups (NZ, AUS, and CHI), and two spatial groups (NZ and AUS).The d.f. is the degrees of freedom, SSD is the sum of squares, VC is variance components, and \% of total variation. B. Pairwise $\Phi_{S T}$ values (below diagonal) and $p$ values (above diagonal) between NZ, AUS and CHI populations from adult collections. The $p$ values are indicated between brackets for AMOVA, and significant $p$ values for the pairwise $\Phi_{S T}$ are represented by $*(\alpha=0.05), * *$ after sequential Bonferroni correction $(\alpha=0.0014)$. C. Pairwise $\Phi_{S T}$ values between NZ and AUS populations from pup collections. Significant $p$ values are represented by $*(\alpha=0.05)$.

\begin{tabular}{|c|c|c|c|c|c|c|}
\hline Structure tested & Source of variation & d.f & SSD & VC & $\%$ of total & $\Phi$-statictics \\
\hline \multirow[t]{3}{*}{ Global panmixia } & Among groups & 8 & 82.95 & 0.35 & 37.38 & $\Phi_{s t}=0.37(p<0.001)$ \\
\hline & Within populations & 244 & 144.09 & 0.59 & 62.62 & \\
\hline & Total & 252 & 227.04 & 0.94 & & \\
\hline \multirow{4}{*}{$\begin{array}{l}\text { Temporal } \\
(1994,2008,2009,2010)\end{array}$} & Among years & 3 & 3.39 & 0.01 & 1.37 & $\Phi_{S T}=0.06(p=0.15)$ \\
\hline & Among population within years & 6 & 3.42 & -0.01 & -0.12 & $\Phi_{c r}=0.01(p=0.07)$ \\
\hline & Within populations & 285 & 168.55 & 0.59 & 98.75 & $\Phi_{s c}=0.02(p=0.48)$ \\
\hline & Total & 294 & 175.36 & 0.60 & & \\
\hline \multirow[t]{4}{*}{ Spatial (NZ,AUS,CHI) } & Among regions & 2 & 77.89 & 0.57 & 48.59 & $\Phi_{s T}=0.50(p<0.001)$ \\
\hline & Among population within regions & 6 & 5.01 & 0.01 & 0.73 & $\Phi_{c r}=0.49(p=0.04)$ \\
\hline & Within populations & 244 & 144.09 & 0.59 & 50.69 & $\Phi_{s c}=0.01(p=0.09)$ \\
\hline & Total & 252 & 227.04 & 1.17 & & \\
\hline \multirow[t]{3}{*}{ Spatial (NZ and AUS) } & Among Tasman Sea (TS) & 1 & 0.84 & -0.01 & -0.02 & $\Phi_{s T}=0.01(p=0.09)$ \\
\hline & Among population within TS & 6 & 5.01 & 0.01 & 1.45 & $\Phi_{C r}=-0.01(p=0.52)$ \\
\hline & Within populations & 235 & 137.69 & 0.59 & 98.59 & $\Phi_{S C}=0.01(p=0.10)$ \\
\hline A & Total & 242 & 143.59 & 0.59 & & \\
\hline
\end{tabular}

B

\begin{tabular}{lccccccccc}
\hline \multicolumn{3}{c}{ New Zealand } & \multicolumn{9}{c}{ Australia } \\
\hline Sites & ENI & KAI & CHA & SOL & SOU & POR & TAS & FRE & SAN \\
\hline ENI & - & 0.395 & 0.298 & $0.002^{*}$ & $0.046^{*}$ & 0.544 & 0.170 & 0.125 & $p<0.001^{* *}$ \\
KAI & -0.001 & - & 0.633 & $0.048^{*}$ & 0.351 & 0.632 & 0.701 & 0.292 & $p<0.001^{* *}$ \\
CHA & 0.006 & -0.015 & - & $0.021^{*}$ & 0.293 & 0.788 & 0.736 & 0.305 & $p<0.001^{* *}$ \\
SOL & 0.120 & 0.050 & 0.078 & - & $0.032^{*}$ & $0.007^{*}$ & 0.102 & $0.034^{*}$ & $p<0.001^{* *}$ \\
SOU & 0.038 & 0.002 & 0.005 & 0.046 & - & 0.424 & 0.393 & 0.957 & $p<0.001^{* *}$ \\
POR & -0.008 & -0.014 & -0.021 & 0.090 & -0.002 & - & 0.489 & 0.594 & $p<0.001^{* *}$ \\
TAS & 0.019 & -0.018 & -0.020 & 0.031 & -0.001 & -0.007 & - & 0.314 & $p<0.001^{* *}$ \\
FRE & 0.030 & 0.009 & 0.007 & 0.061 & -0.024 & -0.013 & 0.006 & - & $p<0.001^{* *}$ \\
SAN & 0.290 & 0.225 & 0.299 & 0.188 & 0.218 & 0.278 & 0.239 & 0.231 & - \\
\hline
\end{tabular}

C

\begin{tabular}{lccccccc}
\hline Sites & KAH & MAN & NAP & KAP & MAP & GOL & UPW \\
\hline KAH & - & $0.034^{*}$ & 0.056 & $0.020^{*}$ & 0.111 & $0.029^{*}$ & 0.212 \\
MAN & 0.122 & - & 0.582 & 0.302 & 0.276 & 0.702 & 0.175 \\
NAP & 0.085 & -0.014 & - & 0.056 & 0.163 & 0.544 & 0.275 \\
KAP & 0.121 & 0.070 & 0.051 & - & 0.245 & 0.270 & 0.067 \\
MAP & 0.044 & 0.009 & 0.017 & 0.011 & - & 0.281 & 0.362 \\
GOL & 0.121 & -0.021 & -0.009 & 0.011 & 0.008 & - & 0.143 \\
UPW & 0.025 & 0.022 & 0.007 & 0.045 & -0.009 & 0.025 & - \\
\hline
\end{tabular}


The haplotype genealogy was constructed for the entire dataset (475 CR sequences), which showed evidence of two different clades of CR sequences from the entire dataset (NZ, AUS and CHI) separated by 5 mutational steps (Figure 2.4A). New Zealand and Australian haplotypes indicated a star-shaped genealogy dominated by three common haplotypes (haplotypes 1, 3 and 4). They differed by a single substitution and no haplotypes were more than 3 substitutions different from the most frequent haplotypes. Nineteen of the thirty haplotypes were unique for NZ or AUS populations $(\mathrm{NZ}=7, \mathrm{AUS}=12)$ and were found in a small number of $\mathrm{CR}$ sequences $(\leq 3)$. Eleven haplotypes were shared between NZ and AUS, corresponding to $36.7 \%$ from the overall number of haplotypes found in both regions. The examination of the distribution of the haplotype frequencies also revealed that there were three most common haplotypes (Hap1, 3 and 4). These haplotypes were distributed in different proportions in the majority of the sampling locations. Haplotype 1 was the principal haplotype found at all sampling locations, with the exception of SOL. The rest of the haplotypes were distributed in lower frequencies (Figure 2.4B).

Mismatch distribution for the entire dataset deviated significantly from the expected distribution under the constant and growth-decline population model (Figure $2.5 \mathrm{~A}$ ), which can be seen in the graph as a poor fit of the observed pairwise differences (solid line) and constant expected distribution model (dashed line). This result was supported by the lack of statistical significance of the Tajima's $D$ test ( $p=$ 0.13; Figure 2.5B). However, the growth-decline population model could not be rejected for NZ and AUS populations, when they were pooled together for the mismatch distribution (Figure 2.5A). Fu's $F_{s}$ values were negative and highly significant in NZ $(p=0.001)$, and in AUS $(p=0.001)$ suggesting population expansion in the past. This result is enhanced by the trend towards significance showed by the sum of squares distribution ( $S S D)$ from AUS $(p=0.05)$. In contrast, none of the $D$ values and $H_{r}$ index was statistically significant. Additionally, the resulting mismatch model was characterised by expansion time parameter $\tau=1.25$ in $\mathrm{NZ}$ and $\tau=1.33$ in AUS, and population sizes parameters $\theta_{0}=0$ and $\theta_{1}=99.999$ (Figure 2.5B). Using the mutation rates $(\mu)$ reported for CR and the estimation of $\tau$ by population, the time since the last expansion for NZ and AUS populations was estimated to be between $175 \mathrm{ka}$ to $346 \mathrm{ka}$ ago for the slower and faster mutation rates, respectively. Additionally, the BSPs indicated that NZ and AUS lineages for the CR 
of G. galeus showed a steady increase in the population size over $\sim 50$ or 75 ka ago using both mutation rates (Figure 2.6A,B). This result is similar to the results of $D$ and $H_{r}$ statistics, which did not show a genetic signature of sudden expansion through time. On the other hand, the BSPs for CHI showed that population size has been relatively stable over the last $20 \mathrm{ka}$ for both mutation rates (Figure 2.6C,D). A similar result was seen in the non-significance of the neutrality or demographic analysis and the ragged shaped of the mismatch distribution for $\mathrm{CHI}$ (Figure 2.5A).
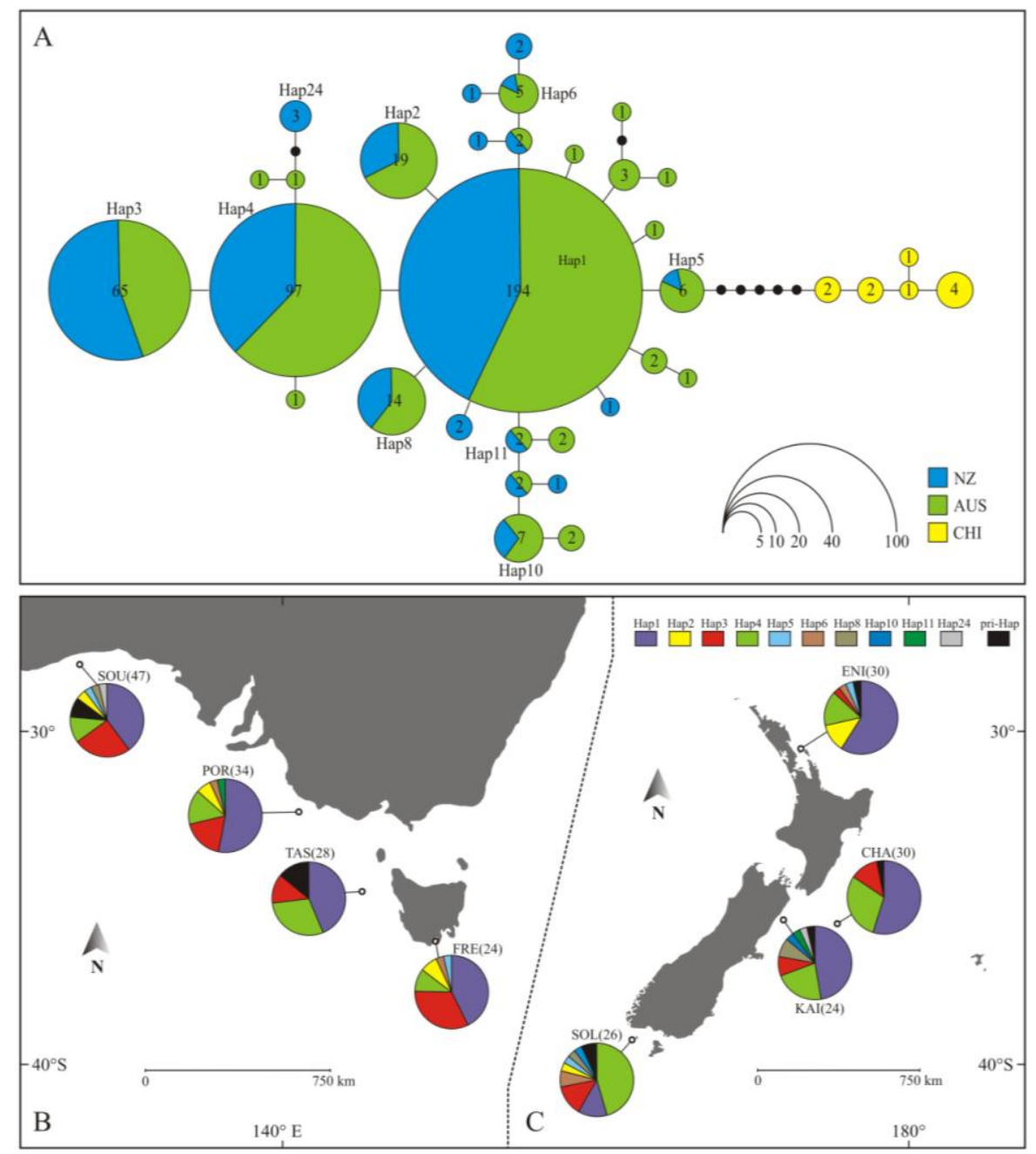

Figure 2.5: A. Haplotype genealogy for Galeorhinus galeus from maximum likelihood (ML) tree of $475 \mathrm{CR}$ sequences. Circles represent the haplotypes with area being equivalent to frequency. The number of individuals is reflected inside the circles. Each line indicates one mutational step between haplotypes and small black circles indicates hypothetical missing haplotypes. Colours represent geographic regions. B. Haplotype frequencies for the adult collection in New Zealand and Australia. The pie charts represent 10 different haplotypes (Hap1-10) and the black pie area reflects 11 private haplotypes pooled as one category called "pri-Hap". The number of private haplotypes by location is detailed in Table 2.1. Each colour indicates different haplotypes. 
Mismatch distribution plots

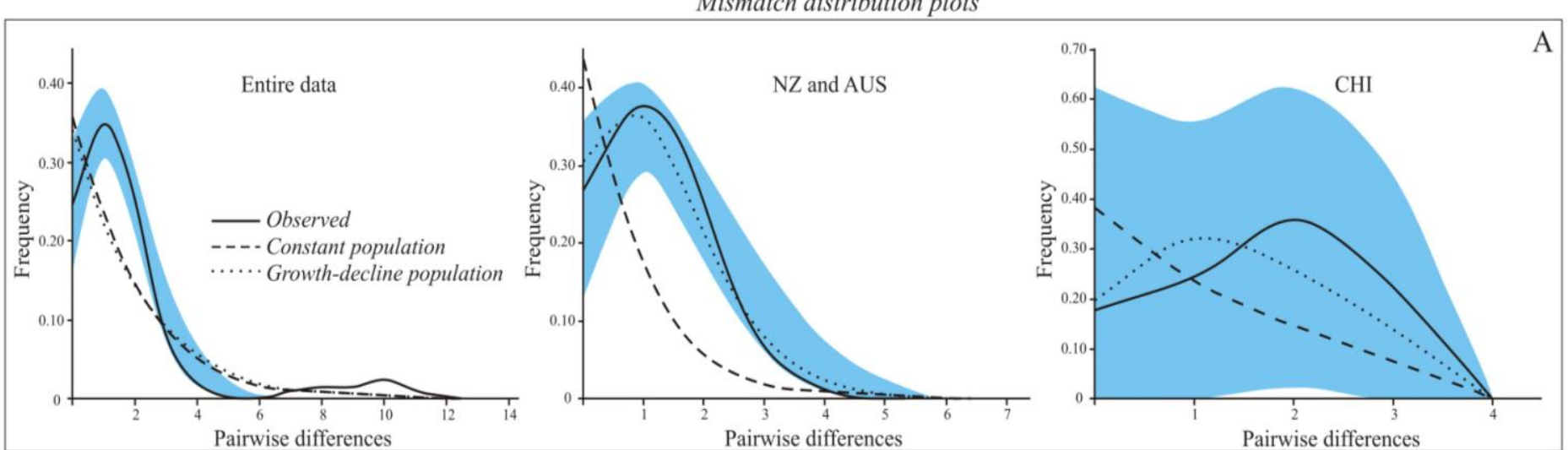

\begin{tabular}{|c|c|c|c|c|c|c|c|c|c|c|}
\hline \multirow[t]{2}{*}{ Site } & \multicolumn{2}{|l|}{ Neutrality } & \multicolumn{2}{|l|}{ Demographic } & \multirow[b]{2}{*}{$i$} & \multirow[b]{2}{*}{$\theta_{0}$} & \multirow[b]{2}{*}{$\theta_{l}$} & \multirow[b]{2}{*}{$t$} & \multirow[b]{2}{*}{$\theta_{S}$} & \multirow[b]{2}{*}{$N_{e f}$} \\
\hline & $D$ & $F_{s}$ & $H_{r}$ & SSD & & & & & & \\
\hline $\mathrm{NZ}$ & $-0.556(0.316)$ & $-8.870(0.001)$ & $0.079(0.082)$ & $0.004(0.179)$ & 1.252 & 0.007 & 99.999 & $175.000-326.000$ & 1.520 & $213.000-396.000$ \\
\hline AUS & $-0.649(0.290)$ & $-7.990(0.001)$ & $0.065(0.163)$ & $0.006(0.052)$ & 1.326 & 0 & 99.999 & $186.00-346.000$ & 1.650 & $231.000-430.000$ \\
\hline $\mathrm{CHI}$ & $1.227(0.880)$ & $-0.142(0.411)$ & $0.072(0.741)$ & $0.003(0.892)$ & 1.623 & 0 & 99.999 & $227.000-422.000$ & 1.060 & $148.200-276.000$ \\
\hline Entire data & $-1.090(0.134)$ & $-1.382(0.001)$ & $0.058(0.189)$ & $0.004(0.049)$ & 1.320 & NA & $\mathrm{NA}$ & NA & NA & $\mathrm{NA}$ \\
\hline
\end{tabular}

Figure 2.6: A. Comparison between observed and expected mismatch distributions of pairwise differences sequences of G. galeus under a constant and growth-decline population model performed from adult collection. Solid lines represent the observed pairwise differences, dashed lines the expected distribution for constant population model and dotted lines for growth-decline model. Blue areas encompass $95 \%$ C.I. for observed data. B. Table of neutrality tests, mismatch distribution, co-ancestry coefficient and effective population sizes estimates from CR sequences for three regional populations. The $p$ values are indicated between brackets for the statistics. Tajima test $(D)$, Fu's test $\left(F_{s}\right)$, Harpending's raggedness index $\left(H_{r}\right)$, sum of squared distribution $(S S D)$, population size before expansion $\left(\theta_{0}\right)$, population size after expansion $\left(\theta_{l}\right)$, age of population mutational time $(\tau)$, time since population expansion occurred $(t)$ for mutation rates of $4 \times 10^{-9}$ and $2.15 \times 10^{-9}$, respectively; coancestry coefficient $\left(\theta_{s}\right)$, and effective female population sizes for both mutation rates as well $\left(N_{e f}\right)$. 

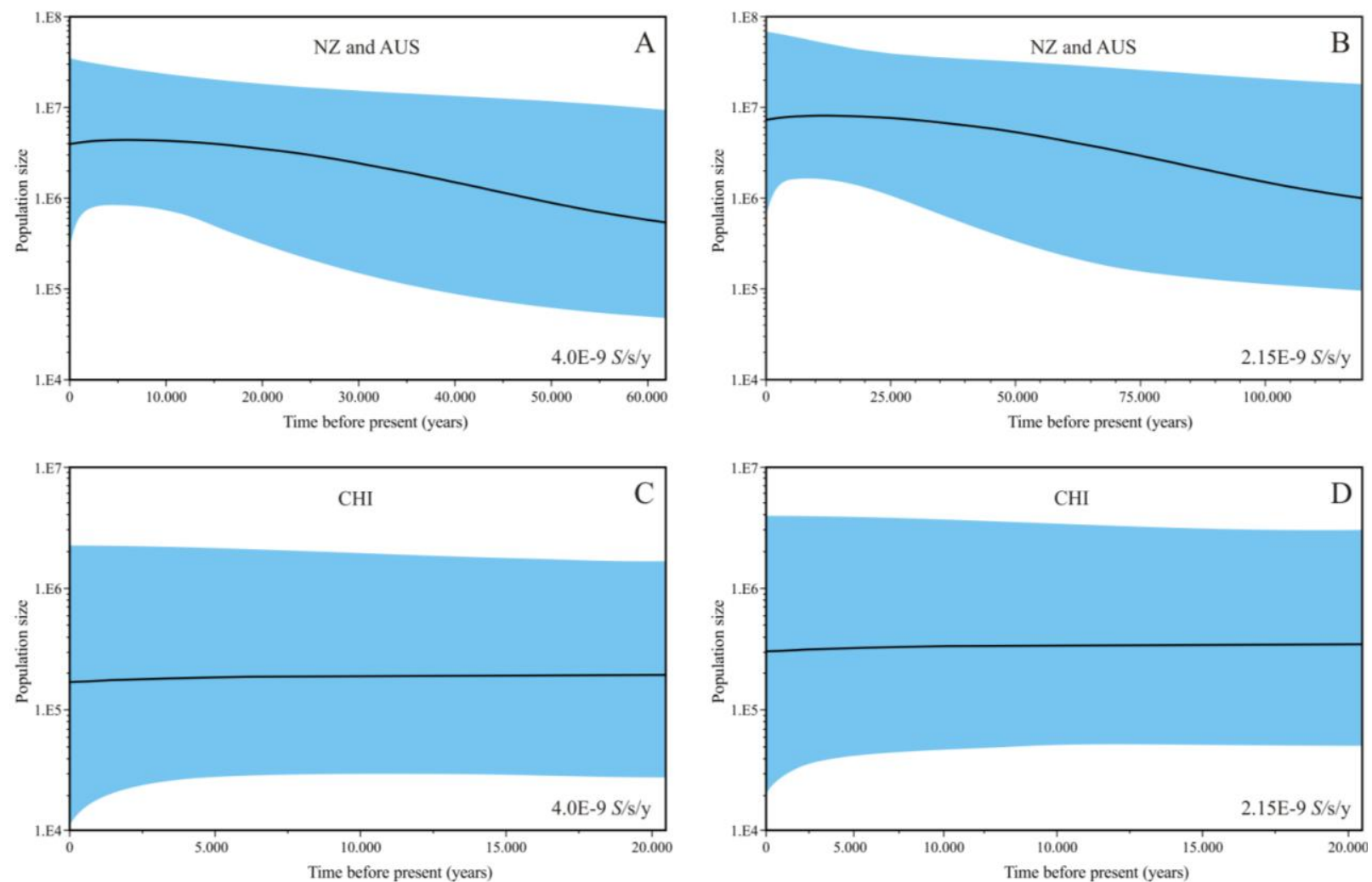

Figure 2.7: Bayesian skyline plots (BSPs) of G. galeus sequences represent population size changes through time (years) for NZ and AUS populations and CHI separated, inferred with CR lineages and assuming mutation rates of $4 \times 10^{-9}$ substitution/sites/year (Figure A and C) and $2.15 \times 10^{-9}$ substitution/sites/year (Figure B and D). Blue areas encompass 95\% highest posterior density (HPD) 


\subsubsection{Squalus griffini}

A 645bp sequence of the 5' end of the CR was determined for 145 of $S$. griffini. The base composition of this fragment was on average 2\% guanine, $34.4 \%$ adenine, $33 \%$ thymine, and $20.5 \%$ cytosine (33.5\% GC content). A comparison of sequences revealed 14 haplotypes, which were defined by 11 segregation sites that comprised 7 transitions and 4 transversions (GenBank Accession numbers KC431810-KC431823). The overall haplotype $(h)$ and nucleotide diversity $(\pi)$ were $0.695 \pm 0.023$ and $0.002 \pm 0.001$, respectively. Haplotype and nucleotide diversity were very similar for GIB, WES and KER sites $(\sim h=0.65, \pi=0.001)$, but there was less diversity in the CHA site $(h=0.49 \pm 0.058, \pi=0.001 \pm 0.001$; Table 2.4$)$. A rarefaction analysis revealed that most of the populations did not reach a plateau when the cumulate allelic richness was plotted against the sequences analysed (Figure 2.7).

Table 2.4: Genetic variability from the CR sequences for $S$. griffini. Sample size $(N)$, number of segregation sites $(k)$, number of haplotypes $(n)$, private haplotype $(p h)$, haplotypes diversity $(h)$, and nucleotide diversity $(\pi)$.

\begin{tabular}{lccccccl}
\hline Sites & Date & $N$ & $k$ & $n$ & $p h$ & $h$ & $\pi$ \\
\hline GIB & 2004 & 3 & 1 & 2 & 0 & $0.667 \pm 0.314$ & $0.001 \pm 0.001$ \\
WES & $2005-2009$ & 50 & 7 & 1 & 5 & $0.652 \pm 0.050$ & $0.002 \pm 0.001$ \\
CHA & $2007-2008$ & 54 & 4 & 5 & 2 & $0.486 \pm 0.058$ & $0.001 \pm 0.001$ \\
KER & $2007-2008$ & 38 & 8 & 7 & 4 & $0.627 \pm 0.076$ & $0.002 \pm 0.001$ \\
TOTAL & & 145 & 11 & 14 & 11 & $0.695 \pm 0.023$ & $0.002 \pm 0.001$ \\
\hline
\end{tabular}




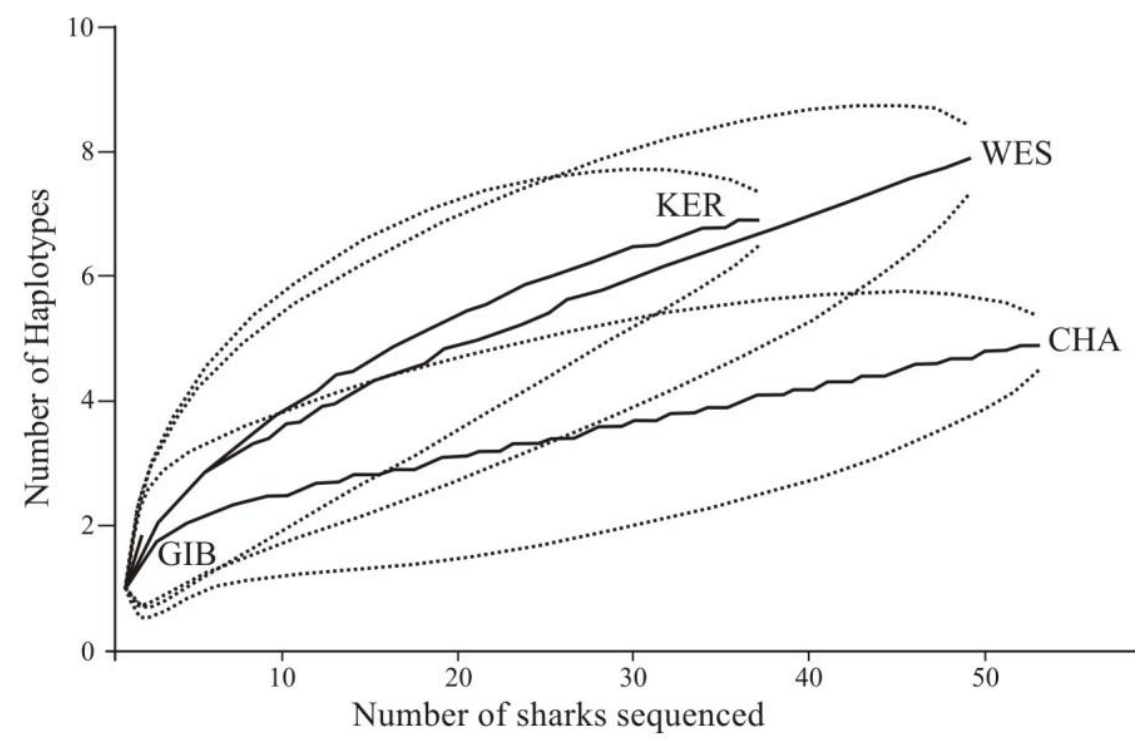

Figure 2.8: Haplotype rarefaction curve for the $S$. griffini $\mathrm{CR}$ sequences for each population sampled (GIB, WES, CHA, and KER). Dashed lines represent the 95\% confidence intervals for rarefaction curves.

The hierarchical AMOVA analysis for the entire data set showed that the null hypothesis of global panmixia was rejected due to significant level of genetic heterogeneity among collection sites $\left(\Phi_{S T}=0.32, p<0.001\right.$; Figure 2.8A). This results suggested genetic differentiation among regions for GIB-WES-CHA pooled and KER. These results were consistent with the pairwise $\Phi_{S T}$ values, which showed genetic differentiation between most comparisons that involved KER-WES and KERCHA ( $p>0.001$, Figure 2.8B). Conversely, there was non-genetic differentiation between KER-GIB ( $p=0.062)$ because of small sample size for GIB $(N=3)$, but it is clear the differences in haplotype distribution in comparison with KER (Figure 2.8D). The $\Phi_{S T}$ values varies between -0.064 (GIB-CHA) and 0.384 (WES-KER). Mantel test indicated there was no significant relationship between genetic distance and geographic distance for the four sites sampled $\left(R^{2}=0.46, p=0.29\right)$. 


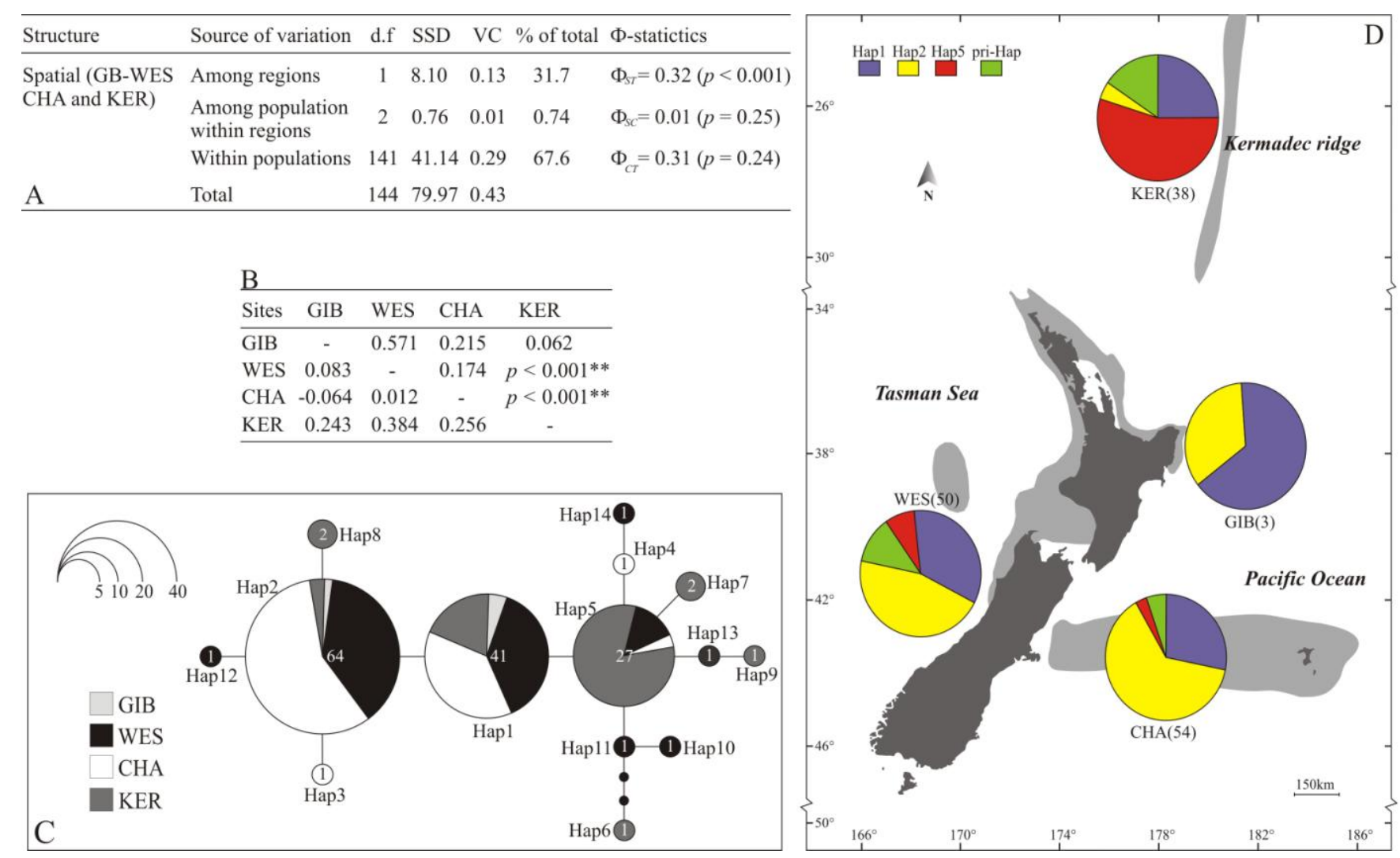

Figure 2.9: Population structure results for $S$. griffini from four sites: GIB, WES, CHA, and KER. A. Table of AMOVA results among two groups: first group was composed of GIB, WES, and CHA; and second was KER. The $p$ values are indicated between brackets for the statistics. The d.f. is the degrees of freedom, $S S D$ is the sum of squares, $\mathrm{VC}$ is variance components, and \% of total variation. B. Table of pairwise $\Phi_{S T}$ values (below diagonal) and $p$ values (above diagonal) between populations. The asterisks $(* *)$ represent significant values at $p<0.001 . \mathrm{C}$. Haplotype genealogy for $175 \mathrm{CR}$ sequences. Circles represent the haplotypes with area being equivalent to frequency. The number of individuals is reflected inside the circles. Each line indicates one mutational step between haplotypes and small black circles indicates hypothetical missing haplotype. D. Haplotype frequencies of CR sequences where the pie charts represent 3 different haplotypes (Hap1, 2 and 5) and the green pie area reflects 11 private haplotypes pooled as one category called "pri-Hap". 
The star-shaped haplotype genealogy showed evidence of population differentiation that indicate that KER samples have different haplotypes frequencies than the rest of the collection sites (Figure 2.8C). This results are consistent with the the pairwise $\Phi_{S T}$. Eleven of the fourteen haplotypes were unique to their collections site and they were found in a small number of CR sequences $(\leq 2)$, while the other three haplotypes were shared among all collection sites. The haplotypes typically differed by a single substitution when compared with the less frequent haplotypes ( $\leq$ 2). The private Hap 6 is separated by at least two mutational steps from all other haplotypes (Hap11). The distribution of haplotype frequencies of $\mathrm{CR}$ was characterised by two common haplotypes (Hap 1 and 2) that were found in GIB, WES, and CHA (Figure 2.8D). However, those haplotypes were found in a small number of sequences $(\leq 4)$ for KER, where haplotype 5 was the more frequently found.

Mismatch distribution for the entire dataset showed a good fit between the observed pairwise differences (solid line) and expected distribution model (dash line; Figure 2.9A). None of the $D$ values, $H_{r}$ and SSD values of population expansion were statistically significant. However, Fu's $F_{s}$ values showed a significant departure from neutrality for the entire data and when GIB-WES-CHA sequences were pooled together. Mismatch model was also characterised by expansion time parameter $\tau=$ 0.81 in GIB-WES-CHA and $\tau=0.94$ in KER, and population sizes parameters $\theta_{0}=0$ and $\theta_{1}=99.999$ (Figure 2.9B). Using the mutation rates $(\mu)$ reported for $\mathrm{CR}$ and the estimation of $\tau$ by population, the time since the last expansion was estimated between $157 \mathrm{ka}$ to $293 \mathrm{ka}$ years ago for the slower and faster mutation rates for GIB-WESCHA populations (Figure 2.9B). In addition, the BSPs result suggests that the GIBWES-CHA population experience a relatively steady population expansion in the past. The CR of for these three populations showed an increase in the population size over $\sim 30$ or 60 ka years ago according to both different mutation rates (Figure 2.10A,B). This result is strongly supporting for the results of $F_{s}$ values, which were able to detect genetic signature of sudden expansion through the time. However, the BSPs for KER indicated that the population size has been constant over the 70 or 125 ka years ago (Figure $2.10 \mathrm{C}, \mathrm{D})$. 
Mismatch distribution plots

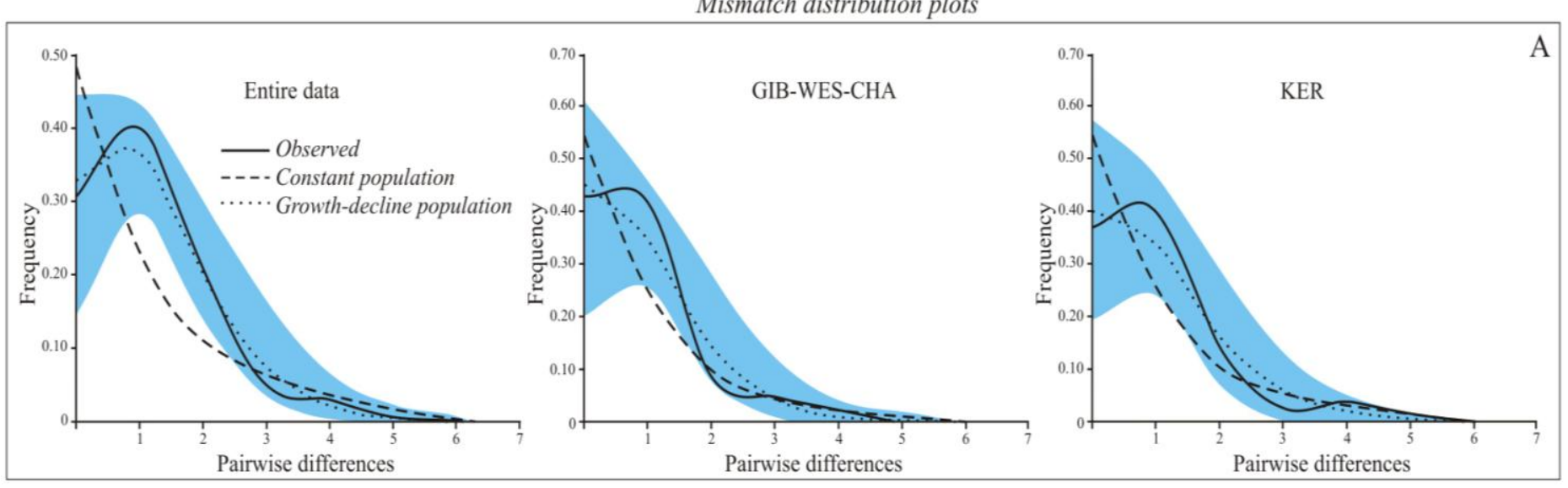

\begin{tabular}{|c|c|c|c|c|c|c|c|c|c|c|}
\hline \multirow[t]{2}{*}{ Sites } & \multirow{2}{*}{$\frac{\text { Neutrality tests }}{D}$} & \multicolumn{4}{|c|}{ Demographic analysis } & \multirow[b]{2}{*}{$\theta_{0}$} & \multirow[b]{2}{*}{$\theta_{l}$} & \multirow[b]{2}{*}{$t$} & \multirow[b]{2}{*}{$\theta_{S}$} & \multirow[b]{2}{*}{$N_{e f}$} \\
\hline & & $F_{s}$ & $H_{r}$ & SSD & $\tau$ & & & & & \\
\hline GIB-WES-CHA & $-0.890(0.207)$ & $-4.974(0.018)$ & $0.111(0.053)$ & $0.007(0.075)$ & 0.812 & 0 & 99.999 & $157.000-293.000$ & 1.563 & $302.000-562.000$ \\
\hline KER & $-1.371(0.075)$ & $-2.368(0.069)$ & $0.083(0.405)$ & $0.004(0.447)$ & 0.941 & 0 & 99.999 & $182.00-339.000$ & 1.904 & $368.000-685.000$ \\
\hline Entire data & $-1.091(0.131)$ & $-7.161(0.006)$ & $0.072(0.115)$ & $0.002(0.248)$ & 1.139 & NA & NA & NA & NA & NA \\
\hline
\end{tabular}

Figure 2.10: A. Comparison between observed and expected mismatch distributions of pairwise differences sequences of $S$. griffini under a constant and growth-decline population model. Solid lines represent the observed pairwise differences, dashed lines the expected distribution for constant population model and dotted lines for growth-decline model. Blue areas encompass $95 \%$ C.I. for observed data. B. Table of neutrality tests, mismatch distribution, coancestry coefficient and effective population sizes estimates from CR sequences for two regional populations: GIB-WES-CHA and KER. The $p$ values are indicated between brackets for the statistics. Tajima test $(D)$, Fu's test $\left(F_{s}\right)$, Harpending's raggedness index $\left(H_{r}\right)$, sum of squared distribution $(S S D)$, population size before expansion $\left(\theta_{0}\right)$, population size after expansion $\left(\theta_{1}\right)$, age of population mutational time $(\tau)$, time since population expansion occurred $(t)$ for mutation rates of $4 \times 10^{-9}$ and $2.15 \times 10^{-9}$, respectively; coancestry coefficient $\left(\theta_{s}\right)$, and effective female population sizes for both mutation rates as well $\left(N_{e f}\right)$. 

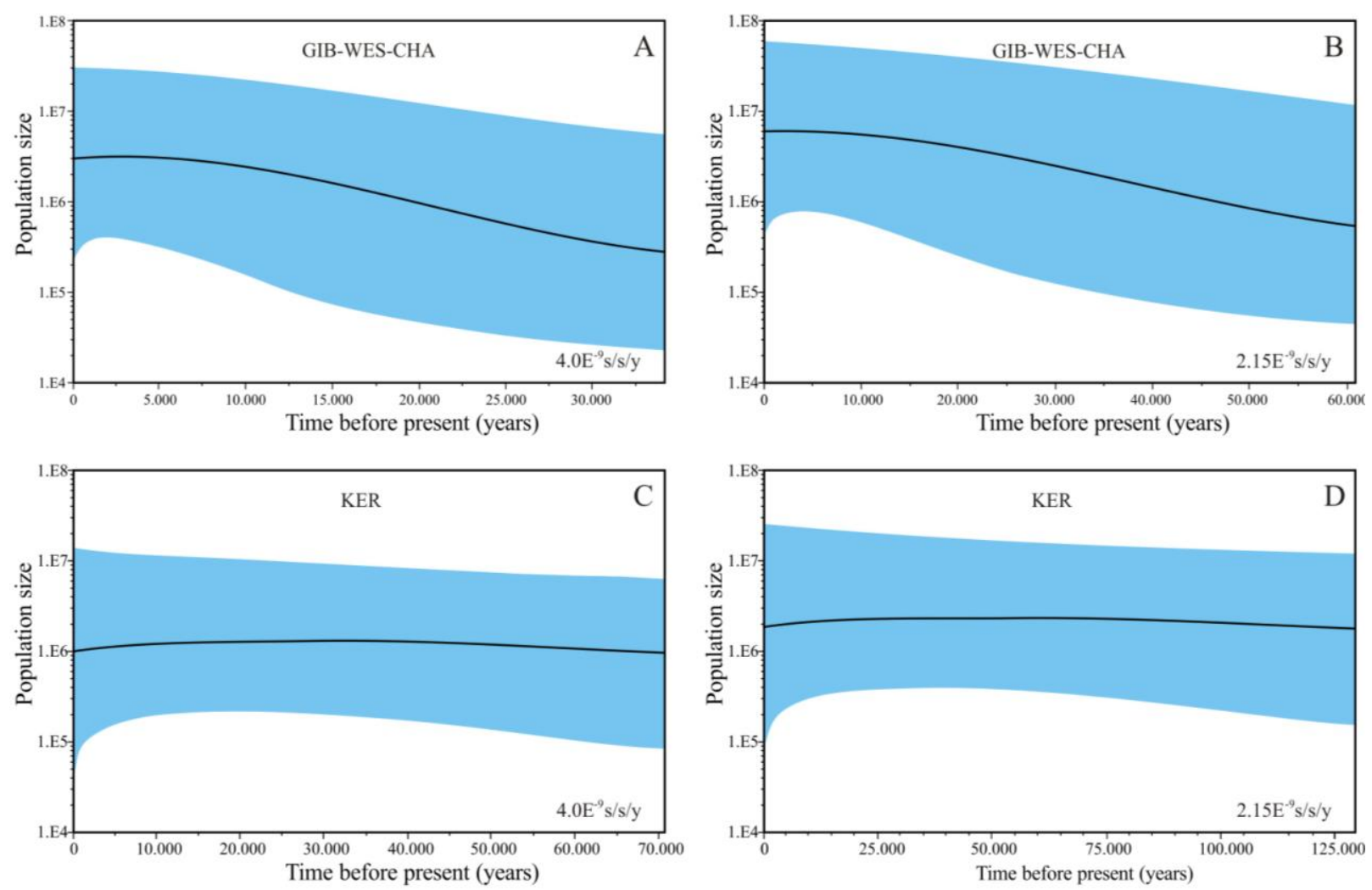

Figure 2.11: Bayesian skyline plots (BSPs) of S. griffini sequences represent population size changes through time (years) for three pooled populations (GIBWES-CHA) and KER inferred with CR lineages and assuming mutation rates of $4 \times 10^{-9}$ substitution/sites/year (Figure $\mathbf{A}$ and $\mathbf{C}$ ) and $2.15 \times 10^{-9}$ substitution/sites/year (Figure B and D). Blue areas encompass 95\% highest posterior density (HPD). 


\subsubsection{Sphyrna zygaena}

A $760 \mathrm{bp}$ sequence of the 5 ' end of the CR was determined using 156 of Sphyrna zygaena samples. The average base composition was $11.6 \%$ guanine, $31.2 \%$ adenine, $35.3 \%$ thymine, and $21.9 \%$ cytosine (32.3\% GC content). Comparison of sequences showed 14 haplotypes that were characterised by 5 segregation sites, comprising 3 transitions and 1 transversion (GenBank Accession numbers KC431824KC431828). The overall haplotype $(h)$ and nucleotide diversity $(\pi)$ were $0.615 \pm$ 0.024 and $0.002 \pm 0.001$, respectively. New Zealand and Australia showed the lowest haplotype and nucleotide diversity NZ and AUS (0), whereas Chile and Ecuador were more diverse (CHI: $h=0.434 \pm 0.082, \pi=0.001 \pm 0.001$; ECU: $h=0.568 \pm 0.058, \pi$ $=0.002 \pm 0.001$; Table 2.5). A rarefaction analysis for $\mathrm{CHI}$ and ECU populations indicate that cumulative allelic richness by the number of sequences analysed reached a plateau (Figure 2.11). Because NZ and AUS populations were characterised by only one haplotype for the $\mathrm{CR}$, the rarefaction curve was described as a flat line for both.

Table 2.5: Genetic variability from the CR sequences for $S$. zygaena. The sampling size $(N)$, number of segregation sites $(k)$, number of haplotypes $(n)$, private haplotypes $(p h)$, haplotypes diversity $(h)$, and nucleotide diversity $(\pi)$.

\begin{tabular}{lcccccll}
\hline Sites & Date & $N$ & $k$ & $n$ & $p h$ & $h$ & $\pi$ \\
\hline NZ & $2008-2009-2010$ & 49 & 0 & 1 & 0 & $0.000 \pm 0.000$ & $0.000 \pm 0.000$ \\
AUS & $2009-2010$ & 26 & 0 & 1 & 0 & $0.000 \pm 0.000$ & $0.000 \pm 0.000$ \\
CHI & $2006-2011$ & 46 & 4 & 4 & 1 & $0.434 \pm 0.082$ & $0.001 \pm 0.001$ \\
ECU & $2007-2008$ & 35 & 4 & 4 & 1 & $0.568 \pm 0.058$ & $0.002 \pm 0.001$ \\
TOTAL & & 156 & 5 & 5 & 2 & $0.615 \pm 0.024$ & $0.002 \pm 0.001$ \\
\hline
\end{tabular}




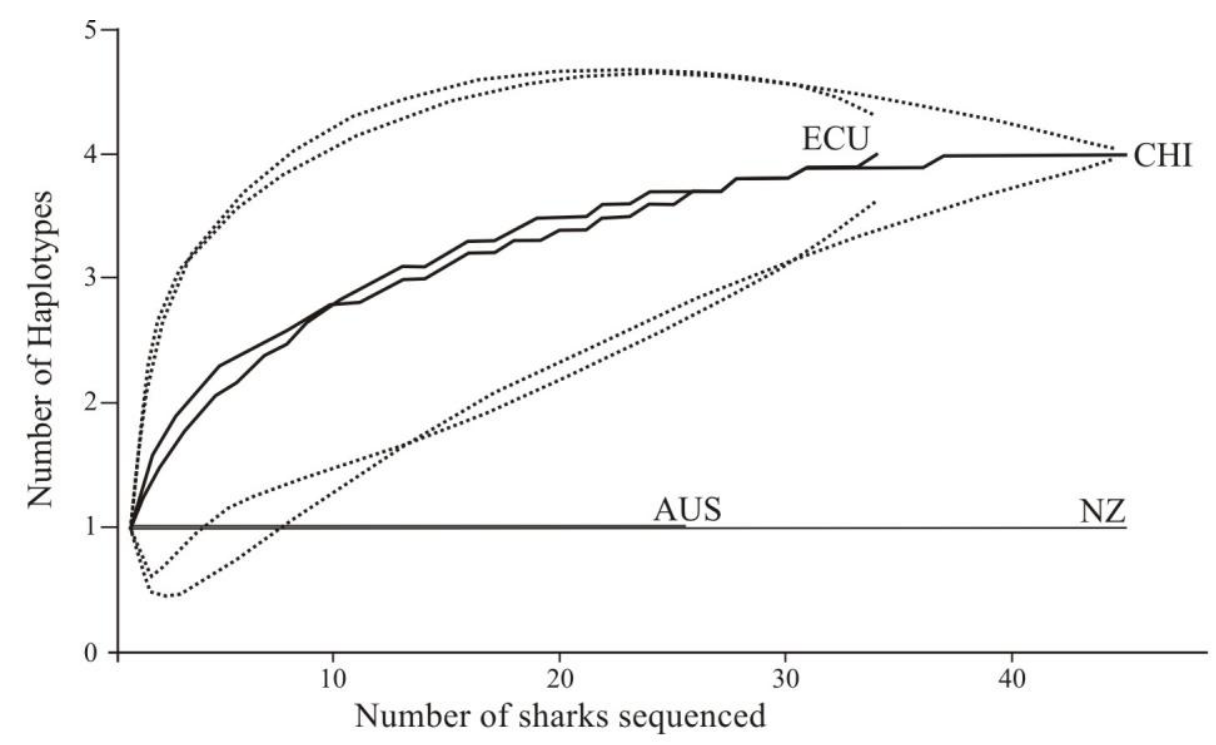

Figure 2.12: Haplotype rarefaction curve for the $S$. zygaena $\mathrm{CR}$ sequences for each population sampled for NZ, AUS, CHI and ECU. Dashed lines represent the 95\% confidence intervals for rarefaction curves.

The hierarchical AMOVA analysis for the entire data set showed that the null hypothesis of global panmixia was rejected due to a significant level of genetic heterogeneity among collection sites $\left(\Phi_{S T}=0.73, p<0.001\right.$; Figure 2.12A). This results suggested genetic differentiation among groups (NZ-AUS pooled) and (CHIECU). These results were consistent with the pairwise $\Phi_{S T}$ values, which indicated that NZ and AUS were genetically differentiated from CHI and ECU (Figure 2.12B). The $\Phi_{S T}$ values varied between 0 (NZ-CHI) and 0.741 (NZ-CHI). 


\section{A}

\begin{tabular}{llccccl}
\hline Structure tested & Source of variation & d.f & SSD & VC & $\%$ of total & $\Phi$-statictics \\
\hline $\begin{array}{l}\text { Spatial (NZ-AUS } \\
\text { and CHI-ECU) }\end{array}$ & Among regions & 1 & 26.83 & 0.34 & 71.86 & $\Phi_{S T}=0.73(p<0.001)$ \\
& $\begin{array}{l}\text { Among population } \\
\text { within regions }\end{array}$ & 2 & 0.67 & 0.01 & 1.18 & $\Phi_{C T}=0.71(p=0.33)$ \\
& Within populations & 152 & 19.42 & 0.13 & 26.96 & $\Phi_{S C}=0.04(p=0.07)$ \\
& Total & 155 & 46.97 & 0.47 & & \\
\hline
\end{tabular}

B

Sites NZ AUS CHI ECU

$\mathrm{NZ} \quad-0.999 p<0.001^{* *} p<0.001^{* *}$

AUS $0 \quad-p<0.001 * * \quad p<0.001 * *$

$\begin{array}{lllll}\mathrm{CHI} & 0.741 & 0.682 & - & 0.109\end{array}$

ECU $0.737 \quad 0.667$

$0.028 \quad-$

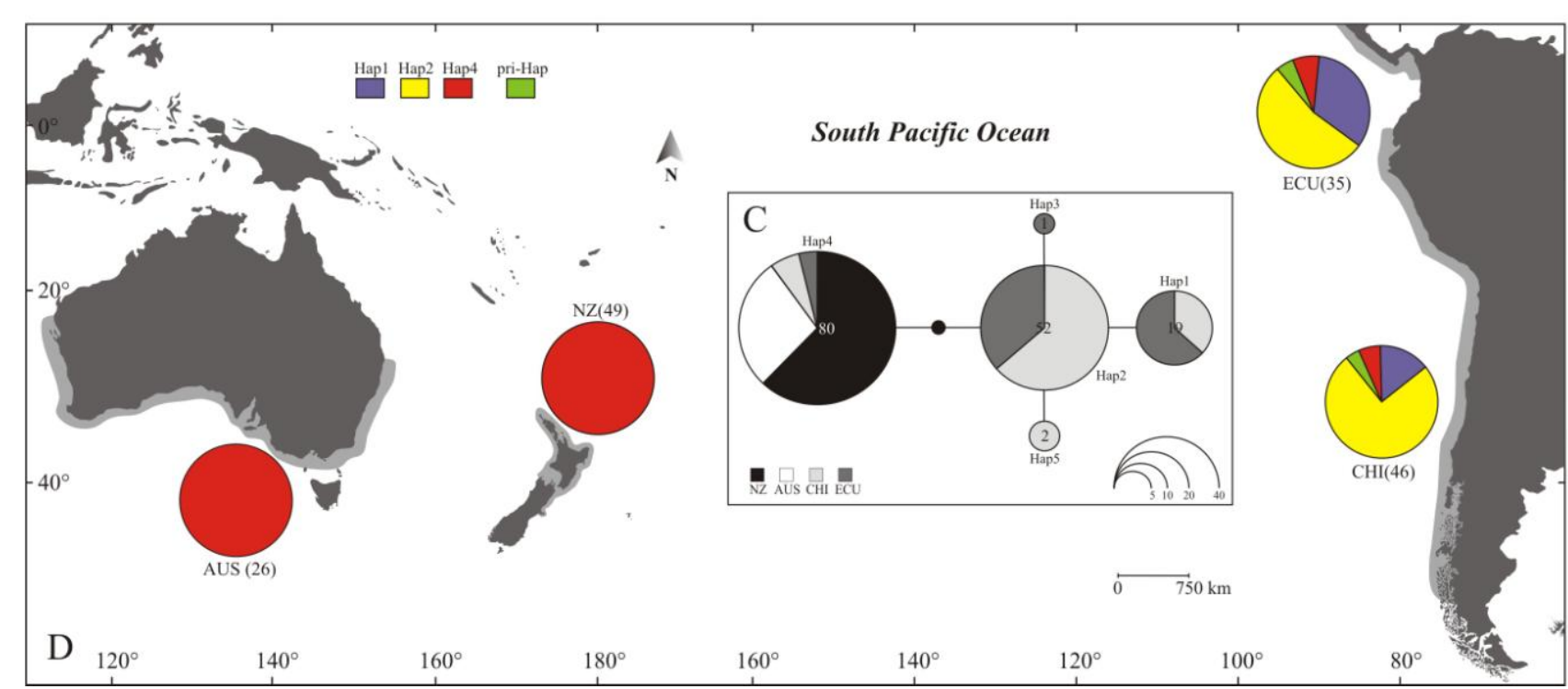

Figure 2.13: Population structure results for S. zygaena from four sites: NZ, AUS, CHI, and ECU. A. Table of AMOVA results among two groups: first group was composed of NZ and AUS, the second for CHI and ECU.The d.f. is the degrees of freedom, SSD is the sum of squares, VC is variance components, and \% of total variation. B. Table of pairwise $\Phi_{S T}$ values (below diagonal) and $p$ values (above diagonal) between populations. The asterisks (**) represent significant values at $p<0.001$. C. Haplotype genealogy for $156 \mathrm{CR}$ sequences. Circles represent the haplotypes with area being equivalent to frequency. The number of individuals is reflected inside the circles. Each line indicates one mutational step between haplotypes and small black circles indicates hypothetical missing haplotype. D. Haplotype frequencies of CR sequences where the pie charts represent different haplotypes (Hap1, 2 and 4) and the green pie chart reflect 2 private haplotypes pooled as one category called "pri-Hap". 
The haplotype genealogy showed evidence of population structure across the South Pacific Ocean based on the CR sequences (Figure 2.12C). Two of the five haplotypes were unique to sample sites and they were found at low frequency $(\leq 0.01)$ in $\mathrm{CHI}$ and ECU. The haplotypes from CHI differed from each other by a single nucleotide substitution. The distribution of haplotype frequencies of $\mathrm{CR}$ was characterised by a single haplotype for NZ and AUS (Hap 4), while CHI and ECU were represented by similar haplotype frequencies (Hap 1, 2, and 4) where the Hap 2 represented more than 50\% in both areas (Figure 2.12D), the rest was composed of Hap 1, 4 and two private haplotypes (one for $\mathrm{CHI}$ and another for ECU).

Mismatch distribution for the entire dataset and CHI-ECU deviated significantly from the constant expected distribution under the sudden expansion model, which are shown by the very poor fit of the observed pairwise differences (solid line) and expected distribution model (dashed line) (Figure 2.13A). This result was supported by the lack of statistical significance for both neutrality and demographic analysis (Figure 2.13B). Additionally, the resulting mismatch model was characterised by expansion time parameter $\tau=0.758$ for CHI-ECU and population sizes parameters $\theta_{0}=0$ and $\theta_{1}=99.999$ (Figure 2.13B). Using the mutation rates $(\mu)$ reported for $\mathrm{CR}$ and the estimation of $\tau$ by population, the time since the last expansion for CHI-ECU populations was estimated between $125 \mathrm{ka}$ to $232 \mathrm{ka}$ ago for the slower and faster mutation rates, respectively. Additionally, the BSPs indicate that CHI-ECU lineages for the CR of S. zygaena showed a stable population size over 60 or $125 \mathrm{ka}$ ago according to both different mutation rates (Figure 2.14). This result is strongly supported by the results of $D$ and $H_{r}$ statistics, which were not able to detect genetic signature of sudden expansion through the time. 
Mismatch distribution plots

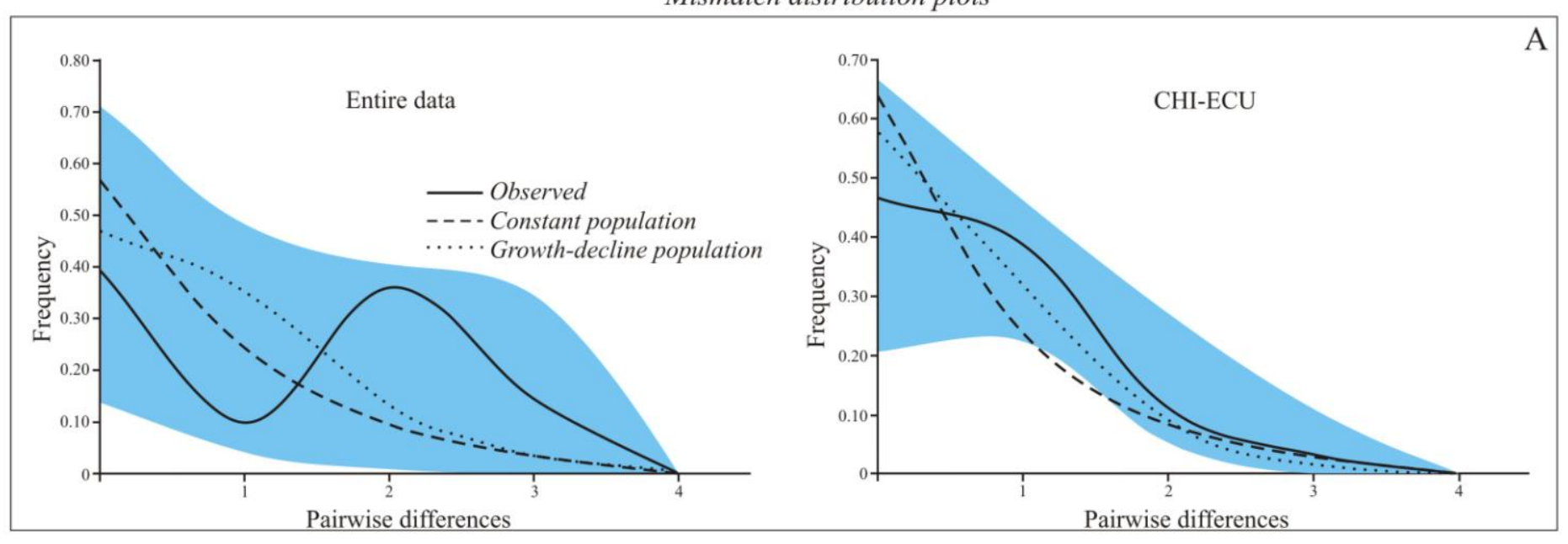

\begin{tabular}{|c|c|c|c|c|c|c|c|c|c|c|}
\hline \multirow[t]{2}{*}{ Sites } & \multicolumn{2}{|c|}{ Neutrality tests } & \multicolumn{8}{|c|}{ Demographic analysis } \\
\hline & $D$ & $F_{s}$ & $H_{r}$ & SSD & $\tau$ & $\theta_{0}$ & $\theta_{1}$ & $t$ & $\theta_{s}$ & $N_{e f}$ \\
\hline CHI-ECU & $-0.642(0.301)$ & $-1.598(0.201)$ & $0.090(0.392)$ & $0.002(0.474)$ & 0.758 & 0 & 99.999 & $125.000-232.000$ & 0.806 & $133.000-247.000$ \\
\hline Entire & $0.117(0.613)$ & $0.767(0.681)$ & $0.192(0.167)$ & $0.050(0.179)$ & 2.684 & NA & NA & NA & NA & NA \\
\hline
\end{tabular}

Figure 2.14: A. Comparison between observed and expected mismatch distributions of pairwise differences sequences of $S$. zygaena under a constant and growth-decline population. Solid lines represent the observed pairwise differences, dashes lines the expected distribution at constant population model and dot lines at growth-decline model. Blue areas encompass 95\% C.I. for growth-decline model. B. Table of neutrality tests, mismatch distribution, co-ancestry coefficient and effective population sizes estimates from CR sequences for CHI-ECU and Entire dataset. The $p$ values are indicated between brackets for the statistics. Tajima test $(D)$, Fu's test $\left(F_{s}\right)$, Harpending's raggedness index $\left(H_{r}\right)$, sum of squared distribution $(S S D)$, population size before expansion $\left(\theta_{0}\right)$, population size after expansion $\left(\theta_{l}\right)$, age of population mutational time $(\tau)$, time since population expansion occur $(t)$ for mutation rates of $4 \times 10^{-9}$ and $2.15 \mathrm{x}$ $10^{-9}$, respectively; coancestry coefficient $\left(\theta_{s}\right)$, and effective female population sizes for both mutation rates as well $\left(N_{e f}\right)$. 

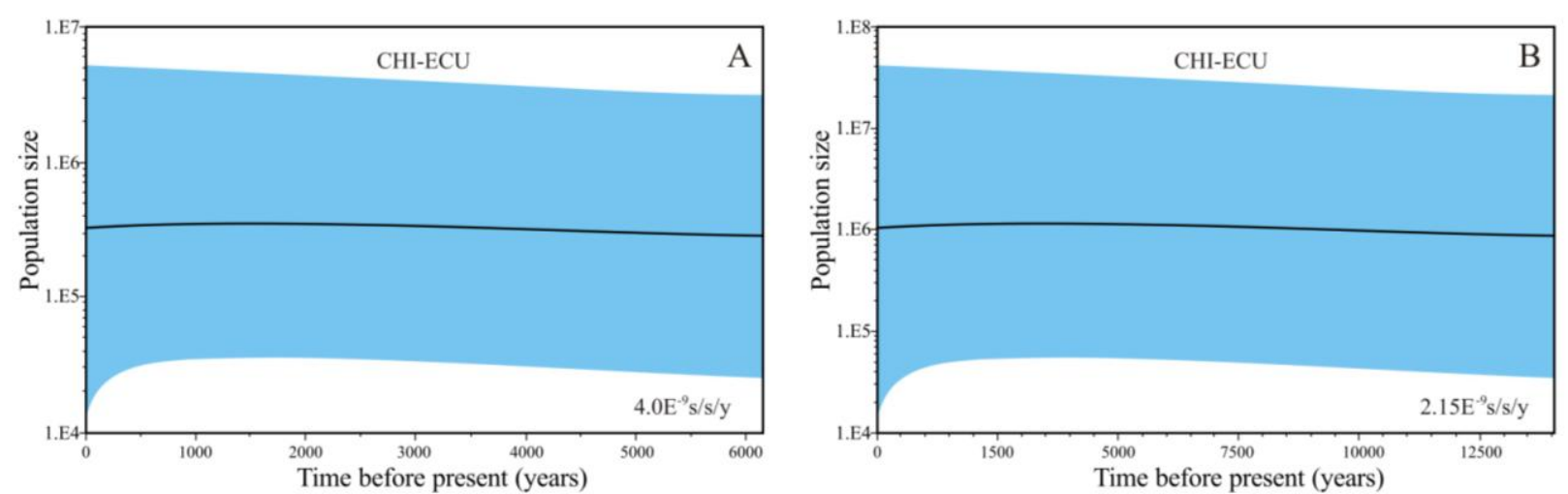

Figure 2.15: Bayesian skyline plots (BSPs) of S. zygaena sequences represent population size changes through time (years) for to pooled populations (CHIECU) populations inferred with CR lineages and assuming mutation rates for mutation rates of $4 \times 10-9$ substitution/sites/year (Figure A) and $2.15 \times 10-9$ substitution/sites/year (Figure B). Blue areas encompass 95\% highest posterior density (HPD). 


\subsection{Discussion}

\subsubsection{Genetic diversity}

Several mtDNA gene regions have been used to study genetic diversity and structure within and among populations of elasmobranch species. Most of the studies use the $\mathrm{CR}$ due to its higher level of polymorphism in comparison to other mtDNA genes. However, regions such as $c o I, n d 2, n d 4$, and $c y t b$ are also useful in molecular studies for providing a more detailed description of genetic diversity and different haplotype groups (Ward et al. 2007, Murray et al. 2008, Richards et al. 2009) or populations (Hauser 2009, Dudgeon et al. 2009, Ovenden et al. 2010, Schluessel et al. 2010, Veríssimo et al. 2010, Straube et al. 2011). Interestingly, most mtDNA gene regions are characterised by low haplotypic and nucleotide diversity, and only about $2 \%$ of the nucleotide positions are variable (Figure 2.1, 2.2 in Supplementary material, values were established across the sites studied). The mtDNA CR sequences from this study of G. galeus, S. griffini, and S. zygaena showed similar levels of variation to previous reports of CR sequences from G. galeus (Chabot \& Allen 2009) and S. zygaena (Testerman et al. 2008).

The level of intraspecific genetic diversity reported for shark species generally varies with habitat differences. Those species from pelagic and oceanic environments more commonly move long distances, and there is often an exchange of migrants between populations, which means the two populations function as a single large and biologically unit. The strength of drift is reduced and the mutations accumulated over time remain stable, resulting in high levels of genetic diversity e.g. Prionace glauca and Rhincodon typus (see Group III in Supplementary Figure 2.1 and Figure 6.1). Conversely, low levels of haplotypic and nucleotide diversity are found in coastal, reef, and near shore species e.g. Carcharhinus limbatus, Negaprion brevirostris. The findings of this chapter may reflect the fact that G. galeus is a species that occupies both coastal and oceanic habitats. The present study found relatively high levels of haplotype diversity within G. galeus populations $(h=0.75)$, when compared with the two other species ( $h=0.70, S$. griffini; and $h=0.62, S$. zygaena). The variation found in the G. galeus population was higher than the levels reported for other coastal species, including Carcharias taurus ( $h=0.72$, Ahonen et al. 2009), Carcharhinus leucas $(h=0.51$, Kark et al. 2011) and Negaprion acutidens $(h=0.28$, Schultz et al. 
2008). Conversely, larger ocean-going species such as Rhincodon typus (Castro et al. 2007, Ramírez-Marcías et al. 2007) and P. glauca (Ovenden et al. 2009) showed higher levels of mtDNA diversity than G. galeus.

\subsubsection{Genetic differentiation and population structure}

The dispersal capabilities, environmental conditions and life-history strategy are the major factors that shape genetic population structuring of marine fishes. Thus, continuously distributed species e.g. Thunnus albacares, Katsuwonus pelamis (Ely et al. 2005), Hoplostethus atlanticus (Varela et al. 2012), Isurus oxyrinchus (Schrey \& Heist 2003), Rhincodon typus (Castro et al. 2007), and Squalus acanthias (Veríssimo et al. 2010) displayed high level of genetic connectivity over large spatial scales (i.e. ocean basins). While, species such as Xiphias gladius (Rosel \& Block 1996), Makaira nigricans (Bluonaccorsi et al. 2001), and Prionace glauca (Ovenden et al. 2009) exhibits high level of genetic connectivity among populations. In contrast, less vagile species with discontinuous distribution e.g. Pomatomus saltatrix, Scromber japonicus (Graves 1998), Galeohrinus galeus (Chabot \& Allen 2009), and Sphyrna lewini (Duncan et al. 2006) shows comparatively greater intraspecific genetic differentiation across similar spatial scales. These findings have provided an important understanding of the levels of genetic connectivity in marine fishes i.e. bony fishes and shark species, and emphasize the use of genetics methods for understanding dispersal patterns.

One of the goals of this study was to examine the patterns of genetic population structuring of G. galeus, S. griffini and S. zygaena. Genetic connectivity was studied using CR sequence data over (a) large spatial scales across South Pacific Ocean (SPO) for G. galeus and S. zygaena, and (b) small spatial scales across Tasman Sea for G. galeus or within New Zealand waters for S. griffini, and along Eastern Pacific Ocean from Ecuador to Chile. At large spatial scales, the samples showed lack of genetic connectivity across SPO from either G. galeus or S. zygaena, suggesting that gene flow is highly restricted across SPO populations. These results are consistent with earlier genetic studies that used CR sequences data, which suggested that the Australian G. galeus samples were significantly different to South American (Chabot \& Allen 2009), and New Zealand and Australian S. zygaena samples showed similar 
patterns across SPO (Testerman et al. 2008). The biogeographic barrier between South America and Australasia is traditionally recognized as the "East Pacific Barrier (EPB)" (Dudgeon et al. 2012) and it extends approximately $7000 \mathrm{~km}$ along Pacific Ocean. The EPB affects population connectivity among several shark species, for example, Carcharhinus brachyurus (Benavides et al. 2011) showed a significant level of genetic differentiation between Australia/New Zealand and Peru. In contrast, species such as Squalus acanthias showed only a shallow, but statistically significant, level of genetic differentiation among collection site in New Zealand, Chile and Argentina (Veríssimo et al. 2010).

The haplotype diversity that was found in Chilean populations of G. galeus can be compare to the diversity reported from sites in Peru and Argentina by obtained the CR sequences from GenBank (Chabot \& Allen 2009). A comparison of South American sequences showed that there was a group of similar haplotypes in the region (Figure 2.3 Supplementary material). However, increasing the sample sizes and improving the distribution of sample coverage is needed to obtain a complete understand of the population structure of South American G. galeus. The samples from Chile and Peru shared two haplotypes, suggesting that there is gene flow along the continental shelf between these sites. Similar to G. galeus populations across the Tasman Sea, the shared genetic diversity of S. zygaena along Ecuador and Chile coasts indicates that there is genetic connectivity between these regions. Furthermore, S. lewini showed little genetic structuring among populations connected along the coastline, suggesting that genetic connectivity is often promoted by continuous continental margins (Duncan et al. 2006).

At a smaller spatial scale, several coastal species exhibit high levels of exchange of haplotypes in common, displaying lack of population structuring. The result of this study showed that both G. galeus and S. zygaena have a high level of genetic connectivity within and between New Zealand and Australia, and along Chile and Ecuador. These results are consistent with previous allozymes and RFLP-mtDNA markers studies that samples sites throughout the Tasman Sea and that found a weak, but not significant, level of genetic differentiation between sites on the east coast of New Zealand and western Tasman in Australia (Ward \& Gardner 1997). However, the conclusions that could be made from those types of genetic markers are limited because they typically had a low power of statistical resolution. The idea of a single 
panmictic G. galeus stock in Australasia region is strongly supported by the result of intensive tagging studies, which documented relatively important levels of migration across Tasman Sea (Olsen 1953, 1954, Stanley 1988, Paul 1988, Walker 1989, Walker et al. 1997, Francis \& Mulligan 1998, Hurst et al. 1999, Francis 2010). The lack of genetic differentiation throughout the Tasman Sea is correlated with major regional similarities in the life-history characters of G. galeus (e.g. size and age to reach sexual maturity), which suggests that the Tasman Sea (New Zealand or Australia) population has been evolving as a single evolutionary unit for a considerable period of time. Similar findings of a lack of genetic differentiation between New Zealand and Australia have been reported for other commercially important marine fishes, for example, the bony fish species Nemadactylus macropterus, Rexea solandri, Macruronus novaezelandiae, and Hoplostethus atlanticus (Ward \& Elliot 2001, Varela et al. 2011), and the for shark species Carcharhinus brachyurus and Carcharodon carcharias (Benavides et al. 2011, Pardini et al. 2001).

In contrast, $S$. griffini shows a low level of genetic connectivity within New Zealand waters, suggesting limited gene flow between sites at the Kermadec Islands and sites around the coast of New Zealand (see pairwise $\Phi_{S T}$ values). All the haplotypes were shared among four collection sites; however, the differences observed between sites were mainly driven by variation in the frequency of the most common haplotype (Hap5) at the Kermadec Islands. Similar patterns of variation of the common haplotype frequencies were found in Stegostoma fasciatum, which showed a level of genetic differentiation along Queensland coast in Australia (Dudgeon et al. 2009).

Sedentary behaviour, disjunct distributions and/or sex-biased dispersal are characteristics that are most often associated with high levels of genetic differentiation among large marine fish populations (Palumbi 1994). Sex-biased dispersal is when individuals of one sex tend to stay or return to their natal site or group of breeders (philopatric) while individuals of the other sex are more prone to disperse away from their natal area (Hueter et al. 2005). One approach used to detect philopatric behaviour is to test for difference between the patterns of genetic structure detected using biparental inherited nuclear genome (e.g. microsatellites) and the pattern detected using maternally transmitted mtDNA. Higher $F_{S T}$ values for mtDNA, but 
lower $F_{S T}$ values for nuclear markers are often taken to indicate a higher level of site fidelity in females than males (Hueter et al. 2005). Sex-specific patterns of population genetic variation have been observed in several species of sharks e.g. Carcharodon carcharias (Pardini et al. 2001), Isurus oxyrhinchus (Schrey \& Heist 2003), Negaprion brevirostris (Schultz et al. 2008), Carcharhinus lumbeus (Portnoy et al. 2010), Carcharhinus leucas (Karl et al. 2011). These studies demonstrated that females typically remained closer to their home territories, and males tend to roam over larger distances.

The use of biparentally inherited genetic markers e.g. microsatellite, single nucleotide polymorphic (SNPs), may provide more insight to understand whether genetic connectivity is reflecting the same dispersal pattern in both sexes or if there is a difference. Microsatellites are widely dispersed and its distribution cover independent regions along the chromosomes, with no known coding functions (unlike genes which code for specific proteins). The lack of coding constraints ensures that mutations accumulate more quickly than coding regions of the DNA. Future studies should consider (a) increasing the sample size and adding new collection sites, (b) employing nuclear markers such as microsatellites in conjunction with other mitochondrial markers (e.g. ND2 gene) to compare the pattern of population structure using different inheritance molecular markers, and (c) comparing genetic samples of newborn young and/or pups with microsatellite markers would be a valuable steps to understand particularly the genetic structuring of G. galeus.

\subsubsection{Demographic History}

Overfishing and habitat degradation are pressures that can reduce population size, which will increase the strength of genetic drift and cause genetic variation to be lost. The Tajima's $D$ and Fu's $F_{s}$ tests were used to investigate whether the DNA sequences conformed to a selectively neutral model of evolution, but these tests are also sensitive to changes in population size. The tests gave negative values for the populations data from the three shark species, which indicated that there was an excess of low frequency haplotypes. If it is assumed that natural selection has been a negligible force and the CR has been influenced primarily by genetic drift, then the Tajima's $D$ and Fu's $F_{s}$ results showed no genetic evidence for a declining population 
size. However, demographically the population could have recently shrunk but the process of drift will require more time before the expected decline in genetic diversity is detectable. The test results are expected under the model of population connectivity mainly for G. galeus and S. griffini because of the high number of private haplotypes found in these populations. The observation that some populations such as G. galeus POR and FRE from Australia and S. griffini GIB from New Zealand did not have private haplotypes could be because the sample sizes at these sites were too small to detect all of the variation.

New coalescent and non-coalescent methods have led to some significant advances in the field of population genetics, of particular note are the methods used to infer the demographic history of a population from gene sequences (Drummond et al. 2005). Coalescent methods such as Bayesian skyline plots (BSPs) often provide more resolution than non-coalescent methods (e.g. Tajima's $D$ and Fu's $F_{s}$ and mismatch distribution) because they can account for how drift, mutation and migration influence a gene genealogy (Felsenstein 1992, Pybus et al. 2000). In this study both the coalescent and non-coalescent methods showed that G. galeus populations had a pattern of population size contraction followed by a period of expansion. This pattern was particularly obvious in the samples collected from sites in New Zealand and Australia. A similar pattern of population size change was observed in the data collected from S. griffini sample sites in Gisborne, Westland, and Chatham Rise. The mismatch distribution analysis also suggested that the populations have undergone a recent expansion. However, the time that has elapsed since these population expansions is slightly different for both species. The mean of the mismatch distribution for G. galeus was further to the right the $y$-axis than $S$. griffini population. The mismatch analysis suggested that the time since population expansion occurred for G. galeus was similar than S. griffini (175-326 ka and 157 to $293 \mathrm{ka}$ ago, respectively). However, the BSPs estimate for the age since expansion suggested a more recent times $\sim 60$ or 125 ka years ago for G. galeus and 70 or 125 ka years ago for S. griffini.

The combination of high haplotype diversity, low nucleotide diversity and a star-shaped haplotype genealogy for the mtDNA data, suggests that G. galeus and $S$. griffini experienced a loss of genetic variation because of a population size reduction and then the population size began to recover, which meant more new mutations 
persisted in the population as drift weakened. This pattern of genetic diversity (high $h$ and low $\pi$ ) is very common in shark species and it has been reported for species such as S. lewini (Duncan et al. 2006), Carcharhinus limbatus (Kenney \& Heist 2006), Negaprion brevirostris (Schultz et al. 2008), Carcharhinus brachyurus (Benavides et al. 2011), Centroscymnus coelolepis (Veríssimo et al. 2010) (see Figure 2.1 and 2.2 in Supplementary material). When the relationship between CR haplotype diversity and nucleotide diversity of 20 shark species was compared, three groups of species with different combinations of small and large values of $h$ and $\pi$ were observed. The first group was made up of nine species with low levels of both $h$ and $\pi$ (e.g. Sphyrna

lewini, S. zygaena, Carcharhinus leucas), which might have been caused by reduction in population size or local extinctions, and found by recolonization (Grant \& Waples 2000). The second group was characterised by large values of $h$ and lower values of $\pi$, which is a pattern typically caused by a population expansion reducing the strength of drift and retaining more haplotypes when they arise (Rogers \& Harpending 1992, Grant \& Waples 2000). According to the present and previous CR studies (Chabot \& Allen 2009), G. galeus, S. griffini, and S. zygaena from Ecuador and Chile fall into this second group $(h>0.5,0.0002 \leq \pi \leq 0.06$ ), which is comprised of most of the sampled shark species (e.g. S. lewini, Carcharhinus limbatus, Carcharhinus leucas, Carcharhinus brachyurus, Carcharodon carcharias, and Cetorhinus maximus). The last group comprises species with a high $h$ and moderate high $\pi$, which is a pattern consistent with a population that has been large and stable over a long period of time. This group is characterised by large highly mobile species that occur in continuous distributions rather than group I and II, which are composed of smaller shark species with discontinuous distributions (Graves 1998, Karl et al. 2011). Only five species $S$. lewini, Squalus acanthias, Rhincodon typus, C. obscurus and Prionace glauca showed this latter pattern.

\subsubsection{Management implication}

Population genetic studies are an important component of the science that underpins fisheries management decisions. When genetic markers show that fishery populations are genetically subdivided, the best explanation is that the differences have arisen because of restricted gene flow. Consequently, a set of stocks can be identified and 
stock assessment models can be applied to each stock in conjunction with information about their recruitment, growth, mortality, etc. However, if genetic data suggest that a fishery only has one panmictic stock but there is other evidence to suggest that there might be two or more stocks then the genetic results should be looked at critically. A fishery could be comprised of multiple stocks, but the stocks have only appeared recently which means the pattern cannot be seen in the genetic data yet (need more time), or that the genetic markers did not have the level of resolution required to detect multiple stocks. In this study, the genetic data suggested that there were single stocks of G. galeus and S. zygaena that extended across the economic zones of New Zealand and Australia, and another stock along the continental margins for G. galeus from Peru and Chile and for S. zygaena from Ecuador and Chile. The tagging data is consistent with these suggested stock models and there is not any compelling evidence to contradict this conclusion.

New Zealand and Australian waters are important fishing grounds for many commercial marine fish e.g. G. galeus. Despite some evidence that the Tasman Sea can act as a barrier for gene flow for marine commercial fish, the majority of the species surveyed show a lack of any significant barrier to dispersal (Pardini et al. 2001, Ward \& Elliot 2001, Benavides et al. 2011, Varela et al. 2012). However, despite of the complex migration patterns and population structuring of G. galeus described by intensive tagging programs and biological background of G. galeus in Australasia region, it is a difficult species for stock assessment and management purposes.

Although the mtDNA CR could not reject the null hypothesis of a single stock of G. galeus between New Zealand and Australia, this species has been separated into locally (New Zealand and Australia) manageable single stocks due to: (a) previous molecular markers showed low resolution to test genetic differentiation, (b) tagging data showed low rates of movements from Australia to New Zealand, supporting the hypothesis of two stocks, and (c) for administrative and logistic reasons G. galeus is considered as separate stocks. This is more precautionary for G. galeus in Australia because if the assumption is made of one single stock, there is no reason to reduce the fishing rates in Australia (Waples et al. 2008). It is uncertain whether these regional management units are adequate for G. galeus; moreover when the stock is depleted in Australia, and the fishery is considered sustainable in New Zealand. Additionally, it is 
also concerning that the apparent sustainability of G. galeus biomass in New Zealand waters has been used to maintain of the high TAC levels through the last decade (>3000 t.). In Australian waters the historical nurseries areas have reduced and/or stopped to produce newborn young or pupping activity e.g. Port Phillip, Port Sorell, and St. Helen (Stevens \& West 1997), and most of the productive nurseries are in New Zealand waters. 


\subsection{Appendices}

Supplementary Table 2.1: Genetic diversity from the control region sequences among 20 shark species by each sampling locations reported to the date. Sampling locations, sampling size $(N)$, haplotypes diversity $(h)$, nucleotide diversity $(\pi)$, effective female population sizes $\left(N_{e f}\right)$ are detailed by each reference. ID is the reference number for each population in the supplementary figure.

\begin{tabular}{|c|c|c|c|c|c|c|c|}
\hline Species & Sampling locations & $N$ & $h$ & $\pi$ & $N_{f}$ & References & ID \\
\hline \multirow[t]{9}{*}{ Carcharhinus limbatus } & Bulls Bay, South Carolina & 34 & 0.37 & 0.0004 & $\mathrm{n} / \mathrm{a}$ & Keeney \& Heist 2006 & 26 \\
\hline & Georgia & 13 & 0.46 & 0.0004 & $\mathrm{n} / \mathrm{a}$ & & 31 \\
\hline & Pine Island, Sound Florida & 45 & 0.79 & 0.0012 & $\mathrm{n} / \mathrm{a}$ & & 77 \\
\hline & Terra Ceia Bay, Florida & 45 & 0.72 & 0.0011 & $\mathrm{n} / \mathrm{a}$ & & 63 \\
\hline & Yankeetown, Florida & 45 & 0.80 & 0.0013 & $\mathrm{n} / \mathrm{a}$ & & 80 \\
\hline & Padre Island, Texas & 49 & 0.81 & 0.0015 & $\mathrm{n} / \mathrm{a}$ & & 81 \\
\hline & Laguna Yalahau, Yucatan & 60 & 0.79 & 0.0029 & $\mathrm{n} / \mathrm{a}$. & & 76 \\
\hline & Belize city, Belize & 13 & 0.68 & 0.0007 & $\mathrm{n} / \mathrm{a}$ & & 58 \\
\hline & Dangriga, Belize & 19 & 0.53 & 0.0005 & $\mathrm{n} / \mathrm{a}$ & & 37 \\
\hline \multirow[t]{4}{*}{ C. leucas } & North Carolina & 17 & 0.68 & 0.0017 & $\mathrm{n} / \mathrm{a}$ & Karl et. al. 2011 & 56 \\
\hline & Gulf Coast of Florida & 13 & 0.77 & 0.0014 & $\mathrm{n} / \mathrm{a}$ & & 74 \\
\hline & Northern Gulf & 23 & 0.25 & 0.0009 & $\mathrm{n} / \mathrm{a}$ & & 18 \\
\hline & Brazil & 49 & 0.33 & 0.0006 & $\mathrm{n} / \mathrm{a}$ & & 21 \\
\hline \multirow[t]{8}{*}{ C. brachyurus } & Australia & 37 & 0.24 & 0.0003 & $\mathrm{n} / \mathrm{a}$ & Benavides et. al. 2011 & 17 \\
\hline & Brazil & 1 & 0 & 0 & $\mathrm{n} / \mathrm{a}$ & & 2 \\
\hline & Mexico & 1 & 0 & 0 & $\mathrm{n} / \mathrm{a}$ & & 3 \\
\hline & Namibia & 26 & 0.84 & 0.0020 & $\mathrm{n} / \mathrm{a}$ & & 83 \\
\hline & $\mathrm{NZ}$ & 15 & 0 & 0 & $\mathrm{n} / \mathrm{a}$ & & 4 \\
\hline & Peru & 13 & 0.76 & 0.0018 & $\mathrm{n} / \mathrm{a}$ & & 71 \\
\hline & South Africa & 26 & 0.70 & 0.0014 & $\mathrm{n} / \mathrm{a}$ & & 60 \\
\hline & Spain & 1 & 0 & 0 & $\mathrm{n} / \mathrm{a}$ & & 5 \\
\hline \multirow[t]{3}{*}{ C. obscurus } & Indonesia & 8 & 0.86 & 0.0104 & $\mathrm{n} / \mathrm{a}$ & Ovenden et. al. 2009 & 105 \\
\hline & West Australia & 13 & 0.28 & 0.0016 & $\mathrm{n} / \mathrm{a}$ & & 20 \\
\hline & East Australia & 7 & 0.67 & 0.0030 & $\mathrm{n} / \mathrm{a}$ & & 54 \\
\hline \multirow[t]{4}{*}{ C. sorrah } & Indonesia & 8 & 0.71 & 0.0027 & $\mathrm{n} / \mathrm{a}$ & Ovenden et. al. 2009 & 62 \\
\hline & West Australia & 8 & 0.25 & 0.0007 & $\mathrm{n} / \mathrm{a}$ & & 19 \\
\hline & East Australia & 13 & 0.78 & 0.0032 & $\mathrm{n} / \mathrm{a}$ & & 75 \\
\hline & Gulf of Carpentaria & 20 & 0.64 & 0.0054 & $\mathrm{n} / \mathrm{a}$ & & 106 \\
\hline \multirow[t]{9}{*}{ C. plumbeus } & Taiwan & 46 & 0.90 & 0.0021 & $\mathrm{n} / \mathrm{a}$ & Portnoy et. al. 2010 & 84 \\
\hline & Hawaii & 23 & 0.54 & 0.0016 & $\mathrm{n} / \mathrm{a}$ & & 41 \\
\hline & Eastern Australia & 43 & 0.54 & 0.0022 & $\mathrm{n} / \mathrm{a}$ & & 42 \\
\hline & Western Australia & 25 & 0.93 & 0.0022 & $\mathrm{n} / \mathrm{a}$ & & 87 \\
\hline & South Africa/Indian & 15 & 0.87 & 0.0047 & $\mathrm{n} / \mathrm{a}$ & & 97 \\
\hline & Gulf of Mexico & 23 & 0.95 & 0.0021 & $\mathrm{n} / \mathrm{a}$ & & 90 \\
\hline & Delaware Bay & 52 & 0.91 & 0.0021 & $\mathrm{n} / \mathrm{a}$ & & 85 \\
\hline & Chesapeake Bay & 55 & 0.93 & 0.0022 & $\mathrm{n} / \mathrm{a}$ & & 88 \\
\hline & Eastern shore of Virginia & 47 & 0.91 & 0.0020 & $\mathrm{n} / \mathrm{a}$ & & 86 \\
\hline \multirow[t]{4}{*}{ Negaprion brevirostris } & Pacific Mexico & 9 & 0 & 0 & $\mathrm{n} / \mathrm{a}$ & Schultz et. al. 2008 & 1 \\
\hline & Bahamas & 30 & 0.54 & 0.0008 & $1.4 \times 10^{4}$ & & 38 \\
\hline & Brazil & 30 & 0.34 & 0.0004 & $2.6 \times 10^{4}$ & & 25 \\
\hline & Guinea Bissau & 11 & 0.62 & 0.0010 & $1.3 \times 10^{4}$ & & 49 \\
\hline \multirow[t]{5}{*}{ N. acutidens } & Taiwan & 5 & 0 & 0 & $\mathrm{n} / \mathrm{a}$ & Schultz et. al. 2008 & 10 \\
\hline & French Polynesia & 16 & 0 & 0 & $\mathrm{n} / \mathrm{a}$ & & 11 \\
\hline & New Caledonia & 2 & 1.00 & 0.0019 & $\mathrm{n} / \mathrm{a}$ & & 91 \\
\hline & Eastern Australia & 10 & 0.33 & 0.0006 & $1.6 \times 10^{4}$ & & 22 \\
\hline & Western Australia & 25 & 0.60 & 0.0015 & $1.7 \times 10^{4}$ & & 47 \\
\hline \multirow[t]{4}{*}{ Sphyrna zygaena } & $\mathrm{NZ}$ & 49 & 0 & 0 & $\mathrm{n} / \mathrm{a}$ & This study & 12 \\
\hline & Australia & 26 & 0 & 0 & $\mathrm{n} / \mathrm{a}$ & & 13 \\
\hline & Chile & 46 & 0.50 & 0.0010 & $\mathrm{n} / \mathrm{a}$ & & 35 \\
\hline & Ecuador & 35 & 0.57 & 0.0010 & $\mathrm{n} / \mathrm{a}$ & & 45 \\
\hline \multirow[t]{3}{*}{ S. lewini } & Indonesia & 28 & 0.74 & 0.0111 & $\mathrm{n} / \mathrm{a}$ & Ovenden et. al. 2009 & 110 \\
\hline & West Australia & 4 & 0.50 & 0.0163 & $\mathrm{n} / \mathrm{a}$ & & 113 \\
\hline & East Australia & 15 & 0.59 & 0.0002 & $\mathrm{n} / \mathrm{a}$ & & 44 \\
\hline
\end{tabular}




\begin{tabular}{|c|c|c|c|c|c|c|c|}
\hline Species & Sampling locations & $N$ & $h$ & $\pi$ & $N_{f}$ & References & ID \\
\hline \multirow[t]{15}{*}{ Sphyrna lewini } & Baja, Pacific & 44 & 0.51 & 0.0009 & $3.8 \times 10^{6}-1.1 \times 10^{7}$ & Duncan et. al. 2006 & 36 \\
\hline & Pac Panama, Pacific & 8 & 0.75 & 0.0019 & $1 \times 10^{6}-3.1 \times 10^{7}$ & & 71 \\
\hline & Hawai, Pacific & 44 & 0.09 & 0.0002 & $550-1600$ & & 14 \\
\hline & Phillippines, Pacific & 15 & 0.67 & 0.0110 & $1.1 \times 10^{4}-3.2 \times 10^{4}$ & & 111 \\
\hline & Taiwan, Pacific & 20 & 0.70 & 0.0073 & $2.6 \times 10^{6}-7.8 \times 10^{6}$ & & 108 \\
\hline & East Australia, Pacific & 32 & 0.66 & 0.0057 & $1.2 \times 10^{4}-3.5 \times 10^{4}$ & & 107 \\
\hline & Thailand, Indian & 2 & 0 & 0 & $\mathrm{n} / \mathrm{a}$ & & 8 \\
\hline & West Australia, Indian & 26 & 0.68 & 0.0095 & $3.4 \times 10^{3}-1.1 \times 10^{4}$ & & 109 \\
\hline & Seychelles, Indian & 12 & 0.17 & 0.0012 & $8.1 \times 10^{3}-2.7 \times 10^{4}$ & & 16 \\
\hline & South Africa, Indian & 25 & 0.42 & 0.0046 & $9 \times 10^{3}-3 \times 10^{4}$ & & 30 \\
\hline & West Africa, Atlantic & 6 & 0.33 & 0.0006 & $1.5 \times 10^{5}-5 \times 10^{5}$ & & 24 \\
\hline & Brazil, Atlantic & 3 & 0.67 & 0.0024 & $\mathrm{n} / \mathrm{a}$ & & 55 \\
\hline & Atlantic Panama & 2 & 1.00 & 0.0037 & $\mathrm{n} / \mathrm{a}$ & & 92 \\
\hline & Gulf of Mexico, Atlantic & 16 & 0 & 0 & $\mathrm{n} / \mathrm{a}$ & & 9 \\
\hline & East Coast USA, Atlantic & 16 & 0.53 & 0.0010 & $1.8 \times 10^{7}-6 \times 10^{6}$ & & 40 \\
\hline \multirow[t]{5}{*}{ Galeorhinus galeus } & Africa & 16 & 0.70 & 0.0010 & 46.808 & Chabot \& Allen 2009 & 59 \\
\hline & Australia & 50 & 0.76 & 0.0013 & 83.221 & & 73 \\
\hline & California & 26 & 0.73 & 0.0019 & 89.545 & & 68 \\
\hline & Peru-Argentina & 12 & 0.93 & 0.0022 & 63.680 & & 89 \\
\hline & UK & 12 & 0.79 & 0.0014 & 61.718 & & 79 \\
\hline \multirow[t]{3}{*}{ G. galeus } & New Zealand (NZ) & 110 & 0.74 & 0.0013 & $2.13 \times 10^{5}-3.96 \times 10^{5}$ & This study & 66 \\
\hline & Australia & 133 & 0.73 & 0.0013 & $2.31 \times 10^{5}-4.3 \times 10^{6}$ & & 65 \\
\hline & Chile & 10 & 0.80 & 0.0016 & $1.48 \times 10^{5}-2.76 \times 10^{5}$ & & 82 \\
\hline \multirow[t]{5}{*}{ Ginglymostoma cirratum } & Brazilian Islands & 37 & 0.43 & 0.0004 & $\mathrm{n} / \mathrm{a}$ & Karl et. al. 2012 & 28 \\
\hline & Natal, Brazil & 15 & 0.56 & 0.0005 & $\mathrm{n} / \mathrm{a}$ & & 43 \\
\hline & Bimini, Bahamas & 21 & 0.43 & 0.0004 & $\mathrm{n} / \mathrm{a}$ & & 27 \\
\hline & Florida, USA & 37 & 0.16 & 0.0001 & $\mathrm{n} / \mathrm{a}$ & & 15 \\
\hline & Glover's reef, Belize & 33 & 0.49 & 0.0004 & $\mathrm{n} / \mathrm{a}$ & & 32 \\
\hline \multirow[t]{6}{*}{ Carcharias taurus } & Eastern Australia & 66 & 0 & 0 & $\mathrm{n} / \mathrm{a}$ & Ahonen et. al. 2009 & 6 \\
\hline & Western Australia & 24 & 0.43 & 0.0021 & $\mathrm{n} / \mathrm{a}$ & & 29 \\
\hline & South Africa & 77 & 0.61 & 0.0013 & $\mathrm{n} / \mathrm{a}$ & & 48 \\
\hline & Northwest Atlantic & 12 & 0.53 & 0.0009 & $\mathrm{n} / \mathrm{a}$ & & 39 \\
\hline & Japan & 9 & 0 & 0 & $\mathrm{n} / \mathrm{a}$ & & 7 \\
\hline & Brazil & 6 & 0.33 & 0.0006 & $\mathrm{n} / \mathrm{a}$ & & 23 \\
\hline Carcharodon carcharias & California & 59 & 0.79 & 0.0013 & $\mathrm{n} / \mathrm{a}$ & Jorgensen et. al. 2010 & 78 \\
\hline \multirow[t]{2}{*}{ Cetorhinus maximus } & Pacific & 34 & 0.73 & 0.0013 & $\mathrm{n} / \mathrm{a}$ & Hoelzel et. al. 2006 & 67 \\
\hline & Atlantic & 27 & 0.72 & 0.0014 & $\mathrm{n} / \mathrm{a}$ & & 64 \\
\hline \multirow[t]{4}{*}{ Prionace glauca } & Indonesia & 19 & 0.89 & 0.0070 & $\mathrm{n} / \mathrm{a}$ & Ovenden et. al. 2009 & 102 \\
\hline & Mid-north Pacific & 20 & 0.89 & 0.0072 & $\mathrm{n} / \mathrm{a}$ & & 103 \\
\hline & West Australia & 4 & 1.00 & 0.0088 & $\mathrm{n} / \mathrm{a}$ & & 99 \\
\hline & East Australia & 17 & 0.89 & 0.0078 & $\mathrm{n} / \mathrm{a}$ & & 104 \\
\hline Squalus acanthias & Global distribution & 44 & 0.97 & 0.0072 & $\mathrm{n} / \mathrm{a}$ & Hauser et. al. 2007 & 100 \\
\hline \multirow[t]{4}{*}{ S. griffini } & Gisborne, NZ & 3 & 0.67 & 0.0010 & $\mathrm{n} / \mathrm{a}$ & This study & 57 \\
\hline & Chatham Rise, NZ & 54 & 0.48 & 0.0009 & $\mathrm{n} / \mathrm{a}$ & & 33 \\
\hline & South Westland, NZ & 50 & 0.65 & 0.0016 & $\mathrm{n} / \mathrm{a}$ & & 53 \\
\hline & Kermadec, NZ & 38 & 0.63 & 0.0015 & $3.68 \times 10^{5}-6.85 \times 10^{5}$ & & 50 \\
\hline \multirow[t]{5}{*}{ Rhincodon typus } & Atlantic & 19 & 0.93 & 0.0070 & $\mathrm{n} / \mathrm{a}$ & Castro et. al. 2007 & 101 \\
\hline & Western Indian & 18 & 0.95 & 0.0050 & $\mathrm{n} / \mathrm{a}$ & & 95 \\
\hline & Eastern India & 12 & 0.91 & 0.0040 & $\mathrm{n} / \mathrm{a}$ & & 96 \\
\hline & Northwest Pacific & 12 & 1.00 & 0.0050 & $\mathrm{n} / \mathrm{a}$ & & 93 \\
\hline & Northeast Pacific & 8 & 0.96 & 0.0060 & $\mathrm{n} / \mathrm{a}$ & & 94 \\
\hline \multirow[t]{3}{*}{ R. typus } & Bahia los Angeles & 19 & 0.95 & 0.0200 & $\mathrm{n} / \mathrm{a}$ & Ramírez-Macías et. al. 2007 & 115 \\
\hline & Bahia de la Paz & 13 & 0.90 & 0.0200 & $\mathrm{n} / \mathrm{a}$ & & 114 \\
\hline & Banco Gorda & 4 & 0.50 & 0.0100 & $\mathrm{n} / \mathrm{a}$ & & 112 \\
\hline Rhizoprionodon lalandii & Brazil & 83 & 0.82 & 0.0048 & $\mathrm{n} / \mathrm{a}$ & Mendonça et. al. 2009 & 98 \\
\hline Stegostoma fasciatum & Indo-West-Pacific & 20 & 0.48 & 0.0009 & $\mathrm{n} / \mathrm{a}$ & Dudgeon et. al. 2009 & 34 \\
\hline \multirow[t]{6}{*}{ Centroscymmus coelolepis } & Ireland & 45 & 0.64 & 0.0019 & $\mathrm{n} / \mathrm{a}$ & Verissimo et. al. 2010 & 52 \\
\hline & Portugal & 41 & 0.57 & 0.0014 & $\mathrm{n} / \mathrm{a}$ & & 46 \\
\hline & Madeira & 17 & 0.74 & 0.0020 & $\mathrm{n} / \mathrm{a}$ & & 70 \\
\hline & Azore & 40 & 0.74 & 0.0022 & $\mathrm{n} / \mathrm{a}$ & & 69 \\
\hline & Mauritania & 46 & 0.63 & 0.0017 & $\mathrm{n} / \mathrm{a}$ & & 51 \\
\hline & South Africa & 22 & 0.69 & 0.0019 & $\mathrm{n} / \mathrm{a}$ & & 61 \\
\hline
\end{tabular}




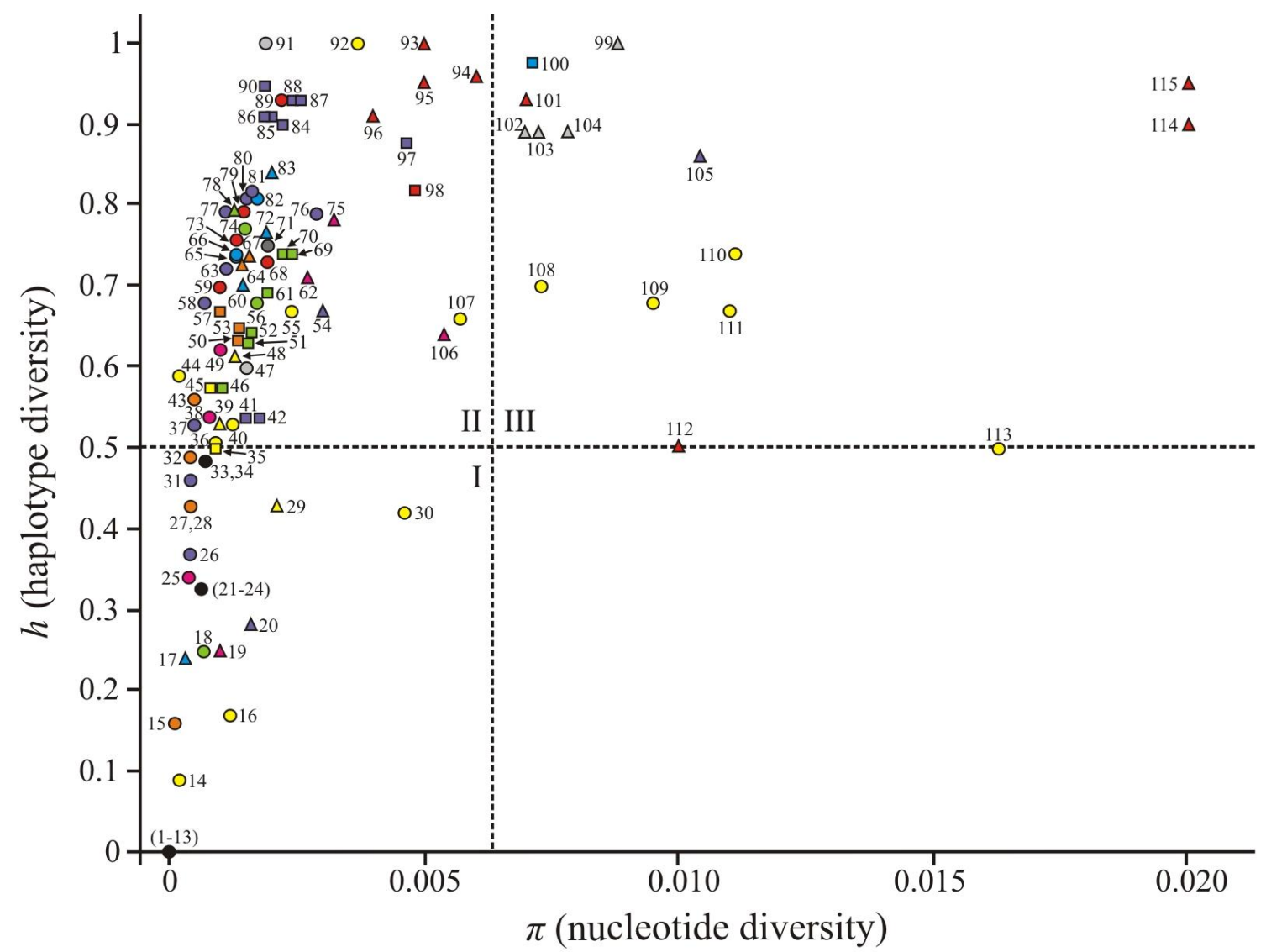

- Galeorhinus galeus (Chabot \& Allen 2009)

- Galeorhinus galeus (present study)

- Sphyrna lewini (Duncan et. al. 2006, Ovenden et. al. 2009)

- Ginglymostoma cirratum (Kark et. al. 2012)

- Carcharhinus leucas (Kark et. al. 2011)

- Carcharhinus limbatus (Kenney \& Heist 2006)

- Negaprion brevirostris (Schultz et. al. 2008)

- Negaprion acutidens (Schultz et. al. 2008)

$\Delta$ Rhincodon typus (Castro et. al. 2007, Ramírez-Marcías et. al. 2007)

$\Delta$ Carcharhinus brachyurus (Benavides et. al. 2011)

$\Delta$ Carcharhinus taurus (Ahonen et. al. 2009)

$\Delta$ Cetorhinus maximus (Hoelzel et. al. 2006)

$\Delta$ Carcharodon carcharias (Jorgensen et. al. 2010)

$\Delta$ Carcharhinus obscurus (Ovenden et. al. 2009)

$\Delta$ Carcharhinus sorrah (Ovenden et. al. 2009)

$\Delta$ Prionace glauca (Ovenden et. al. 2009)

- Rhizoprionodon lalandii (Mendonça et. al. 2009)

- Squalus acanthias (Hauser et. al. 2007)

$\square$ Sphyrna zygaena (present study)

口 Squalus griffini (present study)

口 Centroscymnus coelolepis (Verissimo et. al. 2010)

- Carcharhinus plumbeus (Portnoy et. al. 2010)

Supplementary Figure 2.1: Relationship between the haplotypes $(h)$ and nucleotide diversity $(\pi)$ of the CR sequences for 21 species of sharks. Only single values were coloured by species, however those species showing similar values were represented in black circles. 


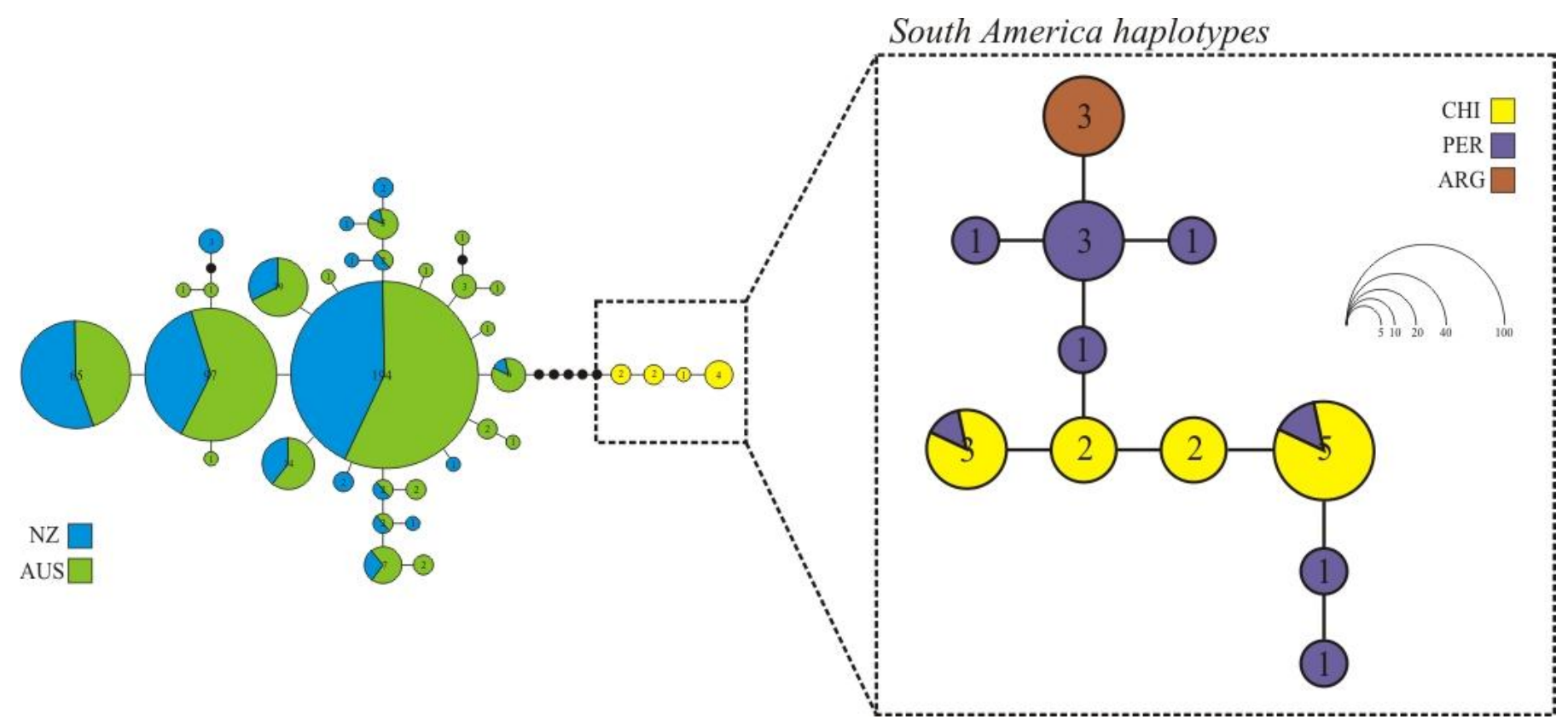

Supplementary Figure 2.2: Haplotype genealogy of G. galeus sequences from maximum likelihood (ML) tree of samples from present study (NZ, AUS, $\mathrm{CHI}$ ) and a haplotype network adapted according to the one reported by Chabot and Allen (2009) for CR sequences from Peru (PER) and Argentina (ARG). Circles represent the haplotypes with area being equivalent to frequency. The number of individuals is reflected inside the circles. Each line indicates one mutational step between haplotypes. Haplotypes from PER and ARG were obtained from GenBank. Refer to Figure 2.4A for details of NZ/AUS haplotypes geneology. 


\section{Chapter 3}

\section{The development of microsatellite DNA markers for school shark (Galeorhinus galeus)}

\subsection{Abstract}

School sharks (Galeorhinus galeus) are common in New Zealand and Australia, but there has only been a limited amount of research into their genetic structure. This chapter reports the development and testing of 15 G. galeus microsatellite loci that were developed using next-generation sequencing methods and fluorescent-labelled primer genotyping (ABI 3730). Approximately 200 G. galeus samples collected from three locations were genotyped and three of the microsatellite loci were found to be polymorphic (ranging from 2-9 alleles). The observed heterozygosity was between 0.135 and 0.510 and the expected heterozygosity between 0.169 and 0.598 , which was low when compared with most of elasmobranch species. The three polymorphic loci were be combined with another previously reported seven loci (see Chapter 4 and Chapter 5), and used to describe genetic diversity, population structuring, effective population sizes and parentage of G. galeus populations in Australasia. 


\subsection{Introduction}

DNA microsatellites are simple sequence repeats (SSRs), or highly repetitive DNA segments, of nuclear DNA. SSR often vary by the number of repetitive elements and can be highly variable within a population. SSR typically have a higher mutation rate than other areas of the nuclear genome because during replication the DNA polymerase enzyme can slip as it copies the repetitive element, which results in an insert or deletion event. Microsatellite alleles differ in size by multiples of the core repeat motif (2-6 bp) and they need to be distinguished using high resolution (O’Connell \& Wright 1997, Guichoux et al. 2011). DNA microsatellites are a popular genetic marker for determining levels of variation within and between populations and for studying parentage. This marker type has been widely used in fisheries for the identification of genetic stock structure, determining effective population size, species identification, product provenance and fisheries surveillance, and monitoring breeding patterns in aquaculture (Guichoux et al. 2011, Dichmont et al. 2011, Dudgeon et al. 2012).

The major disadvantage of microsatellite DNA is that development can be expensive and time-consuming. Traditionally there were two methodological approaches taken for their development, either hybridization selection (Armour et al. 1994), or linker and ligation procedures (Hamilton et al. 1999). However, the advent of next-generation sequencing (NGS) technology revolutionized the development process because this method can rapidly produce hundreds of thousands of nuclear DNA sequences that can be quickly searched for repeat regions and primers flanking these regions designed immediately. This approach also enables all repeat types to be discovered and is not limited by the number of DNA probes that are available in the laboratory when screening a genomic library.

The most common NGS approach uses the GS-FLX Titanium (Roche 454 Life Science, USA, commonly named as 454) platform because it is the best system for generating the large average sequence read sizes (approximately $500 \mathrm{bp}$ ) that are needed to provide a reasonable number of primer design options either side of the repeat element. Fluorescent dye labelled genotyping technology (e.g. ABI 3730) has enabled multiplexing and pool-plexing of SSRs markers, which is when several loci are combined and analysed together whenever their allele sizes do not overlap if they 
use that same coloured dye, but if the alleles sizes of loci do overlap then different coloured fluorescent dyes can be used to distinguish them (Schuelke 2000, Guichoux et al. 2011). This combined approach reduces the genotyping costs and analysis times.

The NGS approach can be described in four steps: (1) A sample of genomic DNA is first nebulized into small fragments. Each individual DNA strand is attached to a bead, and separated from all other strands into its own micro-PCR droplet (Emulsion PCR: a one tube oil-water mix that creates millions of separate droplets that contain all of the ingredients for micro-PCRs), then cycled to produce a large number of copies of each strand. The synthetic DNA sequences that form a cluster on each bead are read as light emitted during a pyrosequencing process. (2) The resultant DNA sequences are then searched and those containing a repetitive element are separated. (3) Those sequences initially selected are filtered. Each repeat-DNA type is identified and sequences that have repeats at the sequence ends (i.e. there is no room for a primer) are discarded. (4) The program Primer 3 is used to generate a range of primer options, the best primers (based on fragment size, small difference between primer melting temperatures, etc) are chosen, the oligonucleotides are synthesized and each locus is screened for polymorphism and whether they have technical problems (e.g. null alleles, ambiguities caused by stuttering, etc) (Abdelkrim et al. 2009, Guichoux et al. 2011).

The goal of this Chapter was to develop microsatellite markers for New Zealand and Australian G. galeus populations using the NGS technology (Roche 454). In Chapter 4 and 5 these new markers will be combined with previously published microsatellite markers and the panel of markers will be used to study the genetic variation of school sharks, describing the genetic diversity, assessing population genetic structuring, estimate population sizes and parentage study. 


\subsection{Methods}

DNA used for the development of the microsatellite library was isolated from five blood samples obtained from two juveniles of school shark captured in Manukau Harbour, New Zealand. Genomic DNA was purified using a standard phenolchloroform extraction protocol (Sambrook et al. 1989). The DNA quality and quantity was assessed using a NanoDrop spectrophotometer (model ND-1000) and visualised using agarose gel electrophoresis. The five best DNA extractions were sent to the Roche 454 sequencing service (Department of Anatomy and Structural Biology, Otago University). The DNA samples were prepared for the whole genome shotgun sequencing on the Roche Genome Sequencer FLX instrument utilizing the GS FLX Titanium Rapid Library Preparation Kit (Roche Applied Sciences, Indianapolis, USA) following the manufacturer's protocol. The library was quantified for DNA fragment size distribution and concentration (Agilent 2100 Bioanalyzer) and then processed with the GS FLX emulsion PCR. Sequencing was performed using 1/8th of an LR70 plate.

The resulting DNA sequence reads were converted into a single FASTA format file and screened for perfect and imperfect microsatellite motifs (di, tri, tetra) using the software QDD v1.0 (Meglécz et al. 2010). Primers for the identified microsatellite loci were designed using PRIMER3 (Rozen \& Skaletsky 2000), which was embedded in QDD software and the default settings were used. In addition, MSATCOMMANDER v0.8.1 (Faircloth 2008) was used to identify the number of perfect microsatellites within the FASTA file. Fifteen loci were chosen and their primer pairs were synthesized. An M13F sequence (5'TGTAAAACGACGGCCAGT) was added to the 5'-end of the forward primer so that a fluorescent label could be attached to the amplicons during the PCR process (for details about the M13 tailing method see Schuelke 2000). The primer sequences and PCR conditions for each locus are listed in Table 3.1.

Initially DNA isolated from between 5 and 15 samples from five different collection sites (four from New Zealand and one from Australia) were used to test for polymorphism and optimise the amplification of loci. Subsequently, 200 samples from New Zealand, Australia and Chile were screened using the markers to provide a more detailed characterization of each locus. Each microsatellite locus was PCR 
amplified separately in $10 \mu \mathrm{l}$ volumes containing $67 \mathrm{mM}$ Tris- $\mathrm{HCl} \mathrm{pH} 8.8,16 \mathrm{mM}$ $\left(\mathrm{NH}_{4}\right)_{2} \mathrm{SO}_{4}, 2.0 \mathrm{mM} \mathrm{MgCl}, 0.05 \mu \mathrm{M}$ of forward primer with an M-13 tail, $0.2 \mu \mathrm{M}$ of reverse primer, $0.05 \mu \mathrm{M}$ of a fluorescently labelled M-13 primer (either FAM, VIC, or PET, Invitrogen), $0.8 \mathrm{mM}$ of each dNTP, 0.3 units of BIOTAQ ${ }^{\mathrm{TM}}$ DNA Polymerase (Bioline), and $1 \mu 1$ of template DNA (50-80 ng/ $\mu 1$ DNA concentration). Thermal cycling was performed on a TGradient Thermal Block (Biometra, Goettingen, Germany). There was an initial denaturing step of $95^{\circ} \mathrm{C}$ for $5 \mathrm{~min}$, followed by 30 cycles of $94^{\circ} \mathrm{C}$ for $30 \mathrm{~s}, 62^{\circ} \mathrm{C}$ for $45 \mathrm{~s}$, and $72^{\circ} \mathrm{C} 45 \mathrm{~s}$, and finally a series of 8 cycles of $94^{\circ} \mathrm{C}$ for $30 \mathrm{~s}, 53^{\circ} \mathrm{C}$ for $45 \mathrm{~s}$, and $72^{\circ} \mathrm{C}$ for $45 \mathrm{~s}$ and a final extension of $72^{\circ} \mathrm{C}$ for $10 \mathrm{~min}$. Multiple loci were pool-plexed into wells on a 96-well format plate based on the combinations of loci pairs recommended by an analysis conducted using MULTIPLEX MANAGER 1.0 program (Holleley \& Geerts 2009). Capillary separation of the fluorescently labelled PCR-fragments was conducted on an ABI3730 Genetic Analyser (Massey University Genome Service). Allele sizes were determined using an internal lane standard LIZ 500 (ABI) and the Peak Scanner v1.0 software (Applied Biosystems).

The dataset was initially checked for the presence of null alleles, allele size shifts and scoring errors due to stuttering, using MICRO-CHECKER 2.2.3 (van Oosterhout et al. 2004). Genetic diversity estimates, such as the number of alleles per locus, observed $\left(H_{\mathrm{o}}\right)$, and expected $\left(H_{\mathrm{e}}\right)$ heterozygosities, tests for linkage disequilibrium (LD) between pairs of loci and deviation from Hardy Weinberg equilibrium (HWE), were calculated using ARLEQUIN 3.1.1 (Excoffier et al. 2005). The coefficient of inbreeding $\left(F_{\text {IS }}\right)$ was estimated using GENEPOP 4.0 (Raymond \& Rousset 1995). HWE significance was calculated using the exact test (Guo \& Thompson, 1992) with a Markov chain of $10^{4}$ steps and dememorization of $10^{4}$ steps.

\subsection{Results}

A total of 46,602 DNA sequences were generated by the FLX instrument. The length of the reads ranged from $62 \mathrm{bp}$ to $790 \mathrm{bp}$ with a mean of $246.3 \mathrm{bp} \pm 40.7 \mathrm{bp}$ (Figure 3.2). Overall, there were not many DNA sequences retrieved (relatively short reads $\sim 250 \mathrm{bp}$ ), as expected with the technical improvements of the sequencing services nowadyas (long reads $\sim 500 \mathrm{bp}$ ). These limitations suggested that only a few 
microsatellite loci within the DNA sequence file would be suitable for primer design because most of the repeats would have short (or no) flanking sequence regions.

A search of the sequences using MSATCOMMANDER found 3,082 sequences that contained perfect microsatellite-like repeat motifs; 2442 dinucleotide, 271 trinucleotide, 298 tetranucleotide, 71 pentanucleotide and one hexanucleotide.

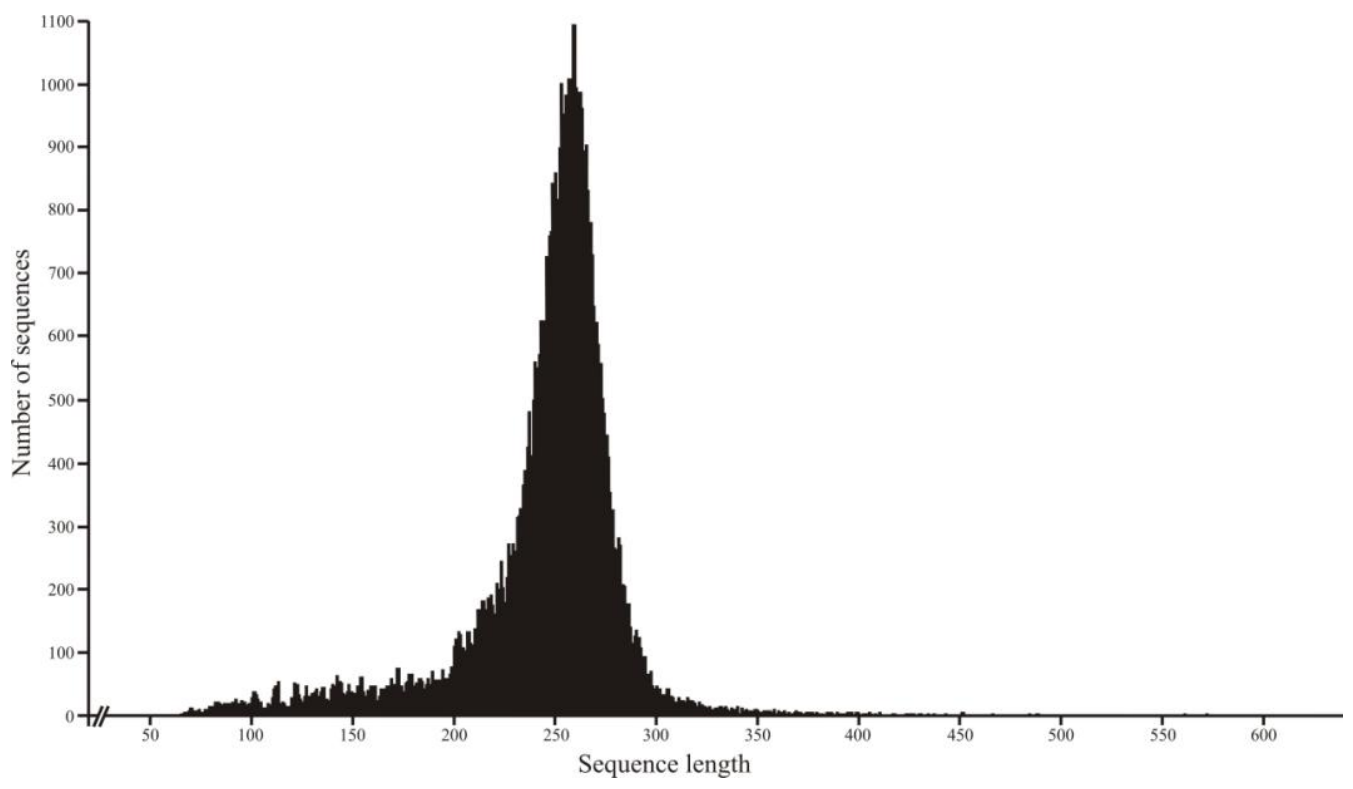

Figure 3.1: Distribution of sequence length of 46,602 DNA readings obtained from NGS (Roche 454).

The search for acceptable microsatellite-containing sequence using QDD found 15 loci that were recommended for further assessment. Most of these 15 loci were dinucleotide-type repeats: five loci contained simple repeats ( $g$ gal1 , 2, 3, 5, 16), two loci contained compound simple repeat (Ggal10, 11), and seven loci contained imperfect repeats. One locus (Ggal15) contained a trinucleotide repeat (Table 3.1). The test of the 15 loci using over 200 DNA samples showed that only three loci were polymorphic $($ Ggal8, 14, 15) and the numbers of alleles range from 2-9 (see Table 3.1). The observed heterozygosity ranged between 0.135 for Ggal8 and 0.510 for Ggal15 and the expected heterozygosity between 0.169 for Ggal8 and 0.598 for Ggal15. After correction for multiple tests, all three polymorphic loci deviated significantly from HWE because of heterozygote deficiency. A test of linkage disequilibrium showed that all pairs of loci were unlinked. 
Table 3.1: Characteristics of 15 microsatellite loci for G. galeus. $N=$ sample size screened for each, $\mathrm{A}=$ number of alleles, $H_{\mathrm{o}}=$ observed heterozygosity, $H_{\mathrm{e}}=$ expected heterozygosity, and $F_{\mathrm{IS}}=$ inbreeding coefficient (negative values = heterozygote excess and positive values $=$ heterozygote deficiency). NA means not-applicable.

\begin{tabular}{|c|c|c|c|c|c|c|c|c|c|}
\hline Locus & Forward primer 5'-3' & Reverse primer 5'-3' & Motif & $N$ & Size (bp) & & $H_{\mathrm{o}}$ & $H_{\mathrm{e}}$ & $F_{\text {IS }}$ \\
\hline Ggal1 & CGAATGGCCTAATTCTGCTC & CCCGGGAACGTTTAGTTTTA & $(\mathrm{TC})_{13}$ & 205 & NA & 0 & NA & NA & NA \\
\hline Ggal2 & ACCCAGCCAGAGTCAGCACC & АССТCTCTATCCCCTCGAAGATGC & $(\mathrm{AG})_{12}$ & 212 & 117 & 1 & 0 & 0 & 0 \\
\hline Ggal3 & TCAGTGAGAAAGCCAAAGAGC & CGATGCTTCACACTCTGTCC & $(\mathrm{GA})_{9}$ & 205 & 194 & 1 & 0 & 0 & 0 \\
\hline Ggal5 & TTCTTTCCTTCTCGGGTGTG & GGTAATGGCAAAAGGAGTGC & $(\mathrm{AG})_{8}$ & 212 & NA & 0 & NA & NA & NA \\
\hline Ggal6 & AGAGGGGAACTGAGACACCA & TACAATCGACAGGGCAACAA & $(\mathrm{AG})_{4}^{8} \mathrm{AA}(\mathrm{AG})_{8}$ & 217 & NA & 0 & NA & NA & NA \\
\hline Ggal7 & CTGGCTGGTGTTCCAAGG & GCATAGTGGTGGTGTGCTGT & $(\mathrm{TC})_{2} \mathrm{AT}(\mathrm{CCT})_{2} \mathrm{CTT}(\mathrm{CCT})_{5}(\mathrm{GCT})_{2}$ & 205 & 183 & 1 & 0 & 0 & 0 \\
\hline Ggal8 & CGTGCTTGTGGCAAATTTTA & GCAGGAACAGCGAGTGATTA & $(\mathrm{CT})_{2} \mathrm{CA}(\mathrm{GT})_{2}(\mathrm{CT})_{6} \mathrm{CC}(\mathrm{GT})_{2}$ & 217 & $121-123$ & 3 & 0.135 & 0.169 & 0.203 \\
\hline Ggal9 & AGAGAGGGAACTGAGACACCA & TACAATCGACAGGGCAACAA & $(\mathrm{GA})_{5} \mathrm{AA}(\mathrm{GA})_{7}$ & 217 & NA & 0 & NA & NA & NA \\
\hline Ggal10 & ATTGGCAAAAGAAGCAATGG & GGCCTGTTCCTGTGCTGTAT & $(\mathrm{TG})_{6}(\mathrm{AG})_{3}$ & 212 & 154 & 1 & 0 & 0 & 0 \\
\hline Ggal11 & AATTTGGTGGCAATTTGACG & CTGGGATCGAGGTCTGAAAG & $(\mathrm{AG})_{7}(\mathrm{GA})_{2}$ & 217 & NA & 0 & NA & NA & NA \\
\hline Ggal12 & ATGGGGTAAGGAATGGAAGG & AGCACCAAGTAAGGGCAGAA & $(\mathrm{GA})_{2} \mathrm{TGA}(\mathrm{AG})_{2}(\mathrm{GGAG})_{2}$ & 205 & NA & 0 & NA & NA & NA \\
\hline Ggal13 & CCAAGGTTTGATTTGCCACT & CAGTTCCCGAGTCTGAGTCC & $(\mathrm{CA})_{2} \mathrm{AA}(\mathrm{CA})_{6}$ & 212 & 135 & 1 & 0 & 0 & 0 \\
\hline Ggal14 & AGTCAAAGGATGCTCGCAGT & GGGCGATTATTTGGATGAGA & $(\mathrm{AA})_{2} \mathrm{AC}(\mathrm{AG})_{7}$ & 217 & $117-125$ & 4 & 0.378 & 0.52 & 0.281 \\
\hline Ggal15 & GTGACCTCCATCACCGACTT & GCCTTTGTGAAGAGCCTCAA & $(\mathrm{CAA})_{8}$ & 205 & $132-159$ & 9 & 0.510 & 0.598 & 0.147 \\
\hline Ggal16 & TCACTCGAGTCCGTCCAAAT & CTGGGTGGACACTCAGGTTAG & $(\mathrm{AG})_{7}$ & 217 & NA & 0 & NA & NA & NA \\
\hline
\end{tabular}




\subsection{Discussion}

The present study reported only three microsatellite loci, and they had several limitations to identifying and developing microsatellites. These include: (a) the average read length was $\sim 250$, which is an important factor given that two-thirds of the microsatellite detected are too close to either fragment end to enable development of flanking PCR primers (Abdelkrim et al. 2009, Castoe et al. 2010, Csencsics et al. 2010, Lepais \& Bacles 2010, Parchman et al. 2010, Guichoux et al. 2011, Gardner et al. 2011); (b) the number of repeats is very low for the majority of microsatellite loci tested, with the exception of Ggal1 and Ggal2 (13 and 12 repeats respectively). This situation is critical to define which sequences actually represent microsatellites. It is known that more repeats have higher mutation rates, which ensures detection of polymorphisms (Weber 1990, Heist \& Gold 1999, Ellegren 2000, Petit et al. 2005, Kelkar et al. 2008, Guichoux et al. 2011, Gardner et al. 2011). The number of polymorphic loci, number of repeats, number of alleles and genetic diversity (heterozygosity) found in the present study were lower than the results reported in the majority of previous studies elasmobranch species, with some exceptions e.g. Carcharhinus plumbeus (Heist \& Gold 1999). Nevertheless, the development of three new microsatellite loci for G. galeus using NGS provides a valuable tool for describing genetic diversity, genetic population structuring, effective population sizes and parentage studies when combined with other previously published microsatellite loci from this species.

Thirty two published studies (including the present study) have reported novel microsatellite loci involving 29 elasmobranch species (Table 3.1 in Supplementary Material). Most of these studies have been of shark i.e. 25 species (Selachii) and only a few have been from the rays and skates i.e. 4 species (Batoidea). The hybridization selection and linker and ligation methodologies have been the most commonly employed microsatellite isolation techniques reported. Only six studies obtained genomic libraries for microsatellite development using the NGS approach, these were for the species Mustelus antarticus (Boomer \& Stow 2010), G. galeus (Chabot \& Nigenda 2011), M. henlei (Chabot 2012), Manta alfredi (Kashiwagi et al. 2012), Isurus oxyrinchus (Taguchi et al. 2013) and the present study. 
Microsatellite DNA accounts for three percent of the human genome. Dinucleotide repeats are the most abundant, followed by mono- and tetranucleotides repeat and tetranucleotide repeats. When considering all the possible dinucleotide repeat, $(\mathrm{CA})_{\mathrm{n}}$ is the most common, followed by $(\mathrm{AT})_{\mathrm{n}}$, $(\mathrm{GA})_{\mathrm{n}},(\mathrm{GC})_{\mathrm{n}}$ (Ellegren 2004). There are only four possible types of dinucleotide CA (or GT), GA (or CT), AT, and GC. Note that CA repeat is the AG repeat on the opposite strand, which means the only difference between these motifs is the direction that they were originally sequenced. The published literature on population genetic studies of sharks that used microsatellites loci comprised 14 studies (including the present study), which included 15 shark species (Table 3.2 in Supplementary Material). A total of 111 microsatellite loci have been reported, which consist of di-nucleotide repeat units $(93.7 \%)$, tri- $(0.9 \%)$, tetra- $(4.5 \%)$, and hexa- $(0.9 \%)$ (Figure 3.1 in Supplementary Material). Within the di-nucleotide, CA was the highest reported repeats class (72.1\%), GA (18.9\%), and the others were GC (1.8\%), and TA $(0.9 \%)$.

The majority of microsatellite loci are composed of di-nucleotide repeats in several classes i.e. Insecta, Teleostei, Mammalia and Plantae (Wang et al. 1994, Schug et al. 1998, Li et al. 2002, Perumal et al. 2008). This review of motif repeats in shark studies are consistent with other taxa, which indicated a high frequency of $(\mathrm{CA})_{\mathrm{n}}$, followed by $(\mathrm{AT})_{\mathrm{n}},(\mathrm{GA})_{\mathrm{n}},(\mathrm{GC})_{\mathrm{n}}($ Ellegren 2004). However, the most common motif types can also vary widely amongs taxa (Dieringer \& Schlotterer 2003, Gardner et al. 2011). Those studies are base upon genome sequencing projects rather than small amount of microsatellites loci reported for population structure studies in shark species. Nevertheless, the first genomic sequencing projects are already on progress for Raja erinacea, Squalus acanthias, and Rhincodon typus (Dudgeon 2012). These studies will provide more insight about, for example, the distribution and frequency of microsatellites repeats in elasmobranch genomes. Despite of this drawback, nevertheless this information showed a clear trend comparable with other species. 


\subsection{Appendices}

\section{Supplementary Material}

Supplementary Table 3.1: List of microsatellite loci reported in primer notes to date for elasmobranch species. Three methodology approaches were utilised to develop SSRs: hybridization selection (HS), linker and ligation procedures (LL), expressed sequence tags (ESTs), and Roche 454. The number of loci, number of repeat, observed heterozygosity $\left(H_{\mathrm{o}}\right)$, and expected heterozygosity $\left(H_{\mathrm{e}}\right)$ are reported. Number of repeats and alleles, and observed and expected heterozygosity are represented by ranges across loci.

\begin{tabular}{|c|c|c|c|c|c|c|c|}
\hline Species & Methodology & $\mathrm{N}^{\circ}$ loci & $\mathrm{N}^{\circ}$ of repeats & A & $H_{o}$ & $H_{e}$ & Reference \\
\hline \multicolumn{8}{|l|}{ Selachii } \\
\hline Carcharhinus plumbeus & HS and LL & 3 & $6-10$ & $2-3$ & NR & $0.07-0.54$ & Heist \& Gold 1999 \\
\hline C. plumbeus & HS and LL & 5 & $13-42$ & $4-39$ & $0.63-1$ & $0.57-0.96$ & Portnoy et. al. 2006 \\
\hline C. plumbeus & HS and LL & 5 & 16 & 12 & NR & 0.84 & Portnoy et. al. 2007 \\
\hline C. limbatus & HS and LL & 16 & $4-34$ & $1-22$ & $0-0.96$ & $0-0.94$ & Keeney \& Heist 2003 \\
\hline C. tilstoni & HS & 5 & $4-28$ & $4-24$ & $0.63-1$ & $0.57-0.96$ & Ovenden et. al. 2006 \\
\hline C. sorrah & HS & 5 & $2-19$ & $5-24$ & $0.12-0.82$ & $0.16-0.95$ & Ovenden et. al. 2006 \\
\hline Negaprion brevirostris & HS and LL & 3 & $20-25$ & $13-22$ & $0.72-0.90$ & $0.73-0.90$ & Feldheim et. al. 2001a \\
\hline N. brevirostris & HS and LL & 15 & $2-33$ & $1-43$ & $0-0.87$ & $0-0.90$ & Feldheim et. al. 2001b \\
\hline N. brevirostris & HS & 5 & $11-18$ & $9-23$ & $0.69-0.87$ & $0.66-0.88$ & DiBattista et. al. 2008 \\
\hline Sphyrna tiburo & HS & 4 & NR & $6-35$ & $0.51-0.87$ & $0.55-0.69$ & Chapman et. al. 2004 \\
\hline S. lewini & HS and LL & 15 & NR & $6-29$ & $0.40-0.91$ & $0.54-0.90$ & Nance et. al. 2009 \\
\hline Mustelus antarticus & Roche 454 & 12 & $3-19$ & $4-11$ & $0.32-0.95$ & $0.30-0.84$ & Boomer \& Stow 2010 \\
\hline M. henlei & Roche 454 & 10 & NR & $2-10$ & $0.24-0.89$ & $0.23-0.86$ & Chabot 2012 \\
\hline Galeorhinus galeus & Roche 454 & 13 & NR & $3-12$ & $0-0.86$ & $0-0.87$ & Chabot \& Nigenda 2011 \\
\hline G. galeus & Roche 454 & 15 & $1-13$ & $1-9$ & $0-0.51$ & $0-0.60$ & This study \\
\hline Prionace glauca & HS & 10 & $2-27$ & $3-35$ & $0.24-0.91$ & $0.27-0.95$ & Fitzpatrick et. al. 2011 \\
\hline P. glauca & HS and LL & 8 & $2-16$ & $2-14$ & $0.08-0.78$ & $0.08-0.94$ & Mendoça et. al. 2012 \\
\hline Isurus oxyrhinchus & HS and LL & 5 & $4-53$ & $14->57$ & $0.77->0.90$ & $0.82-0.96$ & Schrey \& Heist 2002 \\
\hline I. oxyrhinchus & Roche 454 & 15 & $6-16$ & $2-23$ & $0.08-0.88$ & $0.08-0.91$ & Taguchi et. al. 2013 \\
\hline Ginglymostoma cirratum & HS and LL & 9 & $3-26$ & $3-15$ & $0.17-0.90$ & $0.16-0.92$ & Heist et. al. 2003 \\
\hline Carcharodon carcharias & HS and LL & 5 & $3-23$ & $3-10$ & $0.45-0.95$ & $0.51-0.83$ & Pardini et. al. 2000 \\
\hline Squalus acanthias & HS and LL & 8 & $2-11$ & $2-9$ & $0.37-0.84$ & $0.51-0.82$ & McCauley et. al. 2004 \\
\hline Stegostoma fasciatum & HS & 14 & $6-33$ & $3-22$ & $0.40-0.97$ & $0.53-0.97$ & Dudgeon et. al. 2006 \\
\hline Carcharias taurus & HS and LL & 8 & $4-32$ & $21-30$ & $0.29-1$ & $0.28-092$ & Feldheim et. al. 2007 \\
\hline Chiloscyllium plagiosum & HS and LL & 12 & $3-28$ & $4-12$ & $0.23-0.91$ & $0.41-0.89$ & Ding et. al. 2009 \\
\hline Rhincodon typus & HS & 9 & $4-22$ & $2-17$ & $0.20-0.83$ & $0.21-0.86$ & Ramírez-Macías et. al. 2009 \\
\hline Hexanchus griseus & HS & 14 & NR & $10-69$ & $0.24-1$ & $0.76-0.96$ & Larson et. al. 2009 \\
\hline Scyliorhimus canicula & ESTs & 17 & $5-15$ & $2-12$ & $0.13-0.88$ & $0.12-0.83$ & Griffiths et. al. 2011 \\
\hline Centroscymnus coelolepis & HS and LL & 10 & $6-35$ & $3-29$ & $0.60-0.98$ & $0.59-0.96$ & Veríssimo et. al. 2011 \\
\hline Centroselachus crepidater & HS and LL & 13 & $3-30$ & $3-7$ & $0.18-0.95$ & $0.25-0.82$ & Helyar et. al. 2011 \\
\hline \multicolumn{8}{|l|}{ Batoidea } \\
\hline Raja clavata & HS & 5 & $2-21$ & $8-48$ & $0.36-0.94$ & $0.32-0.98$ & Chevolot et. al. 2005 \\
\hline Aetobatus flagellum & HS and LL & 8 & $6-22$ & $2-6$ & $0.21-0.79$ & $0.20-0.71$ & Yagushita \& Yamaguchi 2009 \\
\hline A. narinari & HS and LL & 10 & $6-40$ & $2-19$ & $0.35-0.97$ & $0.33-0.95$ & Sellas et. al. 2011 \\
\hline Manta alfredi & Roche 454 & 10 & $6-18$ & $2-10$ & $0.22-0.87$ & $0.20-0.80$ & Kashiwagi et. al. 2012 \\
\hline
\end{tabular}


Supplementary Table 3.2: Genetic diversity from microsatellite loci reported to date for population genetic studies among 15 shark species by location. Sampling size $(N)$, number of alleles (A), observed heterozygosity $\left(H_{\mathrm{o}}\right)$, and expected heterozygosity $\left(H_{\mathrm{e}}\right)$ are reported. For Negaprion brevirostris and $N$. acutensis the allele richness i.e. asterisk "*” is represented rather than number of alleles due they were not reported by each loci in Schultz et al. 2008. For Squalus acanthias the number of alleles is represented across eight loci, for this reason is only one value.

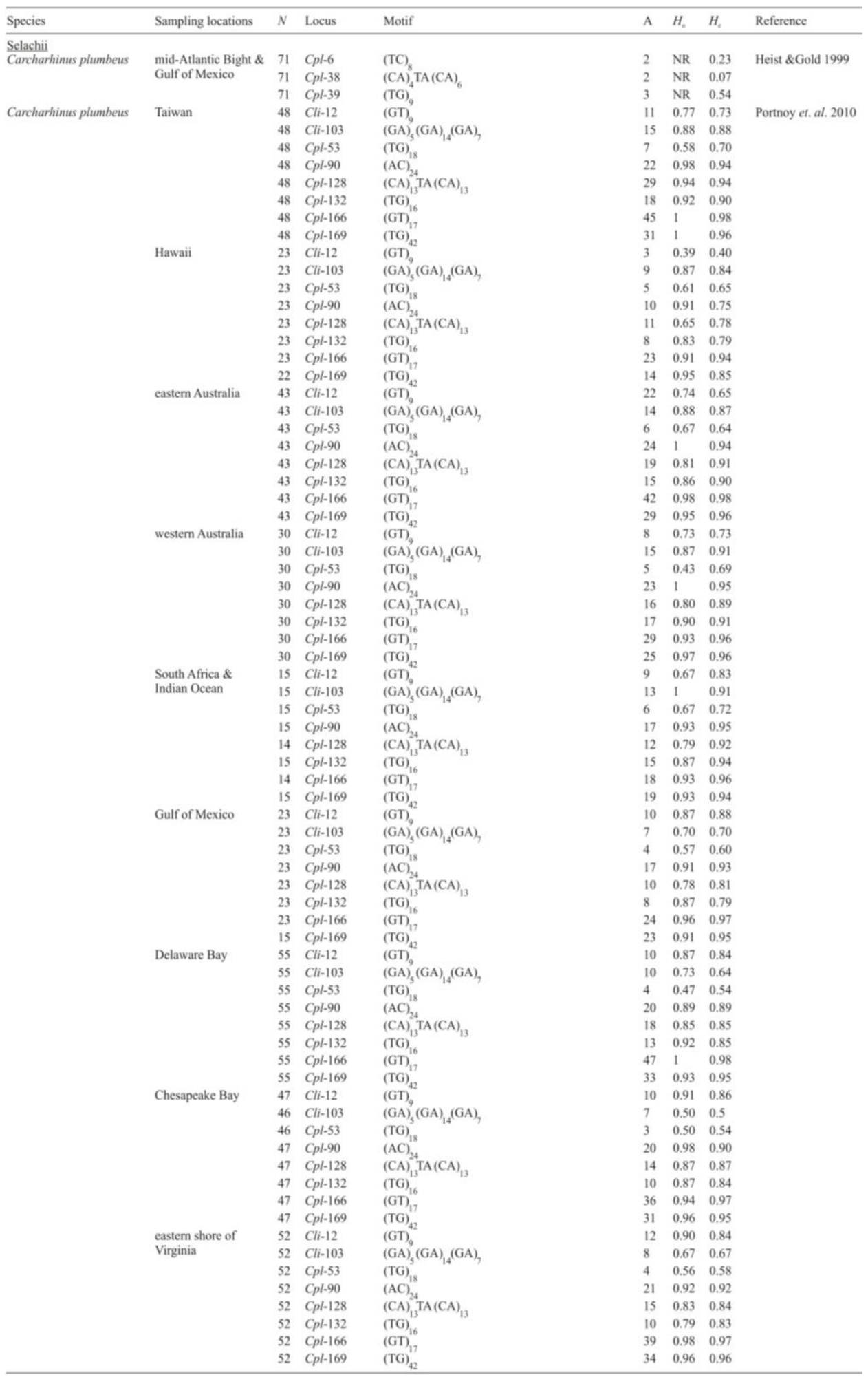




\begin{tabular}{|c|c|c|c|c|c|}
\hline Species & Sampling locations & $N$ & Locus & Motif & A \\
\hline Carcharhinus limbatus & Bull Bay South & 34 & Cli-2 & $(\mathrm{GA})_{10}$ & 1 \\
\hline & Carolina & 34 & Cli-7 & $(\mathrm{GT})_{20}$ & 24 \\
\hline & & 34 & $C l i-12$ & (GT) & 2 \\
\hline & & 34 & $C l i-13$ & $(\mathrm{GT})_{19}$ & 18 \\
\hline & & 34 & Cli-55 & (GT) 10 & 7 \\
\hline & & 34 & $C l i-100$ & $(\mathrm{TG})_{4}(\mathrm{GT})_{10}$ & 4 \\
\hline & & 34 & $C l i-107$ & $(\mathrm{GT})_{14}$ & 6 \\
\hline & & 34 & Cli-108 & (GT) 12 & 7 \\
\hline & Georgia & 13 & Cli-2 & $(\mathrm{GA})_{10}$ & 2 \\
\hline & & 13 & Cli-7 & $(\mathrm{GT})_{20}$ & 18 \\
\hline & & 13 & $C l i-12$ & (GT) ${ }_{9}^{20}$ & 3 \\
\hline & & 13 & $C l i-13$ & (GT) & 10 \\
\hline & & 13 & Cli-55 & (GT) 10 & 5 \\
\hline & & 13 & Cli-100 & $(\mathrm{TG})_{4}(\mathrm{GT})_{10}$ & 1 \\
\hline & & 13 & Cli-107 & $(\mathrm{GT})_{14}^{4}$ & 4 \\
\hline & & 13 & $C l i-108$ & (GT) $)_{12}$ & 8 \\
\hline & Pine Island Sound & 72 & Cli-2 & $(\mathrm{GA})_{10}^{12}$ & 4 \\
\hline & Florida & 72 & Cli-7 & $(\mathrm{GT})_{20}$ & 34 \\
\hline & & 72 & $C l i-12$ & $(\mathrm{GT})_{9}^{20}$ & 3 \\
\hline & & 72 & $C l i-13$ & (GT) & 21 \\
\hline & & 72 & Cli-55 & (GT) $)_{10}^{19}$ & 5 \\
\hline & & 72 & $C l i-100$ & $(\mathrm{TG})_{4}(\mathrm{GT})_{10}$ & 4 \\
\hline & & 72 & Cli-107 & $(\mathrm{GT})_{14}$ & 4 \\
\hline & & 72 & $C l i-108$ & $(\mathrm{GT})_{12}$ & 8 \\
\hline & Terra Ceia Bay & 71 & Cli-2 & $(\mathrm{GA})_{10}^{12}$ & 4 \\
\hline & Florida & 71 & Cli-7 & $(\mathrm{GT})_{20}$ & 31 \\
\hline & & 71 & $C l i-12$ & (GT) ${ }_{9}$ & 3 \\
\hline & & 71 & $C l i-13$ & (GT) & 21 \\
\hline & & 71 & Cli-55 & (GT) ${ }_{10}^{19}$ & 5 \\
\hline & & 71 & $C l i-100$ & $(\mathrm{TG})_{4}(\mathrm{GT})_{10}$ & 4 \\
\hline & & 71 & Cli-107 & $(\mathrm{GT})_{14}$ & 5 \\
\hline & & 71 & Cli-108 & $(\mathrm{GT})_{12}$ & 8 \\
\hline & Yankeetown Florida & 66 & $C l i-2$ & $(\mathrm{GA})_{10}$ & 3 \\
\hline & & 66 & Cli-7 & $(\mathrm{GT})_{20}$ & 33 \\
\hline & & 66 & $C l i-12$ & $\left(\mathrm{GT}_{9}\right.$ & 3 \\
\hline & & 66 & $C l i-13$ & (GT) & 21 \\
\hline & & 66 & Cli-55 & (GT) ${ }_{10}$ & 5 \\
\hline & & 66 & $C l i-100$ & $(\mathrm{TG})_{4}(\mathrm{GT})_{10}$ & 4 \\
\hline & & 66 & Cli-107 & $(\mathrm{GT})_{14}$ & 5 \\
\hline & & 66 & Cli-108 & $(\mathrm{GT})_{12}$ & 7 \\
\hline & Padre Island Texas & 50 & Cli-2 & $(\mathrm{GA})_{10}$ & 3 \\
\hline & & 50 & Cli-7 & $(\mathrm{GT})_{20}$ & 29 \\
\hline & & 50 & $C l i-12$ & (GT) & 3 \\
\hline & & 50 & $C l i-13$ & (GT) & 17 \\
\hline & & 50 & Cli-55 & (GT) ${ }_{10}$ & 7 \\
\hline & & 50 & $C l i-100$ & $(\mathrm{TG})_{4}(\mathrm{GT})_{10}$ & 4 \\
\hline & & 50 & Cli-107 & $(\mathrm{GT})_{14}$ & 5 \\
\hline & & 50 & $C l i-108$ & $(\mathrm{GT})_{12}$ & 7 \\
\hline & Laguna Yalahau & 84 & Cli-2 & $(\mathrm{GA})_{10}$ & 4 \\
\hline & northern Yucatan & 84 & Cli-7 & $(\mathrm{GT})_{20}$ & 31 \\
\hline & & 84 & $C l i-12$ & $(\mathrm{GT})_{9}^{20}$ & 3 \\
\hline & & 84 & $C l i-13$ & (GT) & 25 \\
\hline & & 84 & Cli-55 & (GT) & 7 \\
\hline & & 84 & $C l i-100$ & $(\mathrm{TG})_{4}(\mathrm{GT})_{10}$ & 4 \\
\hline & & 84 & $C l i-107$ & $(\mathrm{GT})_{14}^{4}$ & 6 \\
\hline & & 84 & Cli-108 & $(\mathrm{GT})_{12}$ & 8 \\
\hline & Belize City & 10 & $\mathrm{Cli}-2$ & $(\mathrm{GA})_{10}$ & 3 \\
\hline & & 10 & Cli-7 & (GT) $)_{20}^{10}$ & 10 \\
\hline & & 10 & $C l i-12$ & (GT) & 1 \\
\hline & & 10 & $C l i-13$ & (GT) & 9 \\
\hline & & 10 & Cli-55 & (GT) & 2 \\
\hline & & 10 & $C l i-100$ & $(\mathrm{TG})_{4}(\mathrm{GT})_{10}$ & 3 \\
\hline & & 10 & Cli-107 & $(\mathrm{GT})_{14}^{+}$ & 4 \\
\hline & & 10 & $C l i-108$ & $(\mathrm{GT})_{12}$ & 3 \\
\hline & Dangriga & 19 & Cli-2 & $(\mathrm{GA})_{10}$ & 3 \\
\hline & & 19 & Cli-7 & $(\mathrm{GT})_{20}$ & 20 \\
\hline & & 19 & Cli-12 & $(\mathrm{GT})_{9}^{20}$ & 3 \\
\hline & & 19 & Cli-13 & (GT) & 17 \\
\hline & & 19 & Cli-55 & (GT) & 4 \\
\hline & & 19 & $C l i-100$ & $(\mathrm{TG})_{4}(\mathrm{GT})_{10}$ & 4 \\
\hline & & 19 & Cli-107 & $\left(\mathrm{GT}^{4}\right)_{14}^{4}$ & 5 \\
\hline & & 19 & $C l i-108$ & (GT) & 5 \\
\hline Carcharhimus sorrah & Indonesia & 31 & $L S-15$ & $(\mathrm{AC})_{20}$ & 2 \\
\hline & & 25 & $C_{t}-5$ & (GT) $)_{10}^{20}$ & 10 \\
\hline & & 47 & $C s-12$ & (GT) $)_{19}$ & 5 \\
\hline & & 42 & $C l i-100$ & $(\mathrm{TG})_{4}(\mathrm{GT})_{10}$ & 4 \\
\hline & & 28 & $C s-8$ & $(\mathrm{CA})_{28}^{+}$ & 24 \\
\hline & west Australia & 53 & $L S-15$ & $(\mathrm{AC})_{20}$ & 4 \\
\hline & & 51 & $C t-5$ & $(\mathrm{GT})_{19}^{20}$ & 17 \\
\hline & & 51 & $C s-12$ & (GT) 19 & 7 \\
\hline & & 46 & $C l i-100$ & $(\mathrm{TG})_{4}(\mathrm{GT})_{10}$ & 8 \\
\hline & & 40 & $C s-8$ & $(\mathrm{CA})_{28}$ & 27 \\
\hline & east Australia & 37 & $L S-15$ & $(\mathrm{AC})_{20}^{20}$ & 2 \\
\hline & & 37 & $C t-5$ & (GT) & 10 \\
\hline & & 36 & $C s-12$ & (GT) $)_{19}$ & 7 \\
\hline & & 34 & $C l i-100$ & $(\mathrm{TG})_{4}(\mathrm{GT})_{10}$ & 4 \\
\hline & & 29 & Cs-8 & $(\mathrm{CA})_{28}^{1}$ & 24 \\
\hline & Gulf of Carpentaria & 57 & $L S-15$ & $(\mathrm{AC})_{20}$ & 6 \\
\hline & & 46 & $C t-5$ & (GT) & 13 \\
\hline & & 67 & $C s-12$ & (GT) & 6 \\
\hline & & 61 & $C l i-100$ & $(\mathrm{TG})_{4}(\mathrm{GT})_{10}$ & 6 \\
\hline & & 48 & Cs-8 & $(\mathrm{CA})_{28}$ & 30 \\
\hline
\end{tabular}




\begin{tabular}{|c|c|c|c|c|c|c|c|}
\hline Species & Sampling locations & $N$ & Locus & Motif & A & $H_{\circ}$ & $H_{e}$ \\
\hline Carcharhimus obscurus & Indonesia & 22 & Cli-108 & $(\mathrm{GT})_{12}$ & 4 & 0.73 & 0.69 \\
\hline & & 18 & $C_{s-2}$ & $(\mathrm{GT}),(\mathrm{GC})(\mathrm{GT})(\mathrm{GC})(\mathrm{GT})_{1}$ & 14 & 0.56 & 0.88 \\
\hline & & 17 & $C s-6$ & $(\mathrm{CA})_{9}^{2} \mathrm{AA}(\mathrm{CA})$ & 16 & 0.88 & 0.86 \\
\hline & & 25 & $L S-24$ & $(\mathrm{AC})_{12}^{9}$ & 3 & 0.44 & 0.52 \\
\hline & west Australia & 66 & Cli-108 & $(\mathrm{GT})_{12}^{12}$ & 4 & 0.58 & 0.59 \\
\hline & & 48 & $C s-2$ & $(\mathrm{GT})_{2}(\mathrm{GC})(\mathrm{GT})(\mathrm{GC})(\mathrm{GT})_{1}$ & 15 & 0.42 & 0.90 \\
\hline & & 27 & $C s-6$ & $(\mathrm{CA})^{2} \mathrm{AA}(\mathrm{CA})$ & 16 & 0.89 & 0.88 \\
\hline & & 59 & $L S-24$ & $(\mathrm{AC})_{12}^{\prime}$ & 4 & 0.54 & 0.56 \\
\hline & east Australia & 5 & Cli-108 & $(\mathrm{GT})_{12}$ & 4 & 0.80 & 0.70 \\
\hline & & 4 & $C_{s-2}$ & $(\mathrm{GT}),(\mathrm{GC})(\mathrm{GT})(\mathrm{GC})(\mathrm{GT})$ & 4 & 0.50 & 0.72 \\
\hline & & 5 & $C s-6$ & $(\mathrm{CA})_{0}^{2} \mathrm{AA}(\mathrm{CA})$ & 8 & 1 & 0.86 \\
\hline & & 7 & $L S-24$ & $(\mathrm{AC})^{3}$ & 3 & 0.14 & 0.56 \\
\hline Carcharhimus leucas & north Carolina & 19 & Cli-7 & $(\mathrm{GT})_{20}$ & 19 & 1 & 0.95 \\
\hline & & 19 & Cli-107 & $(\mathrm{GT})^{20}$ & 13 & 0.90 & 0.86 \\
\hline & & 19 & Cli-106 & $(\mathrm{GA})^{14}$ & 5 & 0.68 & 0.65 \\
\hline & & 19 & Cli-108 & $(\mathrm{TG})_{4}(\mathrm{GT})_{10}$ & 16 & 0.95 & 0.92 \\
\hline & & 19 & Cli-112 & $(\mathrm{GT})_{4}(\mathrm{GT})_{14}$ & 23 & 0.95 & 0.97 \\
\hline & Gulf coast of Florida & 35 & Cli-7 & $(\mathrm{GT})_{20}$ & 31 & 0.94 & 0.96 \\
\hline & & 34 & Cli-107 & $(\mathrm{GT})^{20}$ & 15 & 0.91 & 0.87 \\
\hline & & 35 & Cli-106 & $(\mathrm{GA})^{14}$ & 4 & 0.37 & 0.43 \\
\hline & & 33 & Cli-108 & $(\mathrm{GT})_{12}$ & 24 & 0.97 & 0.91 \\
\hline & & 34 & Cli-112 & $(\mathrm{GT})_{4}^{12}(\mathrm{GT})_{14}$ & 28 & 0.88 & 0.97 \\
\hline & northern Gulf & 36 & $\mathrm{Cli}-7$ & $(\mathrm{GT})_{0}^{4} \quad 14$ & 25 & 0.94 & 0.95 \\
\hline & & 36 & Cli-107 & $(\mathrm{GT})_{14}^{20}$ & 18 & 0.89 & 0.84 \\
\hline & & 33 & Cli-106 & $(\mathrm{GA})^{14}$ & 5 & 0.49 & 0.42 \\
\hline & & 27 & Cli-108 & $(\mathrm{GT})_{12}^{11}$ & 15 & 0.93 & 0.90 \\
\hline & & 28 & $C l i-112$ & $(\mathrm{GT})(\mathrm{GT})$ & 28 & 0.71 & 0.97 \\
\hline & Brazil & 54 & Cli-7 & $(\mathrm{GT})^{4}$ & 28 & 0.91 & 0.95 \\
\hline & & 55 & Cli-107 & (GT) & 23 & 0.84 & 0.87 \\
\hline & & 45 & Cli-106 & $(\mathrm{GA})^{14}$ & 4 & 0.64 & 0.60 \\
\hline & & 55 & Cli-108 & $(\mathrm{GT})_{12}^{11}$ & 29 & 0.98 & 0.95 \\
\hline & & 47 & Cli-112 & $(\mathrm{GT})_{4}(\mathrm{GT})_{14}$ & 30 & 0.79 & 0.96 \\
\hline Negaprion brevinstris & middle and southern & 545 & $L S-11$ & $(\mathrm{AC})_{33}^{4}$ & 43 & 0.68 & 0.69 \\
\hline & Atlantic Ocean & 688 & $L S-15$ & $(\mathrm{AC})_{20}^{33}$ & 30 & 0.79 & 0.80 \\
\hline & & 673 & $L S-22$ & $(\mathrm{AC})_{23}$ & 22 & 0.87 & 0.90 \\
\hline & & 726 & $L S-30$ & $(\mathrm{AC})_{2}^{23}$ & 19 & 0.73 & 0.75 \\
\hline N. brevirostris & Bimini, Bahamas & 30 & $L S-11$ & $(\mathrm{AC})_{33}^{23}$ & $6^{*}$ & 0.59 & $\mathrm{NR}$ \\
\hline & & 30 & $L S-15$ & $(\mathrm{AC})_{0}^{33}$ & $9^{*}$ & 0.80 & NR \\
\hline & & 30 & $L S-48$ & $(\mathrm{AC})_{38}^{20}$ & $12^{*}$ & 0.95 & NR \\
\hline & & 30 & $L S-52$ & $(\mathrm{AC})_{20}^{38}$ & $13^{*}$ & 0.95 & NR \\
\hline & & 30 & $L S-54$ & $(\mathrm{CT})^{20}(\mathrm{CA})$ & $3^{*}$ & 0.46 & NR \\
\hline & & 30 & $L S-22$ & $(\mathrm{AC})_{23}$ & 9* & 0.89 & NR \\
\hline & & 30 & $L S-30$ & $(\mathrm{AC})_{2}^{23}$ & $6^{*}$ & 0.67 & NR \\
\hline & & 30 & $L S-75$ & $(\mathrm{TC})_{11}^{25}(\mathrm{AC})_{11} \mathrm{AG}(\mathrm{AC})_{10}$ & $4^{*}$ & 0.68 & NR \\
\hline & & 30 & $L S-82$ & $(\mathrm{AC})_{25}$ & $8^{*}$ & 0.78 & NR \\
\hline & Brazil & 30 & $L S-11$ & $(\mathrm{AC})_{33}^{5}$ & $6^{*}$ & 0.59 & NR \\
\hline & & 30 & $L S-15$ & $(\mathrm{AC})_{20}^{33}$ & $6^{*}$ & 0.67 & NR \\
\hline & & 30 & $L S-48$ & $(\mathrm{AC})_{3 \mathrm{~B}}$ & $10^{*}$ & 0.92 & NR \\
\hline & & 30 & $L S-52$ & $(\mathrm{AC})_{20}^{88}$ & $11^{*}$ & 0.94 & NR \\
\hline & & 30 & $L S-54$ & $(\mathrm{CT})_{10}^{20}(\mathrm{CA})_{8}$ & $4^{*}$ & 0.57 & $\mathrm{NR}$ \\
\hline & & 30 & $L S-22$ & $(\mathrm{AC})_{23}$ & $8^{*}$ & 0.88 & NR \\
\hline & & 30 & $L S-30$ & $(\mathrm{AC})_{25}^{23}$ & $5^{*}$ & 0.75 & NR \\
\hline & & 30 & $L S-75$ & $(\mathrm{TC})(\mathrm{AC}) \mathrm{AG}(\mathrm{AC})_{10}$ & $4^{*}$ & 0.54 & NR \\
\hline & & 30 & $L S-82$ & $(\mathrm{AC})_{25}$ & $8^{*}$ & 0.76 & NR \\
\hline & Guinea-Bissau & 11 & $L S-11$ & $(\mathrm{AC})_{33}^{23}$ & $1^{*}$ & 0 & NR \\
\hline & & 11 & $L S-15$ & (AC) & $11^{*}$ & 0.93 & NR \\
\hline & & 11 & $L S-48$ & $(\mathrm{AC})_{88}^{20}$ & $11^{*}$ & 0.94 & NR \\
\hline & & 11 & $L S-52$ & $(\mathrm{AC})_{20}$ & $13^{*}$ & 0.97 & NR \\
\hline & & 11 & $L S-54$ & $(\mathrm{CT})_{10}^{20}(\mathrm{CA})_{8}$ & $1^{*}$ & 0 & NR \\
\hline & & 11 & $L S-22$ & $(\mathrm{AC})_{31}$ & $5^{*}$ & 0.61 & NR \\
\hline & & 11 & $L S-30$ & $(\mathrm{AC})_{25}^{23}$ & $7^{*}$ & 0.79 & NR \\
\hline & & 11 & $L S-75$ & $(\mathrm{TC})_{11}^{25}(\mathrm{AC})_{11} \mathrm{AG}(\mathrm{AC})_{10}$ & $2^{*}$ & 0.09 & NR \\
\hline & & 11 & $L S-82$ & (AC) & $4^{*}$ & 0.41 & NR \\
\hline & Mexico & 9 & $L S-11$ & $(\mathrm{AC})_{33}^{25}$ & $3^{*}$ & 0.68 & NR \\
\hline & & 9 & $L S-15$ & $\left(\mathrm{AC}_{20}\right.$ & $5^{*}$ & 0.71 & NR \\
\hline & & 9 & $L S-48$ & $(\mathrm{AC})_{3 \mathrm{~K}}^{20}$ & $11^{*}$ & 0.93 & NR \\
\hline & & 9 & $L S-52$ & $(\mathrm{AC})_{\gamma 0}^{38}$ & $13^{*}$ & 0.96 & NR \\
\hline & & 9 & $L S-54$ & $(\mathrm{CT})_{10}^{20}(\mathrm{CA})_{8}$ & $3^{*}$ & 0.54 & $\mathrm{NR}$ \\
\hline & & 9 & $L S-22$ & $(\mathrm{ACC})_{23}$ & $7^{*}$ & 0.82 & NR \\
\hline & & 9 & $L S-30$ & $(\mathrm{AC})_{2}^{23}$ & $8^{*}$ & 0.85 & NR \\
\hline & & 9 & $L S-75$ & (TC) ${ }^{25}(\mathrm{AC}), \mathrm{AG}(\mathrm{AC})$ & $3^{*}$ & 0.57 & NR \\
\hline & & 9 & $L S-82$ & $(\mathrm{AC})_{25}$ & $11^{*}$ & 0.86 & NR \\
\hline N. acutidens & western Australia & 25 & $L S-11$ & $(\mathrm{AC})_{33}^{25}$ & $3^{*}$ & 0.77 & NR \\
\hline & & 25 & $L S-15$ & $(\mathrm{AC})_{20}^{33}$ & $3^{*}$ & 0.78 & $\mathrm{NR}$ \\
\hline & & 25 & $L S-52$ & $(\mathrm{AC})_{20}^{20}$ & $3^{*}$ & 0.81 & NR \\
\hline & & 25 & $L S-54$ & $(\mathrm{CT})_{10}^{20}(\mathrm{CA})_{8}$ & $1^{*}$ & 0 & NR \\
\hline & & 25 & $L S-75$ & (TC) $(\mathrm{AC}) \mathrm{AG}(\mathrm{AC})$ & $3^{*}$ & 0.86 & NR \\
\hline & & 25 & $L S-82$ & $(\mathrm{AC})_{25}$ & $2^{*}$ & 0.41 & NR \\
\hline & eastern Australia & 10 & $L S-11$ & $(\mathrm{AC})_{33}$ & $3^{*}$ & 0.71 & NR \\
\hline & & 10 & $L S-15$ & $(\mathrm{AC})_{20}^{33}$ & $2^{*}$ & 0.58 & NR \\
\hline & & 10 & $L S-52$ & $(\mathrm{AC})_{0}^{20}$ & $4^{*}$ & 0.92 & NR \\
\hline & & 10 & $L S-54$ & (CT) ${ }_{10}(\mathrm{CA})_{8}$ & $2^{*}$ & 0.56 & NR \\
\hline & & 10 & $L S-75$ & (TC) $(\mathrm{AC}) \mathrm{AG}(\mathrm{AC})$ & $3^{*}$ & 0.82 & NR \\
\hline & & 10 & $L S-82$ & $(\mathrm{AC})_{25}$ & $2^{*}$ & 0.53 & NR \\
\hline & New Caledonia & 2 & $L S-11$ & $(\mathrm{AC})_{33}^{-3}$ & $1^{*}$ & 0 & NR \\
\hline & & 2 & $L S-15$ & $(\mathrm{AC})_{0}^{33}$ & $2^{*}$ & 0.67 & NR \\
\hline & & 2 & $L S-52$ & $(\mathrm{AC})_{0}^{20}$ & $2^{*}$ & 0.67 & NR \\
\hline & & 2 & $L S-54$ & $(\mathrm{CT})_{0}^{20}(\mathrm{CA})_{8}$ & $1^{*}$ & 0 & NR \\
\hline & & 2 & $L S-75$ & $(\mathrm{TC})_{11}^{10}(\mathrm{AC})_{11}^{8} \mathrm{AG}(\mathrm{AC})_{10}$ & $2^{*}$ & 0.50 & NR \\
\hline & & 2 & $L S-82$ & $(\mathrm{AC})_{25}$ & $2^{*}$ & 0.50 & NR \\
\hline
\end{tabular}




\begin{tabular}{|c|c|c|c|c|c|c|}
\hline Species & Sampling locations & $N$ & Locus & Motif & $\mathrm{A}$ & $H_{*}$ \\
\hline N. acutidens & French Polynesia & 16 & $L S-11$ & $(\mathrm{AC})_{33}$ & $2^{*}$ & 0.63 \\
\hline & & 16 & $L S-15$ & $(\mathrm{AC})_{20}^{33}$ & $2^{*}$ & 0.50 \\
\hline & & 16 & $L S-52$ & $(\mathrm{AC})_{20}^{20}$ & $1^{*}$ & 0 \\
\hline & & 16 & $L S-54$ & $(\mathrm{CT})_{10}^{20}(\mathrm{CA})_{8}$ & $2^{*}$ & 0.54 \\
\hline & & 16 & $L S-75$ & (TC) $(\mathrm{AC})^{\mathrm{AG}(\mathrm{AC})}$ & $3^{*}$ & 0.70 \\
\hline & & 16 & $L S-82$ & $(\mathrm{AC})_{25}$ & $2^{*}$ & 0.29 \\
\hline & Taiwan & 15 & $L S-11$ & $(\mathrm{AC})_{33}^{23}$ & $3^{*}$ & 0.76 \\
\hline & & 15 & $L S-15$ & $(\mathrm{AC})_{20}$ & $3^{*}$ & 0.84 \\
\hline & & 15 & $L S-52$ & $(\mathrm{AC})_{20}^{20}$ & $2^{*}$ & 0.38 \\
\hline & & 15 & $L S-54$ & (CT) $)_{10}(\mathrm{CA})_{8}$ & $2^{*}$ & 0.38 \\
\hline & & 15 & $L S-75$ & (TC) $(\mathrm{AC}) \mathrm{AG}(\mathrm{AC})$ & $3^{*}$ & 0.89 \\
\hline & & 15 & $L S-82$ & $(\mathrm{AC})_{25}$ & $2^{*}$ & 0.53 \\
\hline Sphyrna lewini & Indonesia & 74 & $C l-6$ & (CA) & 1 & 0 \\
\hline & & 74 & $C t-7$ & $(\mathrm{GT})(\mathrm{GC})(\mathrm{GT})(\mathrm{GC})(\mathrm{GT})$ & 6 & 0.66 \\
\hline & & 82 & Cli-100 & $(\mathrm{TG})_{4}(\mathrm{GT})_{10}$ & 14 & 0.82 \\
\hline & east Australia & 44 & $C t-6$ & $(\mathrm{CA})_{14}$ & 1 & 0 \\
\hline & & 44 & $C t-7$ & $(\mathrm{GT}){ }_{10}(\mathrm{GC})(\mathrm{GT})(\mathrm{GC})(\mathrm{GT})_{2}$ & 6 & 0.80 \\
\hline & & 44 & Cli-100 & $(\mathrm{TG})_{4}(\mathrm{GT})_{10}$ & 10 & 0.80 \\
\hline & west Australia & 42 & $C_{t-6}$ & $(\mathrm{CA})_{14}$ & 2 & 0 \\
\hline & & 31 & $C t-7$ & $(\mathrm{GT})_{10}(\mathrm{GC})(\mathrm{GT})(\mathrm{GC})(\mathrm{GT})_{2}$ & 7 & 0.61 \\
\hline & & 40 & $C l i-100$ & $(\mathrm{TG})_{4}(\mathrm{GT})_{10}$ & 11 & 0.75 \\
\hline Ginglymostoma cirratum & Brazilian islands & 41 & $G c-4$ & $(\mathrm{CA})_{3}^{7}(\mathrm{CAGA})_{5}(\mathrm{GT})_{9}$ & 3 & 0.34 \\
\hline & & 39 & $G c-7$ & (GT) ${ }_{7}^{\mathrm{TT}}(\mathrm{GT})_{5}$ & 6 & 0.69 \\
\hline & & 41 & $G c-10$ & $(\mathrm{GT})_{26}$ & 20 & 0.83 \\
\hline & & 40 & $G c-11$ & $(\mathrm{GT})_{12}^{60}$ & 3 & 0.30 \\
\hline & & 42 & $G c-18$ & $(\mathrm{GT})_{9}^{2}$ & 3 & 0.31 \\
\hline & & 38 & $G c-25$ & $(\mathrm{GAGACA})_{6}(\mathrm{GC})_{2}(\mathrm{GA})_{3}$ & 7 & 0.71 \\
\hline & & 42 & $G c-35$ & $(\mathrm{GT})_{10}$ & 4 & 0.64 \\
\hline & & 42 & $G c-37$ & $(\mathrm{GA})_{15}$ & 6 & 0.63 \\
\hline & Rio Grande do Norte & 15 & $G c-4$ & $(\mathrm{CA})_{3}(\mathrm{CAGA})_{5}(\mathrm{GT})_{9}$ & 3 & 0.47 \\
\hline & & 14 & $G c-7$ & $(\mathrm{GT})_{7}^{3} \mathrm{TT}(\mathrm{GT})_{5}^{3}$ & 5 & 0.50 \\
\hline & & 14 & $G c-10$ & $(\mathrm{GT})_{26}$ & 9 & 0.71 \\
\hline & & 15 & $G c-11$ & $(\mathrm{GT})_{12}^{20}$ & 3 & 0.20 \\
\hline & & 14 & $G c-18$ & $(\mathrm{GT})_{9}^{2}$ & 3 & 0.64 \\
\hline & & 14 & $G c-25$ & $(\mathrm{GAGACA})_{6}(\mathrm{GC})_{2}(\mathrm{GA})_{13}$ & 4 & 0.86 \\
\hline & & 14 & $G c-35$ & $(\mathrm{GT})_{10}-{ }^{6}-2=13$ & 3 & 0.50 \\
\hline & & 15 & $G c-37$ & $(\mathrm{GA})_{15}^{10}$ & 5 & 0.80 \\
\hline & Bimini, Bahamas & 23 & $G c-4$ & $(\mathrm{CA})_{3}(\mathrm{CAGA})_{5}(\mathrm{GT})_{9}$ & 3 & 0.35 \\
\hline & & 23 & $G c-7$ & $(\mathrm{GT})_{7} \mathrm{TT}(\mathrm{GT})_{5}$ & 4 & 0.78 \\
\hline & & 23 & $G c-10$ & $(\mathrm{GT})_{26}^{\prime}$ & 16 & 0.91 \\
\hline & & 23 & $G c-11$ & $\left(\mathrm{GT}_{12}^{20}\right.$ & 3 & 0.52 \\
\hline & & 23 & $G c-18$ & $(\mathrm{GT})_{9}^{12}$ & 3 & 0.48 \\
\hline & & 23 & $G c-25$ & $(\mathrm{GAGACA})_{6}(\mathrm{GC})_{2}(\mathrm{GA})_{13}$ & 7 & 0.91 \\
\hline & & 23 & $G c-35$ & $(\mathrm{GT})_{10}-2=13$ & 4 & 0.52 \\
\hline & & 23 & $G c-37$ & $(\mathrm{GA})_{15}$ & 6 & 0.57 \\
\hline & Big Pine Island & 37 & $G c-4$ & $(\mathrm{CA})_{3}(\mathrm{CAGA})_{5}(\mathrm{GT})_{9}$ & 3 & 0.46 \\
\hline & & 36 & $G c-7$ & $(\mathrm{GT})_{7}^{3} \mathrm{TT}(\mathrm{GT})_{5}$ & 6 & 0.78 \\
\hline & & 36 & $G c-10$ & $(\mathrm{GT})_{26}$ & 18 & 0.92 \\
\hline & & 37 & $G c-11$ & $(\mathrm{GT})_{12}^{6}$ & 3 & 0.54 \\
\hline & & 37 & $G c-18$ & $(\mathrm{GT})_{9}^{2}$ & 3 & 0.46 \\
\hline & & 36 & $G c-25$ & $(\mathrm{GAGACA})_{6}(\mathrm{GC})_{2}(\mathrm{GA})_{3}$ & 9 & 0.64 \\
\hline & & 37 & $G c-35$ & $(\mathrm{GT})_{10}$ & 4 & 0.57 \\
\hline & & 35 & $G c-37$ & $(\mathrm{GA})_{15}$ & 6 & 0.43 \\
\hline & Caribbean coast of & 34 & $G c-4$ & $(\mathrm{CA})_{3}(\mathrm{CAGA})_{5}(\mathrm{GT})_{9}$ & 3 & 0.41 \\
\hline & Belize & 35 & $G c-7$ & (GT) ${ }_{7}^{3}(\mathrm{GT})_{4}$ & 6 & 0.71 \\
\hline & & 35 & $G c-10$ & $(\mathrm{GT})_{26}^{\prime}$ & 17 & 0.91 \\
\hline & & 35 & $G c-11$ & $(\mathrm{GT})_{12}^{26}$ & 3 & 0.37 \\
\hline & & 35 & $G c-18$ & $(\mathrm{GT})_{9}^{2}$ & 3 & 0.26 \\
\hline & & 35 & $G c-25$ & $(\mathrm{GAGACA})_{6}(\mathrm{GC})_{2}(\mathrm{GA})_{13}$ & 6 & 0.60 \\
\hline & & 35 & $G c-35$ & $(\mathrm{GT})_{10}{ }^{-6}-2^{2}-13$ & 4 & 0.49 \\
\hline & & 35 & $G c-37$ & $(\mathrm{GA})_{15}$ & 6 & 0.40 \\
\hline Prionace glauca & Indonesia & 26 & $C s-2$ & $(\mathrm{GT}),(\mathrm{GC})(\mathrm{GT})(\mathrm{GC})(\mathrm{GT})_{1}$ & 15 & 0.81 \\
\hline & & 33 & $C_{t-4}$ & $(\mathrm{CA})_{2}^{2}$ & 5 & 0.55 \\
\hline & & 30 & $\mathrm{Cl}_{\mathrm{t}-6}$ & (CA) ${ }_{14}^{1}$ & 6 & 0.40 \\
\hline & & 31 & Cli-107 & $(\mathrm{GT})_{14}^{4}$ & 2 & 0.26 \\
\hline & & 28 & $C l i-100$ & $(\mathrm{TG})_{4}^{4}(\mathrm{GT})_{10}$ & 5 & 0.36 \\
\hline & mid north Pacific & 20 & Cs-2 & $(\mathrm{GT})_{2}^{\prime}(\mathrm{GC})(\mathrm{GT})(\mathrm{GC})(\mathrm{GT})_{11}$ & 17 & 0.95 \\
\hline & & 20 & $C_{t-4}$ & $(\mathrm{CA})_{7}^{2}$ & 5 & 0.75 \\
\hline & & 20 & $C t-6$ & (CA) 14 & 6 & 0.55 \\
\hline & & 20 & Cli-107 & $(\mathrm{GT})_{14}^{4}$ & 3 & 0.15 \\
\hline & & 3 & Cli-100 & $(\mathrm{TG})_{4}^{4}(\mathrm{GT})_{10}$ & 2 & 0 \\
\hline & west Australia & 21 & $C s-2$ & $(\mathrm{GT})_{2}(\mathrm{GC})(\mathrm{GT})(\mathrm{GC})(\mathrm{GT})_{4}$ & 12 & 0.62 \\
\hline & & 47 & $C t-4$ & $(\mathrm{CA})_{7}^{2}$ & 5 & 0.70 \\
\hline & & 43 & $C l-6$ & $(\mathrm{CA})_{14}^{\prime}$ & 6 & 0.35 \\
\hline & & 42 & Cli-107 & $(\mathrm{GT})_{14}^{-4}$ & 4 & 0.31 \\
\hline & & 37 & Cli-100 & $(\mathrm{TG})_{4}(\mathrm{GT})_{10}$ & 5 & 0.30 \\
\hline & east Australia & 16 & $C s-2$ & $(\mathrm{GT})_{2}^{4}(\mathrm{GC})(\mathrm{GT})(\mathrm{GC})(\mathrm{GT})_{11}$ & 16 & 0.88 \\
\hline & & 18 & $C t-4$ & $(\mathrm{CA})_{7}^{2}$ & 5 & 0.56 \\
\hline & & 17 & $C_{1-6}$ & (CA) 14 & 5 & 0.41 \\
\hline & & 14 & Cli-107 & $(\mathrm{GT})_{14}^{-14}$ & 3 & 0.29 \\
\hline & & 16 & Cli-100 & $(\mathrm{TG})_{4}^{4}(\mathrm{GT})_{10}$ & 5 & 0.38 \\
\hline Cancharodon carcharias & New Zealand/Australia & 52 & Ccar -1 & $(\mathrm{AC})_{22}$ & 6 & 0.62 \\
\hline & & 52 & Ccar-6 & $(\mathrm{AC})_{17}^{2}$ & 4 & 0.70 \\
\hline & & 52 & Ccar-9 & $(\mathrm{TG})_{33}$ & 15 & 0.35 \\
\hline & & 52 & Ccar-13 & $(\mathrm{TG})_{4} \mathrm{TT}(\mathrm{TG})_{9} \mathrm{TT}(\mathrm{TG})_{3} \mathrm{TTTT}(\mathrm{TG})_{23}$ & 9 & 0.31 \\
\hline & & 52 & Ccar-19 & $(\mathrm{TG})_{16} \mathrm{CG}(\mathrm{TG})_{3}$ & 4 & 0.30 \\
\hline & South Africa & 43 & Ccar-1 & $(\mathrm{AC})_{22}^{16}$ & 5 & 0.88 \\
\hline & & 43 & Ccar-6 & $(\mathrm{AC})_{17}^{2}$ & 5 & 0.56 \\
\hline & & 43 & Ccar-9 & $(\mathrm{TG})_{23}$ & 12 & 0.41 \\
\hline & & 43 & Ccar-13 & $(\mathrm{TG})_{4}^{2} \operatorname{TT}(\mathrm{TG})_{9} \mathrm{TT}(\mathrm{TG})_{3} \mathrm{TTTT}(\mathrm{TG})_{23}$ & 7 & 0.29 \\
\hline & & 43 & Ccar-19 & $(\mathrm{TG})_{16} \mathrm{CG}(\mathrm{TG})_{3}$ & 3 & 0.38 \\
\hline
\end{tabular}




\begin{tabular}{|c|c|c|c|c|c|c|c|c|}
\hline Species & Sampling locations & $N$ & Locus & Motif & A & $H_{n}$ & $H_{e}$ & Reference \\
\hline Carcharias taurus & northwest Atlantic & $\begin{array}{l}5 \\
5 \\
5 \\
5 \\
5 \\
5 \\
63 \\
63 \\
63 \\
63 \\
63 \\
63 \\
63 \\
12 \\
12 \\
12 \\
12 \\
12 \\
12 \\
75 \\
75 \\
75 \\
75 \\
75 \\
75 \\
24 \\
24 \\
24 \\
24 \\
24 \\
24 \\
7 \\
7 \\
7 \\
7 \\
7 \\
7\end{array}$ & 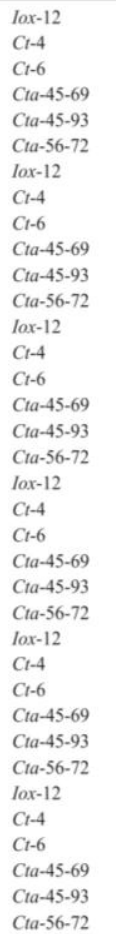 & 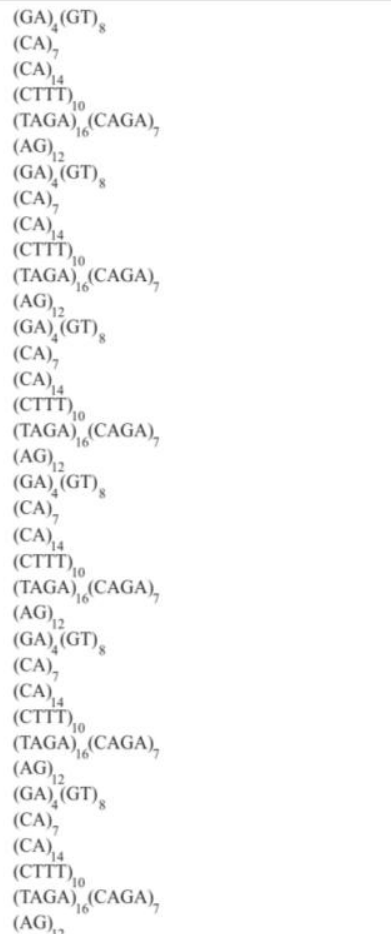 & $\begin{array}{l}4 \\
3 \\
5 \\
4 \\
5 \\
2 \\
5 \\
5 \\
5 \\
12 \\
8 \\
5 \\
1 \\
3 \\
2 \\
11 \\
5 \\
1 \\
8 \\
6 \\
8 \\
10 \\
11 \\
6 \\
5 \\
6 \\
4 \\
10 \\
8 \\
4 \\
1 \\
1 \\
1 \\
2 \\
2 \\
2\end{array}$ & $\begin{array}{l}1 \\
0.40 \\
1 \\
1 \\
1 \\
0.60 \\
0.70 \\
0.68 \\
0.51 \\
0.62 \\
0.81 \\
0.49 \\
0 \\
0.67 \\
0.08 \\
0.75 \\
0.55 \\
0 \\
0.70 \\
0.68 \\
0.64 \\
0.75 \\
0.85 \\
0.55 \\
0.63 \\
0.88 \\
0.75 \\
0.72 \\
0.88 \\
0.64 \\
0 \\
0 \\
0 \\
0.14 \\
0.40 \\
0.71\end{array}$ & $\begin{array}{l}0.75 \\
0.38 \\
0.78 \\
0.68 \\
0.85 \\
0.45 \\
0.65 \\
0.67 \\
0.53 \\
0.58 \\
0.77 \\
0.47 \\
0 \\
0.56 \\
0.08 \\
0.92 \\
0.74 \\
0 \\
0.72 \\
0.65 \\
0.69 \\
0.78 \\
0.80 \\
0.48 \\
0.80 \\
0.75 \\
0.69 \\
0.83 \\
0.86 \\
0.50 \\
0 \\
0 \\
0 \\
0.14 \\
0.35 \\
0.48\end{array}$ & Ahonen et. al. 2009 \\
\hline Squalus acanthias & $\begin{array}{l}\text { Japan } \\
\text { Washington/Oregon } \\
\text { California } \\
\text { New Zealand } \\
\text { Chile } \\
\text { Virginia } \\
\text { Massachussetts } \\
\text { Ireland } \\
\text { Irish Sea } \\
\text { Argentina }\end{array}$ & $\begin{array}{l}49 \\
50 \\
49 \\
46 \\
18 \\
55 \\
48 \\
56 \\
48 \\
48\end{array}$ & $\begin{array}{l}8 \text { loci pooled, } \\
\text { Df-j445 (AC) } \\
\text { Saca-3853 (T } \\
8 \text { loci pooled, } \\
\text { Df-j445 (AC) } \\
\text { Saca-3853 (T } \\
8 \text { loci pooled, } \\
\text { Df-j445 (AC) } \\
\text { Saca-3853 (T }\end{array}$ & 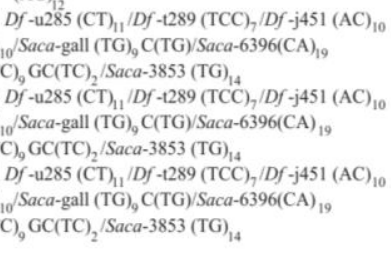 & $\begin{array}{l}6.25 \\
6.88 \\
6.50 \\
7 \\
6.25 \\
8.25 \\
7 \\
8 \\
7.38 \\
7.63\end{array}$ & $\begin{array}{l}0.56 \\
0.55 \\
0.54 \\
0.69 \\
0.64 \\
0.65 \\
0.62 \\
0.62 \\
0.63 \\
0.64\end{array}$ & $\begin{array}{l}0.56 \\
0.55 \\
0.55 \\
0.65 \\
0.64 \\
0.65 \\
0.62 \\
0.62 \\
0.64 \\
0.64\end{array}$ & $\begin{array}{l}\text { Verissimo et. al. } \\
2010\end{array}$ \\
\hline Rhincodon typus & $\begin{array}{l}\text { Global distribution } \\
\text { (Atlantic, Pacific and } \\
\text { Indian ocean) }\end{array}$ & $\begin{array}{l}68 \\
68 \\
68 \\
68 \\
68 \\
68 \\
68 \\
68\end{array}$ & $\begin{array}{l}\text { Rtyp-1 } \\
\text { Rtyp-2 } \\
\text { Rtyp-3 } \\
\text { Rtyp-4 } \\
\text { Rtyp-5 } \\
\text { Rtyp-6 } \\
\text { Rtyp-7 } \\
\text { Rtyp-8 }\end{array}$ & $\begin{array}{l}\text { (TG) })_{2} \mathrm{TC}(\mathrm{TC})_{6} \mathrm{TC}(\mathrm{TG})_{\ldots} .(\mathrm{TG})_{9} . .(\mathrm{TG})_{9} \mathrm{TC}(\mathrm{TG})_{7} \\
(\mathrm{TG})_{3} \mathrm{TC}(\mathrm{TG})_{12} \mathrm{TC}(\mathrm{TG})_{2} \mathrm{TC}(\mathrm{TG})_{8} \mathrm{CG}(\mathrm{TG})_{2} \\
(\mathrm{TG})_{14} .(\mathrm{TG})_{5} . .(\mathrm{TG})_{4} \\
(\mathrm{CT})_{3} . \mathrm{TTTTCTGT}(\mathrm{CT})_{3} \mathrm{GTCT} \\
(\mathrm{CA})_{2_{20}} \\
(\mathrm{GAA})_{41} \\
(\mathrm{CA})_{3} \mathrm{TG}(\mathrm{CA})_{3} \mathrm{TA}(\mathrm{CA})_{3}(\mathrm{CT})_{4} \mathrm{CC}(\mathrm{CA})_{19} \\
(\mathrm{CA})_{3} \mathrm{TGT}(\mathrm{GC})_{4}(\mathrm{CA})_{9} \mathrm{TACA}\end{array}$ & $\begin{array}{l}5 \\
7 \\
4 \\
4 \\
7 \\
34 \\
8 \\
3\end{array}$ & $\begin{array}{l}0.69 \\
0.73 \\
0.80 \\
0.47 \\
0.85 \\
0.57 \\
0.74 \\
0.45\end{array}$ & $\begin{array}{l}0.68 \\
0.74 \\
0.55 \\
0.40 \\
0.87 \\
1 \\
0.83 \\
0.46\end{array}$ & Schmidt et. al. 2009 \\
\hline Galeorhimus galeus & $\begin{array}{l}\text { west of North Island, } \\
\text { New Zealand }\end{array}$ & $\begin{array}{l}40 \\
40 \\
40 \\
40 \\
40 \\
40 \\
40 \\
40 \\
40 \\
47 \\
47 \\
47 \\
47 \\
47 \\
47 \\
47 \\
47 \\
47 \\
30 \\
30 \\
30 \\
30 \\
30 \\
30 \\
30 \\
30 \\
30 \\
43 \\
43 \\
43 \\
43 \\
43 \\
43 \\
43 \\
43 \\
43\end{array}$ & $\begin{array}{l}\text { Ggal-14 } \\
\text { Ggal-15 } \\
\text { Gg-20 } \\
\text { Gg-15 } \\
\text { Gg-22 } \\
\text { Gg-23 } \\
\text { Gg-12 } \\
\text { Gg-7 } \\
\text { Gg-11 } \\
\text { Ggal-14 } \\
\text { Ggal-15 } \\
\text { Gg-20 } \\
\text { Gg-15 } \\
\text { Gg-22 } \\
\text { Gg-23 } \\
\text { Gg-12 } \\
\text { Gg-7 } \\
\text { Gg-11 } \\
\text { Ggal-14 } \\
\text { Ggal-15 } \\
\text { Gg-20 } \\
\text { Gg-15 } \\
\text { Gg-22 } \\
\text { Gg-23 } \\
\text { Gg-12 } \\
\text { Gg-7 } \\
\text { Gg-11 } \\
\text { Ggal-14 } \\
\text { Ggal-15 } \\
\text { Gg-20 } \\
\text { Gg-15 } \\
\text { Gg-22 } \\
\text { Gg-23 } \\
\text { Gg-12 } \\
\text { Gg-7 } \\
\text { Gg-11 }\end{array}$ & 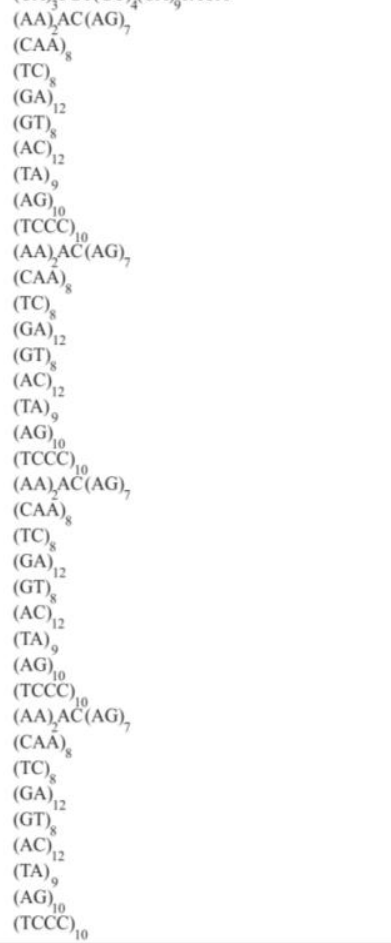 & 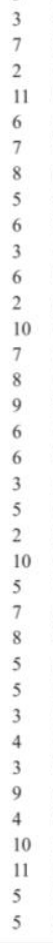 & $\begin{array}{l}0.43 \\
0.32 \\
0.13 \\
0.86 \\
0.61 \\
0.65 \\
0.65 \\
0.68 \\
0.46 \\
0.36 \\
0.55 \\
0.11 \\
0.76 \\
0.58 \\
0.60 \\
0.80 \\
0.65 \\
0.73 \\
0.30 \\
0.57 \\
0.23 \\
0.87 \\
0.30 \\
0.82 \\
0.56 \\
0.50 \\
0.52 \\
0.33 \\
0.49 \\
0.29 \\
0.83 \\
0.44 \\
0.73 \\
0.95 \\
0.63 \\
0.70\end{array}$ & $\begin{array}{l}0.46 \\
0.61 \\
0.29 \\
0.86 \\
0.66 \\
0.75 \\
0.80 \\
0.66 \\
0.74 \\
0.49 \\
0.60 \\
0.15 \\
0.86 \\
0.69 \\
0.80 \\
0.79 \\
0.64 \\
0.58 \\
0.35 \\
0.57 \\
0.21 \\
0.86 \\
0.60 \\
0.81 \\
0.72 \\
0.55 \\
0.50 \\
0.48 \\
0.55 \\
0.32 \\
0.87 \\
0.65 \\
0.75 \\
0.84 \\
0.65 \\
0.64\end{array}$ & This study \\
\hline
\end{tabular}




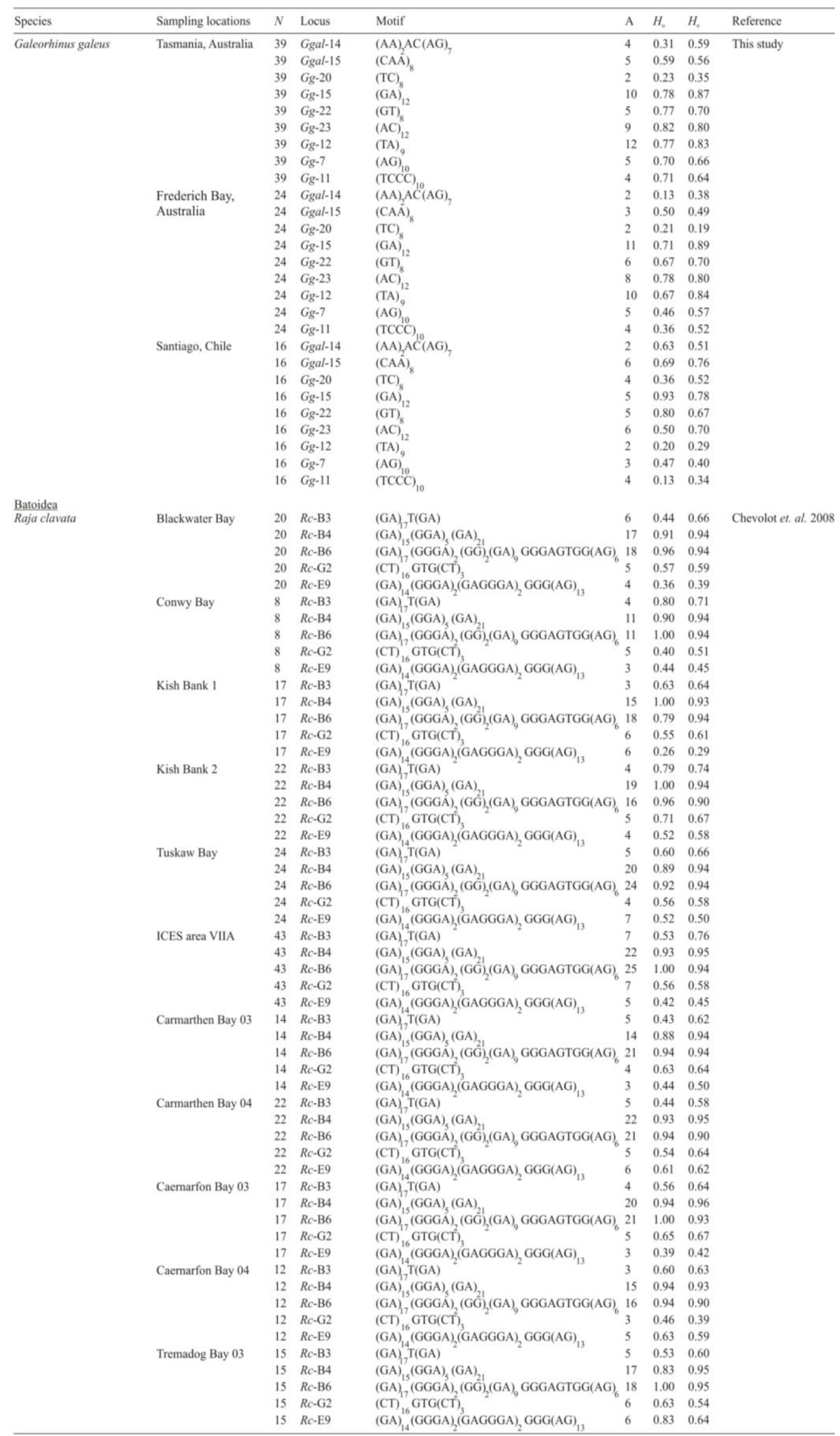




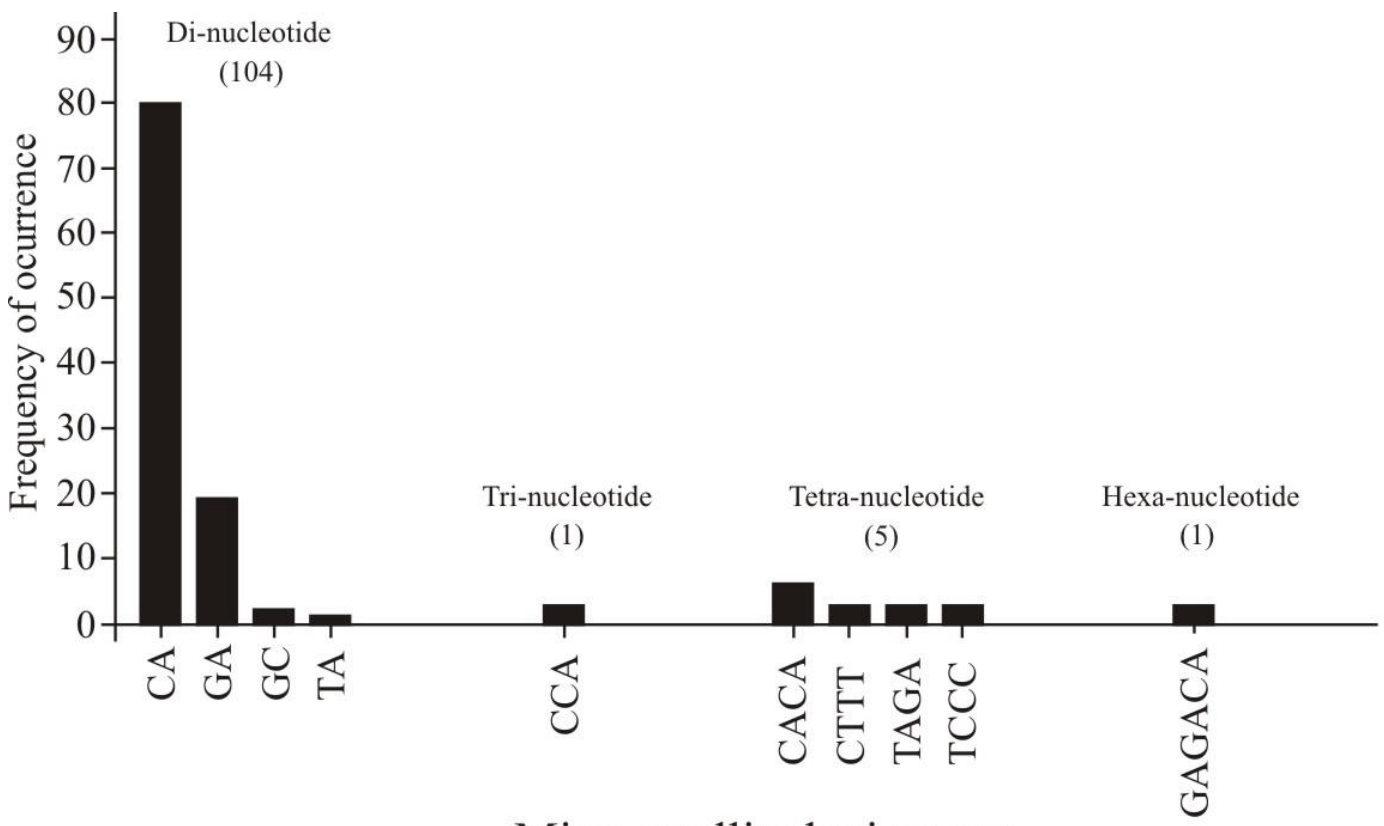

Microsatellite loci repeat

Supplementary Figure 3.1: Distribution of di-nucleotide, tri-, tetra-, and hexa-, repeats units identified in microsatellite population structure studies reported to the date for 15 shark species. The number in brackets is the number of microsatellite repeat for each motif. The $x$ axis is the number of repeat units for each repeat motif and the $y$-axis is the number of microsatellites identified. 


\section{Chapter 4}

\section{Population structure of the school shark (Galeorhinus galeus) in New Zealand, Australian and Chilean waters based on microsatellite DNA markers}

\subsection{Abstract}

DNA microsatellite markers were used to determine the genetic population structure of the school shark, Galeorhinus galeus in Australia, New Zealand and Chile. In total of 239 individuals were genotyped using nine microsatellite loci, which were collected from three sites in New Zealand, three in Australia, and one site in Chile. The number of alleles ranged from 2 for the $G g 20$ locus to 12 for the $G g 12$ locus, and the expected heterozygosities ranged from 0.145 for $G g 20$ to 0.886 for $G g 15$. The analyses of the microsatellites revealed a significant level of differentiation between New Zealand/Australia and Chile. In contrast, there was no evidence of differentiation between sites in New Zealand and sites in Australia. These results, in combination with mtDNA data (see Chapter 2), appear to be consistent with a single panmictic stock in New Zealand and Australian waters. The similarity of the results obtained from the maternally inherited mtDNA and biparental inherited microsatellite loci did not support the suggestion of sex-biased dispersal of G. galeus in the New Zealand/Australia region and it was concluded that females and males had similar patterns of dispersal. 


\subsection{Introduction}

Understanding of patterns of connectivity is important for effective management of a fishery. Despite the commercial importance of several elasmobranch species and concerns about the sustainability of stocks, there have been few studies of stock structure (see details in Heist et al. 2005 and Dudgeon et al. 2012). Most stock connectivity studies have used direct methods (e.g. mark-recapture and other tagging data) to quantify survival and migration rates and determine the patterns of dispersal (Hurst et al. 1999, Kohler \& Turner 2001). However, many of the direct methods can be logistically complicated, because they often require labour-intensive surveys that yield relatively limited data sets, and provide little information about reproductive success (Waples 1998). A combination of direct and indirect methods (e.g. genetic markers) may provide a better understanding of the patterns of population connectivity and structure. Over the last two decades, significant progress has been made to genetic methods used to identify fisheries stocks, estimate effective population size, and identify species for catch and trading surveillance (Dichmont et al. 2011, Dudgeon et al. 2012).

The level of intraspecific genetic connectivity is thought to be positively correlated with dispersal ability. Dispersal patterns tend to be associated with habitat preference (e.g. coastal, pelagic or benthonic), size and reproductive behaviour. In general terms, species of large sharks are considered highly mobile and disperse widely compared to smaller species, which do not tend to be move large distances (Musick et al. 2004, Dudgeon et al. 2012). For example, high levels of gene flow have been reported for Isurus oxyrinchus (Schrey \& Heist 2003), Cetorhinus maximus (Hoezel et al. 2000), and Rhincodon typus (Castro et al. 2007), which are large oceanic species with extensive migratory patterns. In contrast, species less vagile, demersal or benthonic have higher levels of intraspecific genetic differentiation e.g. Raja clavata (Chevolot et al. 2006), Sphyrna lewini (Duncan et al. 2006), Galeorhinus galeus (Chabot \& Allen 2009), and Carcharhinus brachyurus (Benavidez et al. 2011).

A variety of molecular marker types have been used to determine the genetic stock structure of elasmobranchs (Heist 1999, Dudgeon et al. 2012). Early studies were often limited by lack of genetic variability detected using allozyme loci (Smith 
1986, MacDonald 1988, Heist et al. 1995, Ward \& Gardner 1997, 1998) and mitochondrial DNA (mtDNA) restriction fragment-length polymorphism (RFLP) (Heist et al. 1996a, b, Ward \& Gardner 1997, 1998). In recent years mtDNA sequencing and microsatellite DNA markers have been widely used. Microsatellite DNA have been most popular because they are often highly variable, which means they have the statistical power to resolve patterns of gene flow. Microsatellite loci are typically selectively neutral genetic markers, unless they are linked to a gene under selection. The processes of migration, genetic drift and mutation determine allele frequencies in a population (Heist 1999, Dudgeon et al. 2012).

High levels of genetic differentiation in large marine fish populations have been associated with sedentary behaviour, disjunct distributions and/or sex-biased dispersal (Palumbi 1994). The most common pattern of sex-biased dispersal is when one sex is philopatric (i.e. tends to stay or return to its natal site or group to breed) while the other sex is more prone to disperse away from its natal area (Hueter et al. 2005). Different levels of differentiation between the sexes can be detected by comparing the patterns of variation detected using biparentally inherited nuclear markers and the maternally inherited mtDNA. High levels of differentiation at mtDNA and low levels of differentiation at nuclear DNA loci, is often interpreted as resulting from high site fidelity by reproducing females and widely dispersed reproducing males (Hueter et al. 2005). Sex-biased dispersal has been reported in several shark species based on the results of tagging, tracking, and genetic markers (Hueter et al. 2005, Heist 2009). Contrasting patterns of mtDNA and microsatellite loci variation in shark species such as Carcharodon carcharias (Pardini et al. 2001), Isurus oxyrinchus (Schrey \& Heist 2003), Negaprion brevirostris (Schultz et al. 2008), Carcharhinus plumbeus (Portnoy et al. 2010), and Carcharhinus leucas (Karl et al. 2011) have been interpreted as sex-biased dispersal. These studies demonstrated that females typically remained closer to their home territories, when contrasted to males in the population, which roamed over larger distances. If female G. galeus are philopatric to their nursery area and females have formed separate sub-populations, then heavy fishing or habitat destruction could threaten genetically isolated nursery areas and there is a risk of sub-population collapse. Testing for different dispersal behaviour between the sexes is important for the management and conservation of shark fisheries. 
Galeorhinus galeus is a highly mobile species which is widely distributed throughout the temperate waters of New Zealand and Australia. It is characterized by low reproductive potential (i.e. slow-growth, late sexual maturity) that make it highly susceptible to overfishing and habitat degradation. For example, the G. galeus stock in California was collapsed by fisheries (Ripley 1946, Stevens et al. 2001). In Australia during the 1970s, $84 \%$ of the mature biomass was fished out, resulting in an $80 \%$ decline of pups from Port Phillip Bay, Victorian waters (Walker 1998, 1999, Stevens et al. 2001). Habitat degradation in the area such as Geelong and demersal trawling has also put pressure on important nursery areas for G. galeus. Similar patterns were described for Port Sorell and St Helens in Tasmanian waters (Stevens 2005). The limit reproductive potential and the trend of declining population size, result in this species being listed as Vulnerable by the IUCN (Walker et al. 2006).

The high level of gene flow based on the control region (CR) of mtDNA between New Zealand and Australia (see Chapter 2) and the previously published tagging results of G. galeus (Hurst et al. 1999, Walker 2008, Francis 2010), are consistent with the notion of a single panmictic population. This result suggests the lack of any obvious oceanic barriers to migration between New Zealand and Australia. However, the dispersal behaviour of the species also needs to be tested to determine whether there is a cryptic type of population differentiation caused by sexbiased dispersal. The aim of this research was to compare the patterns of variation at nuclear DNA microsatellite loci with mtDNA in G. galeus populations sampled in New Zealand, Australia and Chile in order to describe population connectivity. 


\subsection{Methods}

\subsubsection{Sample collections}

Samples from adult G. galeus were collected from NIWA (National Institute of Water and Atmospheric Research) fisheries surveys and by observers on longline commercial fishing vessels (see Chapter 2 for the collection details). Samples were taken from seven sites within New Zealand (NZ), Australia (AUS) and Chile (CHI). The samples were taken from three NZ sites; east of North Island (ENI), Chatham Rise (CHA) and Solander Islands (SOL) (see Figure 4.1A), three AUS sites; Shoulder (SHO), Tasmania (TAS), and Frederich Bay (FRE) (see Figure 4.1A), and Santiago (SAN) site in CHI (see Figure 4.1B). The sample sizes for each location are listed on Figure 4.1 and Table 4.2.

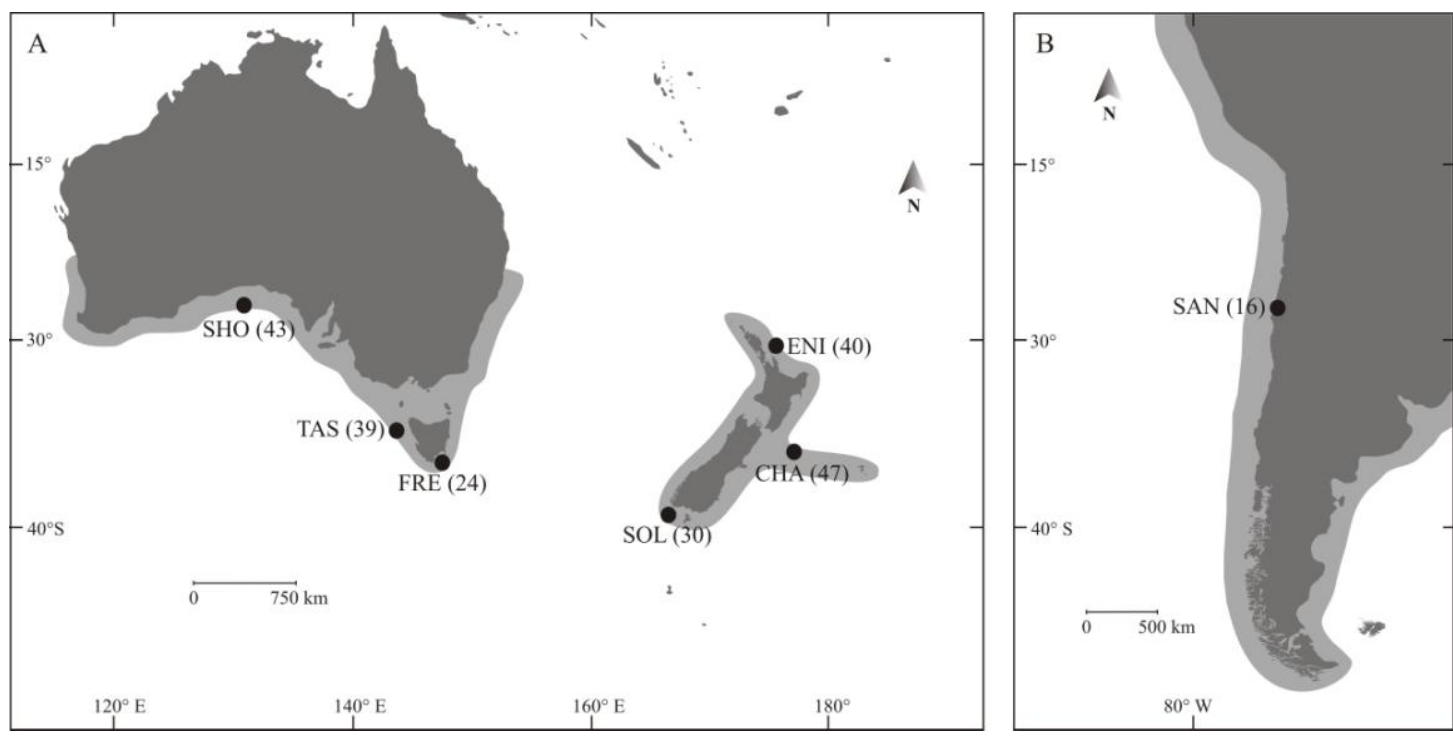

Figure 4.1: Sampling sites, sample sizes (in parentheses), and geographic distribution (shaded area) of adult sharks of G. galeus collected from A. New Zealand, Australia, and B. Chile. The sites are: ENI (east of North Island), CHA (Chatham Rise), SOL (Solander Island), SHO (Shoulder), TAS (West Tasmania), FRE (Frederich Bay), and SAN (Santiago). 


\subsubsection{PCR Amplification and Genotyping}

Total genomic DNA was isolated from tissue samples using a standard phenolchloroform extraction protocol (Sambrook et al. 1989). All samples were genotyped for a total of nine microsatellite loci. Two loci (Ggal14 and Ggal15) were taken from the microsatellite DNA markers that were isolated and developed and reported in Chapter 3. The other seven loci ( $G g 20, G g 15, G g 22, G g 23, G g 12, G g 07$, and $G g 11)$ were taken from Chabot \& Nigenda (2011) and were chosen for their ease of amplification and genotyping, and for their high allelic diversity. Microsatellite loci were PCR amplified separately in $10 \mu 1$ volumes containing $67 \mathrm{mM}$ Tris-HCL pH 8.8, $16 \mathrm{mM}\left(\mathrm{NH}_{4}\right)_{2} \mathrm{SO}_{4}, 2.0 \mathrm{mM} \mathrm{MgCl} 2,0.05 \mu \mathrm{M}$ of forward primer labelled with M-13 tail, $0.2 \mu \mathrm{M}$ of reverse primer, $0.05 \mu \mathrm{M}$ of fluorescently labelled M-13 primers (FAM, VIC, or PET Invitrogen), $0.8 \mathrm{mM}$ of each dNTPs, 0.3 units of BIOTAQ ${ }^{\mathrm{TM}}$ DNA Polymerase (Bioline), and $1 \mu \mathrm{l}$ of template DNA (50-80 ng/ $\mu \mathrm{l}$ DNA concentration). Thermal cycling was performed on a TGradient Thermal Block (Biometra, Goettingen, Germany). Primer pairs and PCR conditions for each locus are detailed in Table 4.1. PCR products from multiple loci were pool-plexed into a single 96-plate well (one set with four loci and another with five loci) based on the combinations recommended by the MULTIPLEX MANAGER 1.0 program (Holleley \& Geerts 2009). Capillary separation of the fluorescently labelled PCR-fragments was conducted on an ABI3730 Genetic Analyser (Massey University Genome Service). The allele sizes were scored using the AUTOBIN program (http://www4.bordeauxaquitaine.inra.fr/biogeco/Ressources/Logiciels/Autobin), which uses an Excel macro to sort and plot the relevant gaps between allele sizes (Guichoux et al. 2011). The alleles were coded as three digit genotypes and the individuals contained missing data at no more than three loci. 
Table 4.1: Primer sequences and PCR conditions of nine microsatellite loci of G. galeus.

\begin{tabular}{|c|c|c|c|c|}
\hline Locus and primer sequences & Repeat & Size (bp) & First cycling conditions & Second cycling conditions \\
\hline $\begin{array}{l}\text { Ggal14F 5'-AGTCAAAGGATGCTCGCAGT-3' } \\
\text { Ggal14R 5'-GGGCGATTATTTGGATGAGA-3' }\end{array}$ & $(\mathrm{AA})_{2} \mathrm{AC}(\mathrm{AG})_{7}$ & $117-125$ & $94^{\circ} 30^{\prime \prime}, 62^{\circ} 45^{\prime \prime}, 72^{\circ} 45^{\prime \prime}, 30$ cycles & $94^{\circ} 30^{\prime \prime}, 53^{\circ} 45^{\prime \prime}, 72^{\circ} 45^{\prime \prime}, 8$ cycles \\
\hline $\begin{array}{l}\text { Ggal15F 5'-GTGACCTCCATCACCGACTT-3' } \\
\text { Ggal15R 5'-GCCTTTGTGAAGAGCCTCAA-3' }\end{array}$ & $(\mathrm{CAA})_{8}$ & $132-159$ & $94^{\circ} 30^{\prime \prime}, 62^{\circ} 45^{\prime \prime}, 72^{\circ} 45^{\prime \prime}, 30$ cycles & $94^{\circ} 30^{\prime \prime}, 53^{\circ} 45^{\prime \prime}, 72^{\circ} 45^{\prime \prime}, 8$ cycles \\
\hline $\begin{array}{l}\text { Gg20F 5'-GACCAAGGGTCATCCAGAC-3' } \\
\text { Gg20R 5'-TCAGCTTGGGCAATTCCAG-3' }\end{array}$ & $(\mathrm{TC})_{8}$ & $197-207$ & $94^{\circ} 30^{\prime \prime}, 59^{\circ} 90^{\prime \prime}, 72^{\circ} 60^{\prime \prime}, 25$ cycles & $94^{\circ} 30^{\prime \prime}, 53^{\circ} 90^{\prime \prime}, 72^{\circ} 60^{\prime \prime}, 20$ cycles \\
\hline $\begin{array}{l}\text { Gg15F 5'-GGCTGAATGGTTTCCCAGC-3' } \\
\text { Gg15R 5'-GCCTCCAACTTAGCATAGCC-3' }\end{array}$ & $(\mathrm{GA})_{12}$ & $152-170$ & 25 cycles & $94^{\circ} 30^{\prime \prime}, 53^{\circ} 90^{\prime \prime}, 72^{\circ} 60^{\prime \prime}, 20$ cycles \\
\hline $\begin{array}{l}\text { Gg22F 5'-TCCTGGGATGGCAACTTCG-3' } \\
\text { Gg22R 5'-AGGCCACCCAACTATCCTG-3' }\end{array}$ & $(\mathrm{GT})_{8}$ & $242-256$ & $94^{\circ} 30^{\prime \prime}, 59^{\circ} 90^{\prime \prime}, 72^{\circ} 60^{\prime \prime}, 25$ cycles & $94^{\circ} 30^{\prime \prime}, 53^{\circ} 90^{\prime \prime}, 72^{\circ} 60^{\prime \prime}, 20$ cycles \\
\hline $\begin{array}{l}\text { Gg23F 5'-ACAGACCACAGGGCATGG-3' } \\
\text { Gg23R 5'-TGCAGAGCAGGCTAGATGG-3' }\end{array}$ & $(\mathrm{AC})_{12}$ & $246-350$ & $94^{\circ} 30^{\prime \prime}, 59^{\circ} 90^{\prime \prime}, 72^{\circ} 60^{\prime \prime}, 25$ cycles & $94^{\circ} 30^{\prime \prime}, 53^{\circ} 90^{\prime \prime}, 72^{\circ} 60^{\prime \prime}, 20$ cycles \\
\hline $\begin{array}{l}\text { Gg12F 5'-TGTCAAACACCATCGCAGG-3' } \\
\text { Gg12R 5'-TGCTCTGAAGTCTACAAGAATGG-3' }\end{array}$ & $(\mathrm{TA})_{9}$ & $284-310$ & $94^{\circ} 30^{\prime \prime}, 59^{\circ} 90^{\prime \prime}, 72^{\circ} 60^{\prime \prime}, 25$ cycles & $94^{\circ} 30^{\prime \prime}, 53^{\circ} 90^{\prime \prime}, 72^{\circ} 60^{\prime \prime}, 20$ cycles \\
\hline $\begin{array}{l}\text { Gg7F 5'-CTGTGGAACAAACTCCAGC-3' } \\
\text { Gg7R } 5 \text { '-AGCTGGTCGAGGTGAATGC-3' }\end{array}$ & $(\mathrm{AG})_{10}$ & $298-312$ & $94^{\circ} 30^{\prime \prime}, 59^{\circ} 90^{\prime \prime}, 72^{\circ} 60^{\prime \prime}, 25$ cycles & $94^{\circ} 30^{\prime \prime}, 53^{\circ} 90^{\prime \prime}, 72^{\circ} 60^{\prime \prime}, 20$ cycles \\
\hline $\begin{array}{l}\text { Gg11F 5'-AAGTTGCACGTTTCCCAGC-3' } \\
\text { Gg11R 5'-TACTGCAGGACCGGTTTCC-3' }\end{array}$ & $(\mathrm{TCCC})_{4}$ & $334-354$ & $94^{\circ} 30^{\prime \prime}, 59^{\circ} 90^{\prime \prime}, 72^{\circ} 60^{\prime \prime}, 25$ cycles & $94^{\circ} 30^{\prime \prime}, 53^{\circ} 90^{\prime \prime}, 72^{\circ} 60^{\prime \prime}, 20$ cycles \\
\hline
\end{tabular}




\subsubsection{Data analyses}

\subsubsection{Genetic diversity}

The dataset was initially checked for the presence of null alleles, allele size shifts and scoring errors due to stuttering, using the software MICRO-CHECKER 2.2.3 (van Oosterhout et al. 2004). Population genetic diversity estimates such as the number of alleles per locus, observed $\left(H_{\mathrm{o}}\right)$ and expected $\left(H_{\mathrm{e}}\right)$ heterozygosities and coefficient of inbreeding $\left(F_{\text {IS }}\right)$ were calculated in ARLEQUIN 3.1.1 (Excoffier et al. 2005). Allelic richness, allele frequencies, and $F_{\text {IS }}$ across loci were estimated in FSAT 2.9.3.2 (Goudet 1995). Allele frequency distributions at each population and loci were generated using GENALEX 6.41 (Peakall \& Smouse 2006). Deviation from Hardy Weinberg equilibrium (HWE) was tested across loci by population, and tests for pairwise Linkage Disequilibrium (LD) between all pair of allele across loci among all populations were calculated in ARLEQUIN 3.1.1. HWE significance was calculated using the exact test (Guo \& Thompson, 1992) with a Markov chain of $10^{6}$ steps and $10^{5}$ dememorization steps. Significance values for $\mathrm{LD}$ were calculated using a likelihood-ratio test (LRT) of 20,000 permutations (Slatkin \& Excoffier, 1996). Sequential Bonferroni corrections (Rice 1989) were applied when appropriate.

A coalescent simulation approach was implemented in LOSITAN (Antao et al. 2008 ) to discover outlier loci. The distribution of loci was simulated based on $F_{\text {ST }}$ values and heterozygosity $\left(H_{\mathrm{e}}\right)$ using the method of Beaumont \& Nichols (1996). Outlier simulations of $10^{4}$ steps were conducted using a 'neutral mean $F_{\mathrm{ST}}$ ' and then by 'forced mean $F_{\mathrm{ST}}$, which improves the reliability of the mean $F_{\mathrm{ST}}$ (Antao et al. 2008). Simulations were run using NZ and AUS samples. Samples from CHI were excluded due to small sample size. The overall $F_{\mathrm{ST}}$ of 0.027 , as expected for neutral markers, was used to estimate the upper and lower $F_{\mathrm{ST}}$ limits at the $0.99 \%$ confidence intervals. Both the stepwise (SMM) and the infinite allele (IAM) mutation models were used in the analysis (Luikart \& Cornuet 1998)

In order to determine whether sufficient sample size was obtained to accurately measure allelic diversity within populations, allele discovery curves were constructed for the two most polymorphic loci using the "PopGenKit" package (Rioux Paquette 2011) in R 2.15 (Ihaka \& Gentleman 1996; R Development Core Team 2011). The function computes the number of sampled alleles for a given constant 
increase in sample size by jackknife resampling at 1,000 replicates. The results were used to plot allele discovery curves within each population.

\subsubsection{Population structure}

Genetic diversity within and among populations and ocean basins (defined as South Pacific Ocean and Tasman Sea) was estimated using an analysis of molecular variance (AMOVA, Excoffier et al. 1992) implemented in ARLEQUIN 3.1.1 (Excoffier et al. 1995). Spatial analysis was undertaken to test the hypothesis of panmixia among sites (NZ, AUS and CHI). The variance components and $\Phi$-statistic values were partitioned at three levels of hierarchical subdivision: among ocean basins, within populations, and the interaction of both (among + within). The genetic distance matrix for AMOVA was estimated by pairwise differences and the significance levels of the variance components and $\Phi$-statistics values were tested by 20,000 nonparametric permutations (Excoffier et al. 1995).

Levels of genetic differentiation among populations were estimated by four approaches. a) Testing for differences of allele frequencies distribution between all pairs of populations was conducted in GENEPOP 4.0.10 (Raymond \& Rousset 1995). Unbiased $P$ values were computed for each population pair at each locus using an Exact $G$ test (Goudet et al. 1996), and $P$ values for each population pair across all loci were also calculated by Fisher's exact probability test (Raymond \& Rousset 1995). Markov chain parameters consist of a number of $10^{4}$ dememorization steps, with $10^{3}$ batches and $10^{4}$ iterations per batch. b) Estimating the Wright's $F_{\text {ST }}(\theta$ estimator, Weir \& Cockerham (1984) was computing in FSTAT 2.9.3.2 (Goudet, 1995). The significance values for $\theta$ were calculated by a 1,000 replicate bootstrap procedure over all loci using 99\% confidence intervals. A sequential Bonferroni correction was used to minimise the chances of a type I error of significance over multiple tests (Rice 1989). c) Estimating the Slatkin's $R_{\mathrm{ST}}$ (Slatkin 1995) that considers the square size differences between alleles and it is more appropriate for microsatellite loci that evolve via step-wise mutations. The $R_{\mathrm{ST}}$ estimator was obtained using ARLEQUIN 3.1.1 (Excoffier et al. 1995). (d) Calculating the $D_{\mathrm{ST}}$ estimator (Jost 2008) using SMOGD 1.2.5 (Crawford 2010) with 1,000 bootstrap iterations. The approximate harmonic mean $(H)$ was calculated from the mean and variance across loci (SMOGD 
website www.ngcrawford.com/django/jost/). This statistic is more robust than $F_{\mathrm{ST}}$ due to the bias introduced by differences in diversity among populations, and also more appropriate for highly polymorphic markers (Jost 2008).

POWSIM 4.1 (Ryman \& Palm 2006) was used to assess the statistical power of the sampled loci under different combinations of samples sizes, number of loci and alleles, and allele frequencies. The statistical power and alpha values were assessed using the Chi-squared and Fisher's exact tests under a specified level of population divergence $\left(F_{\mathrm{ST}}\right)$. Two set of simulations with 20,000 replications were run assuming a) a base population with the average observed allele frequencies for the microsatellite loci, and b) a division of seven populations with the sample sizes used in the present study (see number of sharks by population, Table 4.2). Different combinations of $N_{\mathrm{ef}}$ (effective population size) and $t$ (generations of drift) were simulated $\left(N_{\mathrm{e} f} / t\right)$ corresponding to 500/50 and 2000/205 respectively.

A test for a pattern of genetic isolation-by-distance (IBD) was conducted using linearised $F_{\mathrm{ST}}$ values that were plotted against the natural logarithm of geographic distance (kilometres) between all possible pairs of NZ and AUS sites. Geographic distances were estimated by the ruler option in Google Earth v.6.2.2 (Google Inc.). The distance calculations consist of the shortest path, by sea, between every pair of sample sites. Significance was evaluated for the set of pairwise combinations using a Mantel's test implemented in ARLEQUIN 3.1.1 using 20,000 permutations.

\subsubsection{Relationship among populations and Bayesian clustering}

The genetic relationships among populations were examined using a Principal Component Analysis (PCA) based upon the pairwise $F_{\mathrm{ST}}$ values using the program PCA-GEN 1.2.1 (Goudet 1999). Simulations of 15,000 randomizations were performed to evaluate the reliability of the results using two schemes: a) all sampling sites, and b) only the NZ and AUS sites (excluding SAN, Chile). The genetic differences among all individuals from each population were also displayed by Factorial Component Analysis (FCA) using GENETIX 4.05 (Belkhir 2000). Additionally, a cluster analysis was used to investigate the genetic relationships among populations using POPULATIONS 1.2.32 (Langella 2002). The genetic 
distances among populations were estimated using $D a$ (genetic distance; Nei et al., 1983) and the neighbor-joining algorithm was used to construct a dendogram with the resulting distance matrix with and without 1,000 bootstrapped replicates of the dataset.

A Bayesian approach was used to test for population genetic structure using STRUCTURE 2.3.3 (Pritchard et al. 2000, Falush et al. 2003). This program analyses allele frequencies across multiple loci and assigns multi-locus genotypes or individuals to a 'population' cluster ( $K=$ the number of genetically distinct clusters) by minimizing Hardy-Weinberg and linkage disequilibrium in the genotype data. A Markov chain Monte Carlo (MCMC) simulation was run for $10^{7}$ iterations with a burn-in period of 400,000 steps (Falush et al. 2003). Multiple runs were performed to evaluate the reliability of the results under admixture and non-admixture and locprior models because it performs better than other models for detecting genetic structure even in situations of low levels of genetic divergence or a limited number of loci (Hubisz et al. 2009). Two ranges of $K$ values were tested depending on the number of population included in the analysis: (1) using all sample sites ( $K$ ranged from 1 to 7 ) and (2) only the NZ and AUS sample sites ( $K$ ranged from 1 to 6). STRUCTURE HARVESTER 0.6.92 (Dent \& Bridgett 2012) was used to estimate the optimum number of $K$ using the Log probability of data ( $L(K)$; Pritchard et al. 2000). However, if the migration rates are not equal between populations, $K$ may be incorrectly estimated. Thus, Delta $K$ estimator ( $\Delta K$; Evanno et al. 2005) was estimated. Delta $K$ was more accurate than Log probability in estimating the true $K$ because it is based on the rate of change in the log probability of data between successive $K$-values. Individuals were assumed to have been correctly assigned to a population when their $q$ value (i.e. its posterior probability of belonging to original population) was at least $80 \%$ for the population (Pritchard et al. 2000).

\subsubsection{Mutation-drift and migration-drift equilibrium}

Three approaches were used to investigate whether there was evidence of a recent population size contraction. First, BOTTLENECK 1.2.02 (Pyri et al. 1999) was used to test for an excess of heterozygotes. Expected heterozygosity $\left(H_{\mathrm{e}}\right)$ was estimated from the observed alleles $(k)$ at each locus and a distribution of heterozygosity was 
generated based on the simulated coalescening of nine loci under the Stepwise Mutational Model (SMM), Infinite Alleles Model (IAM), and the Two-Phase Model (TPM). The interpretation of results focused on the TPM of mutation as it is recommended for microsatellite loci due to a better fit with observed allele frequency data than the IAM and SMM (Di Rienzo et al. 1994, Piry et al. 1999). The TPM option was set at 95\% single-step mutations and 5\% multiple-step mutations (Cornuet \& Luikart 1996, Piry et al. 1999). The average expected equilibrium heterozygosity $\left(H_{\mathrm{eq}}\right)$ for each locus was compared to Hardy-Weinberg heterozygosity $\left(H_{\mathrm{e}}\right)$ and used to assess whether there was a heterozygote excess or deficit at each locus using a Sign test, standardized differences test, and Wilcoxon's test based on 1000 replications. The second approach used BOTTLENECK 1.2.02 to test for a mode shift of the allele frequency distribution from an L-shaped distribution that develops when the population is in a mutation-drift balance. Thirdly, a population size contraction signature was tested using $M$-RATIO method developed by Garza \& Williamson (2001). This approach calculates $M$, which is the ratio of total number of alleles $(k)$ compared to the range of allele sizes $(r)$. The program M_P_VAL 100.0 (Garza \& Williamson 2001) was used to calculate $M$-RATIO $(M)$ for each population and contrast the observed values of $M$ to a distribution of $M$ values calculated from simulated populations assumed to be at mutation-drift equilibrium. The critical values of $M$ were determined by simulations described in Garza \& Williamson 2001. The simulations require three input parameters $\left(\theta, P_{\mathrm{g}}, \Delta_{\mathrm{g}}\right)$. The parameter theta, $\theta=4 N_{\mathrm{ef}} \mu$, where $N_{\text {ef }}=$ effective population size and $\mu=$ mutation rate. Calculation of effective population size was obtained in ARLEQUIN 3.1.1 for each population. Theta $(\theta)$ was estimated from the expected homozygosity $\left(\mathrm{Hom}_{E}\right)$. Assuming that the populations are in mutation-drift equilibrium, $\theta=\left(1-H o m_{\mathrm{E}}\right) / \operatorname{Hom}_{\mathrm{E}}$, where $\left.\operatorname{Hom}_{\mathrm{E}}=1-H_{\mathrm{E}}\right)$, and $H_{\mathrm{E}}$ is the expected heterozygosity. The mutation rate $(\mu)$ was set at $1 \times 10^{-3}$ mutations/generation/locus (Weber \& Wong 1993, DeWoody \& Avise 2000). For both parameters the percentage of mutations larger than a single step $\left(P_{\mathrm{g}}=0.10\right)$ and the mean size of mutations larger than a single step $\left(\Delta_{\mathrm{g}}=3.5\right)$ were chosen following the recommendation of Garza \& Williamson (2001). Each set of simulations was run for 10,000 iterations. 


\subsection{Results}

\subsubsection{Genetic diversity}

Two hundred and thirty nine G. galeus samples from New Zealand, Australia and Chilean sites were genotyped for nine microsatellite loci. Complete genotypes for all loci were obtained for 197 individuals and 42 individuals had missing data of up to three loci. No null alleles, allele size shifts, or error scoring were detected using MICRO-CHECKER 2.2.3 (at the 95\% confidence interval). The summary statistics for genetic diversity at nine microsatellite loci are presented in Table 4.2. The least polymorphic loci were Gg20 and Ggal14, which had between 2 and 3 alleles for NZ and AUS sites, and the most polymorphic loci were $G g 15$ and $G g 12$ for NZ and AUS, which had between 9-11 and 8-12 alleles respectively. When allelic richness was averaged $\left(R_{s}\right)$ across all loci it was greatest in the NZ and AUS sites (4.657-5.050), when compared to the $\mathrm{CHI}$ site (3.984). The observed heterozygosity $\left(H_{\mathrm{o}}\right)$ ranged between 0.111 (CHA, NZ) for $G g 20$, to 0.863 (SOL, NZ) for $G g 15$. The expected heterozygosity $\left(H_{\mathrm{e}}\right)$ varied between $0.145(\mathrm{CHA}, \mathrm{NZ})$ for $G g 20$, to 0.886 (FRE, AUS) for $G g 15$.

After correction for multiple tests, only four of sixty three values showed significant departures from HWE $(P<0.0008)$ across nine microsatellite loci (Table 4.2). There was a significant level of heterozygote deficiency at the Ggal 14 in TAS (AUS), Gg15 in the CHA (NZ), Gg22 in the FRE (AUS) and ENI (NZ) populations, as well as at the Gg11 in the SOL (NZ) and SHO (AUS) populations (Table 4.2). These results support the hypothesis of the presence of null alleles at these loci. In addition to heterozygote deficiencies, only the $G g 22$ and $G g 11$ loci showed a significant level of LD $(P<0.002)$ (data not shown).

In addition, the allele frequency distributions showed that each locus had one relatively common allele that was shared among all populations (Figure 4.2, 4.3, 4.4). Private alleles were found at seven loci (Ggal14, Ggal15, Gg20, Gg22, Gg12, Gg07, and $G g 11$ ), and these were typically at low frequencies. All allele discovery curves reached a plateau when the cumulative allelic richness was plotted against the individuals genotyped (Figure 4.5). The loci Gg15 and Gg12 were found to be the most polymorphic for NZ and AUS, while the loci Ggal15 and Gg23 were for Chile. 
Table 4.2: Summary of nine microsatellite loci for each G. galeus population. Population in New Zealand (NZ), Australia (AUS) and Chile (CHI) are as follows: ENI = east of North Island, $\mathrm{CHA}=$ Chatham Rise, $\mathrm{SOL}=$ Solander Islands, $\mathrm{SHO}=$ Shoulder, TAS $=$ West Tasmania, FRE $=$ Frederich Bay, and SAN $=$ Santiago. Parameters are as follows: $n$, sample sizes; $R_{S}$, allelic richness, $H_{\mathrm{o}}$, observed microsatellite heterozygosity; $H_{\mathrm{e}}$, expected microsatellite heterozygosity; $F_{\text {is }}$, microsatellite inbreeding coefficient [negative values = heterozygote excess and positive values $=$ heterozygote deficiency; AAL $=$ Average across loci; values marked with $*$ deviate from Hardy-Weinberg expectations nominally after sequential Bonferroni correction (alpha $=0.05 / 63=0.0008$ ).

\begin{tabular}{|c|c|c|c|c|c|c|c|c|c|c|c|}
\hline Population & Parameter & Ggal14 & Ggal15 & $G g 20$ & $G g 15$ & $G g 22$ & $G g 23$ & $G g 12$ & Gg7 & $G g 11$ & AAL \\
\hline \multirow{5}{*}{$\begin{array}{l}\text { ENI (NZ) } \\
n=40\end{array}$} & Alleles & 3 & 7 & 2 & 11 & 6 & 7 & 8 & 5 & 6 & 6 \\
\hline & $R_{s}$ & 2.547 & 4.524 & 1.998 & 8.510 & 4.480 & 6.037 & 7.155 & 4.459 & 5.133 & 4.983 \\
\hline & $H_{\mathrm{o}}$ & 0.425 & 0.316 & 0.132 & 0.861 & 0.605 & 0.649 & 0.649 & 0.675 & 0.462 & 0.530 \\
\hline & $H_{\mathrm{e}}$ & 0.460 & 0.610 & 0.287 & 0.863 & 0.659 & 0.746 & 0.799 & 0.664 & 0.743 & 0.648 \\
\hline & $F_{\text {is }}$ & 0.076 & $0.486^{*}$ & 0.545 & 0.002 & 0.082 & 0.132 & 0.190 & -0.017 & $0.382^{*}$ & 0.183 \\
\hline \multirow{5}{*}{$\begin{array}{l}\text { CHA (NZ) } \\
n=47\end{array}$} & Alleles & 3 & 6 & 2 & 10 & 7 & 8 & 9 & 6 & 6 & 6 \\
\hline & $R_{s}$ & 2.479 & 4.192 & 1.917 & 8.221 & 4.308 & 6.437 & 7.477 & 4.694 & 4.290 & 4.891 \\
\hline & $H_{\mathrm{o}}$ & 0.362 & 0.553 & 0.111 & 0.761 & 0.575 & 0.600 & 0.804 & 0.652 & 0.733 & 0.572 \\
\hline & $H_{\mathrm{e}}$ & 0.488 & 0.596 & 0.145 & 0.859 & 0.686 & 0.800 & 0.787 & 0.636 & 0.580 & 0.619 \\
\hline & $F_{\text {is }}$ & 0.260 & 0.072 & 0.236 & 0.115 & 0.164 & 0.252 & -0.022 & -0.025 & -0.269 & 0.077 \\
\hline \multirow{5}{*}{$\begin{array}{l}\text { SOL (NZ) } \\
n=30\end{array}$} & Alleles & 3 & 5 & 2 & 10 & 5 & 7 & 8 & 5 & 5 & 6 \\
\hline & $R_{s}$ & 2.433 & 3.942 & 1.986 & 8.414 & 3.840 & 6.379 & 6.993 & 3.646 & 4.276 & 4.657 \\
\hline & $H_{\mathrm{o}}$ & 0.300 & 0.567 & 0.233 & 0.867 & 0.300 & 0.821 & 0.556 & 0.500 & 0.517 & 0.518 \\
\hline & $H_{\mathrm{e}}$ & 0.352 & 0.574 & 0.210 & 0.861 & 0.604 & 0.806 & 0.716 & 0.554 & 0.499 & 0.575 \\
\hline & $F_{\text {is }}$ & 0.150 & 0.012 & -0.115 & -0.007 & $0.508^{*}$ & -0.020 & 0.228 & 0.099 & -0.037 & 0.101 \\
\hline \multirow{5}{*}{$\begin{array}{l}\text { SHO (AUS) } \\
n=43\end{array}$} & Alleles & 3 & 4 & 3 & 9 & 4 & 10 & 11 & 5 & 5 & 6 \\
\hline & $R_{s}$ & 2.900 & 3.073 & 2.308 & 8.067 & 3.515 & 7.304 & 7.881 & 4.565 & 3.814 & 4.825 \\
\hline & $H_{\mathrm{o}}$ & 0.333 & 0.489 & 0.286 & 0.833 & 0.442 & 0.731 & 0.950 & 0.628 & 0.697 & 0.597 \\
\hline & $H_{\mathrm{e}}$ & 0.478 & 0.552 & 0.316 & 0.867 & 0.650 & 0.746 & 0.838 & 0.647 & 0.639 & 0.645 \\
\hline & $F_{\text {is }}$ & 0.305 & 0.117 & 0.098 & 0.039 & $0.322 *$ & 0.019 & -0.135 & 0.030 & -0.093 & 0.060 \\
\hline \multirow{5}{*}{$\begin{array}{l}\text { TAS (AUS) } \\
n=39\end{array}$} & Alleles & 4 & 5 & 2 & 10 & 5 & 9 & 12 & 5 & 4 & 6 \\
\hline & $R_{s}$ & 3.301 & 3.477 & 2.000 & 8.618 & 4.117 & 7.096 & 8.659 & 4.618 & 3.566 & 5.050 \\
\hline & $H_{\mathrm{o}}$ & 0.306 & 0.590 & 0.231 & 0.778 & 0.769 & 0.821 & 0.769 & 0.692 & 0.711 & 0.618 \\
\hline & $H_{\mathrm{e}}$ & 0.589 & 0.562 & 0.345 & 0.871 & 0.700 & 0.792 & 0.833 & 0.662 & 0.638 & 0.664 \\
\hline & $F_{\text {is }}$ & $0.485^{*}$ & -0.050 & 0.335 & 0.108 & -0.101 & -0.037 & 0.077 & -0.046 & -0.116 & 0.070 \\
\hline \multirow{5}{*}{$\begin{array}{l}\text { FRE (AUS) } \\
n=24\end{array}$} & Alleles & 2 & 3 & 2 & 11 & 6 & 8 & 10 & 5 & 4 & 6 \\
\hline & $R_{s}$ & 1.911 & 2.542 & 1.985 & 9.491 & 4.625 & 7.350 & 8.362 & 4.321 & 3.777 & 4.929 \\
\hline & $H_{\mathrm{o}}$ & 0.125 & 0.500 & 0.208 & 0.708 & 0.667 & 0.783 & 0.667 & 0.458 & 0.364 & 0.498 \\
\hline & $H_{\mathrm{e}}$ & 0.120 & 0.494 & 0.191 & 0.886 & 0.707 & 0.800 & 0.840 & 0.565 & 0.519 & 0.569 \\
\hline & $F_{\text {is }}$ & -0.046 & -0.013 & -0.095 & 0.204 & 0.058 & 0.022 & 0.209 & 0.192 & $0.304^{*}$ & 0.127 \\
\hline \multirow{5}{*}{$\begin{array}{l}\text { SAN (CHI) } \\
n=16\end{array}$} & Alleles & 2 & 6 & 4 & 5 & 5 & 6 & 2 & 3 & 4 & 4 \\
\hline & $R_{s}$ & 2.000 & 6.000 & 3.857 & 4.867 & 4.733 & 5.624 & 2.000 & 2.999 & 3.778 & 3.984 \\
\hline & $H_{\mathrm{o}}$ & 0.625 & 0.692 & 0.357 & 0.933 & 0.800 & 0.500 & 0.200 & 0.467 & 0.125 & 0.493 \\
\hline & $H_{\mathrm{e}}$ & 0.508 & 0.757 & 0.521 & 0.779 & 0.671 & 0.704 & 0.287 & 0.398 & 0.337 & 0.553 \\
\hline & $F_{\text {is }}$ & -0.240 & 0.089 & 0.323 & -0.206 & -0.200 & 0.296 & 0.311 & -0.181 & 0.636 & 0.113 \\
\hline Total & \# Sharks & 235 & 234 & 232 & 229 & 236 & 229 & 228 & 235 & 232 & 232 \\
\hline
\end{tabular}



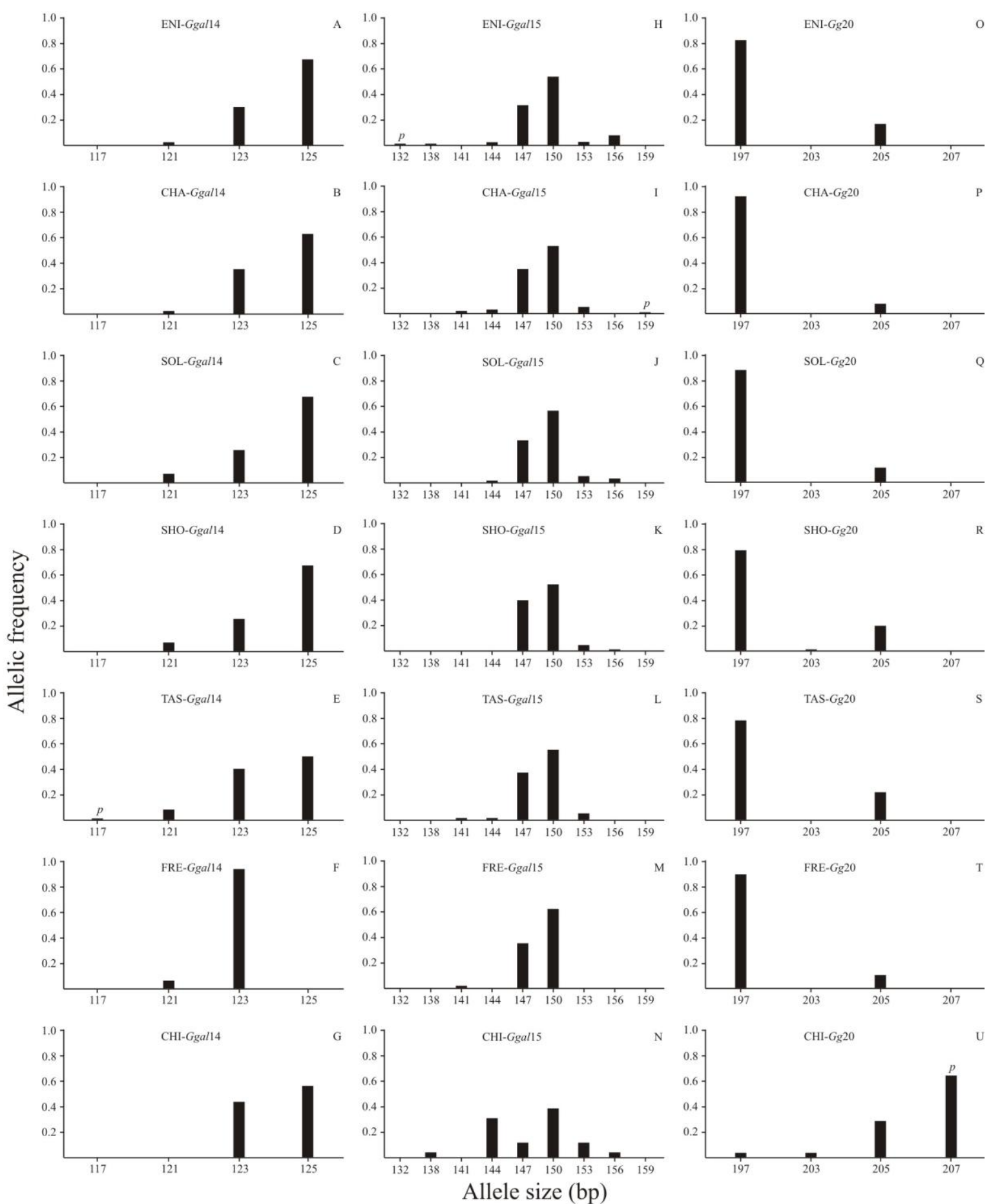

Figure 4.2: Allelic frequency distribution for the microsatellite loci Ggal14, Ggal15, and $G g 20$ across all populations of G. galeus. Allele sizes are given in base pairs of PCR products. The letter " $p$ " represents private alleles found in one population. 

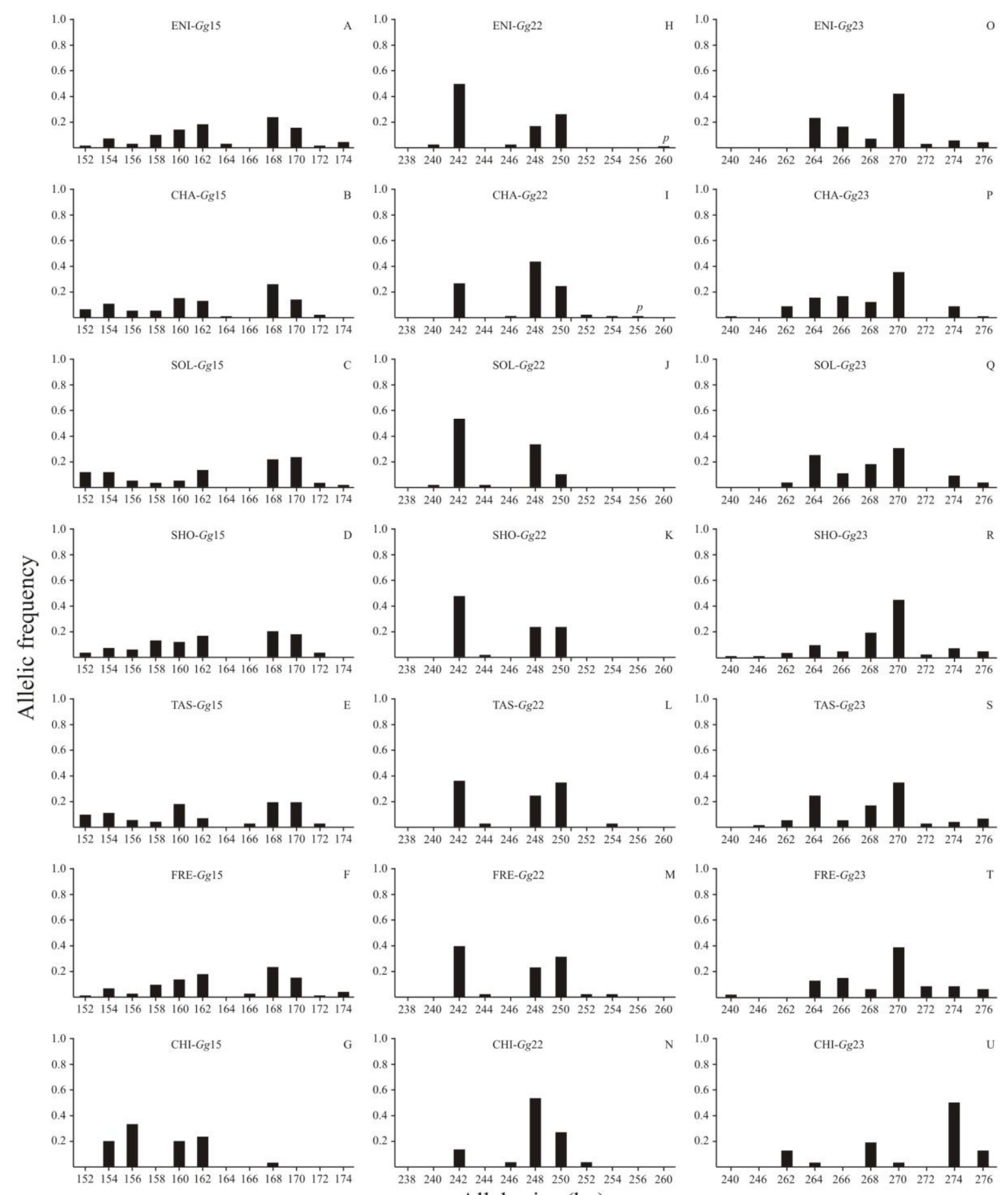

Allele size (bp)

Figure 4.3: Allelic frequency distribution for the microsatellite loci $G g 15, G g 22$, and $G g 23$ across all populations of G. galeus. Allele sizes are given in base pairs of PCR products. The letter " $p$ " represents private alleles found in one population. 

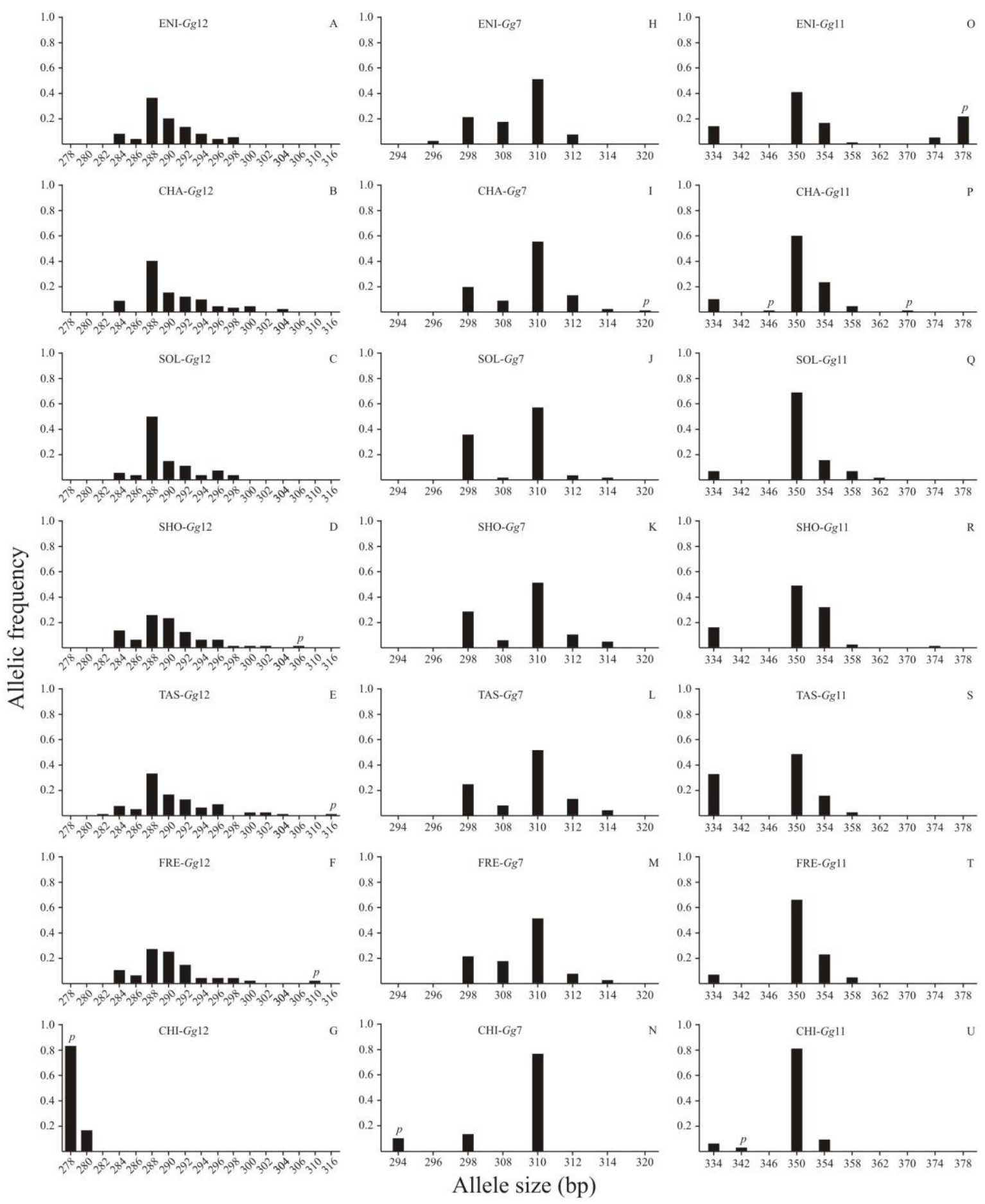

Figure 4.4: Allelic frequency distribution for the microsatellite loci $G g 12, G g 07$, and $G g 11$ across all populations of G. galeus. Allele sizes are given in base pairs of PCR products. The letter " $p$ " represents private alleles found in one population. 

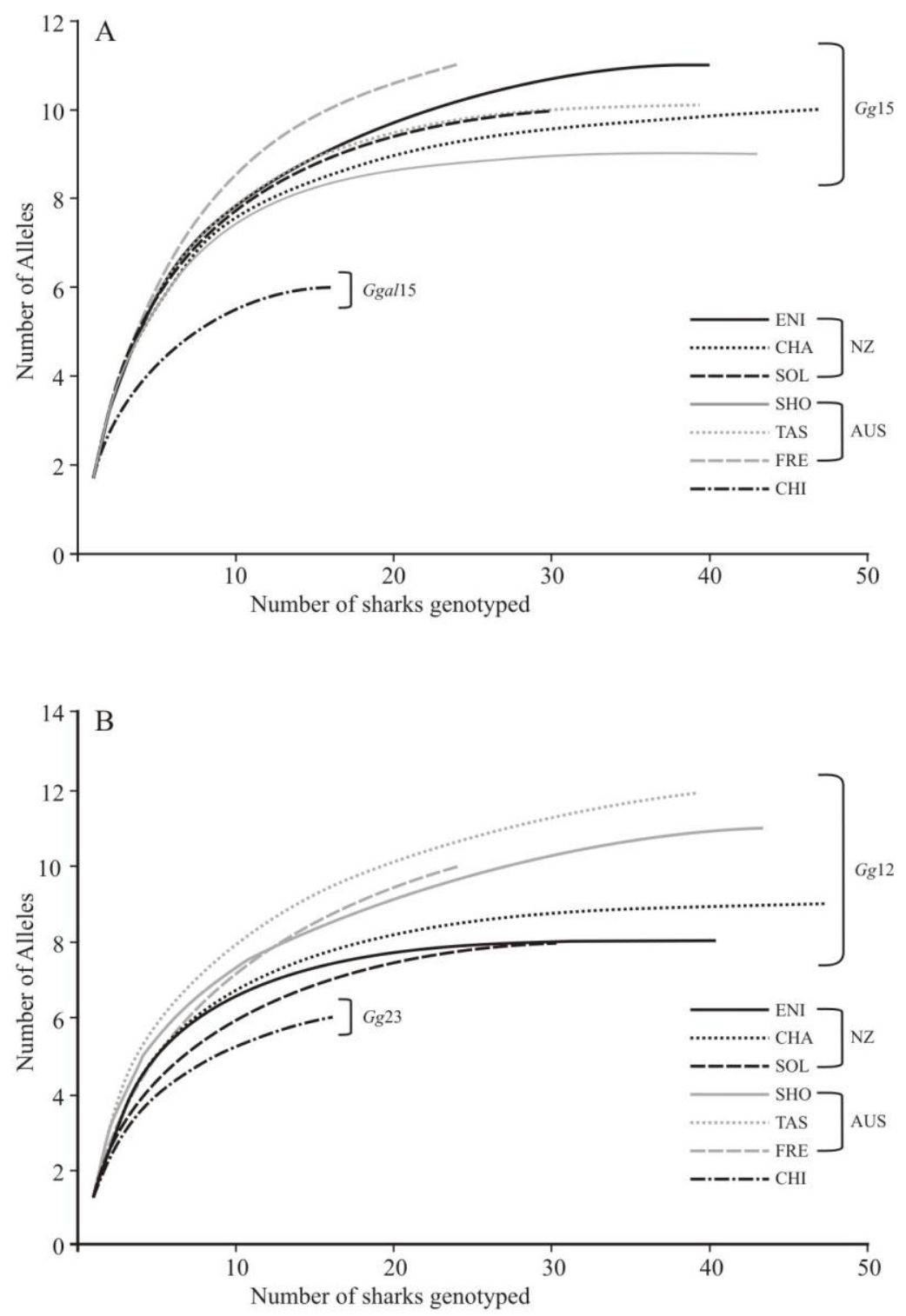

Figure 4.5: Allele discovery curves for the most polymorphic loci. A. Gg15 (NZ and AUS) and Ggal15 (CHI) and B. Gg12 (NZ and AUS) and Gg23 (CHI). 


\subsubsection{Population structure}

The AMOVA analysis showed evidence of population differentiation with significant components of variance within and between ocean basins $\left(F_{\mathrm{CT}}=0.05, \% V=5.45, P\right.$ $=0.0419$ and $F_{\mathrm{SC}}=0.01, \% V=0.79, P=0.0025$, Table 4.3A). However, more than $90 \%$ of the partitioned genetic variation was found within populations $\left(F_{\mathrm{ST}}=0.06, \% \mathrm{~V}\right.$ $=93.76, P<0.0001)$. The second spatial AMOVA was run for NZ and AUS (excluding $\mathrm{CHI}$ ), which revealed non-significant differentiation among groups across Tasman Sea $\left(\Phi_{C T}=0.0005, p=0.39\right)$. The Exact tests of allele frequency distributions indicated regional differentiation between NZ/AUS and CHI at most of the loci $(P<$ 0.001; Table 4.3). Significant differences were detected between NZ and AUS that were driven by one or two loci (see superscript numbers; Table 4.3A). The Ggal14 locus at the FRE site was significantly different from all other sample sites because of an absence of the $125 \mathrm{bp}$ allele from the FRE site (see allelic distribution Figure 4.2F). The Ggal14 locus was also an outlier in the LOSITAN simulation. This locus was removed from the subsequent analyses.

The pairwise $F_{\mathrm{ST}}$ analysis showed a significant level of genetic differentiation between collection sites in NZ/AUS, compared with CHI. However, there was no significant genetic differentiation among sample sites within and between the NZ and AUS regions $\left(F_{\mathrm{ST}}\right.$ values below diagonal table, Table 4.3B). A similar pattern was seen in values of $D_{\mathrm{ST}}$, with relatively higher values than $F_{\mathrm{ST}}$ when $\mathrm{CHI}$ was compared to all other sites and lower values of $D_{\mathrm{ST}}$ when comparison was made within and between NZ and AUS (Table 4.3B). Considering just the NZ and AUS sample sites, the Mantel test did not show a significant relationship between genetic and geographic distance $\left(R^{2}=0.008 P=0.463\right)$.

The POWSIN analysis indicated that the eight loci from all seven sample sites showed that the samples sizes were capable of detecting a true $F_{\mathrm{ST}}$ value, estimated to be as low as 0.05 with a probability of $100 \%$ (both Fisher's exact test and chi-square, those values are not shown) for the two different combination of $N_{\mathrm{ef}} / t$ simulated. 
Table 4.3: A. AMOVA results for global panmixia, across ocean basins (New Zealand, Australia, and Chile) based on eight microsatellite loci. The asterisk (*) represents significant values at $P<0.05$, and ** significant values at $P<0.001$. The SSD is the sum of squares, VC is variance components, and $\% V$ is the percentage of total variation. B. Below the diagonal are the pairwise $F_{\mathrm{ST}}$ values (Weir \& Cockerham 1984), in parentheses the $R_{\mathrm{ST}}$ values (Slatkin 1995), and in italics the $D_{\mathrm{ST}}$ values (Jost 2008) for eight microsatellite loci (excluding Ggal14). Above the diagonal is the number of loci, name of loci (superscript), and $P$ values for Exact $G$ tests from nine microsatellite loci (including Ggal14) between New Zealand, Australia and Chile populations. Significant $P$ values for the pairwise $F_{\mathrm{ST}}$ and Exact tests are represented by $* P<0.001$.

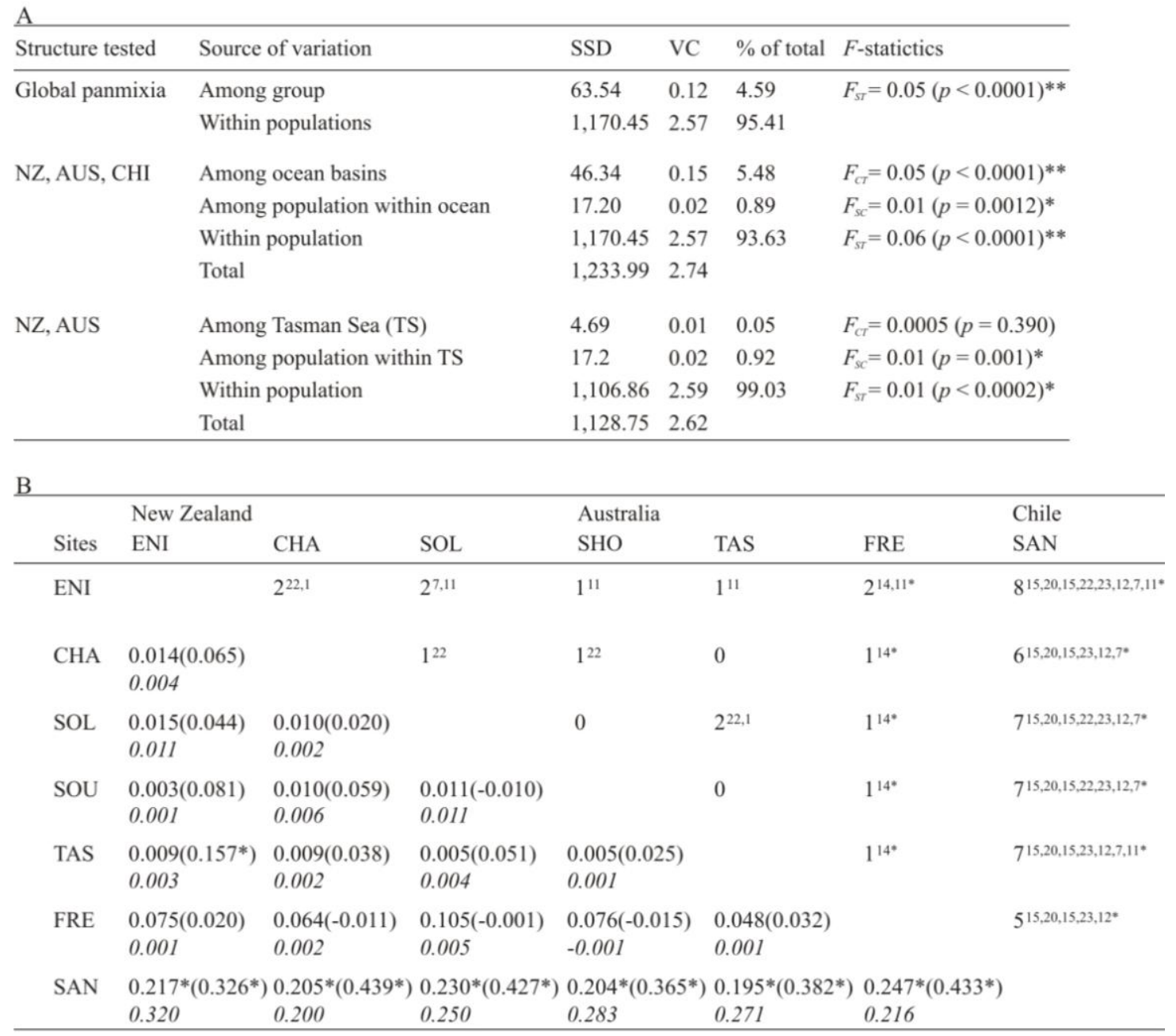




\subsubsection{Relationship among populations and Bayesian clustering}

The PCA also showed that the CHI site was different from all of the NZ and AUS sites (PCA 1: $F_{\mathrm{ST}}=0.037,67.84 \%$ of total inertia, $P<0.001$, Figure $4.6 \mathrm{~A}$ ). When the SAN sample site (CHI) was excluded, the PCA analysis of the NZ and AUS sites suggested some genetic differentiation, but the axis separation of these groups was not significant (Figure 4.6B). The FCA showed a similar result at the individual level instead of population (PCA). FCA showed a few individuals from the ENI (NZ) site outside of the main cluster of individuals from the other NZ and AUS sites (see Figure 4.7). The neighbor-joining dendogram showed an almost star-shaped phylogeny with very little support for any grouping within and between NZ and AUS sites (Figure $4.8)$.

The STRUCTURE analysis distinguished three distinct clusters with the NZ, AUS, and CHI sample collections using the $L(K)$ approach for admixture and nonadmixture models (the highest for $K=3$; Figure 4.9). Two of the clusters were found in NZ and AUS: cluster one was comprised of ENI and cluster two was comprised of CHA, SOL, SHO, TAS, and FRE. A distinct third cluster was formed by the SAN samples (Figure 4.9). Most of the individuals grouped in cluster two and three were assigned to the same cluster (shown in gray and black bar graphs in Figure 4.9), while individuals collected from ENI were assigned to two groups of individuals, one was assigned to other sites in NZ and AUS (shown in white bar graphs) and another assigned to the same cluster (gray bar graphs). The majority of the individuals were correctly assigned with $80 \%$ or greater probability from their sites. However, the cluster one (ENI) revealed only four (10\%, admixture model) and nine (22.5\%, nonadmixture model) individuals assigned to other groups different than the rest of NZ and AUS individuals. In addition, the best number of clusters was not clear because the $L(K)$ values never reached a plateau after $K=3$, a criterion described in Pritchard et al. (2000). The $\Delta K$ approach described by Evanno et al. (2005) is considered to be a better method for approximating the true $K$, and this approach showed that the best $K$ was two distinct clusters (the highest $\Delta K$ for $K=2$; Figure 4.10), corresponding to a single genetic cluster including all sites in NZ and AUS and another different cluster with CHI. 


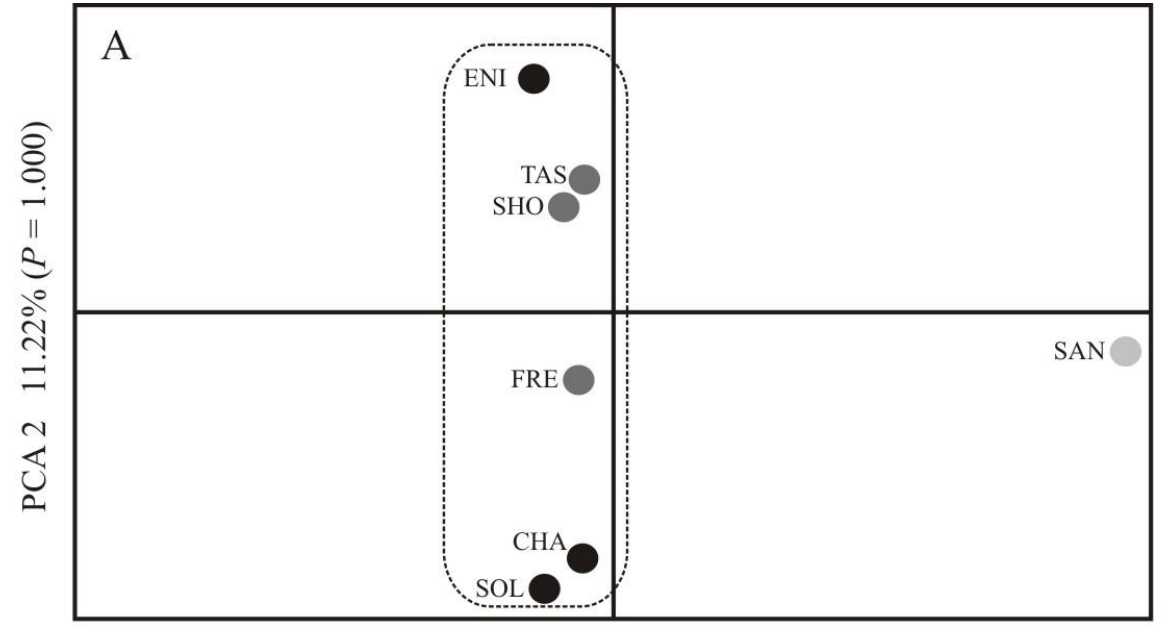

PCA $167.84 \%(P=0.000)$

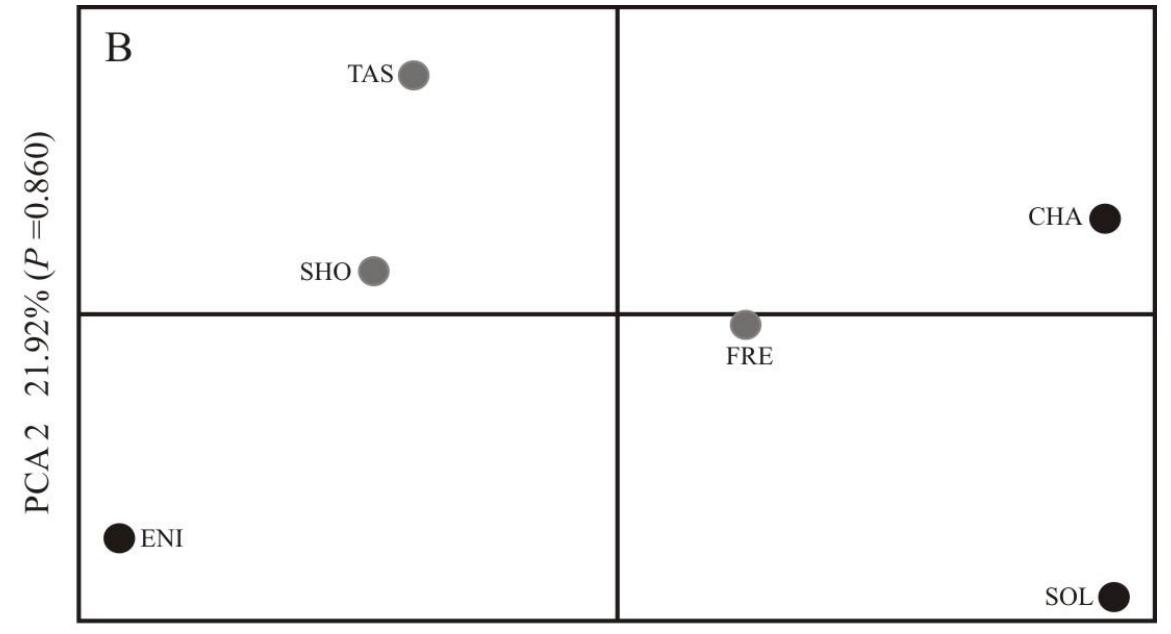

PCA $134.89 \%(P=0.434)$

Figure 4.6: 2D Plot of the first two principal components axes (PCA) of genotype frequencies for eight microsatellite loci (excluding Gg14) from ENI (eastNorth Island), CHA (Chatham Rise), SOL (Solander Island) in New Zealand (black circles); SHO (Shoulder), TAS (West Tasmania), and FRE (Frederich Bay) in Australia (gray circles), and SAN (Santiago) in Chile. Figure A represents all collection sites and B all sites excluding SAN. 


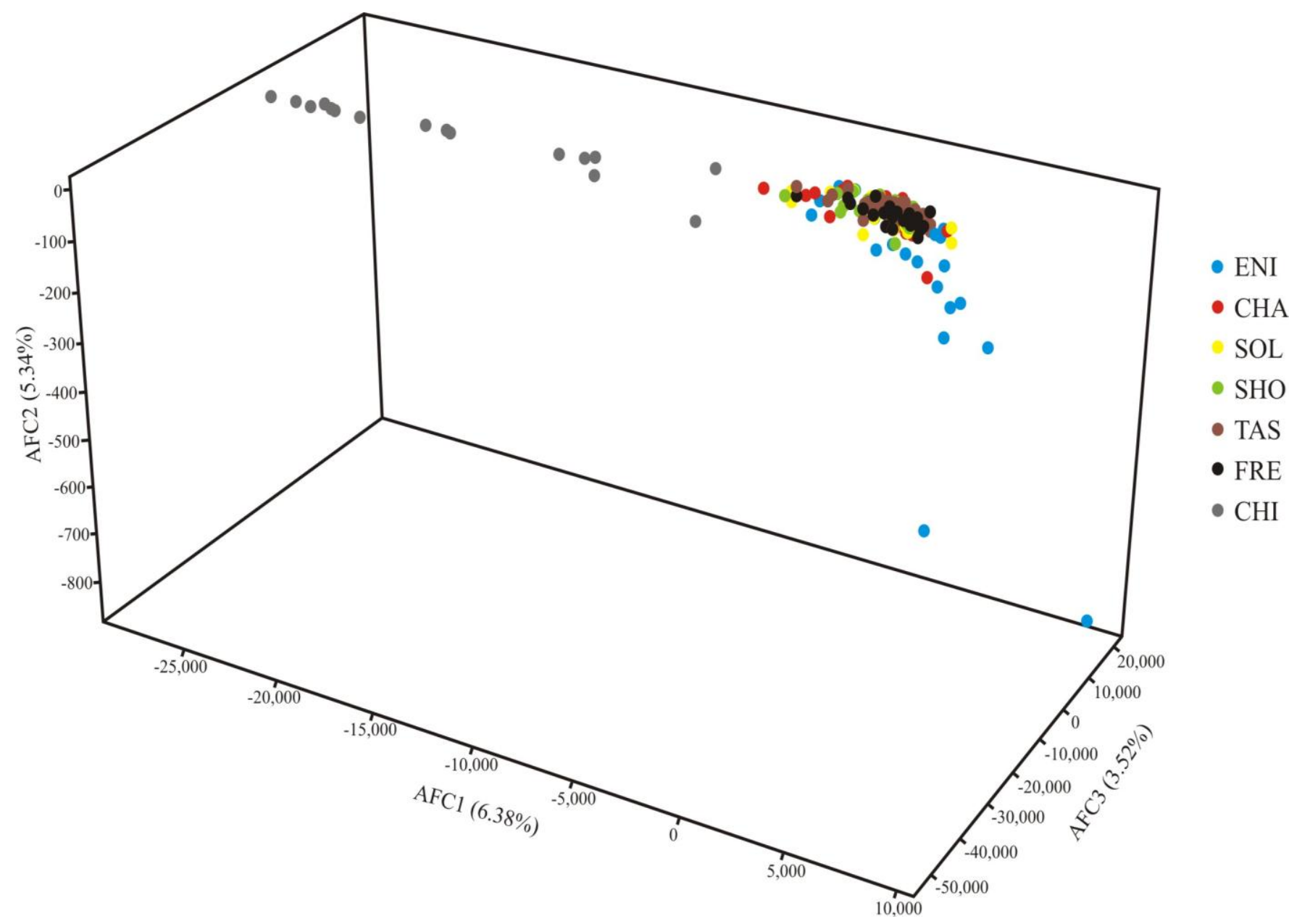

Figure 4.7: 3D Plot of the analyses of principal components (AFC) of genotype frequencies for eight microsatellite loci (excluding $G g 14)$ ). Circles represent each individual and different colours are the populations analyzed: ENI (east-North Island), CHA (Chatham Rise), SOL (Solander Island) in New Zealand; SHO (Shoulder), TAS (West Tasmania), and FRE (Frederich Bay) in Australia; and SAN (Santiago) in Chile. 


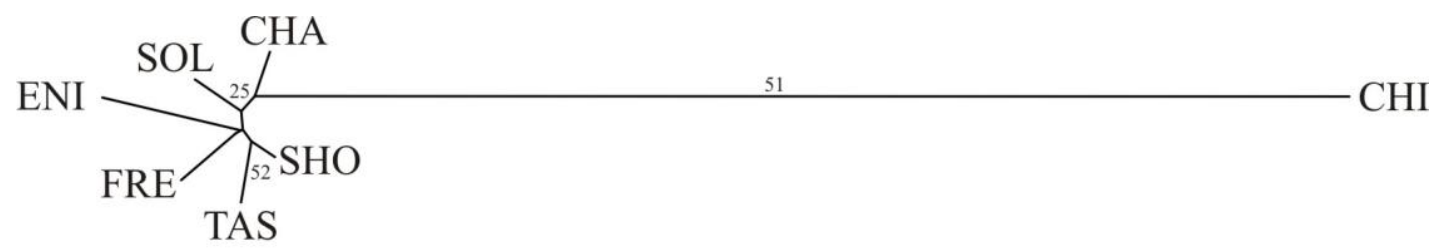

Figure 4.8: Radial neighbor-joining dendrogram for eight microsatellite loci (excluding Gg14) screened from seven populations of G. galeus based on the Nei et al.'s, (1983) Da. Numbers are the bootstrap support indices of loci.

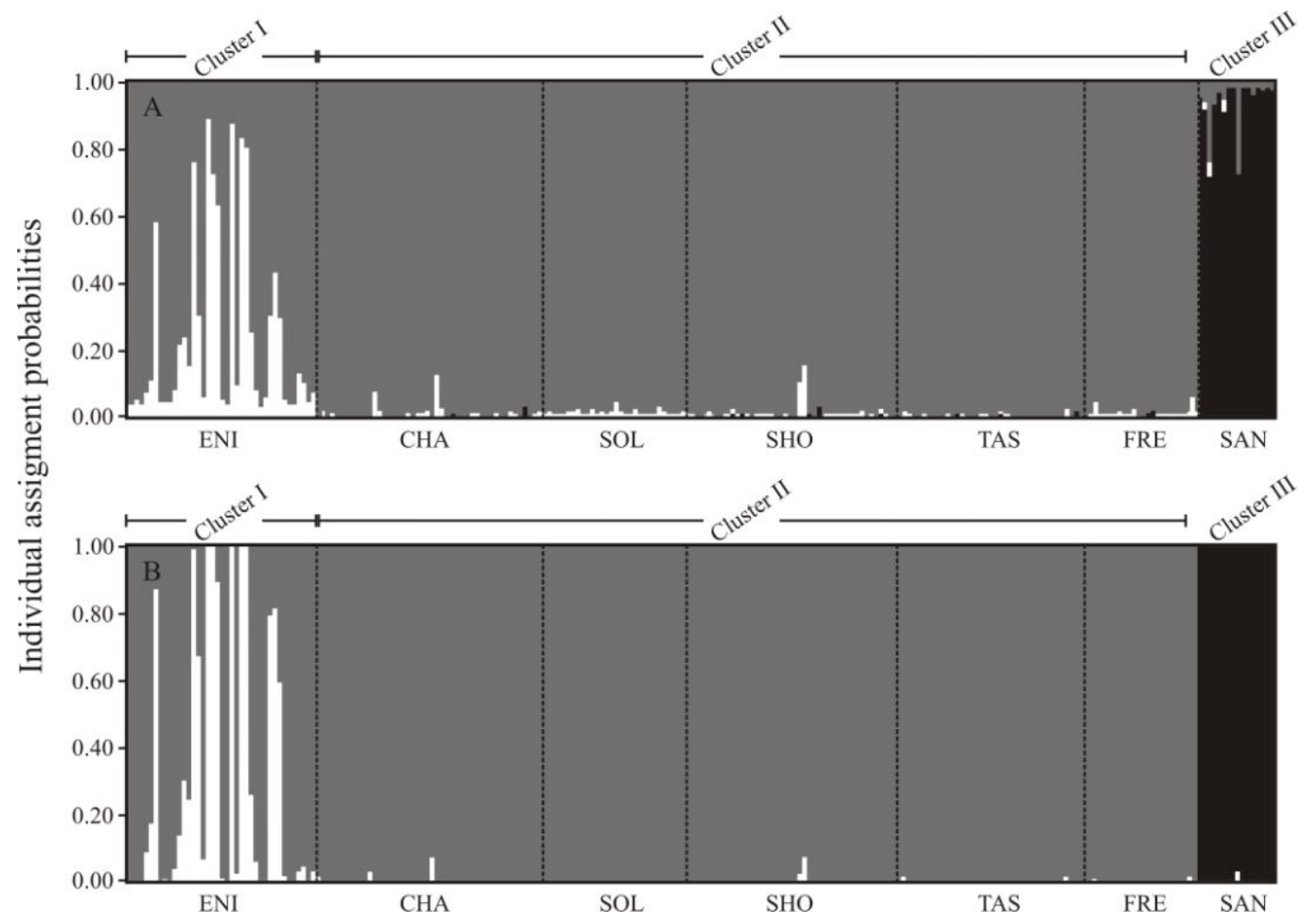

Figure 4.9: Bar plot of Bayesian clustering analysis from G. galeus for eight microsatellite loci (excluding Gg14) (239 individuals) performed using STRUCTURE. Each bar represents proportional probability of assignment to each genetic cluster. Sampling sites were organized by region: New Zealand (ENI, CHA, SOL), Australia (SHO, TAS, FRE), and Chile (SAN). A. Admixture and allele frequencies correlated model for $\mathrm{K}=3$. B. Non-admixture and allele frequencies correlated for $\mathrm{K}=3$.). Black, grey, and white components of each bar represent the proportion in each of the three clusters. 

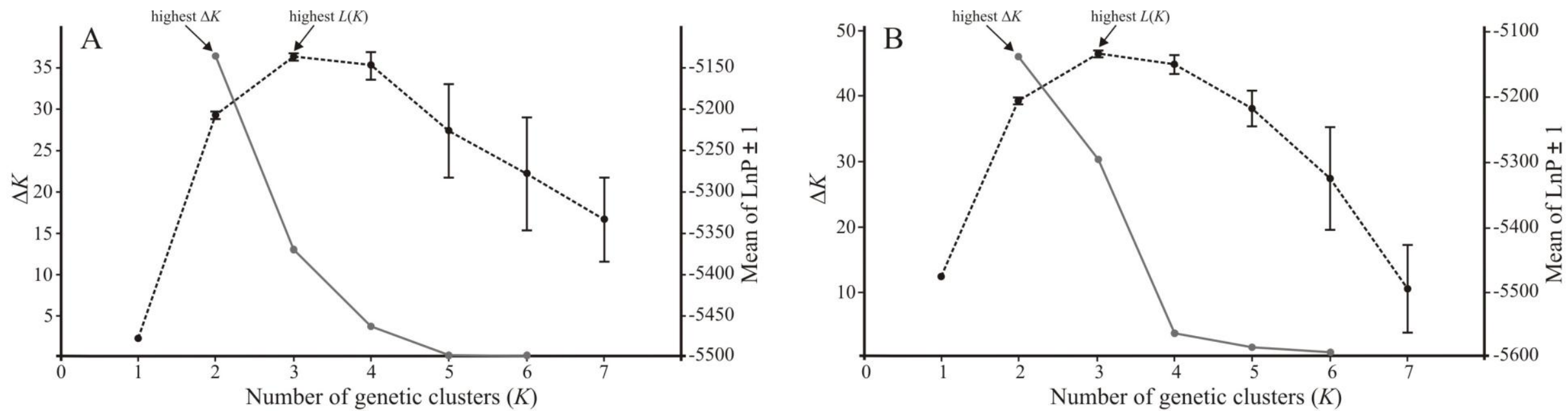

Figure 4.10: Identification of the number of genetic clusters $(K)$ of best fit observed for eight microsatellite loci under "delta $K$ " $(\Delta K)$ and "log probability of data" (Mean of $\mathrm{LnP} \pm 1$ ) approaches. A. Admixture and allele frequencies correlated model for $\mathrm{K}$ ranging from 1-7. B. Non-admixture and allele frequencies correlated for $K$ ranging from1-7. 


\subsubsection{Mutation-drift and migration-drift equilibrium}

The BOTTLENECK analyses did not show any evidence of the genetic effects of a contraction of population size of NZ, AUS and CHI. All sites were in equilibrium under the SMM and TPM mutational models, but the majority of populations were not in mutation-drift equilibrium under the IAM (Table 4.3). The allele frequency distribution showed that the population was in a mutation-drift balance and there was no evidence of genetic population size contraction (Figure 4.11; Table 4.4). In addition, $M$-ratio values for each population were higher than the critical value $(M<$ 0.68) recommended by Garza \& Williamson (2001) (see $M$ values in Table 4.4).

Table 4.4: Results from the BOTTLENECK tests and $M$-RATIO analysis of eight microsatellite loci (excluding $G g 14$ ) for each population. Significant $P$ values for Wilcoxon's test are represented with the asterisks.

\begin{tabular}{|c|c|c|c|c|c|c|c|}
\hline \multirow{3}{*}{ Populations } & \multicolumn{4}{|c|}{ BOTTLENECK } & \multicolumn{3}{|c|}{$M$-RATIO } \\
\hline & \multicolumn{3}{|c|}{ Wilcoxon's test } & \multirow[t]{2}{*}{ Allele frequency distribution } & \multirow[t]{2}{*}{$\theta$} & \multirow[t]{2}{*}{$N_{\text {ef }}$} & \multirow[t]{2}{*}{$M$} \\
\hline & IAM & SMM & TPM & & & & \\
\hline ENI & $0.006^{*}$ & 0.998 & 0.973 & Normal L-shaped & 1.77 & 442.50 & 0.75 \\
\hline $\mathrm{CHA}$ & $0.037^{*}$ & 0.998 & 0.996 & Normal L-shaped & 1.66 & 415.00 & 0.71 \\
\hline SOL & 0.098 & 0.990 & 0.986 & Normal L-shaped & 1.59 & 397.50 & 0.79 \\
\hline $\mathrm{SHO}$ & $0.010^{*}$ & 0.875 & 0.875 & Normal L-shaped & 1.72 & 430.00 & 0.77 \\
\hline TAS & $0.002 *$ & 0.809 & 0.727 & Normal L-shaped & 1.78 & 445.00 & 0.71 \\
\hline FRE & 0.064 & 0.105 & 0.076 & Normal L-shaped & 1.63 & 407.50 & 0.70 \\
\hline SAN & $0.010^{*}$ & 0.875 & 0.875 & Normal L-shaped & 1.53 & 382.50 & 0.77 \\
\hline
\end{tabular}



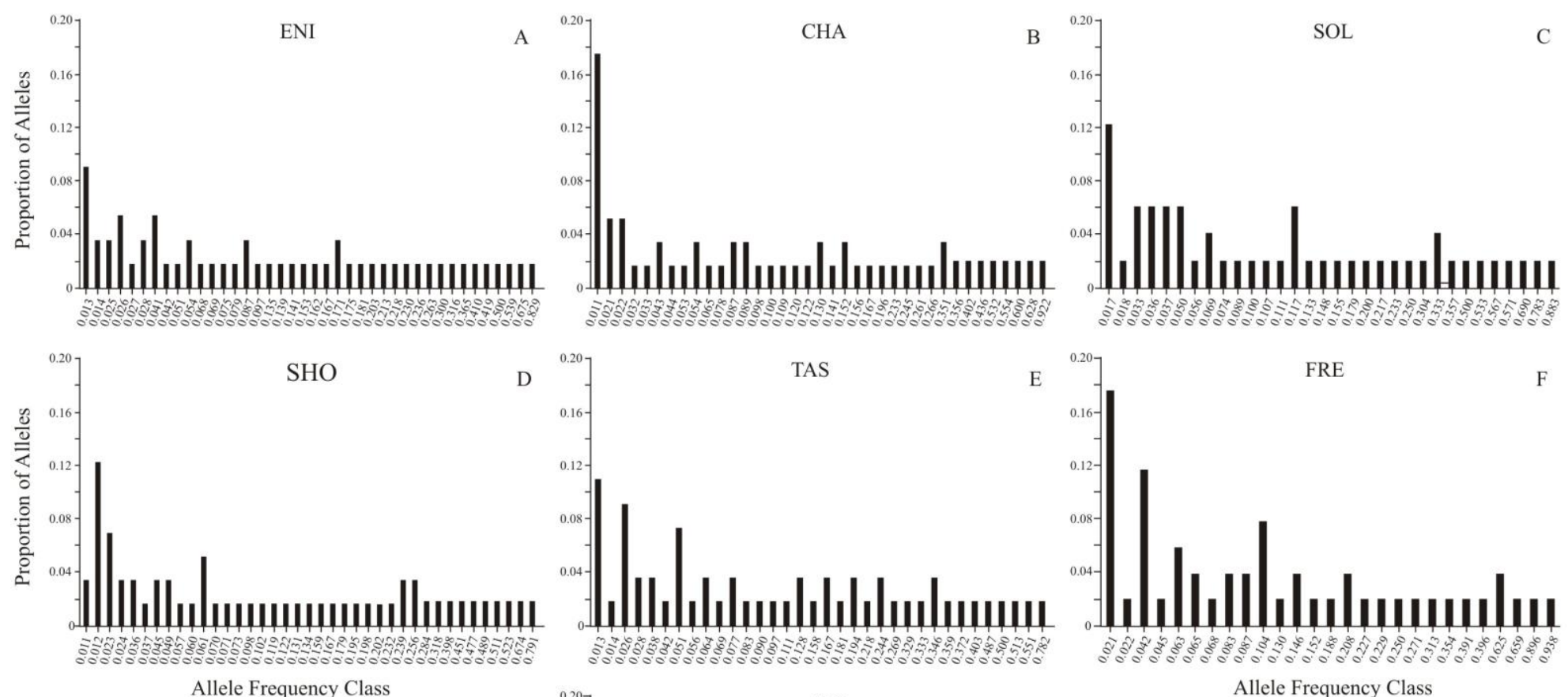

Allele Frequency Clas

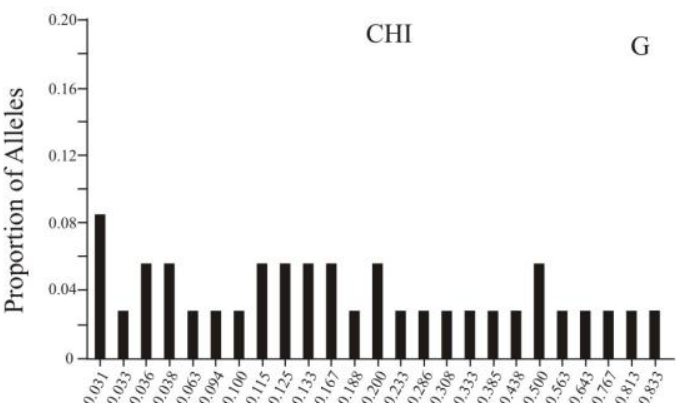

Allele Frequency Class

Allele Frequency Class

Figure 4.11: Distribution of allelic frequencies sizes (X-axis) and the proportion of alleles (Y-axis) for nine microsatellite loci for each population. Black bars represent the proportion of alleles observed in each allele frequency classes. 


\subsection{Discussion}

\subsubsection{Genetic diversity}

Microsatellite DNA markers that have been applied to population genetic studies in elasmobranch species have shown high levels of genetic diversity. A comparison with other species showed that Galeorhinus galeus had a relatively low level of genetic diversity i.e. an average of 5.71 alleles $(A)$, and 0.61 expected heterozygosity averaged $\left(H_{\mathrm{e}}\right)$ across all loci (Table 4.1A in Supplementary material). However, shark species such as Negaprion brevirostris, Carcharhinus leucas, Carcharhinus plumbeus and Isurus oxyrinchus displayed the highest level of genetic diversity in comparison with the rest of elasmobranch species i.e. $A=16.82-28.50$ and $H_{\mathrm{e}}=0.79-0.87$ (Schrey \& Heist 2003, Feldheim et al. 2001, Schultz et al. 2008, Portnoy et al. 2010, Karl et al. 2011). The findings of these studies were based on highly polymorphic microsatellite loci. The higher number of repeat motifs (>20 repeats) and perfect (uninterrupted) motif sequences have produced a data set with more distinguishable alleles and consequently increased the level of genetic resolution.

Genetic diversity indexes are generally low in elasmobranch species $(A=10.39$ and $\left.H_{\mathrm{e}}=0.63\right)$ in comparison with other marine fishes such as Actinopterygii i.e. ray-finned species that have higher number of alleles per locus $(A=19.90)$ and higher heterozygosities $\left(H_{\mathrm{e}}=0.77\right)$. However, genetic diversity in elasmobranchs is relatively similar to Actinopterygii freshwater and anadromous fish species (Table 4.1B and C in Supplementary material). The differences in levels of genetic diversity between groups of fishes might reflect differences in their average effective population sizes. Most Actinopterygii marine species have external fertilization with high fecundity, producing thousands of viable eggs which lead to large and continuous populations. Subsequently, free-swimming adults are also good dispersers and contribute to genetic connectivity among subpopulations. In comparison with Actinopterygii marine species, elasmobranchs show lower potential for dispersal during their early life stages. For example, species such as G. galeus do not migrate long-distance in open water after 2+ years (Olsen 1954, Hurst et al. 1999, Francis 2010). In addition, the low genetic diversity of elasmobranchs may reflect their $K$-selected strategies such as extremely low fecundity (15-52 pups for G. galeus), long reproductive cycles (triennial for G. galeus), and late sexual maturity (8-17+ years for G. galeus) (Olsen 1954, 1984, Peres \& Vooren 1991, Francis \& Mulligan 1998, Walker 2005). These characteristics give elasmobranch species low reproductive potential in comparison with Actinopterygii marine species. 
Although the levels of genetic diversity were low in G. galeus when compared to other elasmobranch species, the allelic richness $\left(R_{s}\right)$ and expected heterozygosity $\left(H_{\mathrm{e}}\right)$ were different among populations. There were different levels of genetic diversity in New Zealand/Australia compared with Chile for several loci i.e. Ggal14, Gg20, Gg12, Gg7, and Gg11. This finding was consistent with the patterns of allele distributions, for example, 11 alleles were not present for loci $G g 12$ in Chile when compared to the New Zealand/Australian sample. The absence of alleles in one of the populations indicates a longstanding barrier to gene flow and independent demographic histories in populations on each side of the South Pacific Ocean (SPO). This pattern is consistent with the results of the mtDNA CR study presented in Chapter 2, which revealed that the level of genetic diversity (i.e. haplotype and nucleotide diversity) were higher in Chile than New Zealand/Australia.

\subsubsection{Genetic differentiation and population structure}

Marine populations of elasmobranch species are thought to have high levels of genetic connectivity because of their high capacity for dispersal and the lack of biogeographic barriers or distance between habitats. However, life history traits and behaviour biology of some species can also limit gene flow. Elasmobranch species often have low reproductive potential, and also differential patterns of female and male migration. Females of some species show site fidelity or philopatry returning to a home area, birthplace or another locality during part of their life cycles, whereas males roam and disperse across their whole geographic distribution (Hueter et al. 2005). This observation has been supported by tagging/tracking migration studies and contrasting the degree of significant population structure between mtDNA (maternally inherited) and lack of structuring in microsatellites (biparentally inherited). Sex-biased philopatry has been reported for Negaprion brevirostris (Feldheim et al. 2002), Carcharhinus limbatus (Keeney et al. 2005), Carcharodon carcharias (Pardini et al. 2001, Blower et al. 2012), Carcharhinus plumbeus (Portnoy et al. 2010), and Carcharhinus leucas (Karl et al. 2011, Tillet et al. In review). The level of gene flow is typically determined by different behavioural and biological characteristics of species, which can affect the strength of genetic drift and natural selection (Palumbi 1994, Graves 1998, Dudgeon et al. 2012).

In contrast to the results of the mtDNA markers in elasmobranch species, microsatellite loci have shown evidence of genetic differentiation within ocean basins. High 
level of population structure are found in highly vagile shark species have been found in samples from Atlantic and Pacific Oceans in Isurus oxyrinchus (Schrey \& Heist 2003), Pacific and Atlantic in Cetorhinus maximus (Hoelzel et al. 2006), Indo-Pacific Ocean in Negaprion acutidens (Schultz et al. 2008), Pacific and Indian Oceans in Rhincodon typus (Schmidt et al. 2009), and across SPO for Squalus acanthias (Veríssimo et al. 2010). Conversely, higher levels of genetic connectivity were found for less vagile shark species that were coastal and benthonic with more restricted geographic distribution. For example, Stegostoma fasciatum exhibited population genetic differences between northern Australia and southeast Asia (Dudgeon et al. 2008), Carcharhinus leucas between northern coast of Brazil and gulf coast of Florida (Karl et al. 2011), Carcharias taurus between Brazil and South Africa (Ahonen et al. 2009), and Raja clavata between the Mediterranean basin, the Azores and the European continental shelf (Chevolot et al. 2006). At a small spatial scale, microsatellite markers have demonstrated that juveniles of Carcharodon carcharias are philopatric suggesting the presence of discrete nursery areas on the eastern and southwestern Australian coastlines (Blower et al. 2012). The genetic studies have provided a better understanding of the connectivity of elasmobranch species and their dispersal patterns and the traditional view about barriers (biological and/or biogeographic) to gene flow have been revised in this Chapter and Chapter 2.

At a large spatial scale, the results revealed a lack of genetic connectivity of G. galeus across SPO between populations of New Zealand/Australia and Chile. However, there was a high level of gene flow across the Tasman Sea between New Zealand and Australian populations. The sampling of G. galeus conducted for this study provided a better coverage of the North Island and South Island in New Zealand and along the Southern Shark Fishery in Australia i.e. including South Australian, Victorian and Tasmanian waters (McLoughlin 2007). The results indicated that G. galeus in New Zealand and Australia represent a single panmictic population. This finding was consistent with the results from the mtDNA CR that were reported in Chapter 2. Both the nuclear and mtDNA markers suggested that there is a substantial level of gene flow among sites in New Zealand and Australian waters, which is a conclusion also supported by extensive tagging data that indicate high migration rate across Tasman Sea (Hurst et al. 1999, Walker et al. 2008, Francis 2010). The similar patterns found for these markers, which have difference patterns of inheritance (i.e. nuclear is biparentally inherited and mtDNA is maternally inherited) suggest than there is no evidence of sex-biased dispersal. Female G. galeus are not expected to be philopatric to a specific reproductive 
territory. However, it may also be possible that the mtDNA does not have enough resolution power to detect a pattern of philopatry in G. galeus.

\subsubsection{Mutation-drift equilibrium}

A reduction in population size can lead to the loss of genetic variation. It also can increase rates of inbreeding, the fixation of deleterious alleles, and reduce the adaptive potential of populations (Frankham et al. 2002, Allendorf \& Luikart 2007). Population genetic theory predicts that rare alleles will be more readily affected by drift than more frequent alleles (Nei et al. 1975, Hedrick 2000). The presence of rare alleles in the New Zealand, Australia and Chile G. galeus populations suggest that the possible population size contraction caused by exploitation has had no appreciable effect on the pattern of genetic variation. This finding could be because not enough time has elapsed, but after a few more generations the loss of rare alleles could be a detectable pattern. The finding was also consistent with results of the mtDNA reported in Chapter 2. It is possible that the Australasian G. galeus populations are at mutational-drift equilibrium, even though they have experienced high levels of exploitation.

\subsubsection{Management implication}

It is important to note that population genetic findings reflect the long-term evolutionary processes of a species and their populations. In other words, genetic markers detect patterns of gene flow that have become established over thousands of years. Consequently, they are not often able to detect more recent changes in population size and migration/recruitment patterns typically caused by overfishing, habitat degradation or other anthropogenic disturbance. Nevertheless, if populations were declining then contemporary levels of migration might also be in decline. Species such as G. galeus are highly susceptible to overfishing and habitat degradation due to their low reproductive potential (e.g. low fecundity). Even when molecular markers have revealed historical migration patterns of species, it is important to investigate the extent of the movement and interbreeding between populations. This information is fundamental to identifying the number of populations, and the genetic connectivity among populations of vulnerable and commercially fished elasmobranch species. 
This study reports for the first time the patterns of population genetic variation for $G$. galeus in New Zealand, Australia and Chile based on microsatellite loci. There was evidence of genetic differentiation across SPO between New Zealand/Australia and Chile, but no evidence of differentiation between New Zealand and Australia. The Trans-Tasman connectivity was consistent with previous tagging studies that suggested high migration rates across the Tasman Sea (Hurst et al. 1999, Walker et al. 2008, Francis 2010). The genetic data demonstrate that the migrants were reproductively successful and there were enough of them to genetically homogenise the Australasian stock (Waples 1998). Galeorhinus galeus is a fished species that is able to cross geographic and international management boundaries. This finding supports the suggestion that there should be a co-ordinated Australian and New Zealand management perspective on fishery. However administrative and logistic considerations in New Zealand and Australia have meant that this species is assessed and managed as separate stocks in each country, even though studies have now shown that they are most likely a single panmictic stock. It is probably time to re-examine the management of G. galeus and consider a single-stock management approach. The current TAC (total allowable catch) levels in New Zealand waters should be re-evaluated because the stock is considered "sustainable" (MPI 2012) in New Zealand but "overfished" in Australian.

In conclusion, this study demonstrated that G. galeus populations are genetically discrete population across SPO i.e. South Hemisphere (New Zealand, Australia and Chile). The use of CR of mtDNA (see Chapter 2) and microsatellite loci (the present Chapter) in three locations are fairly consistent with the thought that G. galeus is composed of a single panmictic stock in New Zealand and Australia. However, further genetic studies may provide a more comprehensive understanding of the patterns of genetic connectivity of G. galeus in the Australasia region, and investigate the possibility of temporal variation using samples of neonates, pups, juveniles and females from widely separated nursery areas. This approach would provide valuable and better information about the degree of site fidelity or philopatry in G. galeus. 


\subsection{Appendices}

\section{Supplementary Material}

Supplementary Table 4.1: A. Genetic diversity at microsatellite loci reported to date for population genetic studies among 16 Elasmobranch species. Number of populations studied ( $N$ pop), number of loci ( $N$ loci), number of individuls screened across loci $(N$ ind), number of alleles averaged across all loci $(A)$, and expected heterozygosity averaged across all loci $\left(H_{\mathrm{e}}\right)$ are reported. Note for Carcharodon carcharias only observed heterozygosity $\left(H_{\mathrm{o}}\right)$ was reported in Pardini et al. (2001). B. Genetic diversity at microsatellite loci in teleost freshwater, teleost anadromous, teleost marine, and elasmobranch species. C. Graphical representation of Table B for four different groups of species.

\begin{tabular}{|c|c|c|c|c|c|c|c|c|c|}
\hline Species & \multicolumn{3}{|c|}{$N$ pop } & $N$ loci & $N$ ind & \multicolumn{2}{|l|}{$A$} & $H_{e}$ & References \\
\hline \\
\hline \multicolumn{2}{|l|}{ Carcharhinus plumbeus } & \multicolumn{2}{|c|}{2} & \multicolumn{2}{|r|}{71} & \multicolumn{2}{|c|}{$2.33(0.47)$} & $0.28(0.19)$ & Heist \& Gold 1999 \\
\hline \multicolumn{2}{|l|}{ C. plumbeus } & \multicolumn{2}{|c|}{9} & 8 & $333-336$ & \multicolumn{2}{|c|}{$16.82(10.31)$} & $0.83(0.13)$ & Portnoy et. al. 2010 \\
\hline \multicolumn{2}{|l|}{ C. limbatus } & \multicolumn{2}{|c|}{9} & 8 & 419 & \multicolumn{2}{|c|}{$8.67(8.53)$} & $0.51(0.33)$ & Keeney et. al. 2005 \\
\hline \multicolumn{2}{|l|}{ C. sorrah } & \multicolumn{2}{|c|}{4} & 5 & $140-204$ & \multicolumn{2}{|c|}{$10.80(8.54)$} & $0.54(0.32)$ & Ovenden et. al. 2009 \\
\hline \multicolumn{2}{|l|}{ C. obscurus } & 3 & & 4 & $48-98$ & $7.92(5.5$ & & $0.73(0.14)$ & Ovenden et. al. 2009 \\
\hline C. leucas & & 4 & & 5 & $124-145$ & $19.15(9$ & & $0.85(0.17)$ & Kark et. al. 2011 \\
\hline Negaprion brevirostris & & 3 & & 4 & 2632 & $28.50(9$ & & $0.79(0.08)$ & Feldheim et. al. 2001 \\
\hline$N$. brevirostris & & 4 & & 9 & 80 & $19.80(\mathrm{~N}$ & & $0.81(\mathrm{NR})$ & Schultz et. al. 2008 \\
\hline N. acutidens & & 5 & & 6 & 68 & $10.50(\mathrm{~N}$ & & $0.67(\mathrm{NR})$ & Schultz et. al. 2008 \\
\hline Sphyrna lewini & & 3 & & 3 & $149-168$ & $6.44(4.3$ & & $0.54(0.35)$ & Ovenden et. al. 2009 \\
\hline Ginglymostoma cirratum & & 3 & & 3 & $145-151$ & $5.83(4.3$ & & $0.58(0.20)$ & Kark et. al. 2012 \\
\hline Prionace glauca & & 4 & & 5 & $64-118$ & $6.60(4.4$ & & $0.57(0.20)$ & Ovenden et. al. 2009 \\
\hline Isurus oxyrinchus & & 8 & & 4 & $421-430$ & $25.25(12$ & $.32)$ & $0.87(0.05)$ & Schrey \& Heist 2003 \\
\hline Carcharodon carcharias & & 2 & & 5 & 95 & $7.00(3.6$ & & $0.48(0.19)$ & Pardini et. al. 2001 \\
\hline Carcharias taurus & & 6 & & 6 & 186 & $5.03(3.1$ & & $0.54(0.28)$ & Ahonen et. al. 2009 \\
\hline Squalus acanthias & & 9 & & 8 & 419 & $7.11(3.1$ & & $0.61(0.04)$ & Veríssimo et. al. 2011 \\
\hline Rhincodon typus & & 10 & & 8 & 68 & $9.00(9.5$ & & $0.69(0.20)$ & Schmidt et. al. 2009 \\
\hline Galeorhinus galeus & & 7 & & 9 & $228-235$ & $5.71(2.7$ & & $0.61(0.19)$ & This study \\
\hline$\frac{\text { Batoidea }}{\text { Raja clavata }}$ & & 11 & & 5 & 214 & $9.72(7.0$ & & $0.72(0.19)$ & Chevolot et. al. 2008 \\
\hline$\underline{B}$ & & & & & & & & & \\
\hline Group of species & $N$ loc & & $N \mathrm{sp}$ & & $A$ & & $H_{e}$ & $\mathrm{Re}$ & erences \\
\hline$\frac{\text { Actinopterygii }}{\text { Teleost freshwater }}$ & 75 & & 13 & & & $(6.10)$ & 0.54 & $(0.25)$ & Noody \& Avise 2000 \\
\hline Teleost anadromous & 43 & & 7 & & & $30(7.20)$ & 0.68 & $(0.12)$ & Noody \& Avise 2000 \\
\hline Teleost marine & 66 & & 12 & & & $0(6.60)$ & 0.77 & $(0.19)$ & Noody \& Avise 2000 \\
\hline $\begin{array}{l}\text { Chondrichthyes } \\
\text { Elasmobranches }\end{array}$ & 86 & & & & & & & & \\
\hline
\end{tabular}

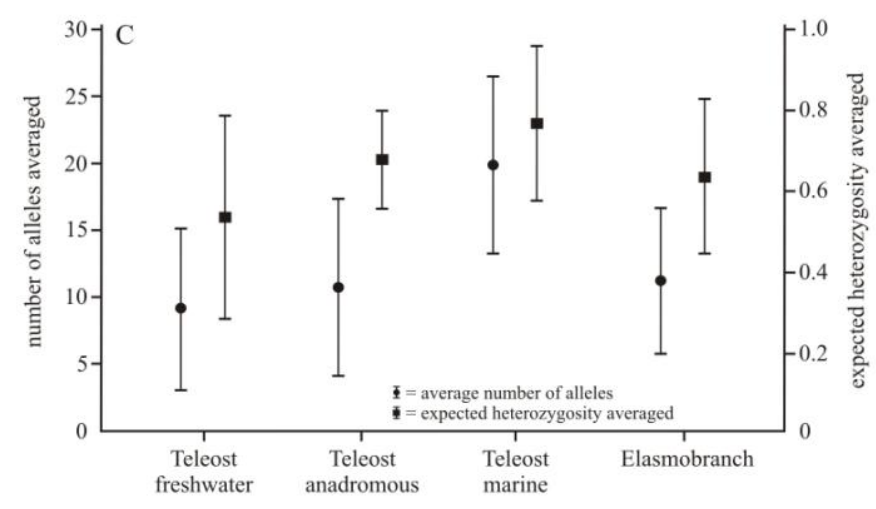




\section{Chapter 5}

\section{Paternity assessment in school shark Galeorhinus galeus in New Zealand waters}

\subsection{Abstract}

Sharks can be either monogamous or polygamous, which is important when considering stock assessments and harvesting models. Multiple paternity has been reported in several shark species, however, the number of sires per litter varies considerably among species. The aim of this study was to determine whether there was multiple paternity (MP) in G. galeus by assessing the levels of relatedness of progeny arrays using six polymorphic microsatellite DNA markers. Five "families" (mother and litters) were sampled from the North Island of New Zealand and a parentage analysis was conducted. The minimum number of males contributing to each progeny array was estimated by identifying the putative paternal alleles by manual allele counting and reconstructing multilocus genotypes method. The analysis showed the occurrence of genetic polyandry in G. galeus. Two of five litters showing multiple sires involved in the progeny arrays (40\%). The minimum number of sires per litter ranged from one to four. Although MP was only detected in a two litters, this finding is consistent with the known reproductive characteristics of G. galeus. Females can potentially store sperm for long periods of time and the species has a specific mating season when males and females typically mix on the edge of continental shelf. Detecting MP within a litter has highlighted the importance of the post-copulatory selective processes in the G. galeus mating system, and this has implications for fishery management and conservation. 


\subsection{Introduction}

Elasmobranch species have a range of reproductive modes such as oviparity, aplacental viviparity and placental viviparity (Wourms 1977, Wourms \& Demski 1993, Grogan \& Lund 2004). Within this group, species showed difference in ovarian cycles, gestation periods, and mating systems, but generally they exhibit a low reproductive potential e.g. slow growth rate, late age of sexual maturation, low fecundity. The $K$-selected features make elasmobranch vulnerable to fishing exploitation, caused by a reduction in reproductive capacity and effective population size (Smith et al. 1998, Stevens et al. 2000, Myers \& Worm 2003, Dichmont et al. 2011). Fisheries managers and scientists have developed strategies to ensure the sustainable use of biological resource and maintain the productivity of a stock (Musick \& Bonfil 2005). Crucial to management is a robust stock assessment (demographic and behaviour), which should include information about reproductive cycles and mating system (Rowe \& Hutchings 2003).

Sharks litters can be either monandrous or polyandrous. Depending of the mating system, species can be affected in term of genetic variability and interbreeding, even varying the ability for adaptation within population (Sugg \& Cheseer 1994, Frankham 2005). For instance, species that the number of males contributing to the gene pool is small, they lead to have very small effective population sizes and consequently make them vulnerable to overfishing. It is known that both mating strategies (i.e. monogamy and polyandry) have several benefit and cost. Thus, mating with multiple males may expose females to higher risks from sexually transmitted diseases, predation, copulation brutality, or other time or energy expenses associated with the mating process (Avise et al. 2002, Byrne \& Avise 2012, Fitzpatrick et al. 2012). However, these risks could be outweighed by the benefits increased female fitness, for example as insurance against male sterility, offspring with more diverse genotypes, and inbreeding avoidance (Avise et al. 2002, Frankham 2005) These theoretical considerations suggest that promiscuity in elasmobranch can be genetically advantageous for females and males.

Molecular markers have been used to study mating systems and sexual selection. Fishes species have showed a diverse range of breeding behaviors and they have been a useful group for testing theories regarding mating systems and reproductive strategies using genetic markers e.g. microsatellite loci, in conjunction with behavior observations (Avise et al. 2002, Fitzpatrick et al. 2012). These finding have demonstrated that it is common for 
females to copulate with multiple males (polyandry) and offspring are a mixture of full and half-sibs (Zeh \& Zeh 2003). The evidence of polyandry in females of shark species has increased, and multiple mating is common reproductive strategy (see review in Byrne \& Avise 2012, Fitzpatrick et al. 2012). Mating systems in elasmobranchs has been distinguished using: a) Direct behavioral observation of multiple mating in field described for Ginglymostoma cirratum (Carrier et al. 1994, Pratt \& Carrier 2001), Triaenodon obesus (Whitney et al. 2004), Manta birostris (Yano et al. 1999), and Dasyatis americana (Chapman et al. 2003); and observation of captive sharks e.g. Scyliorhinus rotifer (Castro et al. 1988) and Carcharias taurus (Gordon 1993); and b) Genetic studies that assessed multiple paternity (MP) of offspring i.e. microsatellites loci are commonly used in several species such as: Negaprion brevirostris (Feldheim et al. 2001a, 2004, DiBattista et al. 2008), Ginglymostoma cirratum (Saville et al. 2002, Heist et al. 2011), Sphyrna tiburo (Chapman et al. 2004), Carcharhinus altimus and C. galapagensis (Daly-Engel et al. 2006), Carcharhinus plumbeus (Daly-Engel et al. 2006, 2007, Portnoy et al. 2007), Squalus acanthias (Lage et al. 2008, , Veríssimo et al. 2011), Squalus mitsukurii (Daly-Engel et al. 2010), Rhincodon typus (Schmidt et al. 2010), Mustelus henlei (Byrne \& Avise 2012), and Scyliorhinus canicula (Griffiths et al. 2012), and one skate species, Raja clavata (Chevolot et al. 2007).

Microsatellite markers are used for genetic parentage analyses because they have a Mendelian pattern of inheritance (Avise 2002, Chapman et al. 2004). Basic analytical methods and sophisticated statistical approaches have been used for parentage assessment. Subtracting the more easily identified maternal alleles allows the paternal alleles to be identified, because the mother can usually be sampled at the same time as the offspring. Identifying the parental alleles in progeny has been used to determine the number of sires within the progeny array of a mother. Based on this notion, several reconstructions multilocus genotypes methods have been developed (e.g. GERUD software), which simulate increase the knowledge about mating system in several taxa such as birds, mammals, reptiles and elasmobranch species.

The school shark (Galeorhinus galeus) is a mobile and semipelagic/demersal shark species found globally in temperate waters of the eastern North Atlantic, western South Atlantic, north eastern and south eastern Pacific, South Africa, New Zealand and Southern Australia (Last \& Stevens 2009). Commercially fished Australasia region where it is considered different stock across Tasman Sea (between New Zealand and Australia) for management purposes, but according to the results described in Chapter 2 (mtDNA) and 4 
(microsatellite loci), G. galeus populations are composed of a single stock unit. Several stocks of G. galeus are in significant decline, prompting the species to be listed as vulnerable by the IUCN Red List of Threatened Species (Walker et al. 2006). In the Southwest Atlantic the G. galeus population is subject to intensive fishing such as: a) in Brazil and Uruguay, the CPUE has declined almost to zero; b) In Argentina, the CPUE for the trawler fleet has declined by around $80 \%$ during the past decade (Walker et al. 2006). In Australia, G. galeus fisheries have reduced the stock to less than $15 \%$ of initial biomass, raising serious concerns (AFMA 2009, 2010a, DSEWPaC 2012). The TAC dropped (total allowable catch) from $800 \mathrm{t}$ in 2002 to $240 \mathrm{t}$ in 2010 (AFMA 2010b). Proper management is critical because the low reproductive potential of $G$. galeus makes it particularly vulnerable to overexploitation.

Galeorhinus galeus, have internal fertilization and aplacental viviparity. Fecundity varies between 15-52 pups depending on the size and age of the mother (Olsen 1954, 1984, Walker 2005). Sexual maturity occurs later in females compared to males. Females reach maturity at about $13-15+$ years (124-140 cm total length, TL), whereas in males it is at about 8-17+ years (125-135 cm TL) (Olsen 1954, 1984, Francis \& Mulligan 1998, Walker 2005). The reproductive cycle in females is characterised by a long ovarian and gestation period estimated to be three years (triennial) (Olsen 1984, Peres \& Vooren 1991, Walker 2005). Females give birth approximately every 3 years (Peres \& Vooren 1991, Walker 2005). This means that G. galeus population will comprise females at three different reproductive stages: a) individuals with resting oviducts (after parturition) and vitellogenesis in the ovary, b) individuals with large mature follicles, oviducal gland and uteri ready for ovulation, and c) others with full-term embryos in the oviducts (Peres \& Vooren 1991, Walker 2005). These portions mean that on average one-third of the mature females in a population will be producing the annual recruitment of newborns.

An understanding of G. galeus mating system is a fundamental requirement for any long-term, effective fishery management because the level of shark exploitation worldwide exceeds their reproductive capacity (Manire \& Gruber 1990, Stevens et al. 2000, Baum et al. 2003, Myers \& Worm 2003). Mating system information is important because reproductive strategies, such as polyandry, could make the population demographics more sensitive to fishing-induced mortality (Sugg \& Cheseer 1994, Martinez et al. 2000, Chapman et al. 2004). 
The goal of this chapter was to determine the pattern of parentage of five G. galeus families (mums and embryos) sample from North Island of New Zealand. The family groups were screened using six highly polymorphic microsatellite loci (see details in Chapter 4). 


\subsection{Methods}

\subsubsection{Sampling collection}

Five G. galeus mother-litters groups (hereafter called a Family) were collected from two breeding grounds (Kaipara and Manukau harbours) located in the North Island of New Zealand. Four gravid females were captured using rod lines from Kaipara harbour and one female from Manukau harbour over two summer seasons in 2009 and 2012 (see details in Figure 5.1). Fins clips where taken from the mother and whole embryos were frozen and then transferred to the laboratory. The total length (TL) of each individual was recorded. In order to obtain reasonable sample sizes for the population genetic data of G. galeus (i.e. allele diversity), the tissues samples that were reported in Chapters II and IV from east of North Island (ENI, $n=40$ ) were pooled with the genotyping data obtained from five pregnant females (KP, $n=4$; MK, $n=1)$.

Figure 5.1: A. Collection location of G. galeus mother-litters groups (solid circles) of two putative breeding grounds on Kaipara (KP) and Manukau (MK) harbours, and east of North Island (ENI) samples used for population genetic analysis in this study. Numbers in brackets are the sample sizes. B. Table represents G. galeus gravid females (named as Mum) analysed in this study. Total length (TL) is showed in $\mathrm{cm}$ and LS represents litter size.

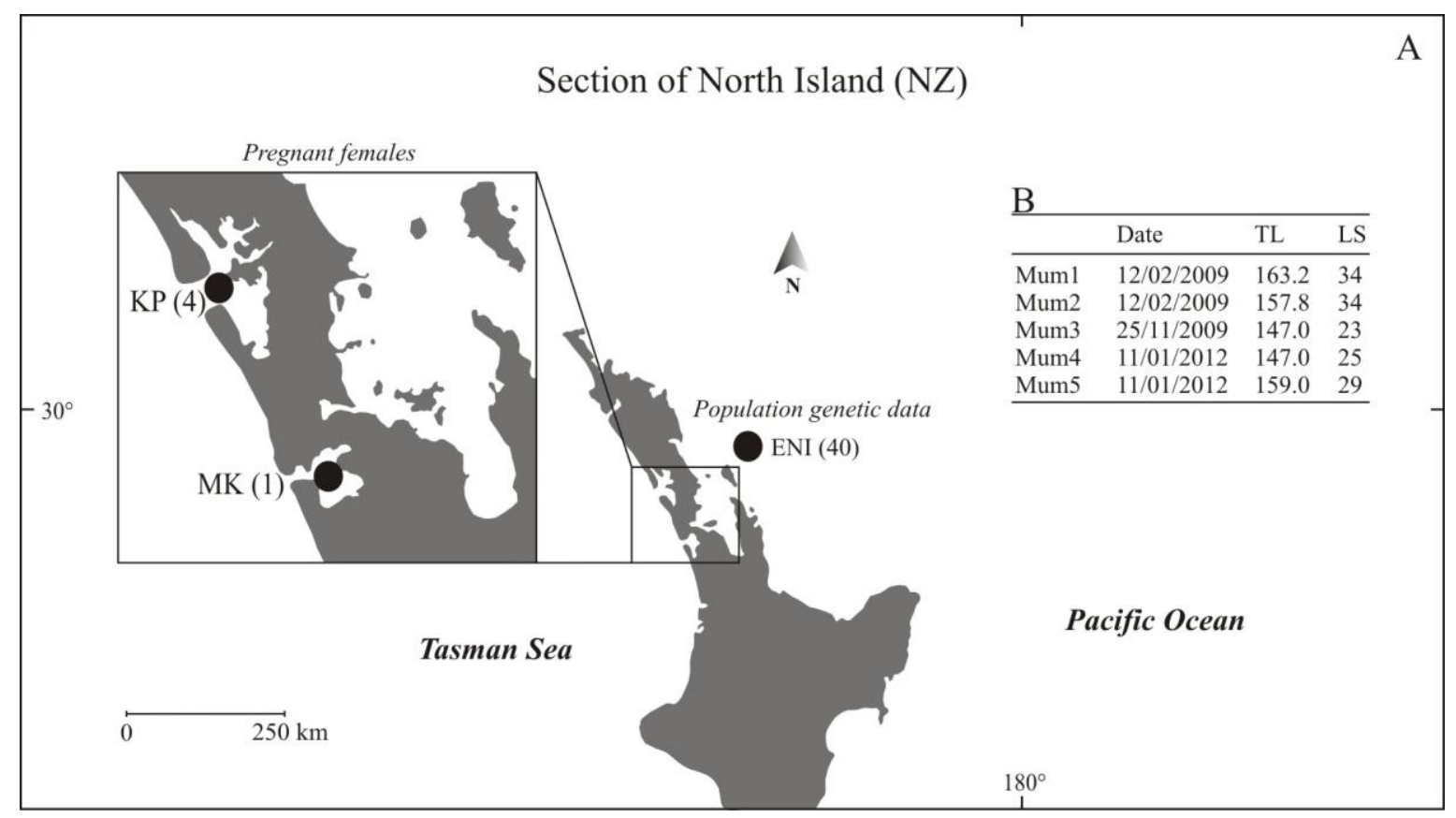




\subsubsection{DNA Extraction and Genotyping}

Genomic DNA was extracted from tissue samples using a standard phenol-chloroform protocol (Sambrook et al. 1989). Extracted DNA was checked for quality and quantified using a NanoDrop spectrophotometer (model ND-1000), and all samples were diluted to a standardised concentration of $50 \mathrm{ng} / \mu \mathrm{L}$. All the individuals were genotyped using six microsatellite loci $G g 15, G g 22, G g 23, G g 12, G g 7$, and $G g 11$. All loci ( $G g 22, G g 23, G g 12$, $G g 7$, and $G g 11$ ) were taken from Chabot \& Nigenda (2011). These loci had a high level of allelic diversity, which is needed to detect MP in the pup arrays. All details about the microsatellite loci (i.e. primer sequences and PCR conditions) have been presented in Chapter 3 and 4. Microsatellite loci were PCR amplified separately in $10 \mu 1$ volumes containing 67 $\mathrm{mM}$ Tris-HCL pH 8.8, $16 \mathrm{mM}\left(\mathrm{NH}_{4}\right)_{2} \mathrm{SO}_{4}, 2.0 \mathrm{mM} \mathrm{MgCl}, 0.05 \mu \mathrm{M}$ of forward primer labelled with M-13 tail, $0.2 \mu \mathrm{M}$ of reverse primer, $0.05 \mu \mathrm{M}$ of fluorescently labelled $\mathrm{M}-13$ primers (FAM, VIC, or PET Invitrogen), $0.8 \mathrm{mM}$ of each dNTPs, 0.3 units of BIOTAQ ${ }^{\mathrm{TM}}$ DNA Polymerase (Bioline), and $1 \mu \mathrm{l}$ of template DNA (50-80 ng/ $\mu 1$ DNA concentration). Thermal cycling was performed on a TGradient Thermal Block (Biometra, Goettingen, Germany). Primer pairs and PCR conditions for each locus can be found in Table 4.1 (Chapter 4). PCR products from multiple loci were pool-plexed into a single 96-plate well (one set of products was comprised of four loci and another had five loci) based on the combinations recommended by the MULTIPLEX MANAGER 1.0 program (Holleley \& Geerts 2009). Capillary separation of the fluorescently labelled PCR-fragments was conducted on an ABI3730 Genetic Analyser (Massey University Genome Service). The allele sizes were scored using the AUTOBIN program (http://www4.bordeauxaquitaine.inra.fr/biogeco/Ressources/Logiciels/Autobin), which uses an Excel macro to sort and plot the relevant gaps between allele sizes (Guichoux et al. 2011). Whenever a new allele was detected in a mother and their offspring it was genotyped for second time to confirm the presence of a new allele. Although, it was not common to detect new alleles $(9.4 \%$ of all sharks screened) the data quality was checked to avoid genotyping errors. 


\subsubsection{Statistical analyses}

The microsatellite genotyping data of G. galeus collected from East of North Island (ENI, $n=$ 40) were pooled with the genotyping data obtained from pregnant females resulting in 45 individual used in this study. MICRO-CHECKER 2.2.3 (Van Oosterhout et al. 2004) was used to check for scoring errors and the presence of null alleles. Population genetic diversity indexes e.g. number of alleles per locus $(A)$, observed $\left(H_{\mathrm{o}}\right)$ and expected $\left(H_{\mathrm{e}}\right)$ heterozygosities, exact tests for departures from Hardy-Weinberg equilibrium (HWE), and tests for pairwise Linkage Disequilibrium (LD) between loci were calculated in ARLEQUIN 3.1.1 (Excoffier et al. 2005). HWE significance was calculated using the exact test (Guo \& Thompson, 1992) with a Markov chain of $10^{6}$ steps and $10^{5}$ dememorization steps. Significance values for LD were calculated using a likelihood-ratio test (LRT) of 20,000 permutations (Slatkin and Excoffier, 1996). Sequential Bonferroni corrections (Rice 1989) were applied when appropriate. Allele frequency distributions at each loci and progeny arrays and ENI population were generated using GENALEX 6.41 (Peakall \& Smouse 2006).

The probability to detect and quantify MP within an offspring depend in the polymorphism of the microsatellite loci used in the study, the litter, the number of putative fathers and their corresponding reproductive success (Neff \& Pitcher 2002). In order to compare the results of MP with other shark species, such as Negaprion brevirostris, Ginglymostoma cirratum, Sphyrna tiburo, Squalus acanthias and Scyliorhinus canicula (Feldheim et al. 2001, 2002, Chapman et al. 2004, Lage et al. 2008, Griffiths et al. 2012) two programs were used for the G. galeus multilocus genotypes data i.e. PRDM (Probability to detect multiple mating, Neff \& Pitcher 2002) and GERUD 2.0 (Jones 2005). PRDM was used to estimate the power of detecting MP at six microsatellite loci. PRDM calculates the probability of detecting a multiple mating (fertilization) using the allele frequency distribution of each locus for ENI population and five pregnant females. Following similar simulations used in Chapman et al. 2004 and Byrne \& Avise 2002, six scenarios for MP in $G$. galeus were tested: a) two males with equal breeding success (50\% each), b) two males with unequal breeding success $(66.7 \%$ and $33.3 \%)$, c) three males with equal breeding success (33.3\% each), d) three males with unequal success $(57 \%, 28.5 \%$ and $14.5 \%)$, e) four males with equal success (25\% each), f) four males with unequal breeding success (50\%, 27\%, 14\% and 7\%). Because the probability to detect MP mating is also in function of the number of embryos analysed, PRDM simulation were ran with litter size ranging from 15 to 52 which is 
the variation of the fecundity for G. galeus in Australasia region (Olsen 1954, 1984, Walker 2005 ) and it included the number of litters observed in this study (ranged from 23 to 34).

Two approaches were used for parentage analysis were carried out. Firstly, manual allele counting by constructing a locus genotype for each embryo in a spreadsheet data set. The observed maternal allele is known from genetic or behavioral evidence and paternally is in question. For each offspring at each locus, the maternal allele is inferred by subtraction for each locus in order to obtain its paternally derived alleles. The analysis is conducted by manual inspection of the dataset. The criterion to establish the occurrence of MP of a litter for both approaches was determined for the presence of more than two paternal alleles across at least two loci (Avise et al. 2002, Chapman et al. 2004, Lage et al. 2008). Secondly, the program GERUD 2.0 (Jones 2005) was used to reconstruct the parental multilocus genotypes from progeny arrays. This new version uses an exhaustive search algorithm to determine the minimum number of parents necessary to explain a given half-sib progeny array with one or neither parental known. This search involves three steps: a) establish the paternal alleles for each offspring by subtraction (approach is explained above); b) determine all combinations of alleles observed in the progeny array that yield paternal genotypes consistent with the array; and c) find the better combinations of paternal genotypes that sired the progeny array. In order to have some idea which solution is most probably, they were ranked by likelihood of a particular combination of fathers. Probability scores base on Mendelian segregation were estimated for the fifty combination of father with highest probabilities. Additionally, GERUD2.0 was used to calculate the expected exclusion probabilities based on the allele frequency of the population ENI. This calculation provides an indication of the power of the microsatellite loci used for parentage assessment. 


\subsection{Results}

There were no scoring problems or null alleles detected at the six microsatellite loci. The six microsatellite loci showed relatively high allelic diversity in the population sampled (ENI, $n$ $=40$ and pregnant females, $n=5$ ). The number of alleles found range from 5 alleles for $G g 7$ to 11 alleles for $G g 15$ (Table 5.1). Five of the six microsatellite loci screened in this study were found to be in HWE. Only loci $G g 11$ exhibited significant departures from HWE ( $P$ $<0.001)$ due to its deficit of heterozygotes observed. Pairwise combination for $G g 23$ and $G g 7$ loci showed a significant level of LD $(P<0.001)$. Exclusion probabilities from GERUD for each locus identified the majority of microsatellites were informative markers for parentage assessment with high values of combined (all loci) exclusion probability $(>0.94)$.

Table 5.1: Allelic frequency distribution and genetic diversities at six microsatellite loci for $45 G$. galeus sharks from New Zealand waters i.e. East of North Island (ENI) and mums collected in the present study. Number of sharks scored $\left(N_{\mathrm{i}}\right)$, Number of alleles $(\mathrm{A})$, observed heterozygosity $\left(H_{\mathrm{o}}\right)$, expected heterozygosity $\left(H_{\mathrm{e}}\right)$, and the probabilities of Hardy Weinberg Equilibrium $\left(P_{\mathrm{HWE}}\right)$. The asterisk $(*)$ means deviate from Hardy-Weinberg expectations nominally after sequential Bonferroni correction (alpha $=0.05 / 6=0.008)$. Exclusion probabilities $(E p)$ estimated in GERUD is presented by each locus and across loci.

\begin{tabular}{|c|c|c|c|c|c|c|c|c|c|c|c|c|}
\hline \multicolumn{2}{|c|}{$G g 15$} & \multicolumn{2}{|c|}{$G g 22$} & \multicolumn{2}{|c|}{$G g 23$} & \multicolumn{2}{|c|}{$G g 12$} & \multicolumn{2}{|l|}{$G g 7$} & \multicolumn{2}{|c|}{$G g 11$} & \\
\hline 152 & 0.037 & 240 & 0.023 & 264 & 0.202 & 284 & 0.083 & 296 & 0.022 & 334 & 0.136 & \\
\hline 154 & 0.061 & 242 & 0.488 & 266 & 0.155 & 286 & 0.036 & 298 & 0.200 & 350 & 0.455 & \\
\hline 156 & 0.024 & 244 & 0.012 & 268 & 0.083 & 288 & 0.357 & 308 & 0.156 & 354 & 0.159 & \\
\hline 158 & 0.098 & 246 & 0.023 & 270 & 0.381 & 290 & 0.226 & 310 & 0.533 & 358 & 0.011 & \\
\hline 160 & 0.122 & 248 & 0.163 & 272 & 0.071 & 292 & 0.119 & 312 & 0.089 & 374 & 0.045 & \\
\hline 162 & 0.159 & 250 & 0.279 & 274 & 0.060 & 294 & 0.083 & & & 378 & 0.193 & \\
\hline 164 & 0.024 & 260 & 0.012 & 276 & 0.036 & 296 & 0.036 & & & & & \\
\hline 168 & 0.244 & & & 278 & 0.012 & 298 & 0.048 & & & & & \\
\hline 170 & 0.183 & & & & & 310 & 0.012 & & & & & \\
\hline 172 & 0.012 & & & & & & & & & & & \\
\hline 174 & 0.037 & & & & & & & & & & & \\
\hline$N_{\mathrm{i}}$ & 41 & & 43 & & 42 & & 42 & & 45 & & 44 & \\
\hline A & 11 & & 7 & & 8 & & 9 & & 5 & & 6 & \\
\hline$H_{\mathrm{o}}$ & 0.829 & & 0.651 & & 0.690 & & 0.643 & & 0.667 & & 0.432 & \\
\hline$H_{\mathrm{c}}$ & 0.860 & & 0.663 & & 0.782 & & 0.798 & & 0.650 & & 0.718 & \\
\hline$P_{\mathrm{HWE}}$ & 0.355 & & 0.175 & & 0.409 & & 0.125 & & 0.029 & & $0.001 *$ & \\
\hline$E p$ & 0.871 & & 0.580 & & 0.772 & & 0.795 & & 0.592 & & 0.675 & $\begin{array}{l}\text { All loci } \\
1.000\end{array}$ \\
\hline
\end{tabular}


In general, the microsatellite loci provided a good level of power of detecting MP for the majority of the families (ranged from $99.2 \%$ to $100 \%$, Table 5.2). The probability to detect multiple mating increased with the litter size and the number of fathers. Considering this result and that litters studied comprised more than 20 embryos, the use of this six microsatellite loci allow to this study to make reliable inferences on the mating system of $G$. galeus in New Zealand waters. Exclusion probabilities from GERUD for each locus (Table 5.1) identify that the six microsatellite loci are informative to detect MP solution when more than one unique combination of fathers can explain the data.

Table 5.2: Probability of detecting MP (PRDM) values for six microsatellite loci used assuming six mating schemes and specific litter size for each Family and overall families.

\begin{tabular}{llllllll}
\hline \multicolumn{7}{l}{ Litter size } \\
\cline { 2 - 8 } $\begin{array}{l}\text { Mating schemes } \\
\text { (hypothesized paternal skew) }\end{array}$ & 15 & 23 & 25 & 29 & 34 & 40 & 52 \\
\hline 2 males (50:50) & 0.997 & 0.998 & 0.999 & 0.999 & 0.999 & 0.999 & 0.999 \\
2 males (66.7:33.3) & 0.992 & 0.998 & 0.998 & 0.999 & 0.999 & 0.999 & 0.999 \\
3 males (33.3:33.3:33.3) & 1.000 & 1.000 & 1.000 & 1.000 & 1.000 & 1.000 & 1.000 \\
3 males (57:28.5:14.5) & 0.999 & 1.000 & 1.000 & 1.000 & 1.000 & 1.000 & 1.000 \\
4 males (25:25:25:25) & 1.000 & 1.000 & 1.000 & 1.000 & 1.000 & 1.000 & 1.000 \\
4 males (50:27:14:7) & 1.000 & 1.000 & 1.000 & 1.000 & 1.000 & 1.000 & 1.000 \\
\hline
\end{tabular}


A minimum estimation of three sires and two sires were found in the Family 1 and Family2 based on the simple allele counting for the construction multilocus genotype (Table 5.3). All the rest of Families were considered to be single paternity genotypes. Despite of Family2 exhibited evidence of at least two sires at locus $G g 22$, the offspring are considered to be product of single paternity genotypes due to it was supported by one locus rather than all loci showing this pattern (e.g. Family1). Using the six microsatellite loci to estimate the minimum number of sires using GERUD resulted in genetic polyandry found in two of five progeny arrays (40\%, Table 5.4)

Table 5.3: Galeorhinus galeus Families (Mum = mother and Emb $=$ embryo) genotypes consistent with MP. Samples were genotyped uing all loci, but only the relevant genotypes are represented below. Paternal alleles are shown in bold by subtraction from the maternal alleles.

\begin{tabular}{|c|c|c|c|c|c|c|c|c|c|c|c|}
\hline Sample & $G g 15$ & Sample & $G g 22$ & Sample & $G g 23$ & Sample & $G g 12$ & Sample & $G g 7$ & Sample & $G g 11$ \\
\hline \multicolumn{12}{|l|}{ Family1 } \\
\hline Muml & 170,170 & Mum1 & 242,248 & Mum1 & 272,278 & Muml & 288,288 & Mum 1 & 310,310 & Mum1 & 350,350 \\
\hline Embl & 170,170 & Emb2 & $248, \mathbf{2 5 0}$ & Emb2 & $\mathbf{2 6 4}, 272$ & Emb13 & 288,298 & Emb2 & 310,312 & Emb2 & $\mathbf{3 5 0}, 350$ \\
\hline \multirow[t]{4}{*}{ Emb12 } & 154,170 & Emb3 & $\mathbf{2 4 2}, 242$ & Emb3 & 266, 278 & & & Emb3 & 310,310 & Emb3 & 350,354 \\
\hline & & Emb27 & 248,256 & Emb4 & $278, \mathbf{3 5 0}$ & & & & & Emb4 & $\mathbf{3 3 4}, 350$ \\
\hline & & & & Embl5 & 272,272 & & & & & & \\
\hline & & & & Emb26 & 268,272 & & & & & & \\
\hline \multicolumn{12}{|l|}{ Family2 } \\
\hline Mum2 & 152,170 & Mum2 & 244,250 & Mum2 & 266,272 & Mum2 & 290,290 & Mum2 & 310,312 & Mum2 & 350,350 \\
\hline Emb37 & 162,170 & Emb37 & $\mathbf{2 5 0}, 250$ & Emb37 & $\mathbf{2 7 2}, 272$ & Emb2 & $\mathbf{2 9 0}, 290$ & Emb37 & $\mathbf{3 1 2}, 312$ & Emb37 & $\mathbf{3 5 0}, 350$ \\
\hline \multirow[t]{2}{*}{ Emb40 } & $152, \mathbf{1 5 8}$ & Emb39 & $250, \mathbf{2 5 6}$ & & & & & Emb40 & $\mathbf{2 9 8}, 310$ & Emb39 & $\mathbf{3 4 6}, 350$ \\
\hline & & Emb53 & 244,248 & & & & & & & & \\
\hline \multicolumn{12}{|l|}{ Family3 } \\
\hline Mum3 & 168,168 & Mum3 & 242,250 & Mum3 & 270,272 & Mum3 & 284,290 & Mum3 & 310,312 & Mum3 & 350,350 \\
\hline Emb72 & 168,168 & Emb72 & $\mathbf{2 5 0}, 250$ & Emb37 & 270,270 & Emb72 & $\mathbf{2 8 4}, 284$ & Emb72 & $\mathbf{3 1 0}, 310$ & Emb72 & $\mathbf{3 5 0}, 354$ \\
\hline Emb73 & $\mathbf{1 5 4}, 168$ & Emb73 & $\mathbf{2 4 2}, 242$ & & & Emb73 & $284, \mathbf{3 0 0}$ & & & & \\
\hline \multicolumn{12}{|l|}{ Family 4} \\
\hline Mum4 & 152,170 & Mum4 & 242,250 & Mum4 & 264,264 & Mum4 & 288,294 & Mum4 & 310,312 & Mum4 & 334,354 \\
\hline Emb96 & 162,170 & Emb99 & $\mathbf{2 4 2}, 242$ & Emb97 & 264,270 & Emb72 & $288, \mathbf{2 9 0}$ & Emb97 & $\mathbf{3 1 0}, 310$ & Emb96 & $\mathbf{3 5 0}, 354$ \\
\hline Emb97 & $152, \mathbf{1 6 0}$ & & & Emb103 & 264,264 & & & Emb99 & $\mathbf{3 1 2}, 312$ & & \\
\hline \multicolumn{12}{|l|}{ Family 5} \\
\hline Mum5 & 158,168 & Mum5 & 242,250 & Mum5 & 268,272 & Mum5 & 290,310 & Mum5 & 298,310 & Mum5 & 350,350 \\
\hline Emb122 & $\mathbf{1 5 4}, 158$ & Emb122 & $\mathbf{2 4 2}, 242$ & Emb122 & 272,274 & Emb122 & $\mathbf{2 8 8}, 310$ & Emb122 & 298, 298 & Emb96 & $\mathbf{3 5 0}, 350$ \\
\hline Emb123 & 168,170 & Emb123 & $242, \mathbf{2 4 8}$ & & & Emb127 & $\mathbf{2 9 0}, 290$ & Emb123 & $298, \mathbf{3 0 8}$ & & \\
\hline
\end{tabular}


Table 5.4: Summary of analysed Families (mums and offspring) of G. galeus genotypes consistent with MP inferred in GERUD2.0. The number of sires (\# sires) were estimated by allele counting (AC) using a multilocus genotypes (see details Table 5.3), and GERUD. The number of solution (\# solutions), number of progeny (\# progeny) and the probability score (PS) were obtained from GERUD as well.

\begin{tabular}{lccll}
\hline & & Allele counting & GERUD & \\
\cline { 3 - 5 } & $\begin{array}{l}\text { Number of } \\
\text { offspring } \\
\text { genotyped }\end{array}$ & $\begin{array}{l}\text { Number } \\
\text { of sires }\end{array}$ & $\begin{array}{l}\text { Number } \\
\text { of sires }\end{array}$ & $\begin{array}{l}\text { Paternal } \\
\text { skew }\end{array}$ \\
\hline Families & 34 & 3 & 4 & $27 / 3 / 2 / 2$ \\
Family 1 & 34 & 2 & 2 & $19 / 15$ \\
Family 2 & 23 & 1 & 1 & 23 \\
Family4 & 25 & 1 & 1 & 25 \\
Family5 & 29 & 1 & 1 & 29 \\
\hline
\end{tabular}




\subsection{Discussion}

This study provides the first evidence of multiple paternity (MP) in G. galeus. In comparison to the previous studies that assessed paternity in elasmobranch i.e. 15 species in 18 studies, this study showed one of the lowest sample size of litter analyzed (five litters), but one of the largest average per litter size (22.8) across live-bearing shark species, compensating the likelihood to detect MP (Table 5.1 in Supplementary material). Most of the elasmobranch species have shown evidence of MP with exception of Carcharhinus galapagensis (DalyEngel et al. 2006) and Rhincodon typus (Schmidt et al. 2010), which the number of litters and number of progeny per litter were quite low in comparison other studies i.e. 1 litter by each species, C. galapagensis7 embryos and R. typus 29 embryos analysed. The observed range of 1-4 or 1-2 sires per offspring found in G. galeus is consistent with the same values among other genetically surveyed elasmobranch species. Parentage analyses demonstrated that female G. galeus showed evidence of polyandrous mating with different males during copulation events. In the present study, it was found that both methods used to detect MP i.e. allele counting vs. GERUD generated similar maternal and paternal locus genotypes reconstruction. Despite the fact that the number of litters sampled was low (five litters), the large number of embryos by offspring (22.8 average litter size; see Table 5.1 in Supplementary material) allowed detection of MP in at least two (40\%) of five litters of $G$. galeus. There were multiple sired by four males for Family1, and sired by two males for Family 2. The occurrence of MP in Family1 was strongly supported by four loci ( $G g$ 23, $G g 12, G g 11$, and $G g 22$ ) across the same individuals genotyped e.g. Emb2, Emb3, and Emb4 (see details Table 5.3). In contrast, the MP detected in Family2 was only distinguished by the Gg22 locus. While this may be questionable, the statistical power to detect MP in Family2 was high (83-100\%, Table 5.2) and simulations ran in GERUD, giving a good support for the suggestion that the paternal contribution was accurately detected. The result also suggested that polyandry and monandry are occurring with roughly equal frequency in G. galeus population in New Zealand waters where this species is comprised by single panmictic population (results Chapter 2 and 4).

Sexual segregation is a common behavioral characteristic among vertebrate species e.g. elasmobranch. Segregation patterns in elasmobranch may results differences between sexes as consequence of differential habitat use or different life-history stages (Wearmounth et al. 2012). Elasmobranch species are usually segregated according to age, sex, and reproductive stage (Grogan \& Lund 2004). For example, they can use different habitat that 
might vary in the prey availability e.g. Triakis semifasciata (Carlisle \& Starr 2009), Negaprion brevirostris (Morrisey \& Gruber 1993), Carcharhinus limbatus (Heupel \& Hueter 2002); and temperature e.g. Schroederichthys chilensis (Fariña \& Ojeda), Carcharodon carcharias (Robbins 2007), Carcharias taurus (Bansemer \& Bennett 2009), Urobatis halleri (Jirik \& Lowe 2012), and also G. galeus (Walker et al. 2008), where pregnant females at most stages of gestation are observed during the gestation period in warm waters of the Great Australian Bight and Eastern region of the south coast of Western Australia.

Tagging data and fishing surveys have provided evidence that movement of G. galeus in southern Australia is related to the reproductive cycles of this species. Pregnant females with large follicles in the ovary and several eggs without macroscopically visible embryos in the uterus are considered to be ovulating (Walker 2005). This can develop over period of several months during June-January. Overlapping with this time, it is also known that adult G. galeus in both sexes migrate to edge of the continental shelf for mating purposes during colder months i.e. July-September in South Australian and New South Wales waters (Olsen 1954, 1989, Walker 2005). Under these observations, it is possible that copulation in $G$. galeus population in Australia/New Zealand may occur a few months before the ovulation. For instance, copulation behaviour within $G$. galeus population is thought to occur five months before ovulation in western south Atlantic (Peres \& Vooren 1991). Consequently, they most likely have a mechanism to maintain and prolong sperm storage for subsequently fertilization.

In many animals females have the ability to store sperm in their reproductive tract. It is thought that the sperm storage has evolved because the optimal copulation time did not match with the optimal fertilization time or birthing habitat locaion (Birkhead \& Moller 1993). In elasmobranch species, the nidamental gland is the structure that provides the storage of sperm before the ovulation and enhances the opportunities for sperm competition and probability of successful fertilization (Pratt 1993, Pratt \& Tanaka 1994, Maruska et al. 1996, Fishelson \& Baranes 1998, Hamlett et al. 1998, Conrath \& Musick 2002, Storrie et al. 2008, Galíndez et al. 2010). The function of this structure for sperm storage has also been reported for G. galeus non-pregnant female (mature ovary) from (Peres \& Vooren 1991), and confirmed from smears of another non-pregnant female (immature ovary) from New Zealand waters (personal observation, see Figure 5.2 in Supplementary material). Under this scenario, it is not difficult to imagine that fertilization during repeated ovulations could be achieved by multiple males, and consequently multiple sired can be detected in the offspring. In fact, most 
of the elasmobranch species surveyed to the date exhibits levels of MP in the progenies analysed. Only two shark species have showed single paternity i.e. Carcharhinus galapagensis and Rhincodon typus (see \% litter with MP Table 5.1 in Supplementary material).

The length of reproductive cycle varies widely among elasmobranch species. Dasyatis species and Urobatis jamaicensis may have two or three reproductive cycles in a year, where species such Chlamydoselachus anguineus and G. galeus can have 3 years or longer. Females of the school shark G. galeus have a well-defined triennial parturition frequency (Peres \& Vooren 1991, Walker 2005). It means that one third of the mature females give birth every year and implies that the gestation period in G. galeus is highly synchronized with all phases from ovulation to birth (Peres \& Vooren 1991, Walker 2005). Therefore, all gravid females should typically contain embryos in the same stage of develop. Consequently, it would be expected that there is synchrony in the mean total length (TL) of embryos, resulting two different groups: one with eggs present in uteri without macroscopically visible embryos present ( $0 \mathrm{~mm}$ TL, $n=54$ pregnant females), and b) embryos macroscopically visible (approximately 150-300 $\mathrm{mm}$ TL, $n=65$ pregnant females) observed at a specific time. This observation is consistent with the mean embryo length observed in the present study that were obtained at different years but at same season i.e. later spring (November 2009 for Mum3) to summer (February 2009 for Mum1, 2 and January 2012 for Mum 4 and 5). The synchrony of the five G. galeus females for both pregnant conditions is illustrated in the Figure 5.1 in Supplementary material. This graph is shown that the mean TL of embryos from four females (Group 1) was consistent with pregnant condition expected, and it is also consistent with second pregnant condition of one female with embryos at full-term and prepared for parturition (Group2). Nevertheless, macroscopically visible embryos of $G$. galeus are present the 12 month period from February to January with most attain a size of more than $300 \mathrm{~mm}$ TL at full term (Walker 2005). Within shark species it is common that a population exhibits synchronous growth of embryos over a period of $\sim 12$ months, particularly among inhabiting water $<300 \mathrm{~m}$ such as Mustelus lenticulatus (Francis \& Mace 1980), Mustelus mento (Smale \& Compagno 1997), Mustelus manazo (Yamaguchi et al. 1997), Mustelus canis (Conrath \& Musick 2002), Mustelus antarcticus (Walker et al. 2007), and also previously reported for Galeorhinus galeus (Walker 2005).

There is a positive relationship between uterine fecundity or number of in utero embryos and maternal body length (Musick \&Bonfil 2005). It is though that as a female 
becomes larger this increment results in a larger space in the body cavity to maintain embryos. This linear positive relationship is reported in population of Sphyrna lewini (Chen et al. 1988), Squalus acanthias (Hanchet 1988), G. galeus (Olsen 1984, Peres \& Vooren 1991, Walker 2005). However, the linear regression values have generally low $r^{2}$, so it may not be a very accurate predictor of fecundity. Nevertheless, because there is a tendency that smallest pregnant females carried fewer embryos $(\mathrm{TL}=144.5 \mathrm{~cm} ; 17$ embryos $)$ than a largest females $(\mathrm{TL}=159 \mathrm{~cm} ; 41$ embryos), it is possible that MP might be driven by increasing fecundity. This understanding is based on the MP observed from two G. galeus females, which were the largest females sampled here (163.2 and $157.8 \mathrm{~cm}$ TL). However, further investigation and larger sampling size of pregnant females are needed to test the difference in litter size between monandrous (SP) and polyandrous litters (MP).

In summary, this study has shown that MP is common in the aplacental viviparous shark species G. galeus in New Zealand waters. This finding was consistent with many aspects of its reproductive biology, included prolonged breeding season and potential for sperm storage. The MP results may be a consequence of coactive mating behavior by males facilitated by a high male encounter rates experienced during breeding aggregations. However, future investigations should use larger sample size to more thoroughly understand this component of mating system in G. galeus population in New Zealand and Australian waters. 


\subsection{Appendices}

\section{Supplementary material}

Supplementary Table 5.1: Summarize of the genetic mating systems studies reported to the date in elasmobranch species classified by reproductive strategy (RS), which are indicated as oviparity $(\mathrm{O})$, aplacental viviparity (AV), and placental viviparity (PV). The number of microsatellite loci (No. of msat), number of litter (No. litter analysed), average number of litter size (Average litter size), number of progeny (No. progeny analysed), range of sires per litter, and percentage of litter with MP (\% litter with MP) are reported for each species. The asterisk indicates the only study that use MHC II $\alpha$ locus instead of microsatellites loci.

\begin{tabular}{|c|c|c|c|c|c|c|c|c|}
\hline Species & RS & $\begin{array}{l}\text { No. of } \\
\text { msat }\end{array}$ & $\begin{array}{l}\text { No. litter } \\
\text { analysed }\end{array}$ & $\begin{array}{l}\text { Average } \\
\text { litter size }\end{array}$ & $\begin{array}{l}\text { No. progeny } \\
\text { analysed }\end{array}$ & $\begin{array}{l}\text { Range of } \\
\text { sires per litter }\end{array}$ & $\begin{array}{l}\% \text { litter } \\
\text { with MP }\end{array}$ & Reference \\
\hline \multicolumn{9}{|l|}{ Selachii } \\
\hline Carcharhinus altimus & PV & 8 & 1 & 9 & 9 & 2 & 100 & Daly-Engel et. al. 2006 \\
\hline Carcharhinus galapagensis & PV & 8 & 1 & 7 & 7 & 1 & 0 & Daly-Engel et. al. 2006 \\
\hline Carcharhinus plumbeus & PV & 8 & 1 & 7 & 7 & 2 & 100 & Daly-Engel et. al. 2006 \\
\hline C. plumbeus & PV & 6 & 20 & 5.5 & 110 & $1-2$ & 40 & Daly-Engel et. al. 2007 \\
\hline C. plumbeus & PV & 5 & 20 & 9.4 & 187 & $1-4$ & 85 & Portnoy et. al. 2007 \\
\hline Galeorhinus galeus & AV & 6 & 5 & 22.8 & 145 & $1-4$ & 40 & This study \\
\hline Ginglymostoma cirratum & AV & $1 *$ & 1 & 1 & 32 & 4 & 100 & Saville et. al. 2002 \\
\hline G. cirratum & AV & 12 & 3 & 33.3 & 100 & $5-7$ & 100 & Heist et. al. 2011 \\
\hline Mustelus henlei & PV & 4 & 14 & 13.2 & 185 & $1-3$ & 93 & Byrne \& Avise 2011 \\
\hline M. antarcticus & AV & 8 & 29 & 11.3 & 328 & $1-4$ & 24 & Boomer et. al. 2013 \\
\hline M. lenticulatus & AV & 8 & 19 & 3.9 & 74 & $1-3$ & 13 & Boomer et. al. 2013 \\
\hline Negaprion brevirostris & PV & 3 & 1 & 1 & 11 & 3 & 100 & Feldheim et. al. 2001a \\
\hline N. brevirostris & PV & 9 & 97 & 7.3 & 707 & $1-4$ & 87 & Feldheim et. al. 2004 \\
\hline N. brevirostris & PV & 11 & 85 & 4.8 & 408 & $1-4$ & 81 & DiBattista et. al. 2008 \\
\hline Rhincodon typus & AV & 9 & 1 & 1 & 29 & 1 & 0 & Schmidt et. al. 2010 \\
\hline Scyliorhinus canicula & $\mathrm{O}$ & 12 & 13 & 11.5 & 150 & $1-4$ & 92 & Griffiths et. al. 2012 \\
\hline Sphyrna tiburo & AV & 4 & 22 & 8.5 & 188 & $1-3$ & 19 & Chapman et. al. 2004 \\
\hline Squalus acanthias & AV & 7 & 10 & 5 & 50 & $1-2$ & 30 & Lage et. al. 2008 \\
\hline S. acanthias & AV & 7 & 29 & $4-11$ & NR & $1-2$ & 17 & Verissimo et. al. 2011 \\
\hline S. mitsukurii & AV & 8 & 27 & 6.6 & 178 & $1-2$ & 11 & Daly-Engel et. al. 2010 \\
\hline \multicolumn{9}{|l|}{ Batoidea } \\
\hline Raja clavata & $\mathrm{O}$ & 5 & 4 & 43.3 & 173 & $4-6$ & 100 & Chevolot et. al. 2007 \\
\hline
\end{tabular}




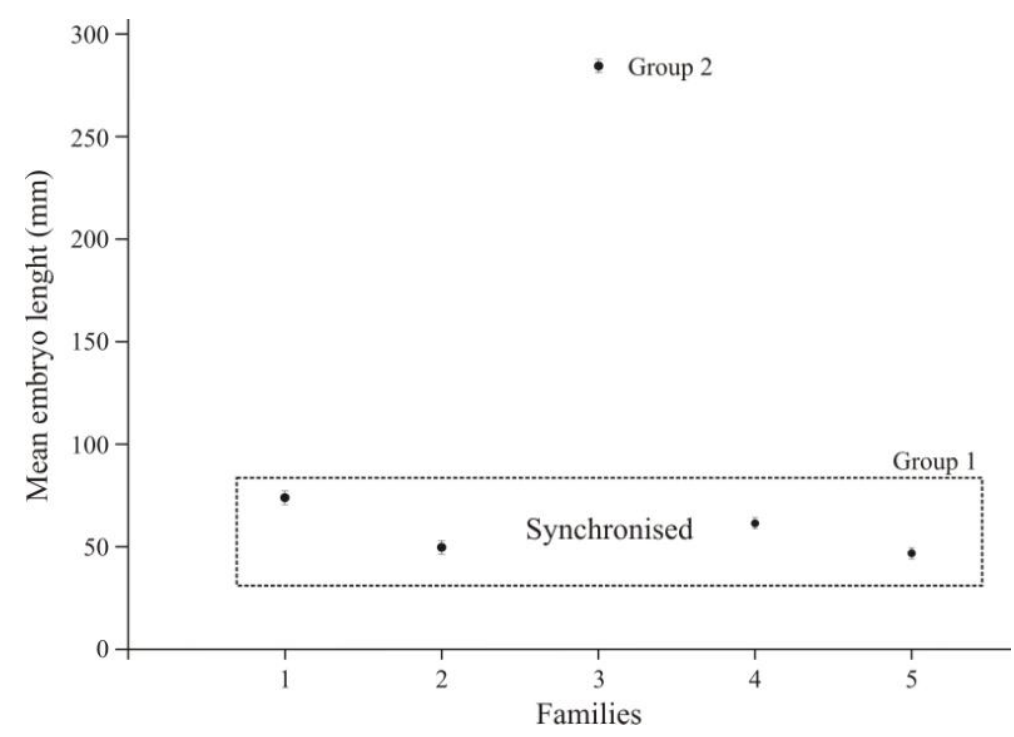

Supplementary Figure 5.1: Plot of the five progeny analysed based on the mean embryo total length (TL) of embryos collected from pregnant females.
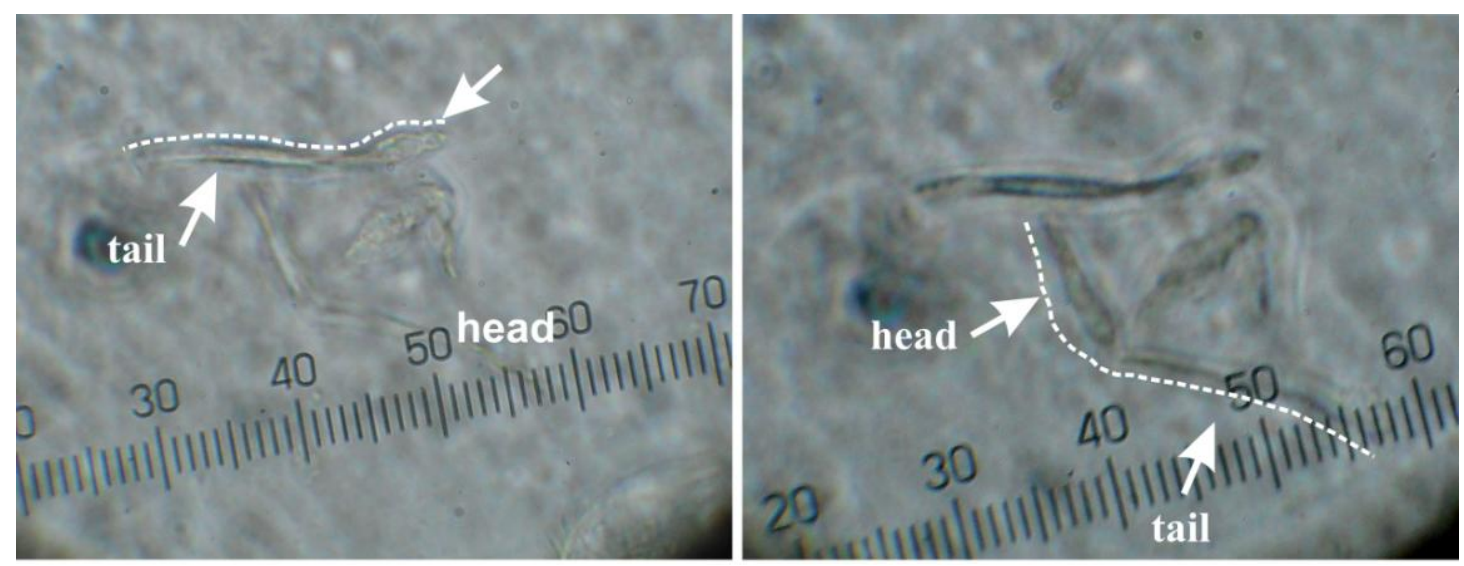

Supplementary Figure 5.2: Smears of nidamental gland of G. galeus no gravid with immature ovary. Both figures are the same but they are pointed different spermatozoa. The white dash line represents the orientation of each spermatozoa, and arrows indicate the head and tail of the spermatozoa. 


\section{Chapter 6}

\section{General Discussion}

\subsection{Genetic diversity and demographic history}

Population genetics has provided important insights for fisheries science, by understanding levels of genetic variation and the boundaries of reproductive units. Population genetic analyses have two main goals: to determine the level of genetic variation and the amount of differentiation among sample sites. Neutral molecular markers, such as mitochondrial DNA (mtDNA) and microsatellite DNA (msatDNA), are typically used because they can be applied across a range of species and the evolutionary mechanisms that influence these genomic regions are relatively well understood. The DNA sequence variation that is detected using mtDNA enables gene genealogies to be estimated and phylogeographic methods to be applied. Various mtDNA regions have been used for marine fish studies such as COI, ATPase, ND2, ND4, CytB, and CR. Most of the studies have concentrated in the CR because it often shows high levels of polymorphism compared to other mtDNA genes (Dudgeon et al. 2012). However, there are exceptions this generalisation about higher levels of diversity; other gene regions such as ND2 in Squalus acanthias (Veríssimo et al. 2010, O'Brien et al. 2013); and ND2, ATPase and COI in Stegostoma fasciatum (Dudgeon et al. 2009) can be more variable. In contrast, msatDNA markers show differences in the size of alleles because of differences in the number of repeat units of alleles. Genealogical methods are not directly applicable to microsatellite DNA markers, but they can have more statistical power because each locus is usually an independent sample of variation in a genomic region. Microsatellites have had some success when studies have attempted to amplification the same (or similar) loci in closely related species (Guichoux et al. 2011, Dudgeon et al. 2012). However, most population genetic studies of elasmobranch are based on species-specific microsatellite loci that were designed for each species (see list Table 3.1 in Supplementary Material, Chapter 3).

The patterns of genetic diversity that have been detected using mtDNA and msatDNA marks have shown similar result across different marine species. The findings were similar between marine teleost species (Actinopterygii) and Elasmobranch species (sharks and skates). These fish studies reported levels of variation based on methods that involved RFLPmtDNA, DNA sequencing of mtDNA regions (i.e. COI, CytB, and CR; Figure 6.1), and 
msatDNA (Figure 6.2). The results reported in Chapters 2 and 4, showed that the CR mtDNA and msatDNA had high levels of genetic diversity in G. galeus in comparison the other marine fish species. Based on the estimates of haplotype and nucleotide diversity for $\mathrm{CR}$ mtDNA, the genetic variation found in G. galeus $(h=0.79, \pi=0.0014)$ was higher than coastal shark species such Ginglymostoma. cirratum ( $h=0.16, \pi=0.0001$; Karl et al. 2012), Carcharhinus leucas ( $h=0.33, \pi=0.0006$; Karl et al. 2011), and Negaprion brevirostris $(h=$ $0.34, \pi=0.0004$; Schultz et al. 2008). However, this was lower than pelagic species such as Lamna ditropis ( $h=0.99, \pi=0.007$; O'Brien et al. 2013) and Rhincodon typus ( $h=0.95, \pi=$ 0.02; Ramírez-Marcías et al. 2007). In addition, similar patterns of genetic diversity in $G$. galeus were observed for the expected heterozygosity and allele richness $\left(H_{\mathrm{e}}=0.61, R_{S}=\right.$ 5.71) that were estimated using the microsatellite loci. The levels of genetic variation reported for G. galeus were higher than reported for Carcharhinus plumbeus $\left(H_{\mathrm{e}}=0.28, R_{S}=\right.$ 2.33; Heist \& Gold 1999), Carcharodon carcharias $\left(H_{\mathrm{e}}=0.48, R_{S}=7.00\right.$; Pardini et al. 2001), G. cirratum $\left(H_{\mathrm{e}}=0.58, R_{S}=5.83\right.$; Karl et al. 2002), but lower than Rhincodon typus $\left(H_{\mathrm{e}}=0.69, R_{S}=9.00 ;\right.$ Schmidt et al. 2009), $N$. brevirostris $\left(H_{\mathrm{e}}=0.79, R_{S}=28.50 ;\right.$ Feldheim et al. 2001), C. leucas $\left(H_{\mathrm{e}}=0.85, R_{S}=19.15\right.$; Karl et al. 2001), Isurus oxyrinchus $\left(H_{\mathrm{e}}=0.87\right.$, $R_{S}=25.25$; Schrey \& Heist 2003). Genetic diversity values were shown in Table 2.1 for CR mtDNA (Chapter 2) and Table 3.1 for msatDNA (Chapter 3) in Supplementary Material.

Interestingly, the differences between the genetic diversity values observed in a same species were extremely different when comparing both molecular marker (mtDNA and msatDNA). For instance, lower variability for CR mtDNA is found for Carcharhinus leucas, Negaprion brevirostris ( $h=0.33, \pi=0.001$ and $h=0.34, \pi=0.001$, respectively), whereas higher expected heterozygosity and allele richness were observed in both species $\left(H_{\mathrm{e}}=0.85\right.$, $R_{S}=19.15 ; H_{\mathrm{e}}=0.79, R_{S}=28.50$, respectively) (see Figure 6.1 and 6.2). The microsatellite loci screened in these species (C. leucas and N. brevirostris), and including Isurus oxyrinchus were characterised by higher number of motif repeat e.g. $(\mathrm{TG})_{19},(\mathrm{GT})_{20},(\mathrm{AC})_{33}$ that were highly polymorphic with a large allelic range (Schrey \& Heist 2003, Feldheim et al. 2001, Karl et al. 2011). This feature and the uninterrupted motif microsatellite enable more alleles to be distinguished and consequently improved the estimates of genetic diversity, resulting in different magnitude of genetic diversity when several loci are compared. This may also be attributed to differences in the modes of inheritance, where mtDNA is maternally inherited and nDNA is biparentally inherited. 
The estimates of genetic diversity also contains information about past population history. The mtDNA (e.g. CR, ND2, and CytB) and nDNA (microsatellite loci) markers were used to elucidate the demographic history of populations and compared to other species. Because mtDNA data is made up of discrete DNA sequence states and the molecule does not undergo recombination, it can be used to reconstruct major demographic events in recent population history (Heist 2005, Nielsen et al. 2009). For instance, a comparison can be made between the haplotype and nucleotide diversity estimated from CR mtDNA data and compared among shark species (see details Chapter 2, Table 2.1 and Figure 2.1 in Supplementary material). Three historical demographic categories can be described and these indicate the state of a population population size after the impacted in the contraction, if one occurred in recent history. Mitochondrial DNA analyses also allows for some insight into how distribution, habitat and behavior of species might have shaped the genetic patterns. The global glaciation events that occurred over the last 1.8 millions years are often suggested to have been a major influence on recent population history (Karl et al. 2011, O'Brien et al. 2013).

The Pleistocene was characterized by repeated glacial cycles separated by short interglacial periods (Lamberck et al. 2002). During glacial periods, marine systems experinced changes in temperature and sea levels were lower e.g. largest drop $130 \mathrm{~m}$ (Clark et al. 2009), which resulted in the reduction of coastal habitats and most likely large disruptions to the genetic connectivity among populations that was established during the interglacial periods. (Herbet et al. 2010) Phylogeographic studies using mtDNA have inferred much about the survival of population fragments in glacial refugia followed by post-glacial the expansion and secondary contact of populations and re-colonization of habitats (Hewitt 2004). The restriction of small populations to glacial refugia may have often led to high level of genetic differentiation and post-glacial secondary contact of these populations can be detected in modern population whenever a sample contains a mix divergent mtDNA lineages. For example, a recent phylogeographic analysis identified three distinct clades within populations Carcharhinus amboinensis, which was probably the result of isolation during the Pleistocene and continued restricted gene flow across contemporary northern Australia (Tillet et al. 2012). Two clades were found in Raja clavata from European populations suggesting that the British waters were a site of secondary contact after of repeated colonization from at least two ice-age refugia i.e. Iberian Peninsula and Azores (Chevolot et al. 2006). For G. galeus, the analyses of genetic data suggested that G. galeus in New Zealand/Australia 
population have historically been undergoing a period of expansion well before the postglacial warming and did not go through any severe population contraction during recent Pleistocene history. 


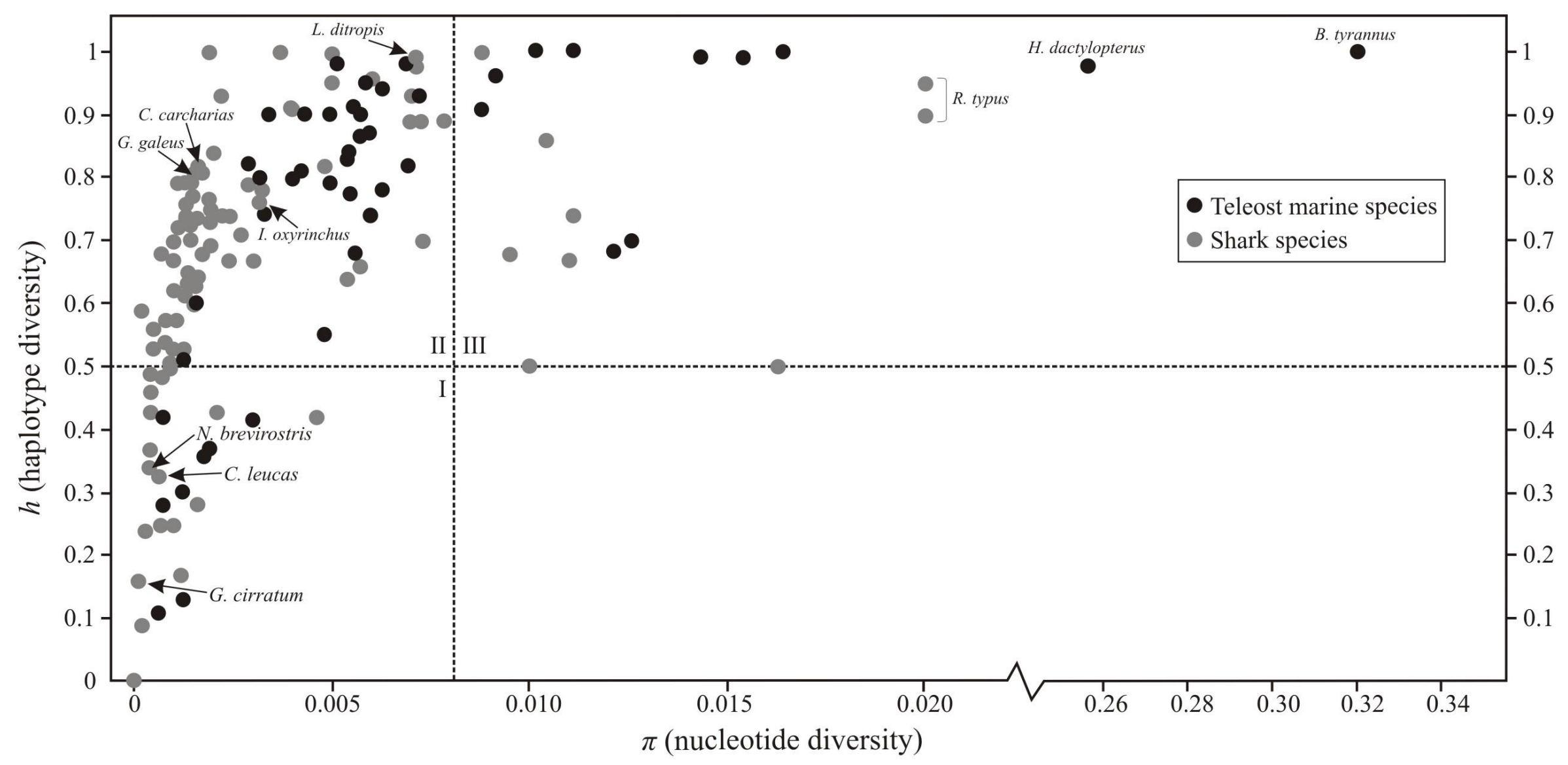

Figure 6.1: Relationship between the haplotypes $(h)$ and nucleotide diversity $(\pi)$ surveyed using RFLP-mtDNA, $C O I, C y t B$ and $C R$ of several population of teleost marine species (black circles) reported in Grant \& Waples 2000 and Varela 2012, and for population the CR sequences for 23 shark species (grey circles). The shark species labelled represents a particular population with high sample size (>30). 


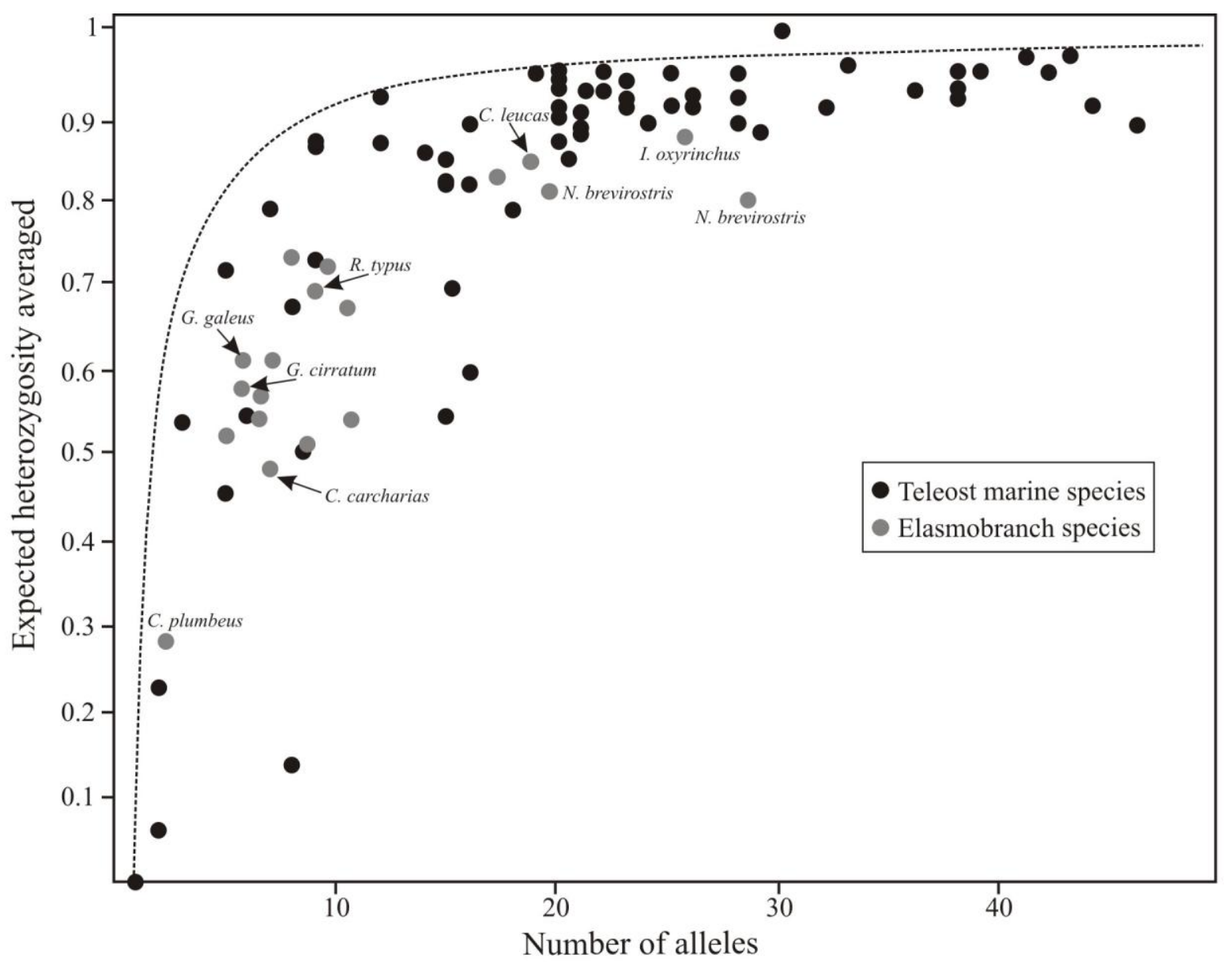

Figure 6.2: Relationship between the expected heterozygosity averaged and number of alleles surveyed using microsatellite loci of Teleost marine species (black circles) reported in DeWoody \& Avise 2000 and Varela 2012, and for 16 elasmobranch species (grey circles). The values of number of alleles and expected heterozygosity were obtained from Table 4.2 in Supplementary Material. The dash line showed the expected relationship of heterozygosity to the effective number of alleles reported in Figure 2 according to Crow \& Kimura (1970) in DeWoody \& Avise 2000. 


\subsection{Genetic differentiation and population structure}

Population genetic studies are usually implemented to assess the level of genetic connectivity among populations. Migrants moving and being reproductively successful with the residents at the new location determine level of population differentiation. Differences in gene frequencies among locations can be used indirectly to estimate patterns of gene flow and therefore the population structure of the species. For instance, the finding of genetic connectivity is more common in marine species than freshwater species because of a lack of barriers to migration in marine systems. A general conclusion is that population structure is particularly weak in highly mobile pelagic species (Waples 1998). The amount of genetic differentiation among populations is driven by population size, dispersal power, and the distance between the habitats occupied by different groups of individuals; genetic divergence is more often found in species that have limited amounts of suitable habitat that has a patchy distribution. Coastal shark species generally showed genetic differentiation across the large (but open) distances of oceans, but little or weak genetic differentiation along continental shelves.

Several examples of population genetic connectivity in shark species are discussed in Chapter 2 and 3 for CR mtDNA and nDNA (microsatellites). Based on that review of the literature, G. galeus was found to be a good example: populations from New Zealand and Australia are genetically connected across the Tasman Sea (see results Chapter 2 for mtDNA and Chapter 4 for nDNA). These results suggested that the G. galeus population has few barriers to reproductively successful migration, which was a finding consistent with their description of movements based on tagging studies (Olsen 1989, Hurst et al. 1999, Walker et al. 2008). However, New Zealand/Australian G. galeus populations appear to be separated across South Pacific Ocean and if there is any trans-Pacific migration it is does not result in reproductively successful migrants. It is important to note that the molecular markers have detected patterns of gene flow that have become established over long periods of time (thousands of years). If that pattern has recently changed (e.g. within the last 20-30 years) then it would be hard to detect without very large sample sizes or until the evolutionary process of migration led to more widespread genetic homogenization. Nevertheless, these results have given an important understanding of the level of population structure or genetic connectivity (opposite patterns) in G. galeus. 


\subsection{Fisheries management}

Strategies of management of living marine resource are strongly needed to ascertain the longterm maintenance of fisheries. A principal challenge for fisheries managers includes the development of strategies and decisions based on robust scientific evidence. The understanding of the life history and reproductive parameters are important for determining the best fisheries management response to a stock. Studies of stock structure are based on the notion of an ideal biological unit ('the stock') are usually determined using direct or indirect methods for detecting individual movements. Direct methods like surveys of egg and larva abundance, and tagging data (mark/recapture) provide are useful for understanding the movements in individual sharks (Kohler \& Turner 2001). However, these methods are unable to determine whether immigrants to a stock will survive and reproduce. For this reason, it is important the use of genetic markers that provide an indirect estimation of the relative reproductive contribution of migrants among stocks areas.

Molecular genetic approaches provide a powerful tool for addressing questions of genetic structure and connectivity of marine fishes. The use of these techniques have increased rapidly and given significant insight into fisheries stock structure. These studies are important because it is possible to identify stock boundaries, determine the spatial dimension of the stock (genetic connectivity), and describe the level of genetic diversity. Genetic diversity is important to manage because over the long-term is preserves evolutionary resilience, and provide the diversity, via gene flow, to other depleted stock (Carvalho \& Hauser 1994, Laikre et al. 2005). However, determining whether two stocks are from a single panmictic or from two separate stocks is not always easy and the results may not provide much assistance for management purposes.

Several fisheries management issues are often raised from genetic structure studies. When genetic differentiation between stocks has been detected there is a higher degree of certainty that both units are reproductively isolated. A difference can be easily detected using only a small sample size e.g. G. galeus samples from Chile $(N=16)$ was sufficient to detect genetic differentiation from New Zealand/Australia (Chapter II, Figure 5-20 individuals per population). This finding will be useful information for fishery managers. However, if no genetic differences are detected, it could be because either there is only one stock or there are two stocks that the genetic markers failed to detect. The uncertainty of this outcome is more to use for management purposes. There was uncertainty because: a) the type of genetic 
marker used may not have enough resolution to detect a difference when they are two separate stocks; b) the genetic difference between populations could have arisen only recently, which means the pattern cannot yet be seen in the genetic (Group II and III, Figure 6.3 ); or c) only a small amount of gene flow is necessary to homogenize genetic variation among populations, especially among large populations (e.g. marine species) where drift is weak and differentiation can be relatively low. Only a small numbers of migrants $(\mathrm{n}<10$, Waples 1998) are need to be reproduce successful after moving between New Zealand and Australia populations. At this point, the level of gene flow at different rates i.e. $1 \%, 5 \%, 10 \%$, $20 \%, 50 \%$ can be enough to show non-genetic differentiation (Group II, III. IV, Figure 6.3). However, those migration rates could have different consequences for stock assessment model. Consequently, fisheries genetics results have a degree of uncertain and may not be useful for fishery management purposes in some circumstances.

A lack of genetic differentiation at presumably neutral DNA markers such CR mtDNA and microsatellite loci, does not constitute proof of no genetic differentiation. The idea of a large and homogeneous panmictic stock between New Zealand and Australia is well supported for other sources of scientific information. The results of this thesis research were consistent with earlier genetic studies based on allozyme and RFLP-mtDNA and tagging data. However, The New Zealand and Australian G. galeus stock have so far been managed separately and assessment models used have estimates of spatial structure of the population derived from catch rates and tag /recapture data, the age-specific growth characteristics, pupping and recruitment processes, and the gear type employed in the fishery (Punt et al. 2000, 2005, MPI 2012). G. galeus is an example of a commercially species that straddles political and/or management boundaries with not joint management plan. Because administrative and logistic reasons school sharks in Australasia are considered as separate stocks. This is suggested to be more precautionary for G. galeus in Australia because if the assumption is made of one single stock, there is no reason to reduce the fishing rates in Australia (Waples et al. 2008). It is uncertain whether the regional management units are adequate for G. galeus; moreover the stock is depleted in Australia but the fishery is considered sustainable in New Zealand. Additionally, it is also concerning that the apparent sustainability of G. galeus biomass in New Zealand waters has been used to maintain of the high TAC levels through the last decade (>3000 t.). In Australian waters the historical nurseries areas have reduced and/or stopped to produce newborn young or pupping activity 
e.g. Port Phillip, Port Sorell, and St. Helen (Stevens \& West 1997), and most of the productive nurseries are in New Zealand waters.

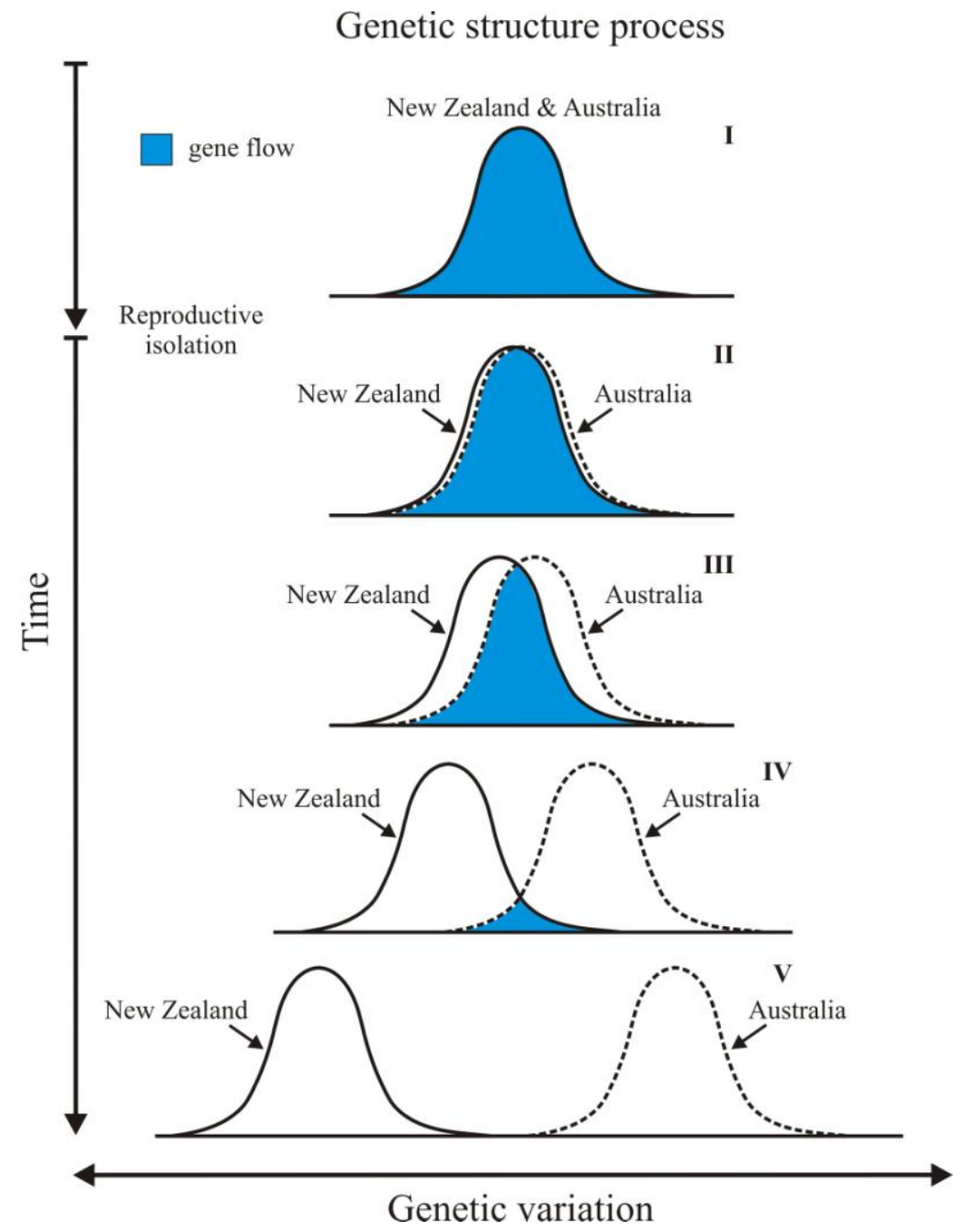

Figure 6.3: Stages of population structure process for G. galeus in New Zealand and Australia I. Single panmictic population of G. galeus in New Zealand and Australia; II. and III. More or less genetic variation start appear recently between New Zealand and Australia; IV. and V. New Zealand and Australia comprise of two genetically different stocks. Blue colour means the relative amount of gene flow. 


\subsection{Recommendation for Galeorhinus galeus management}

The new information presented in this thesis study is intended to inform management of $G$. galeus in the Australasian region. Following are four management considerations:

1. Genetic diversity ensures the evolutionary potential (ability to adapt to environmental changes) of the population and may buffer the shark population against climate change impacts. Stock sizes should be kept at current levels or higher to maintain the genetic diversity and genetic structure of New Zealand/Australian G. galeus. However, in New Zealand G. galeus stock size seem high and stable where in Australia the stock size is low and in collapse. Even though they are biologically one single stock - intermixing across geographical and political boundaries - the fishery status of the species is quite different in both countries, even in different part of each country. Therefore, they may need to be managed separately. For example, there are eight G. galeus management units (QMAs) in New Zealand that have separated TAC (total allowance catch). Even though, it is know that this species is a single stock in New Zealand, the TAC are allocated differentially in order to prevent any local depletion of G. galeus (e.g. protect aggregations of breeding females in Kaipara Harbour). In addition, treating New Zealand /Australia as a single stock is not practical because there would be no reason why to reduce the TAC in Australia. Consequently, different management approaches are needed.

2. The genetic information of a single biological stock should be integrated into the stock assessment models.

3. High-resolution genomic approaches (i.e. single nucleotide polymorphism, SNPs) should be considered for future genetic studies because they are able to detect gene flow and adaptive genetic variation. Genomics will enable functional differences (adaptive loci) of groups of individuals within the Australia/New Zealand stock to be detected. A detailed picture of the stock structure might enable a more precise set of area-specific management decisions.

4. Genetic monitoring of the New Zealand/Australian stocks should be conducted and emphasis should be put on sampling of neonates, pups, and juveniles. This approach will enable the genetic connectivity of different life stages to be 
determined and the degree of site fidelity to be properly defined. 


\section{References}

ABDELKRIM J, ROBERTSON, B, STANTON, J. A. \& GEMMELL, N. 2009. Fast, costeffective development of species-specific microsatellite markers by genomic sequencing. BioTechniques 46: 185-192.

AHONEN, H., HARCOUR, R. G. \& STOW, A. J. 2009. Nuclear and mitochondrial DNA reveals isolation of imperilled grey nurse shark populations (Carcharias taurus). Molecular Ecology 18: 4409-4421.

ALLENDORF, F. W., ENGLAND, P. R., LUIKART, G., RITCHIE, P. A. \& RYMAN, N. 2008. Genetic effects of harvest on wild animal population. Trends in Ecology and Evolution 26: 327-337.

ANDERSON, O. F., BAGLEY, N. W., HURST, R. J., FRANCIS, M. P., CLARK M. R. \& McMILLAN, P. J. 1998. Atlas of New Zealand fish and squid distributions from research bottom trawls. NIWA Technical Report No. 42. Wellington, New Zealand.

ANTAO, T., LOPES, A., LOPESM, R. J., BEJA-PEREIRA, A., LUIKART, G. 2008. LOSITAN: A workbench to detect molecular adaptation based on a $\mathrm{F}_{\mathrm{ST}}$-outlier method. BMC Bioinformatics 9: 1-5.

ARMOUR, J. A. L., NEUMANN, R., GOBERT, S. \& JEFFERY, A. J. 1994. Isolation of human simple repeat loci by hybridization selection. Human Molecular Genetics 3: 599605.

AUSTRALIAN FISHERIES MANAGEMENT AUTHORITY (AFMA). 2009. The school shark rebuilding strategy 2008. Available from http://www.afma.gov.au/wpcontent/uploads/2010/07/school_shark_rebuild.pdf

AUSTRALIAN FISHERIES MANAGEMENT AUTHORITY (AFMA). 2010a. Residual risk assessment of the level 2 ecological risk assessment species results report for the gillnet sector of the gillnet hook and trap fishery. Available from www.afma.gov.au/wpcontent/uploads/2010/06/rra_lvl2_psa.pdf

AUSTRALIAN FISHERIES MANAGEMENT AUTHORITY (AFMA). 2010b. South East management advisory committee SEMAC 2. TAC recommendations for the 2010/11 fishing season. Available from http://www.afma.gov.au/wp-content/uploads/2010/07/Jan28-item_234.pdf

AVISE, J. C., JONES, A. G., WALKER, D. \& DEWOODY, J. A. 2002. Genetic mating systems and reproductive natural histories of fishes: lessons for ecology and evolution. Annual Reviews of Genetics 36: 19-45.

BAGLEY, N. W., ANDERSON, O. F., HURST, R. J., FRANCIS, M. P., TAYLOR, P. R., CLARK, M. R. \& PAUL, L. J. 2000. Atlas of New Zealand fish and squid distributions from midwater trawls, tuna longline sets, and aerial sightings. NIWA Technical Report No. 72, Wellington, New Zealand.

BANSEMER, C. S. \& BENNETT, M. B. 2009. Reproductive periodicity, localised movements and behavioural segregation of pregnant Carcharias taurus at Wolf Rock, southeast Queensland, Australia. Marine Ecology Progress Series 374: 215-227. 
BAUM, J. K., MYERS, R. A., KEHLER, D. G, WORM, B., HARLEY, S. J. \& DOHERTY, P. A. 2003 Collapse and conservation of shark populations in the Northwest Atlantic. Science 299: 389-392

BEAUMONT, M. A. \& NICHOLS, R. A. 1996. Evaluating loci for use in the genetic analysis of population structure. Proceedings of the Royal Society of London Series B 263: $1619-1626$.

BELKHIR, K., BORSA, P., CHIKHI, L, RAUFASTE, N. \& BONHOMME, F. 2000. GENETIX 4.02, logiciel sous Windows TM pour la génétique des populations. Laboratoire Génome, Populations, Interactions, CNRS UPR 9060, Université de Montpellier II. Montpellier, France.

BENAVIDES, M. T., FELDHEIM, K. A., DUFFY, C. A., WINTNER, S., BRACCINI, J. M., BOOMER, J., HUVENEERS, C., ROGERS, P., MANGEL, J. C., ALFAROSHIGUETO, J., CARTAMIL, D. P. \& CHAPMAN, D. 2011. Phylogeography of the copper shark (Carcharhinus brachyurus) in the southern hemisphere: implications for the conservation of a coastal apex predator. Marine and Freshwater Research 62: 861-869.

BIRKHEAD, T. R. \& MOLLER, A. P. 1993. Sexual selection and the temporal separation of reproductive events: sperm storage data from reptiles, birds and mammals. Biological Journal Linnean Society 50: 295-311.

BRIGG, I. 1980. Upper Waitemata harbour catchment study. Working Report No. 17. Auckland, New Zealand.

BOOMER, J. J. \& STOW, A. J. 2010. Rapid isolation of the first set of polymorphic microsatellite loci from the Australian gummy shark, Mustelus antarticus and their utility across divergent shark taxa. Conservation Genetics Resources 3: 553-555.

BLOWER, D. C., PANDOLFI, J. M., GOMEZ CABRERA, M., BRUCE, B. D. \& OVENDEN, J. R. 2012. Population genetics of Australian white sharks reveals fine-scale spatial structure, transoceanic dispersal events and low effective population sizes. Marine Ecology Progress Series 455: 229-244.

BUONACCORSI, V. P., MCDOWELL, J. R. \& GRAVES, J. E. 2001. Reconciling patterns of inter-ocean molecular variance from four classes of molecular markers in blue marlin (Makaira nigricans). Molecular Ecology 10: 1179-1196.

BYRNE, R. J. \& AVISE, J. C. 2012. Genetic mating system of the brown smoothhound shark (Mustelus henlei), including a literature review of multiple paternity in other elasmobranch species. Marine Biology 159: 749-756.

CASPER, B. M., DOMINGO, A., GAIBOR, N., HEUPEL, M. R., KOTAS, E., LAMÓAMÓNACA, A. F., PÉREZ-JIMENEZ, J. C., SIMPFENDORFER, C., SMITH, W. D., STEVENS, J. D., SOLDO, A. \& VOOREN, C. M. 2005. Sphyrna zygaena. In: IUCN 2012. IUCN Red List of Threatened Species.

CAStOE, T. A, POOlE, A. W,. GU, W., JASON, A. P., DAZA, J. M., SMITH, E. \& POLLOCK, D. D. 2010. Rapid identification of thousands of copperhead snake (Agkistrodon contortrix) microsatellite loci from modest amounts of 454 shotgun genome sequence. Molecular Ecology Resources 10: 341-347. 
CASTRO, J. I., BUBUCIS, P. M. \& OVERSTROM, N. A. 1988. The reproductive biology of the chain dogfish, Scyliorhinus rotifer. Copeia 1988: 740-746.

CASTRO, A. L. F., STEWART, B. S., WILSON, S. G., HUETER, R. E., MEEKAN, M. G., MOTTA, P. J., BOWEN, B. W. \& KARL, S. A. 2007. Population genetic structure of Earth's largest fish, the whale shark (Rhincodon typus). Molecular Ecology 16: 51835192.

CARLISLE, A. B. \& STARR, R. M. 2009. Habitat use, residency, and seasonal distribution of female leopard sharks Triakis semifasciata in Elkhorn Slough, California. Marine Ecology Progress Series 380: 213-228.

CARRIER, J. C., PRATT, H. L. \& MARTIN, L. K. 1994. Group reproductive behaviors in free-living nurse sharks, Ginglymostoma cirratum. Copeia 1994: 646-656

CARRIER, J. C., PRATT, H. L. \& CASTRO, J. I. 2004. Reproductive biology of elasmobranchs. In: Carrier, J. C., Musick, J. A. \& Heithaus, M. R. (eds.) Biology of Sharks and their Relatives, CRC Press, Boca Raton, USA

CARVALHO, G. R. \& HAUSER, L. 1994. Molecular genetics and the stock concept in fisheries. Reviews in Fish Biology and Fisheries 4: 326-350.

CAVANAGH, R. D. \& LISNEY, T. J. 2003. Squalus mitsukurii. In: IUCN 2003.IUCN RedList of Threatened Species.

CHABOT, C. L. \& ALLEN, L. G. 2009. Global population structure of the tope (Galeorhinus galeus) inferred by mitochondrial control region sequence data. Molecular Ecology 18: 545-552.

CHABOT, C. L. \& NIGENDA, S. 2011. Characterization of 13 microsatellite loci for the tope shark, Galeorhinus galeus, discovered with next-generation sequencing and their utility for eastern Pacific smooth-hound sharks (Mustelus). Conservation Genetics Resources 2: 393-395.

CHABOT, C. L. 2012. Characterization of 11 microsatellite loci for the brown smooth-hound shark, Mustelus henlei (Triakidae), discovered with next-generation sequencing. Conservation Genetic Resources 4: 23-25.

CHAPMAN, D. D., CORCORAN, M. J., HARVEY, G. M., MALAN, S. \& SHIVJI, M. S. 2003. Mating behaviour of southern stingray, Dasyatis americana (Dsyatidae). Enviromental Biology of Fishes 68: 241-245.

CHAPMAN, D. D., PRODÖHL, P. A., GELSLEICHTER, J., MANIRE, C. A. \& SHIVJI, M. 2004. Predominance of genetic monogamy by females in a hammerhead shark, Sphyrna tiburo: implications for shark conservation. Molecular Ecology 13: 1965-1974.

CHEVOLOT, M., REUSCH, T. B. H., BOELE-BOS, S. \& STAM, W. T.\& OLSEN, J. L. 2005. Characterization and isolation of DNA microsatellite primers in Raja clavata L. (thornback ray, Rajidae). Molecular Ecology Notes 5: 427-429. 
CHEVOlOT, M., HOARAY, G., RIJNSDORSP, A. D., STAM, W. \& OLSEN, J. 2006. Phylogeography and population structure of thornback rays (Raja clavata L. Rajidae). Molecular Ecology 15: 3693-3705.

CHEVOLOT, M., ELLIS, J. R., RIJNSDORP, A. D., STAM, W. T. \& OLSEN, J. L. 2007. Multiple paternity analysis in the thornback ray Raja clavata L. Journal of Heredity 98: 712-715.

CORNUET, J. M. \& LUIKART, G. 1996. Description and power analysis of two tests for detecting recent population bottlenecks from allele frequency data. Genetics 144: 20012004.

CRAWFORD, N. G. 2010. SMOGD: software for the measurement of genetic diversity. Molecular Ecology Resources 10: 556-557.

CONRATH, C. L. \& MUSICK, J. A. 2002. Reproductive biology of the smooth dogfish, Mustelus canis, in the northwest Atlantic Ocean. Environmental Biology of Fishes 64: 367-377.

CSENCSINS, D., BRODBECK, S. \& HOLDEREGGER, R. 2010. Cost-effective, species specific microsatellite development for the endangered dwarf bulrush (Typha minima) using next-generation sequencing technology. Journal of Heredity 101: 789-793.

DALY-ENGEL, T. S., GRUBBS, E. D., HOLLAND, K. N., TOONEN, R. J. \& BOWEN, B. W. 2006. Assessment of multiple paternity in single litters from three species of carcharhinid sharks in Hawaii. Environmental Biology of Fishes 76: 419-424.

DALY-ENGEL, T. S., GRUBBS, R. D., BOWEN, B. W. \& TOONEN, R. J. 2007. Frequency of multiple paternity in an unexploited tropical population of sandbar sharks, Carcharhinus plumbeus. Canadian Journal of Fisheries and Aquatic Sciences 64: 198204.

DALY-ENGEL, T. S., GRUBBS, R. D, FELDHEIM, K. A., BOWEN, B. W. \& TOONEN, R. J. 2010. Is multiple mating beneficial or unavoidable? Low multiple paternity and genetic diversity in the shortspine spurdog Squalus mitsukurii. Marine Ecology Progress Series 403: 255-267.

DENT, E. A. \& BRIDGETT, V. M. 2012. Structure Harvester: a website and program for visualizing STRUCTURE output and implementing the Evanno method. Conservation Genetics Resources 4: 359-361.

DEPARTMENT OF SUSTAINABILITY, ENVIRONMENT, WATER, POPULATION AND COMMUNITIES (DSEWPaC). 2012. Galeorhinus galeus in Species Profile and Threats Database, Department of Sustainability, Environment, Water, Population and Communities, Canberra. Available from http://www.environment.gov.au/sprat

DEWOODY, J. A. \& AVISE, J. C. 2000. Microsatellite variation in marine, freshwater and anadromous fishes compared with other animals. Journal of Fish Biology 56: 461-473.

DIBATTISTA, J. D., FELDHEIM, K, A., THIBERT, X. P., GRUBER, S. H. \& HENRY, A. P. 2008. A genetic assessment of polyandry and breeding-site fidelity in lemon sharks. Molecular Ecology 17: 3337-3351. 
DICHMONT, C. M., OVENDEN, J. R. BERRY, O., WELCH, D. \& BUCKWORTH, R. C. 2012. Scoping current and future genetic tools, their limitations and their applications for wild fisheries management. CSIRO. Brisbane. Australia.

DIEMER, K. M., MANN, B, Q. \& HUSSEY, N. E. 2011. Distribution and movemenyt of scalloped hammerhead Sphyrna lewini and the smooth hammerhead Sphyrna zygaena sharks along the east coast of southern Africa. African Journal of Marine Science 33: 229-238.

DING, S. X., PAN, Y., ZENG, H. S. \& WANG, J. 2009. Characterization of 12 polymorphic microsatellite loci for the whitespotted bamboo shark (Chiloscyllium plagiosum Bennett). Molecular Ecology Resources 9: 1398-1400.

DI RIENZO, A., PETERSON, A. C., GARZA, J. C., VALDES, A. M., SLATKIN, M. \& FREIMER, N. B. 1994. Mutational processes of simple-sequence repeat loci in human populations. Proceedings of the National Academy of Sciences of the United States of America 91: 3166-3170.

DUDGEON, C. L., BRODERICK, D. \& OVENDEN, J. R. 2009. IUCN classification zones concord with, but undestimate, the population genetic structure of the zebra shark Stegostoma fasciatum in the Indo-West Pacific. Molecular Ecology 18: 248-261.

DUDGEON, C. L., BLOWER, D. C., BRODERICK, D. GILES, J. L., HOLMES,B. J., KASHIWAGI, T., KRÜCK, N. C., MORGAN, J. A. T., TILlETT, B. J. \& OVENDEN, J. R. 2012. A review of the application of molecular genetics for fisheries management and conservation of shark and rays. Journal of Fish Biology 80: 1789-1843.

DUFFY, C. A. J. \& LAST, P. R. 2007. Redescription of the northern spiny dogfish Squalus griffini Phillipps, 1931 from New Zealand. In: LAST, P. R., WHITE, W. T. \& POGONOSKI, J. J. (eds.) Descriptions of New Dogfishes of the genus Squalus (Squaloidea: Squalidae), CSIRO Marine and Atmospheric Research, Tasmania, Australia.

DUFFY, C. A. J. 2011. Squalus griffini. In: IUCN 2012. IUCN Red List of Threatened Species.

DUNCAN, K. M., MARTIN, A. P., BOWEN, B. W. \& DE COUET, H. G. 2006. Global phylogeography of the scalloped hammerhead shark (Sphyrna lewini). Molecular Ecology 15: 2239-2251.

DRUMMOND, A. J., RAMBAUT, A, DHAPIRO, B. \& PYBUS, O. G. 2005. Bayesian Coalescent inference of past population dynamics from molecular sequences. Molecular Biology and Evolution 22: 1185-1192.

DRUMMOND, A. J. \& RAMBAUT, A. 2007. Bayesian evolutionary analysis by sampling trees. BMC Evolutionary Biology 7: 214.

DRUMMOND, A. J., ASHTON, B., BUXTON, S., CHEUNG, M., COOPER, A., DURAN, C., FIELD, M., HELED, J., KEARSE, M., MARKOWITZ, S., MOIR, R., STONESHAVAS, S., STURROCK, S., THIERER, T. \& WILSON, A. 2011. Geneious v5.4. Available from http://www.geneious.com 
ELLEGREN, H. 2000. Microsatellite mutations in the germline: implications for evolutionary inference. Trends in Genetics 16: 551-558.

ELLEGREN, H. 2004. Microsatellites: simple sequences with complex evolution. Nature Reviews 5: 435-445.

ELY, B., VIÑAS, J., ALVARADO, J. R. B., BLACK, D., LUCAS, L., COVELlO, K., LABRIE, A. V. \& THELEN, E. 2005. Consequences of the historical demography on the global population structure of two highly migratory cosmopolitan marine fishes: the yellow tuna (Thunnus albacares) and the skipjack tuna (Katsuwonus pelamis). BMC Evolutionary Biology 5: 1-9.

ESTOUP, A., JARNE, P., CORNUET, J. M. 2002. Homoplasy and mutation model at microsatellite loci and their consequences for population genetics analysis. Molecular Ecology 11:1591-1604.

EXCOFFIER, L., SMOUSE, P. E. \& QUATTRO, J. M. 1992. Analysis of molecular variance inferred from metric distances among DNA haplotypes: application to human mitochondrial DNA restriction data. Genetics 131: 479-491.

EXCOFFIER, L., LAVAL, G. \& SCHNEIDER, S. 2005. Arlequin ver. 3.0: An integrated software package for population genetics data analysis. Evolutionary Bioinformatics Online 1: 47-50.

EVANNO, G., REGNAUT, S. \& GOUDET, J. 2005. Detecting the number of clusters of individuals using the software STRUCTURE: a simulation study. Molecular Ecology 14: 2611-2620.

FAIRCLOTH, B. C. 2008. MSATCOMMANDER: detection of microsatellite repeat arrays and automated, locus-specific primer design. Molecular Ecology Resources 8: 92-94.

FALUSH. D., STEPHENS, M. \& PRITCHARD, J. K. 2003. Inference of population structure using multilocus genotype data: linked loci and correlated allele frequencies. Genetics 4: 1567-1587.

FAO. 2012. The state of world fisheries and Aquaculture. FAO Fisheries and Aquaculture Department. Rome, Italy.

FARIÑA, J. M. \& OJEDA, P. 1993. Abundance, Activity, and trophic patterns of the redspotted catshark, Schroederichthys chilensis, on the Pacific temperate coast of Chile. Copeia 2: 545-549.

FELDHEIM, K. A., GRUBER, S. H. \& ASHLEY, M. V. 2001a. Multiplepaternity of a lemon shark litter (Chondricthyes: Carcharhinidae). Copeia 2001: 781-786.

FELDHEIM, K. A., GRUBER, S. H. \& ASHLEY, M. V. 2001b. Population genetic structure of the lemon shark (Negaprion brevirostris) in the western Atlantic: DNA microsatellite variation. Molecular Ecology 10: 295-303.

FELDHEIM, K. A., GRUBER, S. H. \& ASHLEY, M. V. 2004. Reconstruction of parental microsatellite genotypes reveals female polyandry and philopatry in the lemon shark, Negaprion brevirostris. Evolution 58: 2332-2342. 
FELDHEIM, K. A., STOW, A. J., AHONEN, H., CHAPMAN, D. D., SHIVJI, M., PEDDEMORS, V. \& WINTER, S. 2007. Polymorphic microsatellite markers for studies of the conservation and reproductive genetics of imperilled sand tiger sharks (Carcharias taurus). Molecular Ecology Notes 7: 1366-1368.

FELSENSTEIN, J. 1992. Estimating effective population size from samples of sequences: inefficiency of pairwise and segregating sites as compared to phylogenetic estimates. Genetic Resources 59: 139-147.

FISHELSON, L. \& BARANES, A. 1998. Observations on the oman shark, Iago omanensis (Triakidae), with emphasis on the morphologivcal and cytological changes of the oviduct and yolk sac during gestation. Journal of Morphology 236: 151-165.

FITZPATRICK, S., SHIVJI, M. S., CHAPMAN, D. D. \& PRODÖHL. 2011. Development and characterization of 10 polymorphic microsatellite loci for the blue shark, Prionace glauca, and their cross shark-species amplification. Conservation Genetic Resources 3: 523-527.

FITZPATRICK, S., KEMPSTER, R. M. DALY-ENGEL, T. S., COLLIN, S. P. \& EVANS, J. P. 2012. Assessing the potential for post-copulatory sexual selection in elasmobranchs. Journal of Fish Biology 80: 1141-1158.

FRANCIS, M. P. \& MACE, J. T. 1980. Reproductive biology of Mustelus lenticulatus from Kaikoura and Nelson. New Zealand Journal of Marine and Freshwater Research 14: 303311.

FRANCIS, M. P. \& MULLIGAN, K. P. 1998. Age and growth of New Zealand school shark, Galeorhinus galeus. New Zealand Journal of Marine and Freshwater Research 32: $427-$ 440.

FRANCIS, M. P. 1998. New Zealand shark fisheries: development, size and management. Marine Freshwater Research 49: 579-591.

FRANCIS, M. P. 2010. Movement of tagged rig and school shark among QMAs, and implications for stock management boundaries. New Zealand Fisheries Assessment Report 2010/3. Wellington, New Zealand,

FRANCIS, M. P. 2010. Review of research and observer data on hammerhead sharks (Sphyrna zygaena). Final Research Report IPA2009-01. Wellington, New Zealand.

FRANKHAM, R., BALLOU, J. D. \& BRISCOE, D. A. 2002. Introduction to Conservation Genetics. Cambridge University Press, Cambridge, UK.

FRANKHAM, R. 2005. Genetics and extinction. Biological Conservation 126: 131-140.

FU, Y. X. 1997. Statistical tests of neutrality of mutations against population growth, hitchhiking and background selection. Genetics 147: 915-925.

GALÍNDEZ, E. J. DÍAZ-ANDRADE, M. C., AVACA, M. S. \& ESTECONDO, S. 2010. Morphological study of the oviductal gland in the smallnose fanskate Sympterygia bonapartii (Müller and Henle, 1841) (Chondrichthyes, Rajidae). Brazilian Journal of Biology 70: 325-333. 
GARDNER, M. G., FITCH, A. J., BERTOZZI, T. \& LOWE, A. J. 2011. Rise of the machines-recommendations for ecologists when using next generation sequencing for microsatellite development. Molecular Ecology Resources 11: 1093-1101.

GARZA, J. C. \& WILLIAMSON, E. G. 2001. Detection of reduction in population size using data from microsatellite loci. Molecular Ecology 10: 305-318.

GORDON, I. 1993. Pre-copulatory behaviour of captive sand tiger sharks, Carcharias taurus. Environmental Biology of Fishes 38: 159-164.

GOUDET, J, 1995. FSTAT (vers. 2.9.3.2): a computer program to calculate F-statistics. Journal of Heredity 86: 485-486.

GOUDET, J., RAYMOND, M., MEEÜS, T. \& ROUSSET, F. 1996. Testing differentiation in diploid populations. Genetics 144: 1931-1938.

GOUDET, J. 1999. PCAGEN software for windows V.1.2.1. Available from http://www.2.unil.ch/popgen/softwares/pcagen.htm

GUICHOUX, E., LAGACHE, L., WAGNER, S., CHAUMEIL, P., LÉGER, P., LEPAIS, O., LEPOITTEVIN, C., MALAUSA, T., REVARDEL, E., SALIN, F. \& PETIT, R. J. 2011. Current trends in microsatellite genotyping. Molecular Ecology Resources 4: 591-611.

GUINDON, S., DUFAYARD, J. F., LEFORT, V., ANISIMOVA, M., HORDIJK, W. \& GASCUEL, O. 2010. New algorithms and methods to estimate maximun-likelihood phylogenies: assessing the performance of PhyML 3.0. Systematic Biology 59: 307-321.

GUO, S. W. \& Thompson, E. A. 1992. Performing the exact test of Hardy-Weinberg proportion for multiple alleles. Biometrics 48: 361-372.

GRANT, C. J., SANDLAND, R. L. \& OLSEN, A. M. 1979. Estimation of growth, mortality and yield per recruit of the Australian school shark, Galeorhinus australis (Macleay), from tag recoveries. Australian Journal of Marine and Freshwater Research 30: 625-637.

GRANT, W. S \& BOWEN, B. W. 1998. Shallow population histories in deep evolutionary lineages of marine fishes: insights from sardines and anchovies and lessons for conservation. The American Genetic Association 89: 415-426.

GRANT, W. S. \& WAPLES, R. S. 2000. Spatial and temporal scales of genetic variability in marine and anadromous species: Implications for fisheries oceanography. In: HARRISON, P. J. \& PARSONS, T. (eds.) Fisheries oceanography: a science for the new millennium, Blackwell Science, Oxford, UK.

GRAVES, J. E. 1998. Molecular insights into the population structures of cosmopolitan marine fishes. Journal of Heredity 89: 427-437.

GRIFFITHS, A. M., CASANE, D., MCHUGH, A., WEARMOUTH, V. J., SIMS, D. W. \& GENNER, M. J. 2011. Characterisation of polymorphic microsatellite loci in the smallspotted catshark (Scyliorhinus canicula L.). Conservation Genetic Resources 3: 705-709.

GRIFFITHS, A. M., JACOBY, D. M., CASANE, D., MACHUGH, M. CROFT, D. P., GENNER, M. J. \& SIMS, D. W. 2012. First analysis of multiple paternity in an oviparous shark, the small-spotted catshark (Scyliorhinus canicula L.). Journal of Heredity 2: 166173. 
GROGAN, E. D. \& LUND, R. 2004. The origin and relationships of early Chondrichthyes. In: CARRIER, J. C., MUSICK, J. A. \& HEITHAUS, M. R. (eds) Biology of sharks and their relatives. Boca Ratton: Florida, USA.

GUICHOUX, E., LAGACHE, L., WAGNER, S., CHAUMEIL, P., LÉGER, P., LEPAIS, O. LEPOITTEVIN, C., MALAUSA, T., REVARDEL, E., SALIN, F. \& PETIT, R. J. 2011. Current trends in microsatellite genotyping. Molecular Ecology Resources 11: 591-611.

GUINDON, S., DUFAYARD, J. F., LEFORT, V., ANISIMOVA, M., HORDIJK, W. \& GASCUEL, O. 2010. New algorithms and methods to estimate maximum-likelihood phylogenies: assessing the performance of PhyML 3.0. Systematic Biology 59: 307-321.

GUO, S. W. \& THOMPSON, E. A. 1992. Performing the exact test of Hardy-Weinberg proportion for multiple alleles. Biometrics 48: 361-372.

HAMILTON, M, PINCUS, E, DI FIORE, A. \& FLEISCHER, R. 1999. Universal linker and ligation procedures for construction of genomic DNA libraries enriched for microsatellites. BioTechniques 27: 500-506.

HAMLETT, W. C., KNIGHT, D. P., KOOB, T. J., JEZIOR, M., LUONG, T., ROZYCKI, T., BRUNETTE, N. \& HYSELL, M. K. 1998. Survey of oviductal gland structure and function in Elasmobranchs. The Journal of Experimental Zoology 282: 399-420.

HALE, M. L., BURG, T, M. \& STEEVES, T. E. 2012. Sampling for microsatellite-based population genetic studies: 25 to 30 individuals per population is enough to accurately estimate allele frequencies. PLOS ONE 7: 1-10.

HARPENDING, H. 1994. Signature of ancient population growth in a low resolution mitochondrial DNA mismatch distribution. Human Biology 66: 591-600.

HAUSER, L., FRANKS, J. H., VEGA, N. \& GALLUCCI, V. 2007. Potential for sustainable expansion of the dogfish (Squalus acanthias) fishery in the northeast Pacific. Final Report NOAA. Seattle, USA.

HAUSER, L. 2009. The molecular ecology of dogfish sharks. In: GALLUCCI, V. F., MVFARLANE, G. A, \& BARGMANN, G. G. (eds.) Biology and Management of Dogfish Sharks. American Fisheries Society, Bethesda, Maryland. USA.

HEDRICK, P. W. 2005. Genetics of populations. Jones \& Bamp Bartlett Publishers, Sudbury, Massachusetts, USA.

HEIST E. J., GRAVES, J. E \& MUSICK, J. A. 1995. Genetic population of the sandbar shark (Carcharhinus plumbeus) in the Gulf of Mexico and Mid-Atlantic Bight. Copeia 1995: 555-562.

HEIST E. J., MUSICK, J. A. \& GRAVES, J. E. 1996a. Genetic population structure of the shortfin mako (Isurus oxyrhinchus) inferred from restriction fragment length polymorphism analysis of mitochondrial DNA. Canadian Journal of Fisheries and Aquatic Science 53: 583-588.

HEIST, E. J., MUSICK, J. A. \& GRAVES, J. E. 1996b. Mitochondrial DNA diversity and divergence among sharpnose sharks, Rhizoprionodon terraenovae, from the Gulf of Mexico and Mid-Atlantic Bight. Fishery Bulletin 94: 664-668. 
HEIST, E. J. \& GOLD, J. R. 1999. Microsatellite DNA variation in sandbar sharks (Carcharhinus plumbeus) from the Gulf of Mexico and Mid-Atlantic Bight. Copeia 1: 182-186.

HEIST, E. J., JENKOT, J. L., KEENEY, D. B., LANE, R. L., MOYER, G. R., READING, B. J. \& SMITH, N. L. 2003. Isolation and characterization of polymorphic microsatellite loci in nurse shark (Ginglymostoma cirratum). Molecular Ecology Notes 3: 59-61.

HEIST, E. J. 2005. Genetics: stock identification. In: MUSICK, J. A. \& BONFIL, R (eds.) Management techniques for Elasmobranch. FAO Fisheries Technical Paper 474. Rome, Italy.

HEIST, E. J. 2009. Molecular markers and genetic population structure of pelagicsharks. In: CAMHI, M. D., PIKITCH, E. K. \& BABCOCK, E. A (eds.) Shark of the Open Ocean Biology, Fisheries and Conservation, Blackwell Publishing, Victoria, Australia.

HELYAR, S., COSCIA, I., SALA-BOZANO, M. \& MARIANI, S. 2011. New microsatellite loci for the longnose velvet dogfish Centroselachus crepidater (Squaliformes: Somniosidae) and other deep sea sharks. Conservation Genetic Resources 3: 173-176.

HERNANDEZ, S., HAYE, P. \& SHIVJI, M. 2008. Characterization of the pelagic shark fin trade in north central Chile by genetic identification and trader surveys. Journal of Fish Biology 73: 2293-2304.

HOLlELEY, C. E. \& GEERTS, P. G. 2009. Multiplex Manager 1.0: a cross-platform computer program that plans and optimizes multiplex PCR. Biotechniques 46: 511-517.

HUBISZ, M. J., FALUSH, D., STEPHENS, M. \& PRITCHARD, J, K. 2009. Inferring weak population structure with the assistance of sample group information. Molecular Ecology Resources 9: 1322-1332.

HURST, R. J., BAGLET, N. W., MCGREGOR, G. A. \& FRANCIS, M. P. 1999. Movements of the New Zealand school shark, Galeorhinus galeus, from tag returns. New Zealand Journal of Marine and Freshwater Research 22: 29-48.

HOELZEL, A. R., SHIVJI, M, S., MAGNUSSEN, J. \& FRANCIS, M. P. 2006. Low worldwide genetic diversity in the basking shark (Cetorhinus maximus). Biology Letters 2: 639-642.

HUETER, R. E., HEUPEL, M. R., HEIST, E. J. \& KEENEY, D. B. 2005. Evidence of phylopatry in sharks and implications for the management of shark fisheries. Journal of Northwest Atlantic Fishery Science 35: 239-247.

HURST, R. J., BAGLEY, N. W., ANDERSON, O. F., FRANCIS, M. P., GRIGGS, L. H., CLARK, M. R., PAUL, L. J. \& TAYLOR, P. R. 2000. Atlas of juvenile and adult fish and squid distributions from bottom and midwater trawlsand tuna longlines in New Zealand waters. NIWA Technical Report 84, Wellington, New Zealand.

IHAKA, R. \& GENTLEMAN, R. 1996. R: a language for data analysis and graphics. Journal of Computational and Graphical Statistics 5: 299-314

JOBLING, M., HOLLOX, E., KIVISILD, T., TYLER, C. S. \& HURLES, M. 2004. Human evolutionary genetics: Origins, people and disease. Garland Science, New York, USA. 
JORGENSEN, S. J., REEB, C. A., CHAPPLE, T. K., ANDERSON, S., PERLE, C., VAN SOMMERAN, S. R., FRITZ-COPE, C., BROWN, A, C., KLIMLEY, A. P. \& BLOCK, B. A. 2010. Phylopatry and migration of Pacific white sharks. Proceedings of the Royal Society B 277: 679-688.

JONES, A. G. 2005. GERUD 2.0: a computer for the reconstruction of parental genotypes from half-sib progeny arrays with known or unknown parents. Molecular Ecology Notes 5: 708-711.

JOST, L. 2008. $G_{\mathrm{ST}}$ and its relatives do not measure differentiation. Molecular Ecology 17: 4015-4026.

KARL, S. A., CASTRO, A. L. F., LOPEZ, J. A., CHARVET, P. \& BURGES, G. H. 2011. Phylogeography and conservation of the bull shark (Carcharhinus leucas) inferred from mitochondrial and microsatellite DNA. Conservation Genetics 12: 371-382.

KARL, S. A., CASTRO, A. L. F. \& GARLA, R. C. 2012. Population genetics of the nurse shark (Ginglymostoma cirratum) in the western Atlantic. Marine Biology 159: 489-498.

KASHIWAGI, T., BRODERICK, D., LANCE, S., BENNETT, M. B. \& OVENDEN, J. R. 2012. Development and characterization of ten microsatellite loci of the reef manta ray Manta alfredi. Conservation Genetic Resources 4: 1055-1058.

KEENEY, D. B. \& HEIST, E. J. 2003. Characterization of microsatellite loci isolated from the blacktip shark and their utility in requiem and hammerhead sharks. Molecular Ecology Notes 3: 501-504.

KEENEY, D. B., HEUPEL, M., HUETER, R. E. \& HEIST, E. J. 2005. Microsatellite and mitochondrial DNA analyses of the genetic structure of blacktip shark (Carcharhinus limbatus) nurseries in the northwestern Atlantic, Gulf of Mexico, and Caribbean Sea. Molecular Ecology 14: 1911-1923.

KEENEY, D. B. \& HEIST, E. J. 2006. Worldwide phylogeography of the blacktip shark (Carcharhinus limbatus) inferred from mitochondrial DNA reveals isolation of western Atlantic populations coupled with recent Pacific dispersal. Molecular Ecology 15: 36693679 .

KIMURA, M. \& CROW, J. F. 1964. The number of allele that can be maintained in a finite population. Genetic 49: 725-738.

KIMURA, M \& OHTA T. 1978. Stepwise mutation model and distribution of allelic frecuencies in a finite population. Procceding of Natural Academy of Science 75: 28682872.

KIMURA, M. 1989. The neutral theory of molecular evolution and the world view of the neutralists. Genome 31: 24-31.

KOHLER, N. E. \& TURNER, P. A. 2001. Shark tagging: a review of conventional methods and studies. Environmental Biology of Fishes 60: 191-223.

KROUGH, M. 1994. Spatial, seasonal and biological analysis of sharks caught in the New South Wales protective beach meshing programme. Australian Journal of Marine and Freshwater Research 45: 1087-1106. 
LAGE, C. R., PETERSEN, C. W., FOREST, D., BARNES, D., KORNFIELD, I. \& WRAY, C. 2008. Evidence of multiple paternity in spiny dogfish (Squalus acanthias) broods based on microsatellite analysis. Journal of Fish Biology 73: 2068-2074.

LAIKRE, L., PALM, S. \& RYMAN, N. 2005. Genetic population structure of fishes: implications for coastal zone management. A Journal of the Human Environment 34: 111119.

LAMILlA, J., ACUÑA, E., ARAYA, M., KONG, I., OLIVA, M., HERNANDEZ, S., VILlAROEL, J. C., CONCHA, F., VOGLER, R., PEQUEÑO, G. \& MUTSCHKE, E. 2005. Lineamientos basicos para desarrollar el plan de accion nacional de Tiburones. Informe Tecnicos FIP. FIP/ILN ${ }^{\circ}$ 2004-18. Valdivia, Chile.

LANGELlA, O. (2002). Populations 1.2.32. Logiciel de génétique des populations. Available from http://www.bioinformatics.org/project/?group_id=84.

LARSON, S., TINNEMORE, D. \& AMEMIYA, C. 2009. Microsatellite loci within sixgill sharks, Hexanchus griseus. Molecular Ecology Resources 9: 978-981.

LAST, P. R. \& STEVENS, J. D. 2009. Sharks and Rays of Australia. CSIRO Publishing. Hobart, Tasmania, Australia.

LEPAIS, O. \& BACLES, C. 2010. Comparison of random and SSR-enriched shotgun pyrosequencing for microsatellite discovery and single multiplex PCR optimisation in Acacia harpophylla F. Muell. Ex Benth. Molecular Ecology Resources 11:711-724.

LEVINSON, G. \& GUTMAN, G. A. 1987. Slipped-strand mispairing: a major mechanism for DNA sequence evolution. Molecular Biology and Evolution 4: 203-221.

LEWALLEN, E. A., ANDERSON, T. W. \& BOHONAK, A. J. 2007. Genetic structure of leopard shark (Triakis semifasciata) populations in California waters. Marine Biology 152: 599-609.

LI, Y. C., KOROL, A. B., FAHIMA, T., BEILES, A. \& NEVO, E. 2002. Microsatellites: genomic distribution, putative functions and mutational mechanisms: a review. Molecular Ecology 11: 2453-2465.

LUIKART, G. \& CORNUET, J. M. 1998. Empirical evaluation of a test for identifying recently bottlenecked populations from allele frequency data. Conservation Biology 12: 228-237.

LIBRADO, P \& ROZAS, J. 2009. DNAsp v5: A software for comprehensive analysis of DNA polymorphism data. Bioinformatics 25: 1451-1452.

MACDONALD, C. M. 1988. Genetic variation, breeding structure and taxonomic status of the gummy shark Mustelus antarcticus in Southern Australian waters. Australian Journal of Marine and Freshwater Research 39: 641-648.

MANIRE, C. A. \& GRUBER, S. H. 1990. Many sharks may be headed toward extinction. Conservation Biology 4:10-11.

MARTINEZ, J. L., MORAN, P., PEREZ, J., GAUDEMAR, B., BEALL, E. \& GARCIAVASZQUEZ, E. 2000. Multiple paternity increase effective size of southern Atlantic salmon populations. Molecular Ecology 9: 293-298. 
MARUSKA, K. P., COWIE, E. G. \& TRICAS, T, C. 1996. Periodic gonadal activity and protracted mating in Elasmobranch fishes. The Journal of Experimental Zoology 276: 219-232.

MCCAULEY, L., GOECKER, C., PARKER, P., RUDOLPH, T., GOETZ, F. \& GERLACH, G. 2004. Characterization and isolation of DNA microsatellite primers in the spiny dogfish (Squalus acanthias). Molecular Ecology Notes 4: 494-496.

MCLOUGHLIN, K. 2007. Shark Gillnet and Hook Sectors. In: LARCOMBER, J. \& MCLOUCHLIN, K. (eds.) Fishery Status Reports 2006: Status of Fish Stocks Managed by the Australian Government, Bureau of Rural Sciences, Canberra, Australia.

MEGLÉCZ, E., COSTEDOAT, C., DUBUT, V., GILlES, A., MALAUSA, T., PECH, N. \& MARTIN, J. F. 2010. QDD: a user-friendly program to select microsatellite markers and design primers from large sequencig projects. Bioinformatics 26: 403-404.

MENDONÇA, F. F., OLIVEIRA, C., FAZZANO, O, B, G. \& FORESTI, F. 2009. Populations analysis of the Brazilian sharpnose shark Rhizoprionodon lalandii (Chondrichthyes: Carcharhinidae) on the São Paulo coast, Southern Brazil: inferences from mtDNA sequences. Neotropical Ichthyology 7: 213-216.

MENDONÇA, F. F., USSAMI, L. H. F. HASHIMOTO, D. T. PEREIRA, L. H. G., PORTOFORESTI, F., OLIVEIRA, C., GADIG, O. B. F. \& FORESTI, F. 2012. Identification and characterization of polymorphic microsatellite loci in the blue shark Prionace glauca, and cross-amplification in other shark species. Journal of Fish Biology 80: 2643-2646.

MINISTRY OF PRIMARY INDUSTRIES (MPI). 2012. Report from the fisheries assessment plenary, May 2012: stock assessments and yield estimates. Compiled by the Fisheries Science Group, MPI, Wellington, New Zealand.

MOURA, T., SERRA-PEREIRA, B., GORDO, L. S. \& FIGUEIREDO, I. 2011. Sperm storage in males and females of the deepwater shark Portuguese dogfish with notes on oviductal gland microscopic organization. Journal of Zoology. 283: 210-219.

MUSICK, J. A., BURGESS, G., CAILliET, G, CAMHI, M. \& FORDHAM, S. 2000. Management of sharks and their relatives (Elasmobranchii). Fisheries 25:9-13.

MUSICK, J. A., HARBIN, M. M. \& COMPAGNO, L. J. V. 2004. Historical Zoogeography of the Selachii. In: CARRIER, J. C., MUSICK, J. A. \& HEITHAUS, M. R. (eds.) Biology of sharks and their relatives. Boca Raton, Florida, USA.

MUSICK, J. A. \& BONFIL, R. 2005. Management techniques for elasmobranch fisheries . FAO Fisheries Technical Paper. No. 474. Rome, Italy.

MURRAY, B. W., WANG, J. Y., YANG, S. C., STEVENS, J. D., FISK, A. \& SVAVARSSON, J. 2008. Mitochondrial cytochrome b variation in sleeper sharks (Squaliformes: Somniosidae). Marine Biology 153: 1015-1022.

MORRISSEY, J. F. \& GRUBER, S. H. 1993. Habitat selection by juvenile lemon sharks, Negaprion brevirostris. Environmental Biology of Fishes 38: 311-319. 
MYERS, R. A. \&WORM, B. 2003. Rapid worldwide depletion of predatory fish communities. Nature 423: 280-283.

NANCE, H. A., DALY-ENGEL, T. S. \& MARKO, P. B. 2009. New microsatellite loci for the endangered scalloped hammerhead shark, Sphyrna lewini. Molecular Ecology Resources 9: 955-7.

NEFF, B. D. \& PITCHER, T. E. 2002. Assessing the statistical power of genetic analyses to detect multiple mating in fishes. Journal of Fish Biology 61: 739-750.

NEI, M. T. M. \& CHAKRABORTY, R. 1975. The bottleneck effect and genetic variability in populations. Evolution 29: 1-10.

NEI, M., TAJIMA, F. \& TATENO, Y. 1983. Accuracy of estimated phylogenetic trees from molecular data II. Gene frequency data. Journal of Molecular Evolution 19: 153-170.

NIELSEN, E. E., HEMMER-HANSEN, J., LARSEN, P. F. \& BEKKEVOLD, D. 2009. Population genomics of marine fishes: identifying adaptative variation in space and time. Molecular Ecology 18: 3128-3150.

O’ BRIEN, S. M., GALLUCCI, V. F. \& HAUSER, L. 2013. Effects of species biology on the historical demography of sharks and their implicantions for likely consequences of contemporary climate change. Conservation Genetics 14: 125-144.

O'CONNELL, M. \& WRIGHT, J. M. Microsatellite DNA in fishes. Reviews in Fish Biology and Fisheries 7: 331-363.

OLSEN, A. M. 1953. Tagging of school, Galeorhinus galeus (Macleay)(Carcharhinidae), in south-eastern Australian waters. Australian Journal of Marine and Freshwater Research 4: 95-104.

OLSEN, A. M. 1954. The biology, migration and growth rate of the school shark Galeorhinus australis (Macleary) (Carcharhinidae) in southeastern Australian. Australian Journal of Marine and Freshwater Research 5: 353-410.

OLSEN, A. M. 1959. The status of the school shark fishery in South-Eastern Australian waters. Australian Journal of Marine and Freshwater Research 10: 150-176.

OLSEN, A. M. 1984. Species synopsis of school shark, Galeorhinus australis (Macleary, 1881). FAO Fisheries Synopsis No. 139. Rome, Italy.

OVENDEN, J., STREET, R. \& BRODERICK, D. 2006. New microsatellite loci for carcharhinids sharks (Carcharhinus tilstoni and C. sorrah) and their cross-amplification in other shark species. Molecular Ecology Notes 6: 415-418.

OVENDEN, J, R., KASHIWAGI, T., BRODERICK, D., GILES, J. \& SALINI, J. 2009. The extent of population genetic subdivision differs among four co-distributed shark species in the Indo-Australian archipelago. BMC Evolutionary Biology 9: 1-15.

OVENDEN, J. R., MORGAN, J., KASHIWAGI, T., BRODERICK, D. \& SALINI, J. 2010. Towards better management of Australia's shark fishery: genetic analyses reveal unexpected ratios of cryptic blacktip species Carcharhinus tilstoni and C. limbatus. Marine and Freshwater Research 61: 253-262. 
OVENDEN, J. R. 2013. Crinkles in connectivity: combining genetics and other types of biological data to estimate movement and interbreeding between populations. Marine and Freshwater Research 64: 201-207.

PALUMBI, S. R. 1994. Genetic divergence, reproductive isolation, and marine speciation. Annual Review of Ecology and Systematics 25: 547-572.

PARDINI, A., TARDINI, A. T., JONES, C. S., SCHOLL, M. C. \& NOBLE, L. R. 2000. Isolation and characterization of dinucleotide microsatellite loci in the great white shark, Carcharodon carcharias. Primer Notes 9: 1171-1193.

PARDINI, A. T., JONES, C. S., NOBLE, L. R., KREISER, B., MALCOLM, H., BRUCE, B. D., STEVENS, J. D., CLIFF, G., SCHOLL, M. C., FRANCIS, M., DUFFY, C. A. J. \& MARTIN, A. P. 2001. Sex-biased dispersal of great white shark. Nature 412: 139-140.

PARCHMAN, T., GEIST, K., GRAHNEN, J., BENKMAN, C. \& BUERKLE, C. A. 2010. Transcriptome sequencing in an ecologically important tree species: assembly, annotation, and marker discovery. BMC Genomics 9: 1171-1193.

PARSONS, G. R., HOFFMAYER, E. R., HENDON, J. M. \& BET-SAYAD, W. V. 2008. A review of shark reproductive ecology: life history and evolutionary implications. In: ROCHA, M. A., ARUKWE, A. \& KAPOOR, B. G. (eds) Fish Reproduction. Enfield, New Hampshire, USA.

PAUL, L. J. 1988. School shark. MAFFISH, N.Z. Ministry of Agriculture and Fisheries New Zealand Fisheries Assessment Research Document 88/27. Wellington, New Zealand.

PAUL, L. J. \& SAUNDERS, B. 2001. A description of the commercial fishery of school shark, Galeorhinus galeus, in New Zealand, 1945 to 1999. New Zealand Fisheries Assessment Report 2001/3. Wellington, New Zealand.

PEAKALL, R. \& SMOUSE, P. E. 2006. GENALEX 6: genetic analysis in Excel. Population genetic software for teaching and research. Molecular Ecology Notes 6: 288-295.

PERES, M. B. \& VOOREN, C. M. 1991. Sexual development reproductive cycle, and fecundity of the school shark Galeorhinus galeus off Southern Brazil. Fishery Bulletin 89: 655-667.

PERUMAL, R., NIMMAKAYALA, P., ERATTAIMUTHU, S. R., NO, E. G., REDDY, U. K., PROM, L. K., ODVODY, G. N., LUSTER, D. G. \& MAGILL, C. W. 2008. Simple sequence repeats markers useful for sorghum downy mildew (Peronosclerospora sorghi) and related species. BMC Genetics 9: 1-14.

PETIT, R. J, DEGUILlOUX, M. F. \& CHAT, J., GRIVET, D., GARNIER, P. G. \& VENDRAMIN, G. G. 2005. Standardizing for microsatellite length in comparisons of genetic diversity. Molecular Ecology 14: 885-890.

POSADA, D. 2008. jModelTest: phylogenetic model averaging. Molecular Biology and Evolution 25: 1253-1256.

PORTNOY, D. S., MCDOWELL, J. R., THOMPSON, K., MUSICK, J. A. \& GRAVES, J. E. 2006. Isolation and characterization of five dinucleotide microsatellite loci in the sandbar, Carcharhinus plumbeus. Molecular Ecology Notes 6: 431-433. 
PORTNOY, D. S. MCDOWELL, J. R., HEIST, E., MUSICK, J. A. \& GRAVES, J. E. 2010. World phylogeography and male-mediated gene flow in the sandar shark, Carcharhinus plumbeus. Molecular Ecology 19: 1994-2010.

PORTNOY, D. S. \& HEIST, E. J. 2012. Molecular markers: progress and prospects for understanding reproductive ecology in Elasmobranch. Journal of Fish Biology 80: 11201140.

PUNT, A. E. \& WALKER, T. I. 1998. Stock assessment and risk analysis for the school shark (Galeorhinus galeus) off southern Australia. Marine Freshwater Research 49: 719731.

PUNT, A. E., PRIBAC, F., WALKER, T. I., TAYLOR, B. L., PRINCE, J. D. 2000. Stock assessment of school shark, Galeorhinus galeus, based on a spatially explicit population dynamics model. Marine Freshwater Research 51: 205-220.

PUNT, A. E., PRIBAC, F., TAYLOR, B. L. \& WALKER, T. I. 2005. Harvest strategy evaluation for school shark and gummy shark. Journal of Northwest Atlantic Fishery Science 35: 387-406.

PRATT, H. L. 1993. The storage of spermatozoa in the oviducal glands of western North Atlantic sharks. Environmental Biology of Fishes 38: 139-149.

PRATT. H. L. \& Carrier, J. C. 2001 A review of elasmobranch reproductive behavior with a case study on the nurse shark, Ginglymostoma cirratum. Environmental Biology of Fishes 60: $157-188$.

PRITCHARD, J. K., STEPHENS, M. \& DONNELLY, P. 2000. Inference of population structure from multilocus genotype data. Genetics 155: 945-959.

PYBUS, O. G., RAMBAUT, A. \& HARVEY, P. H. 2000. An integrated framework for the inference of viral population history from reconstructed genealogies. Genetics 155: 14291437.

PYRI, S., LUIKART, G. \& CORNUET, J. M. 1999. BOTTLENECK: A computer program for detecting recent reductions in the effective population size using allele frequency data. The Journal of Heredity 90: 502-503.

RAMBAUT, A. \& DRUMMOND, A. J. 2009. Tracer v1.5.0. Available from http://beast.bio.ed.ac.uk/Tracer.

RAMOS-ONSINS, S. E. \& ROZAS, J. 2002. Statistical properties of new neutrality tests against population growth. Molecular Biology and Evolution 13: 235-248.

RAMÍREZ-MARCÍAS, D., VÁSQUEZ-JUÁREZ, R., GALVÁN-MAGAÑA， F. \& MUNGUÍA-VEGA, A. 2007. Variations of the mitochondrial control region sequence in whale sharks (Rhincodon typus) from the Gulf of California, Mexico. Fisheries Research 84: 87-95.

RAMÍREZ-MARCÍAS, D., SHAW， K., WARD， R., GALVÁN-MAGAÑA，F. \& VÁSQUEZ-JUÁREZ, R. 2009. Isolation and characterization of microsatellite loci in the whale shark (Rhincodon typus). Molecular Genetic Resources 9: 798-800. 
RAYMOND, M. \& ROUSSET, F. 1995. GENEPOP (version 1.2): population genetics software for exact tests and ecumenicism. Journal of Heredity 86: 248-249.

REID, D. D. \& KROUGH, M. 1992. Assessment of catches from protective shark meshing off New South Wales beaches between 1950 and 1990. Australian Journal of Marine and Freshwater Research 43: 283-296.

RICE, W. R. 1989. Analyzing tables of statistical tests. Evolution 43: 223-225.

RICHARD, V. P., HENNING, M., WITZELL, W. \& SHIVJI, M. S. 2009. Species delineation and evolutionary history of the globally distributed spotted eagle ray (Aetobatus narinari).Journal of Heredity 100: 273-283.

RIOUX, P. S. PopGenKit: useful functions for (batch) file conversion and data resampling in microsatellite datasets. 2011. $\mathrm{R}$ package, version 1.0. Available from http://cran.rproject.org/web/packages/PopGenKit/index.html

ROGER, A. R. \& HARPENDING, H. 1992. Population growth makes waves in the distribution of pairwise genetic differences. Molecular Biology and Evolution 9: 552569.

ROSEL, P. E. \& BLOCK, B. A. 1996. Mitochondrial control region variability and global population structure in the swordfish, Xiphias gladius. Marine Biology 125: 11-22.

ROWE, S. \& HUTCHINGS, J. A. 2003. Mating systems and the conservation of commercially exploited marine fish. Trends in Ecology and Evolution 18: 567-572.

ROZEN, S. \& SKALETSKY, H, J. 2000. Primer3 on the WWW for general users and for biologist programmers. In: KRAWETZ, S. \& MISENER, S. (eds.) Bioinformatics Methods and Protocols: Methods in Molecular Biology. Humana Press, Totowa, New Jersey, USA.

RYMAN, N. \& PALM, S. 2006. POWSIM: a computer program for assessing statistical power when testing for genetic differentiation. Molecular Ecology 6: 600-602.

SALZBURGER, W., EWING, G. B. \& HAESELER, A. 2011. The performance of phylogenetic algorithms in estimating haplotype genealogies with migration. Molecular Ecology 20: 1952-1963.

SAMBROOK, J., FRITSCH, E. \& MANIATIS, T. 1989. Molecular clonning: A laboratory manual. Cold Spring Harbor Laboratory, New York.

SAVILLE, K. J., LINDLEY, A. M., MARIES, E. G., CARRIER, J. C. \& PRATT, H. L. 2002. Multiple paternity in the nurse shark, Ginglymostoma cirratum. Environmental Biology of Fishes 63: 347-351.

SCHLÖTTERER, C. \& TAUTZ, D. 1992. Slippage synthesis of simple sequence DNA. Nucleic Acids Resource 20: 211-215.

SCHLUESSEL, V., BRODERICK, D., COLLIN, S. P. \& OVENDEN, J. R. 2010. Evidence for extensive population structure in the white spotted eagle ray within the Indo-Pacific inferred from mitochondrial gene sequences. Journal of Zoology (London) 281, 46-55. 
SCHMIDT, J. V., SCHMIDT, C. L., OZER, F., ERNST, R. E. FELDHEIM, K. A., ASHLEY, M. V. \& LEVINE, M. 2009. Low genetic differentiation across three major ocean populations of the whale shark, Rhincodon typus. PLOS ONE 4: 1-9.

SCHMIDT, J. V., CHEN, C. C., SHEIKH, S. I., MEEKAN, M. G., NORMAN, B. M.\& JOUNG, S. J. 2010. Paternity analysis in a litter of whale shark embryos. Endangered Species Research 12: 117-124.

SCHREY, A. W. \& HEIST, E. J. 2002 Microsatellite markers for the shortfin mako and cross-species amplification in lamniformes. Conservation Genetics 3: 459-461.

SCHREY, A. W. \& HEIST, E. J. 2003 Microsatellite analysis of population structure in the shortfin mako (Isurus oxyrinchus). Canadian Journal of Fisheries and Aquatic Sciences 3: 459-461.

SCHUELKE, M. 2000. An economic method for the fluorescent labelling of PCR fragments. Nature Biotechnology 18: 233-234.

SCHUG, M. D., WETTERSTRAND, K. A., GAUDETTE, M. S., LIM, R. H., HUTTER, C. M. \& AQUADRO, C. F. 1998. The distribution and frequency at microsatellite loci in Drosophila melanogaster. Molecular Ecology 7: 57-70.

SCHULTZ, J. K., FELDHEIM, K. A., GRUBER, S. H. ASHLEY, M. V., McGOVERN, T. M. \& BOWEN, B. W. 2008. Global phylogeography and seascape genetics of the lemon sharks (genus Negaprion). Molecular Ecology 17: 5336-5348.

SELKOE, K. A., HENZLER, C. M. \& GAINES, S. D. 2008. Seascape genetics and the spatial ecology of marine populations. Fish and Fisheries 4: 363-377.

SELLAS, A. B., BASSOS-HULL, K., HUETER, R. E. \& FELDHEIM, K. A. 2011. Isolation and characterization of polymorphic microsatellite markers from the spotted eagle ray (Aetobatus narinari). Conservation Genetic Resources 3: 609-611.

SHUELKE, M. 2000. An economic method for the fluorescent labelling of PCR fragments. SAMBROOK, J., FRITSCH, E. F. \& MANIATIS, T. 1989. Molecular Cloning: A laboratory manual $2^{\text {nd }}$ Edition. CSH Laboratory Press, Cold Spring Harbor, New York.

SLATKIN, M. \& EXCOFFIER, L. 1996. Testing for linkage disequilibrium in genotypic data using the EM algorithm. Heredity 76: 377-383.

SMALE, M. J. \& COMPAGNO, L. J. V. 1997. Life history and diet of two southern African smoothhounds sharks, Mustelus mustelus (Linnaeus, 1758) and Mustelus palumbes Smith, 1957 (Pisces: Triakidae). South African Journal of Marine Science 18: 229-248.

SMITH, S. E., AU, D. W. \& SHOW, C. 1998. Intrinsic rebound potential of 26 species of Pacific sharks. Marine Freshwater Research 49: 663-678.

SMITH, P. J. 1986. Low genetic variation in sharks (Chondrichthyes). Copeia1986: 202-207.

SMITH, P. J. \& BENSON, P. G. 2001. Biochemical identification of shark fins and fillets from the coastal fisheries in New Zealand. Fishery Bulletin 99: 351-355.

SUGG, D. W. \& CHESSER, R. K. 1994. Effective population sizes with multiple paternity. Genetics 137: 1147-1155. 
STANLEY, C. A. 1988. Tagging experiments on school shark (Galeorhinus australis) and gummy shark (Mustelus antarcticus): recapture data for South-eastern Australian releases, 1942 to 1956. CSIRO Marine Laboratories Report No. 192, Hobart, Australia.

STEVENS, J. D. \& WEST, G. J. 1997. Investigation of school and gummy shark nursery areas in South Eastern Australia. FRDC Project 93/061, Hobart, Australia.

STEVENS, J. D., BONFIL, R., DULVY, N. K., WALKER, P. A. 2000. The effects of fishing on sharks, rays, and chimeras (chondrichthyans), and the implications for marine ecosystems. ICES Journal of Marine Science 57: 476-494.

STEVENS, J. 2005. Tope or school shark Galeorhinus galeus (Linneaus, 1758).In: FOWLER, S. L., CAVANAGH, R. D., CAMHI, M., BURGESS, G. H., CAILlIET, G. M., FORDHAM, S. V., SIMPFENDORFER, C. A. \& MUSICK, J. A. (eds.) Sharks, Rays and Chimaeras: The Status of theChondrichthyan Fishes, Switzerland and Cambridge, $\mathrm{UK}$.

STORRIE, M. T., WALKER, T. I., LAURENSON, L. \& HAMLETT, W. C. 2008. Microscopic organization of the sperm storage tubules in the oviductal gland of the female gummy shark (Mustelus antarcticus), with observations on sperm distribution and storage. Journal of Morphology 269: 1308-1324.

STOW, A., ZENGER, K., BRISCOE, D., GILlinGS, M., PEDDEMORS, V., OTWAY, N. \& HARCOURT, R. 2006. Isolation and genetic diversity of endangered grey nurse shark (Carcharias taurus) populations. Biology Letters 2: 308-311.

STRAUBE, N., KRIWET, J. \& SCHLIEWEN, U. K. 2011. Cryptic diversity and species assignment of large lantern sharks of the Etmopterus spinax clade from the Southern Hemisphere (Squaliformes, Etmopteridae). Zoologica Scripta 40: 61-75.

SWOFFORD, D. L. 2000. PAUP*. Phylogenetic Analysis Using Parsimony (*and other Methods) Version 4. Sinauer, Sunderland, Massachusetts, USA. Nature Biotechnology 18: 233-234.

TAGUCHI, M., KITAMURA, T., SHIGENOBU, Y., OHKUBO, M., YAMAGIMOTO, T., SUGAYA, T., NAKAMURA, Y., SAITOH, K. \& YOKAWA, K. 2013. Development of 15 polymorphic microsatellite markers for the shortfin mako, Isurus oxyrinchus, and cross-species amplification in lamniforme sharks. Conservation Genetics Resources 14: 223-229.

TAJIMA, F. 1989. Statistical-method for testing the neutral mutation hypothesis by DNA polymorphism. Genetics 123: 585-595.

TESTERMAN, C., PRODOHL, P. \& SHIVJI, M. 2008. Global phylogeography of the great (Sphyrna mokarran) and smooth (Sphyrna zygaena) hammerhead sharks. Paper presented in the American Elasmobranch Society (AES), Systematics \& Biogeography II.

THREATENED SPECIES SCIENTIFIC COMMITEE (TSSC). 2009. Addendum to the School Shark Rebuilding Strategy 2008. Department of the Environment, Water, Heritage and the Arts. Available from http://www.environment.gov.au/biodiversity/threatened/species/pubs/68453-addenumrebuilding-strategy.pdf. 
TILLET, B. J., MEEKAN, M. J., FIELD, I., THORBURN, D. \& OVENDEN, J. (in review). Evidence for reproductive philopatry in the bull shark, Carcharhinus leucas in northern Australia. Journal of Fish Biology.

VAN OOSTERHOUT C., HUTCHINSON, W., WILLS, D. \& SHIPLEY, P. 2004.

Microchecker: software for identifying and correcting genotyping errors in microsatellite data. Molecular Ecology Notes 4: 535-538.

VARELA, A. I., RITCHIE, P. A. \& SMITH, P. J. 2012. Low levels of global genetic differentiation and population expansion in the deep-sea teleost Hoplostethus atlanticus revealed by mitochondrial DNA sequences. Marine Biology 140: 83-90.

VARELA, A. 2013. Population genetics of the teleost orange roughy, Hoplostethus atlanticus, and insights into their visual adaptations to the deep-sea environment. Ph.D. thesis, Victoria University of Wellington, Wellington. New Zealand.

VERÍSSIMO, A., MCDOWELL, J. R. \& GRAVES, J. E. 2010. Global population structure of the spiny dogfish Squalus acanthias, a temperate shark with an antitropical distribution. Molecular Ecology 19: 1651-1662.

VERÍSSIMO, A., MCDOWELL, J. R. \& GRAVES, J. E. 2010. Population structure of a deep-water squaloid shark, the Portuguese dogfish (Centroscymnus coelolepis). ICES Journal of Marine Science 68: 555-563.

VERÍSSIMO, A., MOURA, T., MCDOWELL, J. R., GRAVES, J. E., GORDO, L. \& HOELZEL, R. 2011. Isolation and characterization odf ten nuclear microsatellite loci for the portuguese dogfish Centroscymnus coelolepis. Conservation Genetic Resources 3: 299-301.

WALKER, T. I. 1989. Methods of tagging adopted in the southern shark fishery. In: HANCOCK, D. A (eds.) Tagging - solution or problem? Australian Government Publishing Service. Canberra, Australia.

WALKER, T. I., BROWN, L. P., BRIDGE, N. F. 1997. Southern Shark Tagging Project. Final Report to Fisheries Research and Development Corporation. FRDC Project 93/066. Queenscliff, Australia.

WALKER, T. I. 1999. Galeorhinus galeus fisheries of the world. In: SHOTTON, R (eds.) Case studies of management of Elasmobranch fisheries. FAO Fisheries Technical Paper No. 387/2. Rome, Italy.

WALKER, T. I. 2000. Modelling school shark (Galeorhinus galeus) movement in the southern shark fishery. In: HANCOCK, D. A., SMITH, D. C. \& KOEHN, J. D. (eds.) Movement and migration. Australian society for fish biology workshop proceeding, Victoria, Australia.

WALKER, T. I. 2005. Reproductive in Fisheries Science. In: HAMLETT, W (eds.) Reproductive Biology and Phylogeny of Chondrichthyes, Sharks, Batoids and Chimaeras. Science Publishers, Inc., Enfield, USA.

WALKER, T. I., CAVANAGH, R. D. \& STEVENS, J. D., CARLiSle, A. B., CHIARAMONTE, G., DOMINGO, A., EBERT, D. A., MANCUSI, C. M., MASSA, A., 
MCCORD, M., MOREY, G., PAUL, L. J., SERENA, F. \& VOOREN, C.M. 2006. Galeorhinus galeus. In: IUCN 2007. IUCN Red List of Threatened Species.

WALKER, T. I. 2007. Spatial and temporal variation in the reproductive biology of gummy shark Mustelus antarcticus (Chondrichthyes: Triakidae) harvested off southern Australia. Marine and Freshwater Research 58: 67-97.

WALKER, T. I., TAYLOR, B. L., BROWN, L. P. \& PUNT, A. E. 2008. Embracing Movement and Stock Structure for Assessment of Galeorhinus galeusHarvested off Southern Australia. In: CAMHI, M. D., PIKITCH, E. K. \& BABCOCK, E. A (eds.) Shark of the Open Ocean Biology, Fisheriesand Conservation. Blackwell Publishing, Victoria, Australia.

WANG, Z. WEBER, J. L., ZHONG, G., TANKSLEY, S. D. 1994. Survey of plant short tandem DNA repeats. Theoretical and Applied Genetics 88: 1-6.

WARD, R. D. \& GARDNER, M. G. 1997. Stock structure and species identification of school and gummy sharks in Australasian waters. Project FRRF 93/11 and FRDC 93/64, Hobart, Australia.

WARD, R. D. \& GARDNER, M. G. 1998. Population structure of the Australian gummy shark (Mustelus antarcticus Günther) inferred from allozymes, mitochondrial DNA, and vertebrae counts. Marine and Freshwater Research 49: 733-745.

WARD, R. D. 2000. Genetics in fisheries management. Hidrobiologia 420: 191-201.

WARD, R. D. \& ELLIOTT, N. G. 2001. Genetic population structure of species in the south east fishery of Australia. Marine Freshwater Research 52: 563-573.

WARD, R. D., HOLMES, B. H., ZEMLAK, T. S. \& SMITH, P. J. 2007. DNA barcoding discriminates spurdogs of the genus Squalus. In: LAST, P. R., WHITE, W. T. \& POGONOSKI, J. J. (eds) Descriptions of New Dogfishes of the genus Squalus (Squaloidea: Squalidae), CSIRO Marine and Atmospheric Research, Tasmania, Australia.

WARD, R. D., HOLMES, B. H., WHITE, W. T. \& LAST, P. R. 2008. DNA barcoding Australasian chondricthyans: results and potential uses in conservation. Marine and Freshwater Reasearch 59: 57-71.

WAPLES, R. S. 1987. A mnitispecies approach to the analysis of gene flow in marine shore fishes. Evolution 41, 385-400

WAPLES, R. S. 1998. Separating the wheat from the chaff: patterns of genetic differentiation in high gene flow species. Journal of heredity 89: 438-450.

WAPLES, R. S., PUNT, A. E. \& COPE, J. M. 2008. Integrating genetic data into management of marineresources: how ca we do it better? Fish and Fisheries 9: 423-449.

WEARMOUNTH, V. J., SOUTHALL, E. J., MORRITT, D., THOMPSON, R. C., CUTHILL, I. C., PARTRIDGE, J. C. \& SIMS, D. W. 2012. Year-round sexual harassment as a behavioural meaditor of vertebrate population dynamics. Ecological Monographs 83: 351-366. 
WEBER, J. L. 1990. Informativeness of human (dC-dA)n (dG-dT)n polymorphisms. Genomics: 7 524-530.

WEBER, J. L. \& WONG, C. 1993. Mutation of Human Short Tandem Repeats. Human. Molecular Genetics 2: 1123-1128.

WEIR, B. S \& COCKERHAM, C. C. 1984. Estimating F-statistics for the analysis of population structure. Evolution 38: 1358-1370.

WHITNEY, N. M., PRATT, H. L. \& CARRIER, J. C. 2004. Group courtship, mating behaviour and siphon sac function in the whitetip reef shark, Triaenodon obesus. Animal Behaviour 68: 1435-1442.

WOURMS, J. P. 1977. Reproduction and development in condrichthyan fishes. American Zoology 17: 379-410.

WOURMS, J. P. \& DEMSKI, L. S. 1993. The reproduction and development of sharks, skates, rays and ratfishes: introduction, history, overview, and future prospects. Environmental Biology of Fishes 38: 7-21.

WEST, G. J. \& STEVENS, J. D. 2001. Archival tagging of school shark,Galeorhinus galeus, in Australia: initial results. Environmental Biology of Fishes 60: 283-298.

YANO, K., SATO, F. \& TAKAHASHI, T. 1999. Observations of mating behaviour of the manta ray, Manta birostris, at the Ogasawara Islands, Japan. Ichthyological Research 46: 289-296

YAGISHITA, N. \& YAMAGUCHI, A. 2009. Isolation and characterization of eight microsatellite loci from the longheaded eagle ray, Aetobatus flagellum (Elasmobranchii, Myliobatidae). Molecular Genetic Resources 9: 1034-1036.

YAMAGUCHI, A., TANIUCHI, T. \& SHIMIZU, M. 1997. Reproductive biology of the starspotted dogfish Mustelus manazo from Tokyo Bay, Japan. Fisheries Science 63: 918922.

ZEH, J. A. \& ZEH, D. W. 2003. Toward a new sexual selection paradigm: polyandry, conflict and incompatibility. Ethology 109: 929-950. 